\title{
A dynamic model of the onset of clinical psychosis from an epidemiological perspective
}

Citation for published version (APA):

Domínguez Barrera, M. D. G. (2009). A dynamic model of the onset of clinical psychosis from an epidemiological perspective. [Doctoral Thesis, Maastricht University]. Datawyse / Universitaire Pers Maastricht. https://doi.org/10.26481/dis.20091217md

Document status and date:

Published: 01/01/2009

DOI:

10.26481/dis.20091217md

Document Version:

Publisher's PDF, also known as Version of record

\section{Please check the document version of this publication:}

- A submitted manuscript is the version of the article upon submission and before peer-review. There can be important differences between the submitted version and the official published version of record.

People interested in the research are advised to contact the author for the final version of the publication, or visit the DOI to the publisher's website.

- The final author version and the galley proof are versions of the publication after peer review.

- The final published version features the final layout of the paper including the volume, issue and page numbers.

Link to publication

\footnotetext{
General rights rights.

- You may freely distribute the URL identifying the publication in the public portal. please follow below link for the End User Agreement:

www.umlib.nl/taverne-license

Take down policy

If you believe that this document breaches copyright please contact us at:

repository@maastrichtuniversity.nl

providing details and we will investigate your claim.
}

Copyright and moral rights for the publications made accessible in the public portal are retained by the authors and/or other copyright owners and it is a condition of accessing publications that users recognise and abide by the legal requirements associated with these

- Users may download and print one copy of any publication from the public portal for the purpose of private study or research.

- You may not further distribute the material or use it for any profit-making activity or commercial gain

If the publication is distributed under the terms of Article $25 \mathrm{fa}$ of the Dutch Copyright Act, indicated by the "Taverne" license above, 
A Dynamic Model of the Onset of Clinical Psychosis from an Epidemiological Perspective 


\section{ISBN 9789052788968}

(C) Copyright María de Gracia Domínguez Barrera, Maastricht 2009

Printed by Datawyse / Universitaire Pers Maastricht

Cover illustration: Multitud, by Luis Gutiérrez Dubón. http://www.dubon.es 


\title{
A Dynamic Model of the Onset of Clinical Psychosis from an Epidemiological Perspective
}

\author{
PROEFSCHRIFT \\ ter verkrijging van de graad van doctor aan de Universiteit Maastricht, \\ op gezag van de Rector Magnificus, Prof. mr. G.P.M.F. Mols, \\ volgens het besluit van het College van Decanen, \\ in het openbaar te verdedigen \\ op donderdag 17 december 2009 om 16:00 uur
}

door

María de Gracia Domínguez Barrera geboren op 9 november 1974 te Pamplona

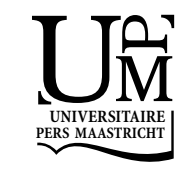




\section{Promotor}

Prof. dr. J. van Os

\section{Copromotores}

Dr. L. Krabbendam (Vrij Universiteit Amsterdam)

Dr. M. Wichers

\section{Beoordelingscommissie}

Prof. dr. M. de Vries (voorzitter)

Dr. P. Delespaul

Prof. dr. D. Linszen (Universiteit van Amsterdam)

Dr. I. Myin-Germeys

Prof. dr. D. Wiersma (Universitair Medisch Centrum Groningen)

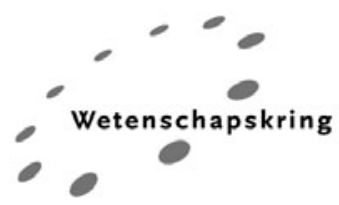

South Limburg Mental Health Research and Teaching Network PhD Series 
When you set out on your journey to Ithaca, hope that the road is long, full of adventure, full of discovery. Laistrygonians, Cyclops, angry Poseidon -- do not fear them: You will never find such as these on your way, as long as you keep your thoughts raised high, as long as a fine emotion touches your spirit and your body. Laistrygonians, Cyclops, the fierce Poseidon -- you will never encounter them, unless you bring them alone inside your soul, unless your soul sets them up in front of you.

Hope that the road is long. That the summer mornings are many, when, with such pleasure, with such joy you will enter harbours you are seeing for the first time; may you stop at Phoenician markets, and purchase fine merchandise, mother-of-pearl and coral, amber and ebony, sensual perfumes of all kinds, as many sensual perfumes as you can; and may you visit many Egyptian cities, to learn and learn from their scholars.

Always keep Ithaca in your mind. Arriving there is what you are destined for. But do not hurry the journey at all.

Better if it lasts for years; and to anchor at the island when you are old, wealthy with all you have gained on the way, not expecting Ithaca to make you rich.

Ithaca has given you the marvellous journey. Without her you would have never set out. She has nothing left to give you now.

And if you find her poor, Ithaca won't have fooled you.

Wise as you will have become, so full of experience, you will have understood by then what these Ithacas mean. 


\section{Paranimfen}

Nicole Geschwind

Anne Fett 
Introduction

\section{CHAPTER 2}

Are Psychotic Psychopathology and Neurocognition Orthogonal? A

Systematic Review of their Associations

\section{CHAPTER 3}

Evidence that Onset of Clinical Psychosis is an Outcome of Progressively More Persistent Subclinical Psychotic Experiences: An 8-Year Cohort Study

\section{CHAPTER 4}

Affective Dysregulation and Reality Distortion: A 10-Year Prospective Study of their Association and Clinical Relevance

\section{CHAPTER 5}

Onset of Clinical Psychosis: Negative/Disorganized Symptoms Increasing Risk for Positive Symptoms Increasing Risk for Impairment? A 10-Year Study

\section{CHAPTER 6}

Discussion

SUMMARY 



\section{CHAPTER 1}

INTRODUCTION 


\section{INTRODUCTION: CONTENTS}

\section{Psychosis in Clinical Settings}

Schizophrenia, the Paradigm of Psychotic Disorders

The Group of Psychotic Disorders

Phenomenological Heterogeneity of Psychotic Disorders

Associations among Psychosis Dimensions: A Narrative Review

Associations between Psychosis Dimensions and Intermediary Phenotypes:

Neurocognition

A Consilience Model for Clinical Psychosis

\section{Psychosis from an Epidemiological Perspective}

Mapping Epidemiological Research on Psychosis in the General Population Population-based Work on Psychotic-Like Experiences

Psychometric Assessment with Self-report Instruments

Clinical relevance versus Categorical Diagnosis

An Epidemiological Prediction of the Onset of Clinical Psychosis 
The work presented in this thesis starts from a theoretical model of the development of psychosis, which posits interactive mechanisms by which the individual's biopsychosocial environments contribute to the development of major psychiatric disorders. This thesis specifically examines how the psychosis phenotype exists in nature and the dynamic process driving psychosis expression from an epidemiological perspective.

Firstly, this introductory chapter describes the concept of psychosis as a clinical construct, providing a comprehensive literature review of clinical psychosis, the symptomatic presentation which has replicated associations with psychometric expression of psychosis.

In the second part, this introduction will turn to the epidemiological perspective, presenting the development of epidemiological research in recent years and postulating the hypothesis of the present thesis.

\section{Psychosis in Clinical Settings}

\section{Schizophrenia, the Paradigm of Psychotic Disorders}

Schizophrenia is an enigmatic mental disorder, which ranks among the world's top ten causes of long-term disability (Murray \& Lopez, 1996), with a high economic cost extending beyond the health care system to public sector bodies, lost income due to unemployment, leisure (Van Os \& Kapur, 2009). The concept of schizophrenia is based on the work of Kraepelin, who emphasised the long-term deteriorating course of the syndrome that was later characterized by the coexistence of (i) core cognitive impairment (Kraepelin, 1919), (ii) the "Bleulerian blend" of negative symptoms (Bleuler, 1950), such as apathy, social withdrawal, difficulties in thinking straight (loosening of associations), incongruous or flattened affect, loss of goal-directed behaviour or ambivalence due to conflicting impulses, and retreat into an inner world (autism); and (iii) the cluster of "Schneiderian First Rank Symptoms" (Schneider, 1959) including auditory hallucinations, delusional ideas of persecution, conspiracy, thought interference and passivity phenomena. According to DSM-IV (American Psychiatric Association, 1994) and ICD-10 (World Health Organization, 1990b), a diagnosis of schizophrenia is established when this syndrome interferes with social and occupational functioning, in terms of impaired functioning in work, school, parenting, self-care, independent living, interpersonal relationships and leisure time, so that individuals result in need for care and, consequently, reach patient status. 


\section{The Group of Psychotic Disorders}

Not only 'schizophrenia' but another five mental disorders are identified in the DSM-IV group of non-affective psychotic disorders, named 'schizoaffective disorder', 'schizophreniform disorder', 'delusional disorder', 'brief psychotic disorder' and 'psychotic disorder not otherwise specified'. When affective and substance-induced psychoses are considered, four more diagnostic categories are described: 'bipolar disorder' with psychotic features, 'major depressive disorder' with psychotic features, 'alcohol-induced' and other 'substance-induced psychotic disorders'. Together with 'psychotic disorders due to other medical conditions', these 10 psychotic categories share a phenomenological manifestation: the presence of positive psychotic symptoms. Delusions and hallucinations are thus not exclusive to schizophrenia and psychotic disorders occur across a range of diagnostic categories. Differential diagnosis between these different categories of psychotic disorder is based on duration, symptom patterns, dysfunction, associated substance use, "bizarreness" of delusions and presence of depression or mania. However, the resulting psychotic diagnostic categories show overlap in genetic liability (Kendler \& Diehl, 1993) and with bipolar disorder (Craddock \& Owen, 2007), suggesting a broad underlying liability that expresses across the different categories.

Generations of psychiatrists have been taught that schizophrenia affects $1 \%$ of the adult population in the world (American Psychiatric Association, 1994). A systematic review of studies considering the diagnostic category of schizophrenia in isolation revealed a lifetime prevalence and incidence of $0.30 \%-0.66 \%$ and $10.2-$ 22.0 per 100,000 person-years, respectively (Easton \& Chen, 2006; McGrath, Saha, Chant, \& Welham, 2008). Rates vary 3-fold depending on the diagnostic definition of schizophrenia that is used: a 'narrow' definition including patients with longer illness duration, younger age and more negative symptoms has lower rates than a 'broad' definition with less specific criteria (Castle, Wessely, \& Murray, 1993). However, when a broad definition of psychotic disorder was examined, including diagnostic categories such as 'delusional disorder', 'brief psychotic disorder' and 'psychotic disorder not otherwise specified', a lifetime prevalence rate of psychotic categories of $2.3 \%$ was obtained (Perala et al., 2007), rising to 3.5\% if bipolar disorder and substance-induced psychotic disorder were included.

Schizophrenia is a polythetic disease showing marked clinical heterogeneity in symptoms, determinants, course, and prognosis across individuals (van Os \& Kapur, 2009). The cause of psychotic disorders is unknown, but evidence suggests that multiple susceptibility genes (Harrison \& Owen, 2003), each with small effect, and acting in concert with (van Os \& Marcelis, 1998) environmental influences e.g. obstetric complications (M. Cannon, Jones, \& Murray, 2002), social-environmental factors, such as urbanicity (Krabbendam \& van Os, 2005), migration (Cantor-Graae 
\& Selten, 2005), cannabis use (D’Souza et al., 2004), social disadvantage (Morgan et al., 2008) and trauma (Morrison, Frame, \& Larkin, 2003) contribute. Definitions of schizophrenia with emphasis on negative symptoms and persistence of symptoms (associated with poorer outcome) have higher incidence rates for men (Roy, Maziade, Labbe, \& Merette, 2001), whereas for broader definitions which include affective symptoms and brief presentations (associated with better outcome, including fewer hospital admissions and better social functioning) the gender ratio is closer to unity (Castle et al., 1993). Similarly, the course and outcome of schizophrenia is characterized by largely unexplained (van Os \& Allardyce, 2009) heterogeneity rather than uniformly poor outcome, with a systematic review of follow-up studies showing poor outcome in less than $50 \%$ of patients and good outcome in less than $50 \%$ of patients in one year time (Menezes, Arenovich, \& Zipursky, 2006).

\section{Phenomenological Heterogeneity of Psychotic Disorders}

While psychotic disorders have manifestly similar clusters/dimensions of positive psychotic symptoms, the psychotic diagnostic categories encapsulate patients with markedly different clinical features. In fact, evidence has shown that quantitatively different psychopathology define the diagnostic phenotype in psychotic disorder (Dikeos et al., 2006; Grube, Bilder, \& Goldman, 1998; McGorry, Bell, Dudgeon, \& Jackson, 1998). Researchers have tried to reduce heterogeneity by defining subtypes of the disorder. e.g. 'positive' and 'negative' symptom clusters. Clinical scales such as the Brief Psychiatric Rating Scale (Overall \& Gorham, 1962), the Scale for the Assessment of Negative Symptoms (N.C. Andreasen, 1981) and the Scale for the Assessment of Positive Symptoms (N.C. Andreasen, 1984), the Positive and Negative Syndrome Scale for schizophrenia (Kay, Fiszbein, \& Opler, 1987) as well as the Signs and Symptoms of Psychotic Illness (Liddle, 1992) were developed for the quantitative measurement of such symptoms domains. This provides a two syndromic framework (typologies) (N. C. Andreasen \& Olsen, 1982; Carpenter, Heinrichs, \& Wagman, 1988; Crow, 1980; Kay, 1990), which have been integrated into classification systems proposing subtypes within the diagnostic categories (Allardyce, Gaebel, Zielasek, \& van Os, 2007).

Over the last three decades the development of intense computational procedures, such as exploratory factor analysis has allowed studies to identify psychopathological dimensions, which are groups of symptoms which co-occur more often than would be expected by chance alone. An individual can then be defined by how high or low they score on the different continuously scored latent dimensions, which may co-exist (Allardyce, Suppes, \& Van Os, 2007). A metaanalysis of 10 studies of clinical schizophrenia (published prior to 1994) based on exploratory factor analyses of symptom ratings confirmed a three factor solution: 1) 
the 'positive' or reality distortion, 2) 'negative' or psychomotor poverty, and 3) the 'disorganized' dimension (Grube et al., 1998). When measures of affective symptomatology are considered, a five factor solution is found adding the depressive and the manic or excitement dimensions (Dikeos et al., 2006; Good et al., 2004; Lindenmayer, Grochowski, \& Hyman, 1995; van Os, Gilvarry et al., 1999). The 5-dimensional model is found across the full spectrum of functional psychotic disorders including affective disorders (Lindenmayer et al., 2004; van Os, Gilvarry et al., 1999) and has reasonable internal validity (Dikeos et al., 2006; Lindenmayer et al., 2004). Models which split psychotic and affective symptoms on separate dimensions fit the data best (Dikeos et al., 2006; Serretti \& Olgiati, 2004), though there may be conflation of the disorganised and negative dimensions, in first onset samples (McGorry et al., 1998).

The five dimensional model (Figure 1) describes (i) the 'positive' dimension including symptoms of delusions, ideas of reference, unusual thought content, hallucinations, grandiosity and suspiciousness/persecution; (ii) the 'negative' dimension including those of alogia, affective flattening, avolition, apathy, anhedonia, asociality, social withdrawal, stereotyped thinking and motor retardation; (iii) the 'disorganized' dimension distinguishing conceptual disorganization, positive formal thought disorder, difficulty in abstract thinking, derailment, tangentiallity, incoherence, illogicality, circumstantiality, associative loosening, inattention/distractibility, disorientation, inappropriate affect, bizarre behavior, mannerisms and posturing; (iv) the 'depressive' dimension consisting of observed depression, hopelessness, self-depreciation, feelings of guilt, guilty ideas of reference, early wakening, suicidal ideation, anxiety and active social avoidance; and ( $\mathrm{v}$ ) the 'manic/excitement' dimension comprising pressured speech, excessive activity, thoughts racing, elevated mood, increased sociability, reduced need for sleep, reckless activity, irritable mood, increased self-esteem and grandiose delusions.

Figure 1. The Five Dimensional Model of the Psychosis Phenotype

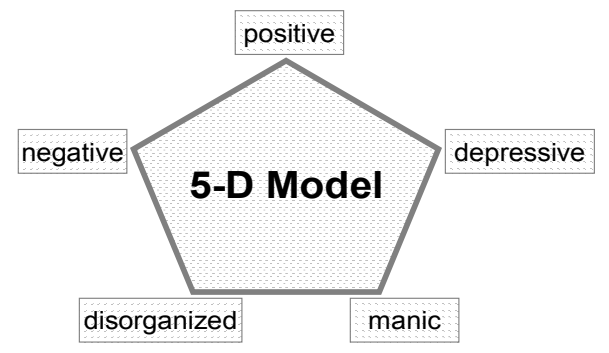

Note: 5-D: 5 Dimensional; Positive: Positive Dimension; Negative:

Negative Dimension; Disorganized: Disorganized Dimension; Depressive: Depressive Dimension; Manic: Manic Dimension 


\section{Associations among Psychosis Dimensions: A Narrative Review}

\section{Research Question}

Although studies including the whole spectrum of functional psychotic disorders produced similar factor solutions, there is likely to be proportional variation in the dimensional profiles across different diagnostic groups, as individuals diagnosed with schizophrenia score higher in the positive, negative and disorganized factors, while patients with affective diagnoses score higher in the manic and depressive dimensions and lower in the negative and positive ones. This suggests a quantitative variation in symptom dimension scores across psychotic categories rather than qualitative differences, in which establishing differential patterns of psychosis dimension's overlap might yield further insight in the underlying mechanisms of heterogeneity in psychosis. Therefore, a narrative review of the correlations among psychopathological dimensions in subjects with a lifetime history of psychosis is presented.

\section{Searching for Factor Intercorrelation Matrixes in Clinical Psychosis}

Articles were identified through literature search based on expert advice and crossreference search. Using a start date of 1977, the following psychiatric classification systems were included: DSM-III, DSM-IIIR and DSM-IV (American Psychiatric Association, 1980, 1987, 1994), Research Diagnostic criteria (Spitzer, Endicott, \& Robins, 1978), Schedule for Affective Disorders and Schizophrenia (Endicott \& Spitzer, 1978) and ICD-9 and ICD-10 (World Health Organization, 1977, 1990b).

The following criteria guided the inclusion of studies: i) the sample consisted of patients with a lifetime history of affective and/or non-affective psychosis using a recognized criterion-based diagnostic system; ii) the study used standardized and reliable clinical scales; iii) the study conducted factor analysis with the oblique rotation method; iv) the study reported the data obtained from the factor intercorrelations matrix among psychosis dimensions; iv) the study was published as an original article in a peer-reviewed, English language journal. Given that no analytical procedures were applied, possibly overlapping samples was allowed.

The following clinical scales were used in the studies: the Brief Psychiatry Rating Scale (Overall \& Gorham, 1962), the Scale for the Assessment of Negative Symptoms (N.C. Andreasen, 1981), the Scale for the Assessment of Positive Symptoms (N.C. Andreasen, 1984), the Multidiagnostic Instrument for Psychosis (McGorry, Copolov, \& Singh, 1990; McGorry, Singh et al., 1990) and the Operational Checklist for Psychotic Disorders (McGuffin, Farmer, \& Harvey, 1991). 
The majority of factor analysis has employed the orthogonal rotation method to make the factors more interpretable. This procedure assumes that the factors are independent. However, oblique rotation methods allow the determination of inter dimension correlations, which has theoretical and empirical face validity. Furthermore, if substantial correlations are demonstrated among the factors, the intercorrelations matrix may be factor analyzed (using a varimax rotation) to obtain a second-order factor structure.

\section{Few and Inconsistent Findings}

Seven studies used rotational methods which allowed intercorrelations among pychopathological dimensions in psychosis. These studies are listed in Table 1, along with the sample sizes and the main sample characteristics, the clinical scales that were used, the statistical procedure applied, the factor solution obtained and the results of the factor intercorrelations matrixes.

\section{Disparity of Findings Leads to an Inconclusive End}

Examination of intercorrelations among psychosis dimensions in clinical samples shows inconsistent patterns of associations. Disparity in methodology was observed among the studies, which differed in their selection of measurement instruments, and inclusion of affective psychopathological indicators, which has previously been shown to influence the factor solution (Peralta \& Cuesta, 2001). Furthermore, studies tended to examine samples with different duration of disease with varying proportions of stage of disorder. Half of the studies looked exclusively at schizophrenia patients whereas the other half broadened the diagnostic concept including the whole spectrum of functional psychotic disorders. Similarly, different statistical procedures were applied. The factor solutions obtained varied from a 4-5 factor solution in most of studies to a 12 factor solution in one of them. Variability of findings in terms of factor composition may be due to the methodological disparity to the degree that an analytic synthesis of these studies could not be applied. Although psychopathological dimensions of psychosis appear to be obviously intercorrelated for both clinicians and researchers, the precise pattern of overlap can not be derived from the factor analysis literature up to date and remains uncertain. 
Table 1. Intercorrelations among Psychosis Dimensions

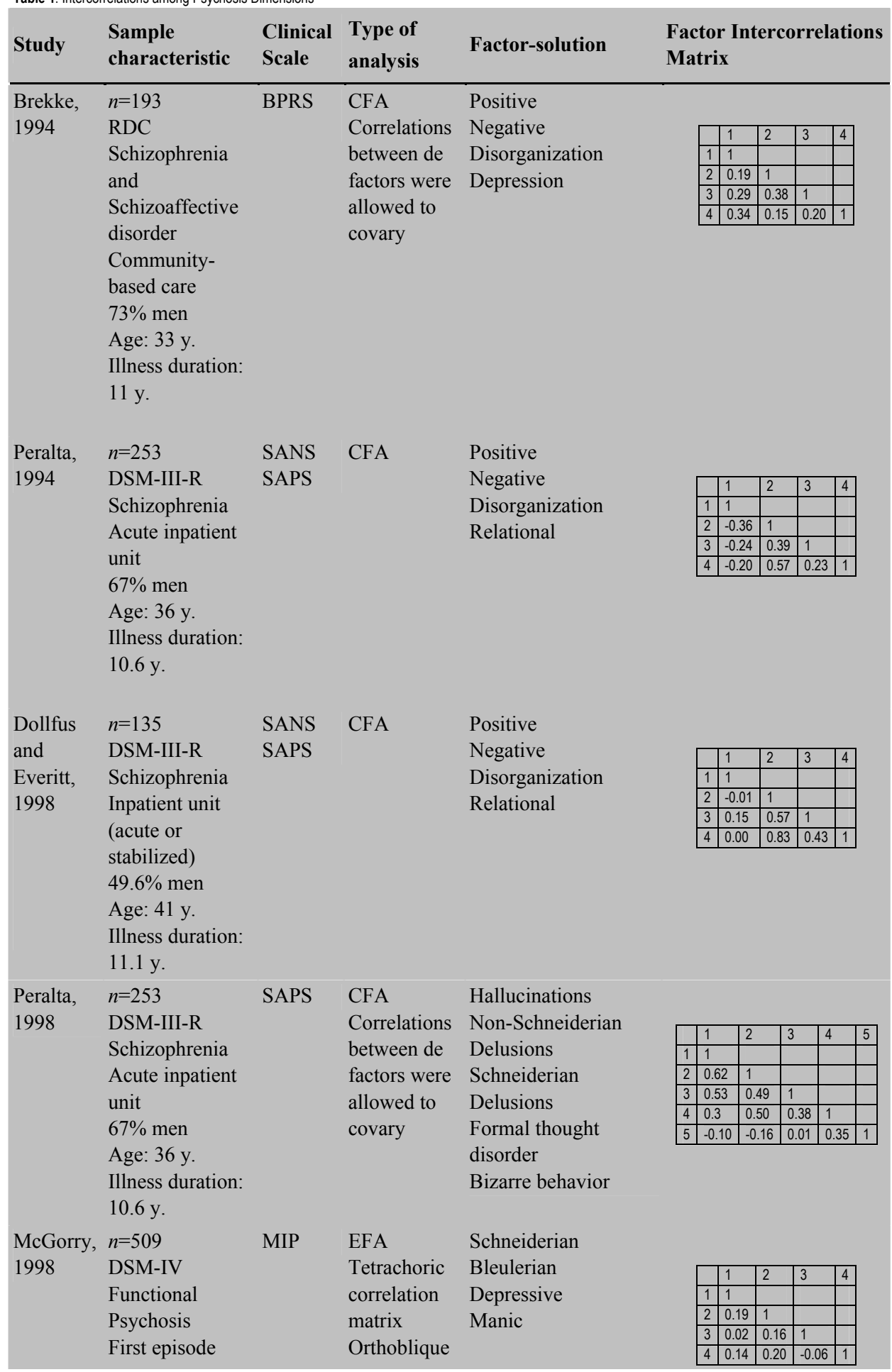




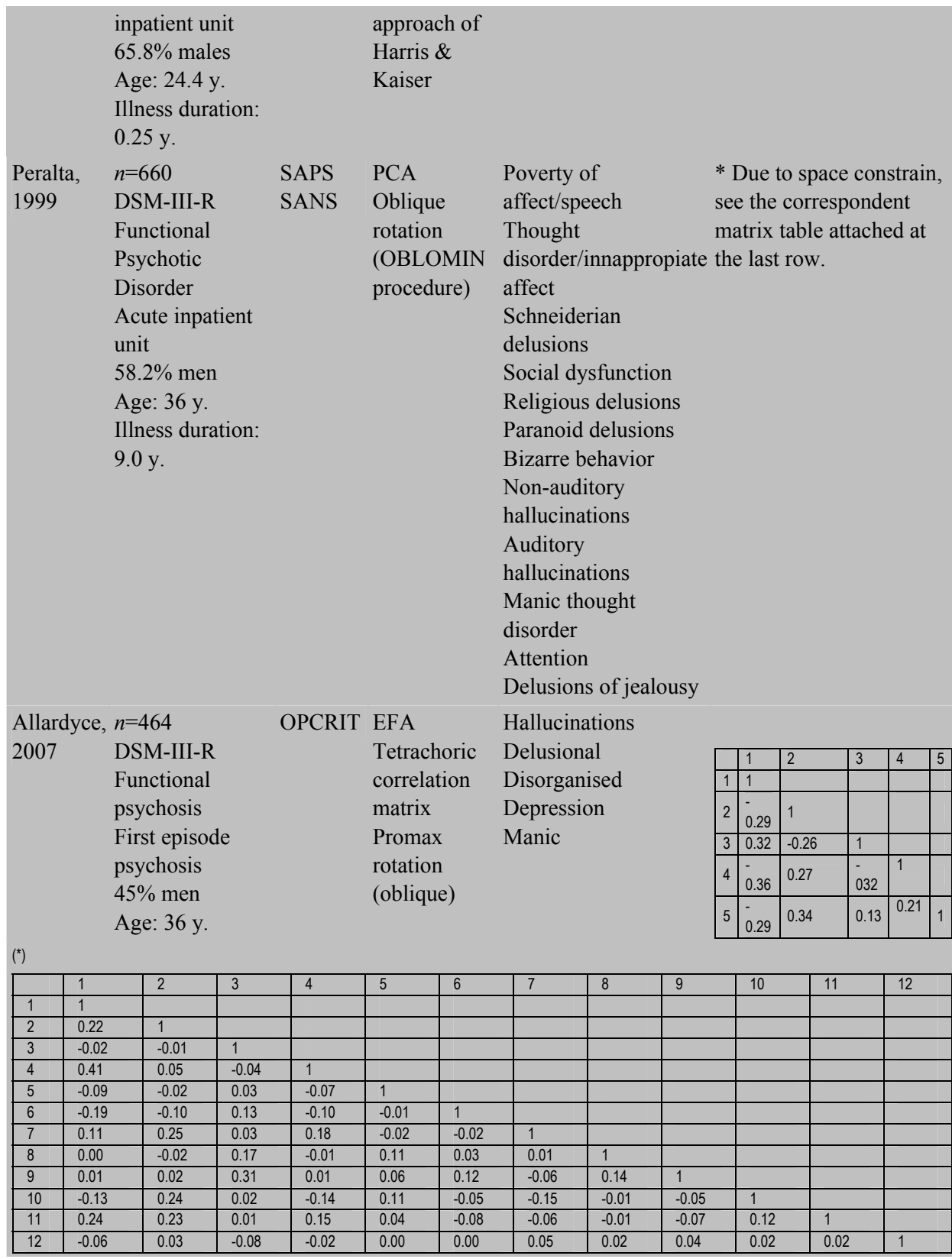

Note: n: Sample size; DSM: Diagnostic Statistical Manual; RDC: Research Diagnosis Criteria; y.: Years; BPRS: Brief Psychiatry Rating Scale; SANS: Scale for the Assessment of Negative Symptoms; SAPS: Scale for the Assessment of Positive Symptoms; MIP: Multidiagnostic Instrument for Psychosis; OPCRIT: The Operational Checklist for Psychotic Disorders; CFA: Confirmatory Factor Analysis; EFA: Exploratory Factor Analysis; PCA: Principal Component Analysis 


\section{Associations between Psychosis Dimensions and Intermediary Phenotypes: Neurocognition}

Clinical heterogeneity can be further reduced by examining intermediate phenotypes, which are considered to be more proximal to the pathophysiological processes than the symptoms and may further allow the development of hypotheses regarding the pathoplastic effects of liability to psychosis. Investigations in relatives of psychotic patients have defined intermediary phenotypes as heritable traits (Goldman et al., 2008; Greenwood et al., 2007) which underlie the illness, such as neurocognitive functioning, structural MRI brain volume measures, neurophysiological information processing traits and sensitivity to stress (Boos, Aleman, Cahn, Hulshoff Pol, \& Kahn, 2007; Bramon et al., 2005; Dickinson, Ramsey, \& Gold, 2007; Toulopoulou et al., 2007). Intermediate phenotypes are thus heritable biomarkers, lying along the causal pathway from gene to disorder, at a more proximal position to the gene than the manifest symptoms.

In particular, neurocognitive impairment is well-known as a core feature of schizophrenia, characterized by a generalized cognitive impairment with especially pronounced deficits in the domains of memory, attention, executive functioning including reasoning, concept formation, executive control and problem solving, verbal fluency, working memory, basic language processes and IQ (Aleman, Hijman, de Haan, \& Kahn, 1999; Fioravanti, Carlone, Vitale, Cinti, \& Clare, 2005; Heinrichs \& Zakzanis, 1998; Lee \& Park, 2005; Pelletier, Achim, Montoya, Lal, \& Lepage, 2005). Meta-analysis of family studies (Sitskoorn, Aleman, Ebisch, Appels, \& Kahn, 2004; Szoke et al., 2005) has demonstrated that cognitive deficiencies in relatives of schizophrenic patients parallel the deficits found in patients, and are especially pronounced in the domains of memory, executive functioning, verbal fluency, working memory and attention, in the small to moderate range. These familiar associations provide support for certain cognitive deficiencies as putative endophenotypes for schizophrenia (Lee \& Park, 2005; Sitskoorn et al., 2004).

Previously published reviews of cognitive impairment suggest that dimensions of psychopathology are not associated with neurocognitive deficits in non-affective psychotic disorder (Green, 1996), however there is some evidence suggesting subtle and contrasting associations (Kerns \& Berenbaum, 2002; Nieuwenstein, Aleman, \& de Haan, 2001) that may yield important clues to differential underlying cerebral alterations. Hundreds of studies have examined the association between psychosis psychopathology and neurocognition. Two meta-analyses (Kerns \& Berenbaum, 2002; Nieuwenstein et al., 2001) have been performed in this field. While informative, these meta-analyses were not complete, in that only non-affective dimensions and performance on few neuropsychological tests were examined. The advantage of a systematic approach including affective and non-affective measures 
of psychopathology and all measures of neurocognition in psychotic disorder would allow examining meaningful contrasts across the whole spectrum of common manifest symptoms.

\section{A Consilience Model for Clinical Psychosis}

The past decade has seen the development of inter-disciplinary working with mutual exchange and integration of ideas from the different branches of neuroscience, with the majority of psychiatric disorders now being studied from both a brain \& behavioural perspective, and models being proposed which integrate cognitive theory, phenomenology, ethiological based and pathophysiological into a single model of psychotic processes (Broome et al., 2005; Garety, Bebbington, Fowler, Freeman, \& Kuipers, 2007; van der Gaag, 2006).

In the last Lancet seminar dedicated to Schizophrenia, van Os \& Kapur (2009) formulated a consilience model for clinical psychosis incorporating the recent advances of genetics, epidemiology, imaging, cognitive sciences and psychopharmacology. This model postulates that there is a clear genetic vulnerability to schizophrenia - however, what one inherits is not the illness but altered brain development, shared in part with developmental disorders such as autism and in part with affective disorders such as bipolar disorder. In most who carry this vulnerability it only leads to subtle alterations in cognition, interpersonal style, some suspiciousness or affective dysregulation and mild functional impact. But, in some who are vulnerable - perhaps when combined with environmental insults indexed by urbanicity, cannabis and immigration - it leads to abnormal dopamine release. This abnormal dopamine release leads to an aberrant assignment of salience - thereby leading to the clinical expression of psychosis which brings these patients to attention.

In this model (Figure 2), psychotic psychopathology is differentially associated to differential distributed genetic liabilities to psychosis; (i) psychotic symptoms, shared by all diagnostic categories along the psychosis spectrum, occupy a central position in the model, as the result of gene-environment interactions integrating the neurobiological mechanism of dopamine sensitization and the cognitive process of aberrant salience attribution; (ii) developmental features such as interpersonal style and negative symptoms, associated with the intermediary phenotype of neurocognitive impairment, are the phenomenological expression of altered brain development; and (iii) affective dysregulation associated with affective genetic risk leads to the expression of reality distortion through the altered mechanism of aberrance salience attribution. To date, the epidemiological prediction of this model has not been tested. 
Figure 2. A Syndromal Consilience Model of Schizophrenia and related Psychotic Disorders; bringing together affective and non-affective dimensions of psychopathology, (GxE: gene-environment interaction). (Published in Lancet, 2009)

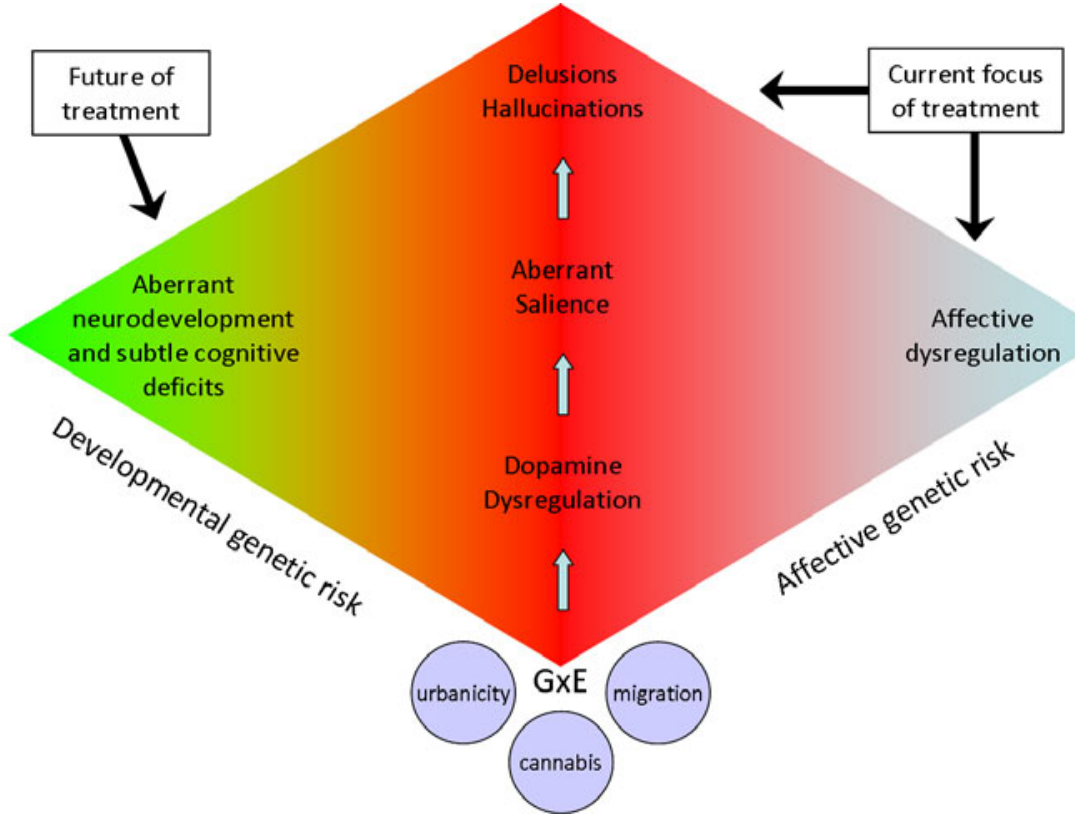

\section{Psychosis from an Epidemiological Perspective}

\section{Mapping Epidemiological Research on Psychosis in the General Population}

Psychiatric epidemiological research has developed three frameworks in terms of participants' samples: (i) clinical samples, (ii) high-risk populations, and (iii) the general population, representing alternative perspectives for the common interest of tracing the ontogenesis, development and course of psychosis. These three perspectives diverge in their target populations, the operational definition of outcomes, the variety of instruments specifically designed for each one, the different framing in terms of time and the contribution that each alternative represents.

Since the earliest psychosis research, a categorical diagnosis was developed which required -fulfilling inclusion criteria according to standardized diagnostic classifications- to be identified as a participant in a clinical sample. Once the diagnosis was established, the whole range of determinants (including symptom severity evaluated by clinical scales, i.e. the Positive and Negative Syndrome Scale (PANSS) (Kay, Fiszbein, \& Opler, 1987) and the Brief Psychiatric Rating Scale (BPRS) (Overall \& Gorham, 1962) from the natural course of the disease to the development of chronic impairment was examined. Therefore, scientific conclusions from clinical psychosis research would concern severe cases in need of 
treatment, so that one would understandably conceptualize psychosis as a discrete disease entity, as a categorical construct, distinct from normality. This, however, may not reflect the true distribution of psychosis at the population level.

High-risk populations defined groups of 'high-risk individuals' who fulfilled criteria for 'prodromal syndromes' (Hafner \& Maurer, 2006; Miller et al., 2003). Prodromal syndromes are operationally defined by characteristics which are highly correlated with the onset of the disease, including sociodemographic features, genetic risk, exposure to environmental risk factors, clusters of symptoms wellknown to precede the onset (the so-called prodromal symptoms) (Jackson, McGorry, \& Dudgeon, 1995), personality features and secondary functional impairment which will lead to clinical relevance over time. Research on High-risk populations comprises the change over time of clinically relevant characteristics assessed at frequent intervals, and resulting in high rates of transition to "psychotic disorder" over a 3- to 12- month periods (van Os \& Delespaul, 2005). Protocols for identification of high-risk populations which have demonstrated high positive predictive power (T. D. Cannon et al., 2008) can be developed yielding screening protocols, i.e. the Comprehensive Assessment of At Risk Mental State (Yung et al., 2005) and the Structure Interview for Prodromal Syndromes (Miller et al., 2003), and resulting in target groups for early intervention (Hafner et al., 2004). Given the recently reported possibility of early intervention in the course of prodromal psychotic states in ultra-high risk samples (McGorry et al., 2002), the importance of risk prediction approaches at the level of mental health services has become established (T. D. Cannon et al., 2008).

Although most research on predictors of transition to psychosis is based on clinical and high-risk samples, a third perspective faces up to investigate psychosis in large cohort studies at the level of the population. Population-based studies aim to bridge the gap between the general population and the early intervention clinic (McGorry, 2002; McGorry et al., 1995; van Os \& Delespaul, 2005). They offer the opportunity to measure psychometric risk of psychotic experiences (which can be assessed with self-reported instruments, i.e. the Self-report Symptom Checklist-90-R (L.R. Derogatis \& Cleary, 1977), the Community Assessment of Psychic Experiences (Stefanis et al., 2002) and the Composite International Diagnostic Interview (World Health Organization, 1990a)), with the potential of examining their population distribution, identifying turning points and relevant factors in the course of the very earliest expressions of psychosis and how these may develop into clinical correlates (McGorry, Yung, Bechdolf, \& Amminger, 2008). In addition, a prospective longitudinal evaluation in the general population has the capacity to examine occurrence estimates of psychometric expression and to examine their clinical correlates over time. This requires the availability of a large number of subjects to develop prediction models of a relatively low frequency outcome. 


\section{Population-based Work on Psychotic-Like Experiences}

Although psychosis has been commonly considered a rare phenomenon outside the range of normal human experience, the type of liability distribution expected for disorders with multifactorial interactive aetiologies would be continuous (Johns \& van Os, 2001; van Os, Linscott, Myin-Germeys, Delespaul, \& Krabbendam, 2009) and many mental disorders have accordingly been shown to exist along a spectrum which includes subclinical expressions, evidenced by, for example, relatively high rates of expression of bipolar spectrum symptoms, subthreshold depressive states and social restriction associated with autism (Akiskal \& Vazquez, 2006; Angst \& Cassano, 2005; Constantino \& Todd, 2003).

Similar findings have been reported for psychosis (Johns \& van Os, 2001). The clinical psychosis phenotype has not only been shown to be more prevalent than previously thought (Perala et al., 2007) but also is expressed at levels below its clinical manifestation (Chapman, Chapman, Kwapil, Eckblad, \& Zinser, 1994; Stefanis et al., 2002; van Os, Hanssen, Bijl, \& Ravelli, 2000; Yung et al., 2003). Furthermore attenuated psychopathology has been recognized and these symptoms tend to cluster in individuals and are classified as 'schizotypal' or 'schizoid' traits. A systematic review of the general population surveys suggests that the experiences associated with psychotic categories, such as paranoid delusional thinking and auditory hallucinations, are observed, in an attenuated form, in 5-8\% of respondents who have never been diagnosed with any psychotic disorder (van Os et al., 2009). Subclinical psychotic experiences are typically expressed during adolescence and in young adulthood and are usually transitory (Poulton et al., 2000). Such subclinical expressions may be the manifest expression of an underlying liability for psychotic disorders (Lataster, Myin-Germeys, Derom, Thiery, \& van Os, 2009), analogous to higher recorded blood pressure associated with a higher probability for developing future, cardiovascular disease, which shows an exposure gradient effect.

There may be continuity between the observed clinical and subclinical phenotypes since (i) similar associations with demographic factors, in particular the negative association with age and the positive association with single marital status and social disadvantage (Johns \& van Os, 2001; Peters, Joseph, \& Garety, 1999; van Os et al., 2000; Verdoux et al., 1998), (ii) sharing of environmental risk factors such as cannabis (Fergusson, Horwood, \& Swain-Campbell, 2003; Henquet et al., 2005) and strong dose-response effects of the urban environment on both phenotypes (Spauwen, Krabbendam, Lieb, Wittchen, \& van Os, 2006), (iii) sharing of cognitive and motor deficits (Krabbendam, Myin-Germeys, Hanssen, \& van Os, 2005; Voglmaier et al., 2000), (iv) sharing of risk genes (Stefanis et al., 2004) and familial co-clustering of the clinical and the subclinical expression (Kendler \& Diehl, 1993). 
Longitudinal research further shows a link between psychotic experiences in adolescence and increased probability of having a psychotic disorder later in life. Since the seminal study by Chapman and colleagues (Chapman et al., 1994), there has been replication from 2 birth cohorts (Poulton et al., 2000; Welham et al., 2009) and 2 representative general population cohorts (M. Hanssen, Bak, Bijl, Vollebergh, \& van Os, 2005; Werbeloff, Drukker, \& Dohrenwend, 2009) that low grade psychotic experiences, such as delusional thinking and mild hallucinatory experiences may precede the diagnosis of psychotic disorder, including clinical diagnosis of schizophrenia requiring hospital admission, by many years. In particular, Poulton and colleagues demonstrated that more than $25 \%$ of participants with low grade psychotic experiences at age 11 years developed a clinical psychotic disorder by age 26 years (Poulton et al., 2000). However, an equally important conclusion of that study was that the great majority of children with expression of psychotic experiences would never develop a psychotic disorder - psychosis was only a transitory developmental state for most. Similar conclusions were reached in adult population cohorts, with transition rates of around 10\% (Chapman et al., 1994; M. Hanssen et al., 2005). Cougnard and colleagues (2007) suggested that transition from transitory to persistent psychotic experiences may be due to exposure of additional environmental risk factors during significant developmental critical periods; possible environmental risk factors were cannabis use, childhood trauma and urban environment (Cougnard et al., 2007). Many questions remain, however, with regard to the hypothesized continuity over time from subclinical to clinical psychotic states.

\section{Psychometric Assessment with Self-report Instruments}

The main instruments used for the assessment of psychometric expression in the general population are self-report questionnaires, such as the Self-report Symptom Checklist-90-R (SCL-90-R) (L.R. Derogatis \& Cleary, 1977), the Community Assessment of Psychic Experiences (CAPE42) (Stefanis et al., 2002) and the Composite International Diagnostic Interview (CIDI) (World Health Organization, 1990a)).

The SCL-90-R is regarded as a comprehensive self-report symptom inventory, multidimensional in nature, and oriented toward the measurement of psychopathology in community samples of physical illnesses (Rossler et al., 2007) and also in psychiatric illness respondents (L. R. Derogatis, Rickels, \& Rock, 1976). The SCL-90-R covers a broad range of psychiatric symptoms comprising 90 items, each rated on a five-point scale of distress, which are grouped along nine primary symptom dimensions plus three global indices of pathology. Two symptom dimensions are relevant for psychosis: the six-items 'Paranoid Ideation' and the tenitems 'Psychoticism' dimensions, developed in a fashion to represent the construct 
as a continuous dimension of human experience, including the spectrum of psychotic experiences as paranoid behaviour and first-rank symptoms of schizophrenia.

The CAPE is a psychometric assessment of psychic experiences in a dimensional scale designed for use in the community. It is a 42-item self-report instrument for assessing the frequency of clinical symptoms and severity of symptom-related distress in the general population (Konings, Bak, Hanssen, van Os, \& Krabbendam, 2006; Stefanis et al., 2002; van Os et al., 2002). It evaluates three major clusters of symptoms seen in severe mental illness: positive, negative and depressive. The positive symptom dimension comprises 16 items related to delusional experiences and four items to hallucinatory experiences. Each item is rated with a 4-point Likert scale (0 to 3 ) to indicate symptom frequency ("Never", Sometimes", "Often" and "Nearly always") and a 4-point scale to indicate degree of distress experienced due to the symptom, if present ("Not distressed", "A bit distressed", "Quite distressed" and "Very distressed").

The CIDI is a comprehensive, fully standardized diagnostic interview and assesses symptoms, syndromes, and diagnoses of various mental disorders in accordance with the definitions and criteria of the ICD-10 and DSM-IV, along with information about onset, duration, severity of symptoms and psychosocial impairment. The CIDI has been primarily designed for use in epidemiological studies of mental disorders and can also be used for clinical purposes, but can be administered by trained lay people. It is divided into 16 sections: one sociodemographic section, 12 sections consisting of 288 symptom questions regarding groups of mental disorders (including as 'Somatoform and dissociative', 'Phobic and other anxiety', 'Depressive and dysthemic', 'Manic and bipolar affective', 'Schizophrenia and other psychotic', 'Eating', 'Dementia and other cognitive', 'Post-traumatic stress', as well as 'Tobacco'-, 'Alcohol'-, and 'Substance-related' disorders) and three final sections containing concluding questions, interviewer observations and interviewer ratings.

A comparison of positive psychotic experiences measured across the different instruments and emphasizing delusional and hallucinatory items is depicted in Table 2. A distinction is noted between the self-reported questionnaires SCL-90-R and CAPE 42 on the one hand, and the CIDI diagnostic interview on the other. In addition, two well-known clinical scales (the PANSS and the BPRS), specifically designed for measuring symptom severity in psychiatric patients, were included to allow comparison with a clinical, symptom-based measure. Comparing their formulation of psychosis items, self-reports on psychotic experiences can be regarded, if not as clear-cut psychotic symptoms, as a subclinical expression of psychotic experiences. 


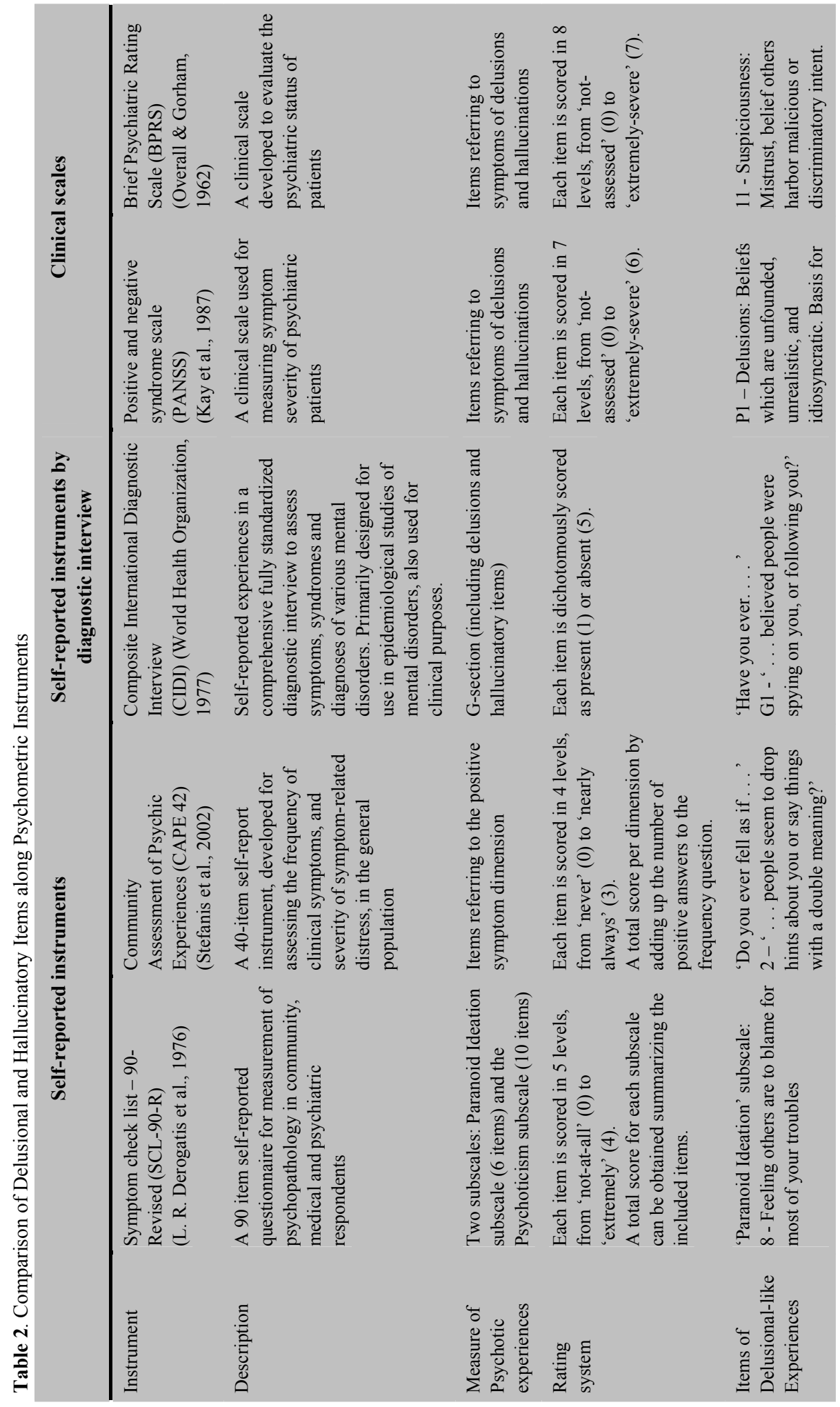




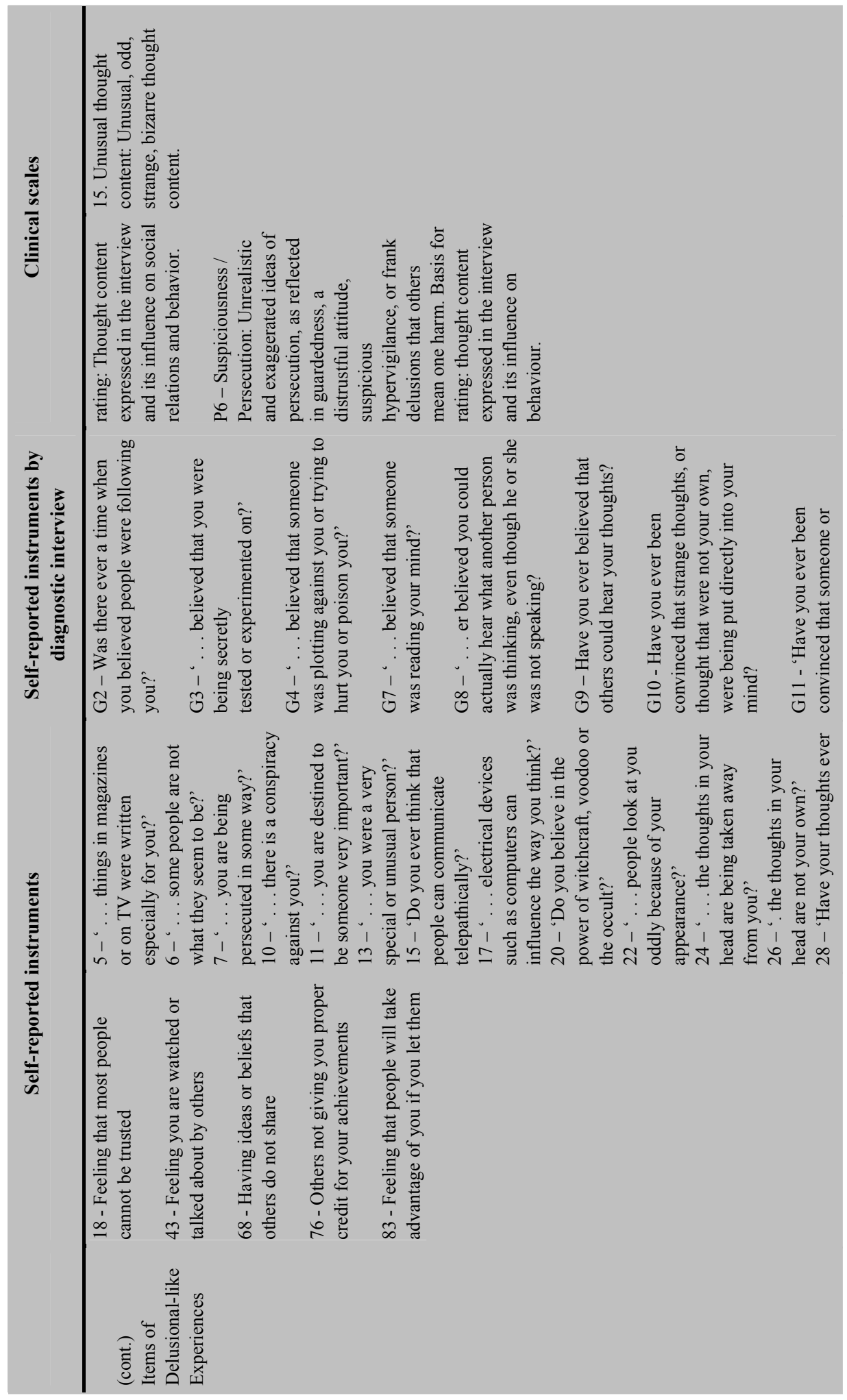







During the development of these measures of subclinical psychotic symptoms, attention has been paid to the potential measurement bias inherent in self-reported instruments. There is nevertheless a substantial literature on the (predictive or other forms of) validity of self-reported psychotic symptoms, assessed with CIDI, SCL90-R and other instruments (van Os et al., 2009). For example, one study validated self-reported psychotic symptoms against a clinical interview with the BPRS in a sample of psychotic patients, concluding that self-reported scores reflect clinical experience of psychosis (van Os, Verdoux et al., 1999). Reliability and validity of the SCL-90-R were established previously (L.R. Derogatis \& Cleary, 1977). A recent, 20-year longitudinal cohort study on psychotic symptoms also showed high predictive validity of the SCL-90-R psychosis items used in the current sample in terms of deficiencies in social achievement and functioning (Rossler et al., 2007). Validity and reliability of the CAPE 42 as a self-report instrument for the measurement of psychotic experiences in community samples has also been established (Konings et al., 2006). With regard to the CIDI, designed for use by trained interviewers who are not clinicians, it has shown high interrater (kappa $>0.70$ ) (Cottler, Helzer, Mager, Spitznagel, \& Compton, 1991; Reed, Gander, $\&$ Pfister, 1998; Wittchen, 1994; Wittchen et al., 1991). Poulton et al presented strong evidence for predictive validity of CIDI self-reported psychotic symptoms (Poulton et al., 2000). Similarly, Hanssen confirmed that the probability of having a psychotic disorder increased in a dose-response fashion with the level of selfreported psychotic experiences in the general population (M. S. Hanssen, Bijl, Vollebergh, \& van Os, 2003).

\section{Clinical Relevance versus Categorical Diagnosis}

Since the earliest clinical research on psychosis, a categorical diagnosis was considered the cut-off point to be identified as a case. While diagnosis is considered important in clinical research, it may be neither the best outcome measure nor the first step in examining the dynamic process from mental wellbeing to clinical states at a population level as it introduces a bias towards unfavourable outcomes. The diagnosis of psychotic disorders is in part understanding the onset of need for care (formal or informal) and can be assessed within the context of frequency of subclinical psychotic experiences, distress, an individual's preoccupation and how the process influences behaviour (van Os, 2009). Impairment secondary to psychotic experiences in terms of help-seeking behaviour and dysfunction makes individuals reach the status of clinical relevance. Individuals who seek professional help because of their psychotic-like experiences will invariably obtain a "formal" diagnosis, however individuals suffering from dysfunctional repercussions of psychotic experiences -i.e. dysfunction work, relationships, daily functioning and levels of distress- who do not take the initiative to seek for professional help may not reach a "formal" diagnosis. Impairment considering both, help-seeking 
behaviour and secondary dysfunction, is therefore a broader concept than categorical diagnoses of psychoses in general population samples (Poulton et al., 2000).

It is true, however, that the quality of the assessment of psychosis-related impairment in population-based studies ought to ensure (i) the quality of the assessment of psychotic experiences, (ii) the guarantee that impairment is truly secondary to those psychotic experiences, and (iii) the possibility of under/overestimating impairment. Since this is a somewhat controversial topic, it is extremely important to clarify the hazards of measuring clinical relevance of psychosis in terms of impairment at a population level. On the one hand, helpseeking behaviour may lead to an underestimation of true positives in the case of individuals who do not seek for help in spite of suffering from true clinical psychotic symptoms. On the other hand, dysfunction may lead to an overestimation of true positives in the case that individuals self-report secondary dysfunction only based on subjective level of distress. Finally, development of impairment is certainly necessary before any diagnosis of psychotic disorder can be made, and thus represents the first step that needs explaining and predicting in tracking the ontogenesis of psychotic disorder in the general population.

\section{An Epidemiological Prediction of the Clinical Psychosis Model}

The above population-based work has focused on experiences resembling positive psychotic symptoms and it is not known if and how the role of other attenuated symptom domains. Earlier general population surveys in Greece and the Netherlands have shown a high degree of overlap between psychotic experiences and affective symptoms below the threshold for clinical disorder (Krabbendam et al., 2004; Krabbendam, Myin-Germeys, Hanssen, de Graaf et al., 2005; Stefanis et al., 2002). It has also been suggested that experiences resembling negative symptoms are also expressed in attenuated form in otherwise "healthy" individuals (Maric et al., 2004).

Research in clinical samples cannot examine the degree variable clinical characteristics and other variables are due to illness or treatment effects. Similarly, clinical research cannot determine the degree to which the processes underlying symptom dimensions in psychotic patients may be universal, representing quantitative variations of normal human mentation (van Os \& Kapur, 2009) or determine if and how interacting processes underlying phenomenology, from a perspective of risk, may contribute to the onset of clinical psychosis. Therefore, epidemiological research on such psychopathological phenomena may be productive, particularly when a longitudinal perspective allows for the clarification of the direction of effects and the relationship with onset of clinical psychosis. 


\section{Aims of the Thesis}

The overall aim of this thesis was to describe the evolution of the psychotic process from mental wellness to onset of clinical psychosis within the general population.

1) To investigate the association between symptom dimensions and the intermediary phenotype of neurocognition in clinical psychosis by examining the correlations of the psychopathological dimensions and their relationship with neurocognition (an indirect measure of psychosis liability). Establishing differential patterns of cognitive performance among the different psychopathological dimensions may provide insight regarding the underlying mechanisms of heterogeneity. Since hundreds of studies had examined the association between psychosis psychopathology and neurocognition producing controversial findings, a systematic review of associations between psychopathological dimensions and neurocognition in subjects with a lifetime history of non-affective psychosis was conducted (chapter 2). The advantage of a systematic approach including affective and non-affective measures of psychopathology and all measures of neurocognition in psychotic disorder would allow examining meaningful contrasts. Previous work suggested that negative symptoms are, and positive/affective symptoms are not associated with measures of neurocognition (van Os \& Kapur, 2009), which may be compatible with two contrasting and possibly even mutually exclusive pathways to psychosis (Myin Germeys, Krabbendam, Jolles, Delespaul, \& van Os, 2002). Thus, differential patterns of neurocognition were hypothesized between the positive/affective dimensions on the one hand and the negative/disorganization dimensions on the other.

Meta-analysis is a standard method for aggregating, summarizing and drawing inferences. Associated regression methods provide the best available statistical tools for dealing with heterogeneity in the direction and strength of the association between variables. A clear set of rules is used to search for studies and to determine which studies will be included or excluded from the analysis. The results of the systematic review are based in the statistical synthesis of the data, in which the weights assigned to each study are based on mathematical criteria that are specified in advance, so that a transparent, objective and replicable framework is given for its discussion. Meta-analysis, as it is the case for the present thesis, is not only a way of synthesizing controversial information but also is part of the research process. Meta-analysis can play a role in designing new research formulating novel hypothesis based on aggregate rather than individual findings.

2) To test the dynamic process of psychosis expression through to the onset of clinical psychosis in a large representative sample of the general population followed longitudinally by examining (i) the quantitative variation of psychometric 
expressions of the psychosis phenotype (included in chapters 3, 4 and 5), (ii) the hypothesis of continuity over time from abnormal persistence of commonly transitory psychotic-like experiences to clinically relevant psychosis (chapter 3), and (iii) if and how interacting processes underlying psychopathological dimensions (the associations of which were obtained in our first aim), from a perspective of risk, may contribute to the onset of clinical psychosis (chapters 4 and 5).

To this purpose, the analyses were carried out using the prospective, observational Early Developmental Stages of Psychopathology (EDSP) study which consists on a large, general population sample of 3021 adolescents and young adults, in Munich, Germany. The EDSP study, primarily designed to examine the incidence and developmental risk factors for psychopathology, collected data on the prevalence, incidence, risk factors, comorbidity and course of mental disorders (Lieb, Isensee, von Sydow, \& Wittchen, 2000; Wittchen, Perkonigg, Lachner, \& Nelson, 1998). The overall design of this cohort study was longitudinal and prospective, consisting of a baseline and three follow-up surveys, covering a time period up to ten years.

Chapter 3 examined the degree to which positive measures of psychopathology are universal -that is, represent quantitative variations of normal human mentationand whether subclinical psychotic experiences with a greater tendency to persist over time would have a greater probability to become clinically relevant in terms of dysfunction and need for care (impairment). The hypotheses of greater persistence resulting in a greater probability of transition from subclinical to clinical psychosis characterized by impairment are tested.

Chapter 4 examined the degree to which affective measures of psychopathology are universal - that is, represent quantitative variations of normal human mentation- and if and how interacting attenuated positive and affective measures of psychopathology, from a perspective of risk, may contribute to the onset of clinical psychosis. Hypothesis were derived from evidence from clinical patient populations indicates that affective dysregulation is strongly associated with reality distortion (Myin-Germeys \& van Os, 2007; van Os \& Kapur, 2009), suggesting that a process of misassignment of emotional salience may underlie this connection. In order to test the epidemiological predictions of this model, the link between affective dysregulation, emotional salience misattribution and psychosis was examined, taking the advantage of a longitudinal perspective allowing to clarify the direction of effects and to study the relationship with onset of impairment. It was hypothesised that (i) level of affective dysregulation, in the form of depression or (hypo)mania symptoms (regardless of the presence of formal mood disorder) in the general population, would be strongly and linearly associated with experience of reality distortion, expressed as psychotic experiences, (ii) presence of 
affective dysregulation would be associated with persistence of reality distortion over time and (iii) affective dysregulation would be associated with impairment in the context of reality distortion.

In chapter 5, we examined the degree to which negative measures of psychopathology are universal - that is, represent quantitative variations of normal human mentation- and if and how interacting attenuated positive and negative measures of psychopathology, from a perspective of risk, may contribute to the onset of clinical psychosis. Hypotheses were derived from the suggestion that cognitive/negative symptoms associated with schizophrenia may represent alterations in brain development associated with distributed genetic risk, influencing a hypothesized final common pathway of neurotransmitter dysregulation resulting in the onset of positive psychotic symptoms, particularly when combined with environmental exposures, such as cannabis, developmental trauma and urbanicity (van Os \& Kapur, 2009). It was hypothesized that: (i) subclinical expressions of positive and negative symptoms are distributed in the general population and occur more often together than predicted by chance, (ii) positive symptoms are associated with environmental risks and negative features with indices of developmental impairment, (iii) negative symptoms predict psychotic experiences over time but not the other way round and, finally, (iv) "comorbid" expression of positive and negative experiences are associated with impairment and risk for patient status. 


\section{References}

Akiskal, H. S., \& Vazquez, G. H. (2006). [Widening the borders of the bipolar disorder: validation of the concept of bipolar spectrum]. Vertex, 17(69), 340-346.

Aleman, A., Hijman, R., de Haan, E. H., \& Kahn, R. S. (1999). Memory impairment in schizophrenia: A meta-analysis. American Journal of Psychiatry, 156(9), 1358-1366.

Allardyce, J., Gaebel, W., Zielasek, J., \& van Os, J. (2007). Deconstructing Psychosis conference February 2006: the validity of schizophrenia and alternative approaches to the classification of psychosis. Schizophr Bull, 33(4), 863-867.

Allardyce, J., McCreadie, R. G., Morrison, G., \& van Os, J. (2007). Do symptom dimensions or categorical diagnoses best discriminate between known risk factors for psychosis? Soc Psychiatry Psychiatr Epidemiol, 42(6), 429-437.

Allardyce, J., Suppes, T., \& van Os, J. (2007). Dimensions and the psychosis phenotype. Int J Methods Psychiatr Res, 16 Suppl 1 2007, S34-40.

American Psychiatric Association. (1980). Diagnostic and statistical manual of mental disorders (DSM-III) (3nd edn.). Washington, DC: American Psychiatric Association.

American Psychiatric Association. (1987). Diagnostic and statistical manual of mental disorders (DSM-IIIR) (3nd edn. revised). Washington, DC: American Psychiatric Association.

American Psychiatric Association. (1994). Diagnostic and statistical manual of mental disorders (DSM-IV) (4nd edn.). Washington, DC: American Psychiatric Association.

Andreasen, N. C. (1981). Scale for the assessment of negative symptoms (SANS). The University of Iowa, Iowa City. IA.

Andreasen, N. C. (1984). The scale for the assesment of positive symptoms (SAPS). The University of Iowa, Iowa City. IA.

Andreasen, N. C., \& Olsen, S. (1982). Negative v positive schizophrenia. Definition and validation. Arch Gen Psychiatry, 39(7), 789-794.

Angst, J., \& Cassano, G. (2005). The mood spectrum: improving the diagnosis of bipolar disorder. Bipolar Disord, 7 Suppl 4, 4-12.

Bleuler, E. (1950). Dementia Praecox or the Group of Schizophrenics. New York: International University Press.

Boos, H. B., Aleman, A., Cahn, W., Hulshoff Pol, H., \& Kahn, R. S. (2007). Brain volumes in relatives of patients with schizophrenia: a meta-analysis. Arch Gen Psychiatry, 64(3), 297-304.

Bramon, E., McDonald, C., Croft, R. J., Landau, S., Filbey, F., Gruzelier, J. H., et al. (2005). Is the P300 wave an endophenotype for schizophrenia? A meta-analysis and a family study. Neuroimage, 27(4), 960-968.

Brekke, J. S., DeBonis, J. A., \& Graham, J. W. (1994). A latent structure analysis of the positive and negative symptoms in schizophrenia. Compr Psychiatry, 35(4), 252-259.

Broome, M. R., Woolley, J. B., Tabraham, P., Johns, L. C., Bramon, E., Murray, G. K., et al. (2005). What causes the onset of psychosis? Schizophr Res, 79(1), 23-34.

Cannon, M., Jones, P. B., \& Murray, R. M. (2002). Obstetric complications and schizophrenia: historical and meta-analytic review. Am J Psychiatry, 159(7), 1080-1092.

Cannon, T. D., Cadenhead, K., Cornblatt, B., Woods, S. W., Addington, J., Walker, E., et al. (2008). Prediction of psychosis in youth at high clinical risk: a multisite longitudinal study in North America. Arch Gen Psychiatry, 65(1), 28-37.

Cantor-Graae, E., \& Selten, J. P. (2005). Schizophrenia and migration: a meta-analysis and review. Am J Psychiatry, 162(1), 12-24.

Carpenter, W. T., Jr., Heinrichs, D. W., \& Wagman, A. M. (1988). Deficit and nondeficit forms of schizophrenia: the concept. Am J Psychiatry, 145(5), 578-583.

Castle, D. J., Wessely, S., \& Murray, R. M. (1993). Sex and schizophrenia: effects of diagnostic stringency, and associations with and premorbid variables. Br J Psychiatry, 162, 658-664.

Chapman, L. J., Chapman, J. P., Kwapil, T. R., Eckblad, M., \& Zinser, M. C. (1994). Putatively psychosisprone subjects 10 years later. J Abnorm Psychol, 103(2), 171-183.

Constantino, J. N., \& Todd, R. D. (2003). Autistic traits in the general population: a twin study. Arch Gen Psychiatry, 60(5), 524-530.

Cottler, L. B., Helzer, J. E., Mager, D., Spitznagel, E. L., \& Compton, W. M. (1991). Agreement between DSM-III and III-R substance use disorders. Drug Alcohol Depend, 29(1), 17-25. 
Cougnard, A., Marcelis, M., Myin-Germeys, I., R, D. E. G., Vollebergh, W., Krabbendam, L., et al. (2007). Does normal developmental expression of psychosis combine with environmental risk to cause persistence of psychosis? A psychosis proneness-persistence model. Psychol Med, 37(4), 513-527.

Craddock, N., \& Owen, M. J. (2007). Rethinking psychosis: the disadvantages of a dichotomous classification now outweigh the advantages. World Psychiatry, 6(2), 84-91.

Crow, T. J. (1980). Molecular pathology of schizophrenia: more than one disease process? Br Med J, 280(6207), 66-68.

D’Souza, D. C., Perry, E., MacDougall, L., Ammerman, Y., Cooper, T., Wu, Y. T., et al. (2004). The psychotomimetic effects of intravenous delta-9-tetrahydrocannabinol in healthy individuals: implications for psychosis. Neuropsychopharmacology, 29(8), 1558-1572.

Derogatis, L. R., \& Cleary, P. A. (1977). Confirmation of the dimensional structure of the SCL-90: a study in construct validation. Journal of Clinical Psychology, 33, 981-989.

Derogatis, L. R., Rickels, K., \& Rock, A. F. (1976). The SCL-90 and the MMPI: a step in the validation of a new self-report scale. Br J Psychiatry, 128, 280-289.

Dickinson, D., Ramsey, M. E., \& Gold, J. M. (2007). Overlooking the obvious: a meta-analytic comparison of digit symbol coding tasks and other cognitive measures in schizophrenia. Arch Gen Psychiatry, 64(5), $532-542$.

Dikeos, D. G., Wickham, H., McDonald, C., Walshe, M., Sigmundsson, T., Bramon, E., et al. (2006). Distribution of symptom dimensions across Kraepelinian divisions. The British Journal of Psychiatry $189,346-353$.

Dollfus, S., \& Everitt, B. (1998). Symptom structure in schizophrenia: two-, three- or four-factor models? Psychopathology, 31(3), 120-130.

Easton, W., \& Chen, C.-Y. (2006). Epidemiology. In J. Lieberman, T. Stroup \& D. Perkins (Eds.), The American Psychiatric Publishing Textbook of Schizophrenia (pp. 17-38). Washington, DC: American Psychiatric Publishing Inc.

Endicott, J., \& Spitzer, R. L. (1978). A diagnostic interview: The schedule for affective disorders and schizophrenia. Archives of General Psychiatry, 35(7), 837-844.

Fergusson, D. M., Horwood, L. J., \& Swain-Campbell, N. R. (2003). Cannabis dependence and psychotic symptoms in young people. Psychol Med, 33(1), 15-21.

Fioravanti, M., Carlone, O., Vitale, B., Cinti, M. E., \& Clare, L. (2005). A meta-analysis of cognitive deficits in adults with a diagnosis of schizophrenia. Neuropsychology Review, 15(2), 73-95.

Garety, P. A., Bebbington, P., Fowler, D., Freeman, D., \& Kuipers, E. (2007). Implications for neurobiological research of cognitive models of psychosis: a theoretical paper. Psychol Med, 37(10), 1377-1391.

Goldman, A. L., Pezawas, L., Mattay, V. S., Fischl, B., Verchinski, B. A., Zoltick, B., et al. (2008). Heritability of brain morphology related to schizophrenia: a large-scale automated magnetic resonance imaging segmentation study. Biol Psychiatry, 63(5), 475-483.

Good, K. P., Rabinowitz, J., Whitehorn, D., Harvey, P. D., DeSmedt, G., \& Kopala, L. C. (2004). The relationship of neuropsychological test performance with the PANSS in antipsychotic naive, firstepisode psychosis patients. Schizophrenia Research, 68(1), 11-19.

Green, M. F. (1996). What are the functional consequences of neurocognitive deficits in schizophrenia? American Journal of Psychiatry, 153(3), 321-330.

Greenwood, T. A., Braff, D. L., Light, G. A., Cadenhead, K. S., Calkins, M. E., Dobie, D. J., et al. (2007). Initial heritability analyses of endophenotypic measures for schizophrenia: the consortium on the genetics of schizophrenia. Arch Gen Psychiatry, 64(11), 1242-1250.

Grube, B. S., Bilder, R. M., \& Goldman, R. S. (1998). Meta-analysis of symptom factors in schizophrenia. Schizophrenia Research, 31(2-3), 113-120.

Hafner, H., \& Maurer, K. (2006). Early detection of schizophrenia: current evidence and future perspectives. World Psychiatry, 5(3), 130-138.

Hafner, H., Maurer, K., Ruhrmann, S., Bechdolf, A., Klosterkotter, J., Wagner, M., et al. (2004). Early detection and secondary prevention of psychosis: facts and visions. Eur Arch Psychiatry Clin Neurosci, 254(2), 117-128.

Hanssen, M., Bak, M., Bijl, R., Vollebergh, W., \& van Os, J. (2005). The incidence and outcome of subclinical psychotic experiences in the general population. Br J Clin Psychol, 44(Pt 2), 181-191. 
Hanssen, M. S., Bijl, R. V., Vollebergh, W., \& van Os, J. (2003). Self-reported psychotic experiences in the general population: a valid screening tool for DSM-III-R psychotic disorders? Acta Psychiatr Scand, 107(5), 369-377.

Harrison, P. J., \& Owen, M. J. (2003). Genes for schizophrenia? Recent findings and their pathophysiological implications. Lancet, 361(9355), 417-419.

Heinrichs, R. W., \& Zakzanis, K. K. (1998). Neurocognitive deficit in schizophrenia: A quantitative review of the evidence. Neuropsychology, 12(3), 426-445.

Henquet, C., Krabbendam, L., Spauwen, J., Kaplan, C., Lieb, R., Wittchen, H. U., et al. (2005). Prospective cohort study of cannabis use, predisposition for psychosis, and psychotic symptoms in young people. Bmj, 330(7481), 11.

Jackson, H. J., McGorry, P. D., \& Dudgeon, P. (1995). Prodromal symptoms of schizophrenia in first-episode psychosis: prevalence and specificity. Compr Psychiatry, 36(4), 241-250.

Johns, L. C., \& van Os, J. (2001). The continuity of psychotic experiences in the general population. Clin Psychol Rev, 21(8), 1125-1141.

Kay, S. R. (1990). Significance of the positive-negative distinction in schizophrenia. Schizophr Bull, 16(4), 635-652.

Kay, S. R., Fiszbein, A., \& Opler, L. A. (1987). The positive and negative syndrome scale (PANSS) for schizophrenia. Schizophrenia Bulletin, 13(2), 261-276.

Kendler, K. S., \& Diehl, S. R. (1993). The genetics of schizophrenia: a current, genetic-epidemiologic perspective. Schizophr Bull, 19(2), 261-285.

Kerns, J. G., \& Berenbaum, H. (2002). Cognitive impairments associated with formal thought disorder in people with schizophrenia. Journal of Abnormal Psychology, 111(2), 211-224.

Konings, M., Bak, M., Hanssen, M., van Os, J., \& Krabbendam, L. (2006). Validity and reliability of the CAPE: a self-report instrument for the measurement of psychotic experiences in the general population. Acta Psychiatr Scand, 114(1), 55-61.

Krabbendam, L., Myin-Germeys, I., De Graaf, R., Vollebergh, W., Nolen, W. A., Iedema, J., et al. (2004). Dimensions of depression, mania and psychosis in the general population. Psychol Med, 34(7), 11771186.

Krabbendam, L., Myin-Germeys, I., Hanssen, M., de Graaf, R., Vollebergh, W., Bak, M., et al. (2005). Development of depressed mood predicts onset of psychotic disorder in individuals who report hallucinatory experiences. Br J Clin Psychol, 44(Pt 1), 113-125.

Krabbendam, L., Myin-Germeys, I., Hanssen, M., \& van Os, J. (2005). Familial covariation of the subclinical psychosis phenotype and verbal fluency in the general population. Schizophr Res, 74(1), 37-41.

Krabbendam, L., \& van Os, J. (2005). Schizophrenia and urbanicity: a major environmental influence-conditional on genetic risk. Schizophr Bull, 31(4), 795-799.

Kraepelin. (1919). Dementia Praecox and Paraphrenia (translated by M. Barclay). Churchill Livingstone, Edinburgh.

Lataster, T., Myin-Germeys, I., Derom, C., Thiery, E., \& van Os, J. (2009). Evidence that self-reported psychotic experiences represent the transitory developmental expression of genetic liability to psychosis in the general population. Am J Med Genet B Neuropsychiatr Genet.

Lee, J., \& Park, S. (2005). Working memory impairments in schizophrenia: A meta-analysis. Journal of Abnormal Psychology, 114(4), 599-611.

Liddle, P. F. (1992). Signs and symptoms of psychotic illness. Hammersmith Hospital, London. (Questionnaire available from the author on request.).

Lieb, R., Isensee, B., von Sydow, K., \& Wittchen, H. U. (2000). The Early Developmental Stages of Psychopathology Study (EDSP): a methodological update. Eur Addict Res, 6(4), 170-182.

Lindenmayer, J. P., Brown, E., Baker, R. W., Schuh, L. M., Shao, L., Tohen, M., et al. (2004). An excitement subscale of the Positive and Negative Syndrome Scale. Schizophr Res, 68(2-3), 331-337.

Lindenmayer, J. P., Grochowski, S., \& Hyman, R. B. (1995). Five factor model of schizophrenia: replication across samples. Schizophr Res, 14(3), 229-234.

Maric, N., Myin-Germeys, I., Delespaul, P., de Graaf, R., Vollebergh, W., \& van Os, J. (2004). Is our concept of schizophrenia influenced by Berkson's bias? Soc Psychiatry Psychiatr Epidemiol, 39(8), 600-605.

McGorry, P. D. (2002). The recognition and optimal management of early psychosis: an evidence-based reform. World Psychiatry, 1(2), 76-83. 
McGorry, P. D., Bell, R. C., Dudgeon, P. L., \& Jackson, H. J. (1998). The dimensional structure of first episode psychosis: an exploratory factor analysis. Psychological Medicine, 28(4), 935-947.

McGorry, P. D., Copolov, D. L., \& Singh, B. S. (1990). Royal Park Multidiagnostic Instrument for Psychosis: Part I. Rationale and review. Schizophr Bull, 16(3), 501-515.

McGorry, P. D., McFarlane, C., Patton, G. C., Bell, R., Hibbert, M. E., Jackson, H. J., et al. (1995). The prevalence of prodromal features of schizophrenia in adolescence: a preliminary survey. Acta Psychiatr Scand, 92(4), 241-249.

McGorry, P. D., Singh, B. S., Copolov, D. L., Kaplan, I., Dossetor, C. R., \& van Riel, R. J. (1990). Royal Park Multidiagnostic Instrument for Psychosis: Part II. Development, reliability, and validity. Schizophr Bull, 16(3), 517-536.

McGorry, P. D., Yung, A. R., Bechdolf, A., \& Amminger, P. (2008). Back to the future: predicting and reshaping the course of psychotic disorder. Arch Gen Psychiatry, 65(1), 25-27.

McGorry, P. D., Yung, A. R., Phillips, L. J., Yuen, H. P., Francey, S., Cosgrave, E. M., et al. (2002). Randomized controlled trial of interventions designed to reduce the risk of progression to first-episode psychosis in a clinical sample with subthreshold symptoms. Arch Gen Psychiatry, 59(10), 921-928.

McGrath, J., Saha, S., Chant, D., \& Welham, J. (2008). Schizophrenia: a concise overview of incidence, prevalence, and mortality. Epidemiol Rev, 30, 67-76.

McGuffin, P., Farmer, A., \& Harvey, I. (1991). A polydiagnostic application of operational criteria in studies of psychotic illness. Development and reliability of the OPCRIT system. Arch Gen Psychiatry, 48(8), 764-770.

Menezes, N. M., Arenovich, T., \& Zipursky, R. B. (2006). A systematic review of longitudinal outcome studies of first-episode psychosis. Psychol Med, 36(10), 1349-1362.

Miller, T. J., McGlashan, T. H., Rosen, J. L., Cadenhead, K., Cannon, T., Ventura, J., et al. (2003). Prodromal assessment with the structured interview for prodromal syndromes and the scale of prodromal symptoms: predictive validity, interrater reliability, and training to reliability. Schizophr Bull, 29(4), 703-715.

Morgan, C., Kirkbride, J., Hutchinson, G., Craig, T., Morgan, K., Dazzan, P., et al. (2008). Cumulative social disadvantage, ethnicity and first-episode psychosis: a case-control study. Psychol Med, 38(12), 17011715 .

Morrison, A. P., Frame, L., \& Larkin, W. (2003). Relationships between trauma and psychosis: a review and integration. Br J Clin Psychol, 42(Pt 4), 331-353.

Murray, C., \& Lopez, A. (1996). The global burden of disease and injury series, vol 1: a comprehensive assessment of mortality and disability from diseases, injuries, and risk factors in 1990 and projected to 2020. Cambridge, MA: Harvard University Press.

Myin-Germeys, I., \& van Os, J. (2007). Stress-reactivity in psychosis: evidence for an affective pathway to psychosis. Clin Psychol Rev, 27(4), 409-424.

Myin Germeys, I., Krabbendam, L., Jolles, J., Delespaul, P. A., \& van Os, J. (2002). Are cognitive impairments associated with sensitivity to stress in schizophrenia? An experience sampling study. American Journal of Psychiatry, 159(3), 443-449.

Nieuwenstein, M. R., Aleman, A., \& de Haan, E. H. (2001). Relationship between symptom dimensions and neurocognitive functioning in schizophrenia: A meta-analysis of WCST and CPT studies. Wisconsin Card Sorting Test. Continuous Performance Test. Journal of Psychiatric Research, 35(2), 119-125.

Overall, J. E., \& Gorham, D. R. (1962). The brief psychiatric rating scale. Psychological Reports, 10, 799812.

Pelletier, M., Achim, A. M., Montoya, A., Lal, S., \& Lepage, M. (2005). Cognitive and clinical moderators of recognition memory in schizophrenia: A meta-analysis. Schizophrenia Research, 74(2-3), 233-252.

Perala, J., Suvisaari, J., Saarni, S. I., Kuoppasalmi, K., Isometsa, E., Pirkola, S., et al. (2007). Lifetime prevalence of psychotic and bipolar I disorders in a general population. Arch Gen Psychiatry, 64(1), 1928.

Peralta, V., \& Cuesta, M. J. (1998). Factor structure and clinical validity of competing models of positive symptoms in schizophrenia. Biol Psychiatry, 44(2), 107-114.

Peralta, V., \& Cuesta, M. J. (1999). Dimensional structure of psychotic symptoms: an item-level analysis of SAPS and SANS symptoms in psychotic disorders. Schizophr Res, 38(1), 13-26.

Peralta, V., \& Cuesta, M. J. (2001). How many and which are the psychopathological dimensions in schizophrenia? Issues influencing their ascertainment. Schizophr Res, 49(3), 269-285. 
Peralta, V., Cuesta, M. J., \& de Leon, J. (1994). An empirical analysis of latent structures underlying schizophrenic symptoms: A four-syndrome model. Biological Psychiatry, 36(11), 726-736.

Peters, E. R., Joseph, S. A., \& Garety, P. A. (1999). Measurement of delusional ideation in the normal population: introducing the PDI (Peters et al. Delusions Inventory). Schizophr Bull, 25(3), 553-576.

Poulton, R., Caspi, A., Moffitt, T. E., Cannon, M., Murray, R., \& Harrington, H. (2000). Children's selfreported psychotic symptoms and adult schizophreniform disorder: a 15 -year longitudinal study. Arch Gen Psychiatry, 57(11), 1053-1058.

Reed, V., Gander, F., \& Pfister, H. (1998). To what degree does the Composite International Diagnostic Interview (CID) correctly identify DSM-IV disorders? Testing validity issues in a clinical sample. . International Journal of Methods in Psychiatric Research, 7, 142-155.

Rossler, W., Riecher-Rossler, A., Angst, J., Murray, R., Gamma, A., Eich, D., et al. (2007). Psychotic experiences in the general population: a twenty-year prospective community study. Schizophr Res, 92(13), 1-14.

Roy, M. A., Maziade, M., Labbe, A., \& Merette, C. (2001). Male gender is associated with deficit schizophrenia: a meta-analysis. Schizophr Res, 47(2-3), 141-147.

Schneider, K. (1959). Clinical Psychopathology (translated M.W. Hamilton) (5th edn). New York: Grune \& Stratton.

Serretti, A., \& Olgiati, P. (2004). Dimensions of major psychoses: a confirmatory factor analysis of six competing models. Psychiatry Res, 127(1-2), 101-109.

Sitskoorn, M. M., Aleman, A., Ebisch, S. J., Appels, M. C., \& Kahn, R. S. (2004). Cognitive deficits in relatives of patients with schizophrenia: A meta-analysis. Schizophrenia Research, 71(2-3), 285-295.

Spauwen, J., Krabbendam, L., Lieb, R., Wittchen, H. U., \& van Os, J. (2006). Evidence that the outcome of developmental expression of psychosis is worse for adolescents growing up in an urban environment. Psychol Med, 36(3), 407-415.

Spitzer, R. L., Endicott, J., \& Robins, E. (1978). Research diagnostic criteria: Rationale and reliability. Archives of General Psychiatry, 35(6), 773-782.

Stefanis, N. C., Hanssen, M., Smirnis, N. K., Avramopoulos, D. A., Evdokimidis, I. K., Stefanis, C. N., et al. (2002). Evidence that three dimensions of psychosis have a distribution in the general population. Psychol Med, 32(2), 347-358.

Stefanis, N. C., van Os, J., Avramopoulos, D., Smyrnis, N., Evdokimidis, I., Hantoumi, I., et al. (2004). Variation in catechol-o-methyltransferase val158 met genotype associated with schizotypy but not cognition: a population study in 543 young men. Biol Psychiatry, 56(7), 510-515.

Szoke, A., Schurhoff, F., Mathieu, F., Meary, A., Ionescu, S., \& Leboyer, M. (2005). Tests of executive functions in first-degree relatives of schizophrenic patients: A meta-analysis. Psychological Medicine, 35(6), 771-782.

Toulopoulou, T., Picchioni, M., Rijsdijk, F., Hua-Hall, M., Ettinger, U., Sham, P., et al. (2007). Substantial genetic overlap between neurocognition and schizophrenia: genetic modeling in twin samples. Arch Gen Psychiatry, 64(12), 1348-1355.

van der Gaag, M. (2006). A neuropsychiatric model of biological and psychological processes in the remission of delusions and auditory hallucinations. Schizophr Bull, 32 Suppl 1, S113-122.

van Os, J. (2009). A salience dysregulation syndrome. Br J Psychiatry, 194(2), 101-103.

van Os, J., \& Allardyce, J. (2009). The Clinical Epidemiology of Schizophrenia. In B. Kaplan, J. Saddock, V. Sadock \& P. Ruiz (Eds.), Kaplan \& Sadock’s Comprehensive Textbook of Psychiatry, Ninth Edition (pp. in press). London: Lippincott Williams \& Wilkins

van Os, J., Bak, M., Hanssen, M., Bijl, R. V., de Graaf, R., \& Verdoux, H. (2002). Cannabis use and psychosis: a longitudinal population-based study. Am J Epidemiol, 156(4), 319-327.

van Os, J., \& Delespaul, P. (2005). Toward a world consensus on prevention of schizophrenia. Dialogues Clin Neurosci, 7(1), 53-67.

van Os, J., Gilvarry, C., Bale, R., Van Horn, E., Tattan, T., White, I., et al. (1999). A comparison of the utility of dimensional and categorical representations of psychosis. UK700 Group. Psychol Med, 29(3), 595606.

van Os, J., Hanssen, M., Bijl, R. V., \& Ravelli, A. (2000). Strauss (1969) revisited: a psychosis continuum in the general population? Schizophr Res, 45(1-2), 11-20.

van Os, J., \& Kapur, S. (2009). Schizophrenia. Lancet, 374(9690), 635-645. 
van Os, J., Linscott, R. J., Myin-Germeys, I., Delespaul, P., \& Krabbendam, L. (2009). A systematic review and meta-analysis of the psychosis continuum: evidence for a psychosis proneness-persistenceimpairment model of psychotic disorder. Psychol Med, 39(2), 179-195.

van Os, J., \& Marcelis, M. (1998). The ecogenetics of schizophrenia: a review. Schizophr Res, 32(2), 127135.

van Os, J., Verdoux, H., Maurice-Tison, S., Gay, B., Liraud, F., Salamon, R., et al. (1999). Self-reported psychosis-like symptoms and the continuum of psychosis. Soc Psychiatry Psychiatr Epidemiol, 34(9), 459-463.

Verdoux, H., van Os, J., Maurice-Tison, S., Gay, B., Salamon, R., \& Bourgeois, M. (1998). Is early adulthood a critical developmental stage for psychosis proneness? A survey of delusional ideation in normal subjects. Schizophr Res, 29(3), 247-254.

Voglmaier, M. M., Seidman, L. J., Niznikiewicz, M. A., Dickey, C. C., Shenton, M. E., \& McCarley, R. W. (2000). Verbal and nonverbal neuropsychological test performance in subjects with schizotypal personality disorder. Am J Psychiatry, 157(5), 787-793.

Welham, J., Scott, J., Williams, G., Najman, J., Bor, W., O’Callaghan, M., et al. (2009). Emotional and behavioural antecedents of young adults who screen positive for non-affective psychosis: a 21-year birth cohort study. Psychol Med, 39(4), 625-634.

Werbeloff, N., Drukker, M., \& Dohrenwend, B. (2009). Self-Reported Psychotic Symptoms in the Community are Associated with Increased Risk of Later Hospitalization for Non-Affective Psychotic Disorders. In C. Abstract (Ed.) (Vol. 35, pp. 74): Schizophrenia Bulletin.

Wittchen, H. U. (1994). Reliability and validity studies of the WHO--Composite International Diagnostic Interview (CIDI): a critical review. J Psychiatr Res, 28(1), 57-84.

Wittchen, H. U., Perkonigg, A., Lachner, G., \& Nelson, C. B. (1998). Early developmental stages of psychopathology study (EDSP): objectives and design. Eur Addict Res, 4(1-2), 18-27.

Wittchen, H. U., Robins, L. N., Cottler, L. B., Sartorius, N., Burke, J. D., \& Regier, D. (1991). Cross-cultural feasibility, reliability and sources of variance of the Composite International Diagnostic Interview (CIDI). The Multicentre WHO/ADAMHA Field Trials. Br J Psychiatry, 159, 645-653, 658.

World Health Organization. (1977). International classification of diseases (9th revision). Ginebra: World Health Organization.

World Health Organization. (1990a). Composite International Diagnostic Interview (CIDI), Version 1.0. Geneva: WHO.

World Health Organization. (1990b). International classification of diseases (10th revision). Ginebra: World Health Organization.

Yung, A. R., Phillips, L. J., Yuen, H. P., Francey, S. M., McFarlane, C. A., Hallgren, M., et al. (2003). Psychosis prediction: 12-month follow up of a high-risk ("prodromal") group. Schizophr Res, 60(1), 2132.

Yung, A. R., Yuen, H. P., McGorry, P. D., Phillips, L. J., Kelly, D., Dell’Olio, M., et al. (2005). Mapping the onset of psychosis: the Comprehensive Assessment of At-Risk Mental States. Aust N Z J Psychiatry, 39(11-12), 964-971. 



\section{CHAPTER 2}

\section{ARE PSYCHOTIC PSYCHOPATHOLOGY AND NEUROCOGNITION ORTHOGONAL? A SYSTEMATIC REVIEW OF THEIR ASSOCIATIONS}

Maria de Gracia Dominguez ${ }^{1}$, Wolfgang Viechtbauer ${ }^{2}$, Claudia J.P. Simons ${ }^{1}$, Jim van Os ${ }^{1,3}$, Lydia Krabbendam $^{1}$

${ }^{1}$ Department of Psychiatry and Neuropsychology, South Limburg Mental Health Research and Teaching Network, EURON, Maastricht University, MD Maastricht, The Netherlands.

${ }^{2}$ Department of Methodology and Statistics, Maastricht University, Maastricht, The Netherlands.

${ }^{3}$ Division of Psychological Medicine, Institute of Psychiatry, London, UK.

Psychological Bulletin, 2009.Vol.135, Iss.1. pages 157-171 


\section{Abstract \\ Objective and Method}

A systematic review (58 studies, 5009 individuals) is presented of associations between psychopathological dimensions of psychosis and measures of neurocognitive impairment in subjects with a lifetime history of nonaffective psychosis.

\section{Results}

Results showed that negative and disorganized dimensions were significantly but modestly associated with cognitive deficits (correlations ranging from -.29 to -.12). In contrast, positive and depressive dimensions of psychopathology were not associated with neurocognitive measures. The patterns of association for the 4 psychosis dimensions were stable across neurocognitive domains and independent of age, gender and chronicity of illness. In addition, significantly higher correlations were found for the negative dimension in relation to verbal fluency (p: .005) and for the disorganized dimension in relation to reasoning and problem solving (p: .004) and attention/vigilance ( $\mathrm{p}$-value: .03).

\section{Conclusions}

Psychotic psychopathology and neurocognition are not entirely orthogonal, as heterogeneity in nonaffective psychosis is weakly but meaningfully associated with measures of neurocognition. This association suggests that differential latent cerebral mechanisms underlie the cluster of disorganized and negative symptoms versus that of positive and affective symptoms.

\section{Key words}

Meta-analysis, Systematic Review, Psychosis Dimensions, Cognitive Disorders, Neurocognition 


\section{Introduction}

Measures of psychopathology define the diagnostic phenotype in nonaffective psychotic disorder (Dikeos et al., 2006; Grube, Bilder, \& Goldman, 1998; Lindenmayer, Grochowski, \& Hyman, 1995; McGorry, Bell, Dudgeon, \& Jackson, 1998). Cognitive impairment is an important feature of psychotic disorder (Fioravanti, Carlone, Vitale, Cinti, \& Clare, 2005; Heinrichs \& Zakzanis, 1998) that reflects developmental impairment and expression of genetic risk (Nuechterlein \& Dawson, 1984; Sitskoorn, Aleman, Ebisch, Appels, \& Kahn, 2004; Strauss, Buchanan, \& Hale, 1993; Szoke et al., 2005). Although it has been suggested that cognitive impairment is not associated with dimensions of psychopathology in nonaffective psychotic disorder (Green, 1996), there is evidence suggesting subtle and contrasting associations (Kerns \& Berenbaum, 2002; Nieuwenstein, Aleman, \& de Haan, 2001) that may yield important clues to differential underlying cerebral alterations.

Two meta-analyses have been performed in this field. The meta-analysis by Nieuwenstein et al (2001) provided a quantitative review of the relations between three dimensions of schizophrenia symptoms (negative and positive dimensions, the latter subdivided into disorganization and reality distortion) and performance on 2 neuropsychological tests related to executive function and vigilance: the Wisconsin Card Sorting Test (WCST; 16 studies) and the Continuous Performance Test (CPT; 6 studies), respectively. Negative symptoms were significantly associated with impairments in both domains of cognitive functioning. Disorganization showed a significant association with worse WCST performance but not with CPT performance. Although significant, the effect sizes were small to modest in magnitude. General scores for all positive symptoms and separate scores for reality distortion symptoms were not associated with either WCST or CPT performance. Moderator analyses indicated that neither age, nor gender, nor duration of illness affected the overall effect sizes. Another meta-analysis, reported by Kerns and Berenbaum (2002), was focused exclusively on formal thought disorder and examined which of four specific cognitive domains would be associated with this psychopathological measure. Kerns and Berenbaum concluded that impaired executive functioning (26 studies) and impaired semantic memory (8 studies) were consistently associated but that increased spreading activation (5 studies) and impaired language production ( 5 studies) were not. Although informative, these meta-analyses were not complete, in that many other studies in nonaffective psychosis have assessed associations between domains of neurocognition and psychopathology (in particular, depression) that they did not cover.

The advantage of a systematic approach that includes affective measures of psychopathology and all measures of neurocognition is that such an approach 
allows examination of meaningful contrasts. Previous work (Myin Germeys, Krabbendam, Jolles, Delespaul, \& van Os, 2002) has suggested that negative symptoms are and positive/affective symptoms are not associated with measures of neurocognition, and this finding may be compatible with two contrasting and possibly even mutually exclusive pathways to psychosis. Therefore, establishing differential patterns of cognitive performance among the different psychopathological dimensions may yield further insight into the underlying mechanisms of heterogeneity of psychosis. Thus, differential patterns of neurocognition were hypothesized between the positive/affective dimensions on the one hand and the negative/disorganization dimensions on the other.

\section{Method}

\section{Data Sources and Literature Search}

Articles were identified through a literature search in MEDLINE and PsychINFO, the latter of which also references unpublished works such as those found in Dissertations Abstracts. The search covered the period from January 1977 to April 2008. We used a start date of 1977 and included the following psychiatric classification systems: Diagnostic and Statistical Manual of Mental Disorders (American Psychiatric Association, 1980, 1987, 1994); Research Diagnostic Criteria (Spitzer, Endicott, \& Robins, 1978), Schedule for Affective Disorders and Schizophrenia (Endicott \& Spitzer, 1978) and International Classification of Diseases (World Health Organization, 1977, 1990). The keywords were psychosis or schizophrenia, combined with neuropsychol* or neurocogn*, combined with positive or negative or disorg* or depressive. The search produced 3,098 articles. References provided by relevant meta-analyses (Kerns \& Berenbaum, 2002; Nieuwenstein et al., 2001) and included in the retrieved articles were examined and yielded an additional 31 articles. We contacted the authors of the 15 unpublished dissertation abstracts that were potentially eligible for inclusion in order to retrieve the necessary data. One author responded and referred to a later publication in which all relevant data were reported. Therefore, the abstracts of 3,129 articles were examined for possible inclusion in the analysis.

\section{Criteria for Inclusion and Exclusion}

The following criteria guided the inclusion of studies in the meta-analysis: (a) the sample consisted of patients with a lifetime history of nonaffective psychosis according a recognized criterion-based diagnostic system; (b) the study used standardized and reliable clinical scales and neuropsychological tasks; (c) the study reported all the correlations between symptom dimensions and neuropsychological performance; (d) the study was published as an original article in a peer-reviewed, 
English-language journal. Studies that included groups of patients with special characteristics that possibly affected neuropsychological performance (e.g., geriatric patients or patients with childhood psychosis) were excluded.

\section{Overlapping of Samples}

The studies that fulfilled the inclusion criteria were examined for possibly overlapping samples. Authors whose studies were performed in the same departments or areas were contacted and were asked to provide the relevant information. Any overlap was dealt with in one of three different ways: (a) Of the studies with complete overlap in samples and clinical/cognitive measures, the study with the smaller sample size was excluded. This was the case for Good et al. (2004) versus Heydebrand et al. (2004); Docherty and Gordinier (1999) versus A. S. Cohen and Docherty (2004), and J. Addington and Addington (1998) versus J. Addington \& Addington (1997). (b) Of the pairs of studies with overlap in samples but without overlap in the examined associations between cognitive domains and psychopathological dimensions, both studies were included, but the size of the smallest sample from each pair was subtracted from the total number of individuals who contributed to the meta-analysis. This was the case for Woodward, Ruff, Thornton, Moritz, \& Liddle (2003) versus Woodward, Thornton, Ruff, Moritz, and Liddle (2004) and O'Leary et al. (2000) versus Torres, O'Leary, \& Andreasen (2004). (c) Of the pairs of studies in which the degree of overlap in the samples was uncertain, all the studies were initially included in the analysis, and sensitivity analysis were conducted in which the studies with the smallest samples of each pair were excluded. This was the case for Braff et al. (1991) versus Perry and Braff (1998) and Himelhoch, Taylor, Goldman, and Tandon (1996) versus Tandon et al. (2000).

\section{Psychosis Dimensions}

Four psychosis dimensions (positive, negative, disorganized and depressive) were a priori considered to be of interest. Each had been reliably established as a constellation of symptoms of psychosis in previous work (Dikeos et al., 2006; Grube et al., 1998; Lindenmayer et al., 1995; McGorry et al., 1998). The positive dimension included delusions, ideas of reference, unusual thought content, hallucinations, grandiosity and suspiciousness/persecution. The negative dimension included alogia, affective flattening, avolition, apathy, anhedonia, asociality, social withdrawal, stereotyped thinking and motor retardation. The disorganized dimension included conceptual disorganization, positive formal thought disorder, difficulty in abstract thinking, derailment, tangentiality, incoherence, illogicality, circumstantiality, associative loosening, inattention/distractibility, disorientation, inappropriate affect, bizarre behavior, mannerisms and posturing. The depressive dimension consisted of observed depression, hopelessness, self-depreciation, 
feelings of guilt, guilty ideas of reference, early wakening, suicidal ideation, anxiety and active social avoidance.

The following clinical scales were used in the studies: the Scale for the Assessment of Negative Symptoms (SANS; Andreasen, 1981), the Scale for the Assessment of Positive Symptoms (SAPS; Andreasen, 1984), the Positive and Negative Syndrome Scale (PANSS; Kay, Fiszbein, \& Opler, 1987), Symptoms and Signs of Psychotic Illness (SSPI; Liddle, 1992), the Brief Psychiatry Rating Scale (BPRS; Overall \& Gorham, 1962), Community Adjustment Form (CAF; Test et al., 1991), the High Royds Evaluation of Negativity Scale (HEN; Mortimer, McKenna, Lund, \& Mannuzza, 1989), the Calgary Depression Scale (CDS; D. Addington, Addington, \& Schissel, 1990), the Hamilton Rating Scale for Depression (HRSD; Hamilton, 1960), positive symptoms from the Present State Examination interview (PSE; Wing, Cooper, \& Sartorius, 1974), the Scale for the Assessment of Thought, Language, and Communication (TLC; Andreasen, 1979), the Manchester Scale (Krawiecka, Goldberg, \& Vaughan, 1977), the Positive and Negative and Disorganized Symptoms Scale (PANADSS; Andresen \& Moritz, 2000) and the Comprehensive Assessment of Symptoms and History (CASH; Andreasen, 1987).

\section{Cognitive Domains}

The neuropsychological tests used in the studies were divided into 9 categories that measure approximately the same cognitive construct (Table 1). The classification was based on the MATRICS consensus (Buchanan et al., 2005), which proposed 6 neurocognitive constructs: reasoning and problem solving, speed of processing, attention/vigilance, working memory (verbal), verbal learning and memory, and visual learning and memory. The result of factor analytic studies, these 6 separable neurocognitive factors were replicable across studies and are thought to represent fundamental dimensions of cognitive deficit in schizophrenia (Nuechterlein et al., 2004).

Three other neurocognitive domains were included in the analysis on the basis of evidence of cognitive impairment in specific domains, as reported in previous meta-analyses. IQ was included as a measure of general intelligence (Fioravanti et al., 2005; Heinrichs \& Zakzanis, 1998). In accordance with the discussion in the review by Nuechterlein et al (2004), verbal fluency was examined as a separate factor, given previous meta-analyses that reported substantial impairment of verbal fluency in schizophrenia (Heinrichs \& Zakzanis, 1998; Johnson Selfridge \& Zalewski, 2001). Finally, the construct of executive control was added and assessed with the Stroop Color-Word interference (Stroop, 1935). This test is thought to reflect executive functioning (Derrfuss, 2005), which is substantially impaired in schizophrenia (Johnson Selfridge \& Zalewski, 2001). 
Table 1. Cognitive Domains, Tests and Parameters

\begin{tabular}{|c|c|c|}
\hline Cognitive domain & Cognitive tests & Test parameters \\
\hline IQ & $\begin{array}{l}\text { Wechsler Adult Intelligence Scale and } \\
\text { Revised (Wechsler, 1955,1981) } \\
\text { Wechsler Adult Intelligence Scale- Third } \\
\text { Edition (Wechsler, 1997) }\end{array}$ & Full-scale IQ \\
\hline $\begin{array}{l}\text { Reasoning and } \\
\text { problem solving }\end{array}$ & $\begin{array}{l}\text { Wisconsin Card Sorting Test (Heaton, } \\
\text { 1981) } \\
\text { Nelson's Modified Card Sorting Test } \\
\text { (Nelson, 1976) }\end{array}$ & $\begin{array}{l}\text { Number or percentage of categories } \\
\text { completed } \\
\text { Number or percentage of } \\
\text { perseverative errors }\end{array}$ \\
\hline Executive control & $\begin{array}{l}\text { Stroop Colour-Word Test interference } \\
\text { (Stroop, 1935) }\end{array}$ & $\begin{array}{l}\text { Number of incongruent words read } \\
\text { (number of correct responses) } \\
\text { Number of incongruent errors made }\end{array}$ \\
\hline Verbal fluency & $\begin{array}{l}\text { Either words from a certain category or } \\
\text { words beginning with a certain letter }\end{array}$ & $\begin{array}{l}\text { Number of words generated from } \\
\text { either a certain category or beginning } \\
\text { with a certain letter }\end{array}$ \\
\hline $\begin{array}{l}\text { Speed of } \\
\text { processing }\end{array}$ & $\begin{array}{l}\text { Digit symbol Substitution Test (Wechsler, } \\
\text { 1955) } \\
\text { Trail Making Test Parts A and B (Reitan, } \\
\text { 1958) } \\
\text { Stroop Color-Word Test (Stroop, 1935) }\end{array}$ & $\begin{array}{l}\text { Number of symbols correctly copied } \\
\text { Time in seconds to complete the task } \\
\text { Colors (time in seconds to complete) } \\
\text { Names (time in seconds to complete) }\end{array}$ \\
\hline Attention/vigilance & $\begin{array}{l}\text { Continuous Performance Test } \\
\text { (Nuechterlein \& Dawson, 1984) and its } \\
\text { variations (e.g. Letter-Number Span) }\end{array}$ & $\begin{array}{l}\text { Number or percentage of omissions } \\
\text { Number or percentage of } \\
\text { commissions } \\
\left.\text { Response sensitivity (d'- } A^{\prime}\right)\end{array}$ \\
\hline $\begin{array}{l}\text { Verbal working } \\
\text { memory }\end{array}$ & $\begin{array}{l}\text { Digit Span Backward (Wechsler, 1955) } \\
\text { Letter-Number Span (Gold, 1997) }\end{array}$ & $\begin{array}{l}\text { Total number of digits recalled } \\
\text { Number of correct sequences }\end{array}$ \\
\hline $\begin{array}{l}\text { Verbal learning and } \\
\text { memory }\end{array}$ & $\begin{array}{l}\text { California Verbal Learning Test (Delis, } \\
\text { 1987) } \\
\text { Rey Auditory Verbal Learning Test (Rey, } \\
\text { 1964) } \\
\text { Wechsler Memory Scale-Revised (WMS- } \\
\text { R; Wechsler, 1987), Paired - Associate } \\
\text { Learning subtest } \\
\text { Hopkins Verbal Learning Test (Brandt, } \\
\text { 1991) } \\
\text { Story recall (the Logical Memory subtest } \\
\text { from the WMS-R; Wechsler, 1987) }\end{array}$ & $\begin{array}{l}\text { Total number of correct responses on } \\
\text { either immediate or delayed recall }\end{array}$ \\
\hline $\begin{array}{l}\text { Visual learning and } \\
\text { memory }\end{array}$ & $\begin{array}{l}\text { Benton Visual Retention Test (Benton, } \\
\text { 1992) } \\
\text { WMS-R visual memory (Wechsler, 1987) } \\
\text { Rey-Osterrieth Complex Figure (Rey, } \\
\text { 1941) }\end{array}$ & $\begin{array}{l}\text { Total number of items on either } \\
\text { immediate or delayed recall }\end{array}$ \\
\hline
\end{tabular}


First, data on estimated IQ were reported by 14 studies. Of theses studies, 13 were based on full scale of the Wechsler Adult Intelligence Scale (WAIS; Wechsler, 1955) or the Wechsler Adult Intelligence Scale-Revised (WAIS-R; Wechsler, 1981) and 1 was based on a short form of the Wechsler Adult Intelligence Scale-Third Edition (WAIS-III; Wechsler, 1997). We did not include IQ estimates that were based on 1 or 2 subtests of the WAIS.

Second, reasoning and problem solving was assessed by 30 studies that used the Wisconsin Card Sorting Test (WCST; Heaton, 1981) and 3 studies that used Nelson's Modified Card Sorting Test (Nelson, 1976). Many studies reported two WCST parameters, namely, number of categories achieved and number of perseverative errors; these data were pooled into a combined effect size, as factor analyses have indicated that these variables load on a single factor of perseveration (Nuechterlein et al., 2004).

Third, the construct of executive control was assessed by 10 studies with the Stroop Color-Word interference (Stroop, 1935).

Fourth, verbal fluency, either words from a certain category or words beginning with a certain letter, was assessed in 23 studies.

Fifth, speed of proccessing was assessed using the Digit Symbol Substitution Test (Wechsler, 1955) in 8 studies, the Trail Making Test Parts A and B (Reitan, 1958) in 19 studies, and the Stroop Color-Word name and colour lists (Stroop, 1935) in 3 studies.

Sixth, attention/vigilance was measured by 18 studies that used a Continuous Performance Test (CPT; Nuechterlein \& Dawson, 1984) and its variations. Several studies reported more than one CPT parameter; these were combined into one measure of accuracy. Reaction time variables for the CPT were not used.

Seventh, verbal working memory was assessed with the Digit Span Backward (Wechsler, 1955) in nine studies and with the Letter-Number Span (Gold, Carpenter, Randolph, Goldberg, \& Weinberger, 1997) in two studies.

Eighth, verbal learning and memory was assessed with (a) word list learning tasks (the California Verbal Learning Test, Delis, Kramer, Kaplan, \& Ober, 1987 [5 studies]; the Rey Auditory Verbal Learning Test, Rey, 1964 [4 studies]; the Associate Learning subtest from the Wechsler Memory Scale-Revised [WMS-R], Wechsler, 1987 [5 studies]; or the Hopkins Verbal Learning Test, Brandt, 1991 [3 studies] or (b) story recall (the Logical Memory subtest from the WMS-R, Wechsler, 1987 [8 studies]). Both immediate and delayed recall were included, 
given that the results from factor analysis revealed that both loaded on the same factor (Nuechterlein et al., 2004).

Ninth, visual learning and memory was measured with the Benton Visual Retention Test (Benton, 1992; three studies), the Visual Reproduction subtest from the WMS-R (Wechsler, 1987; five studies), or the Rey Osterrieth Complex Figure (Rey, 1941; six studies); again, immediate and delayed measures were included.

No combined effect sizes were calculated for other possible domains, such as language, reasoning, visuoperceptual and visuospatial functions, or for performance on a single composite cognitive score because of lack of data and/or substantial heterogeneity between the tests used to assess these domains.

\section{Statistical Analysis}

The relevant results from the included studies were quantified in terms of correlations. Because higher scores reflected worse performance for some measures and lower scores reflected worse performance for other measures, all correlations were recoded such that a negative correlation indicated an association between higher levels of symptomatology and worse cognitive performance. Before the meta-analytic methods were applied (as discussed below), the correlations were transformed with Fisher's r-to-z transformation. The results from the meta-analyses were back-transformed into the raw correlation metric whenever possible (e.g., estimated mean correlations, confidence interval bounds). Data extraction and calculations of effect sizes were performed independently by Maria de Gracia Dominguez and Claudia J. P. Simons, who reached consensus in case of discrepancies. All analyses were carried out using the statistical software packages $\mathrm{R}$ and S-plus.

Four steps were performed for the analyses. First, we conducted 36 individual meta-analyses that examined the correlation between the four psychosis dimensions and the nine cognitive domains. Only analyses based on five or more observations were considered. When multiple correlation coefficients were reported for one cognitive domain-psychosis dimension combination within a single sample, these coefficients were first averaged into one $r$ value. We used a random-effects model to account for heterogeneity among the population correlations and to obtain unconditional inferences about the distribution of population correlations (Hedges $\&$ Vevea, 1998). For each of these individual meta-analyses, we report $\mathrm{k}$ (number of studies), $\hat{\mu}_{p}$ (estimated average correlation in the population distribution), 95\% confidence interval $(\mathrm{CI})$ for $\mu_{p}, \mathrm{p}$ ( $\mathrm{p}$ value for the test $H_{0}: \mu_{p}=0$ ) and $\mathrm{I}^{2}$ (percentage of the total variability in the observed correlation coefficients due to 
heterogeneity). Values of $\mathrm{I}^{2}$ equal to 0 indicate the absence of heterogeneity, in which case the random-effects model simplifies to a fixed-effects model. In that case, $\hat{\mu}=\hat{\rho}$, where $\hat{\rho}$ denotes the estimated true (homogeneous) correlation.

As part of this step, we also examined the data for the presence of publication bias. In general, publication bias occurs whenever the results obtained from the published literature are not representative for all of the research that has been conducted on a particular topic. One of the most problematic forms of publication bias is the over- or underrepresentation of particular findings based on their statistical significance. As a result, the estimate of the true correlation can become severely biased. In the absence of this form of publication bias, we would expect to see a more or less symmetric (inverted) funnel when plotted the observed correlations against their corresponding sample sizes (a so-called funnel plot). However, when studies with nonsignificant findings are suppressed from the published literature, the plot can become asymmetrical, and this leads to an association between the correlations and the corresponding sample sizes. The regression test for funnel plot asymmetry examines whether such an association is present in the data; the presence of an association would be indicative of publication bias and would call the findings into doubt (Sterne \& Egger, 2005).

Regression tests for funnel plot asymmetry were conducted for each of the $9 \mathrm{x} 4$ meta-analyses. We also examined funnel plots for asymmetry in terms of the correlations between all of the cognitive domains and each psychopathological dimension (i.e., one funnel plot for each dimension). Because the same sample could contribute multiple correlations to the funnel plot in this case, dependencies are present. We decided not to model these dependencies, as doing so would have required us to obtain information (or estimates) of the $9(8) / 2=36$ intercorrelations between the nine cognitive dimensions. Therefore, the results from the regression test for funnel plot asymmetry for those four funnel plots have to be treated with some caution.

Second, three variables (two sociodemographic and one clinical) were a priori chosen to be included in the analyses as moderators, as these variables have been reported as factors that influence the relationship between cognition and symptoms and therefore may account for at least some of the heterogeneity observed in the meta-analyses. Specifically, the three moderators were (a) chronicity of illness (in years), (b) mean age, and (c) percentage of men. It was hypothesized that studies that included more chronically ill or older or male subjects would show stronger associations between cognition and symptoms (Dikeos et al., 2006; Murray et al., 2005; Schultz et al., 1997; Simonsen et al., 2007). We examined a fourth moderator for the positive dimension that indicated whether the positive subscale included any symptoms of disorganization, as this was done in the older studies in particular. 
Inclusion of symptoms of disorganization in the positive subscale was expected to increase the correlations with the cognitive domains (Liddle \& Morris, 1991; Nieuwenstein et al., 2001).

We used a mixed-effect model was used to examine the influence of the moderators on the average correlation within each psychosis dimension-cognitive domain combination. The amount of residual heterogeneity was estimated with the method of moments estimator based on the basis of weighted least squares (Raudenbush, 1994). Because information about some of the moderator values within the studies was missing, we decided to examine each moderator individually (instead of including all moderators simultaneously within a single model). Results are expressed in terms of the estimated regression coefficient $\hat{\beta}$ and indicate by how much the average correlation (in the transformed units) is estimated to change with a 1 -unit increase in the moderators. For age and chronicity of illness, 1 unit means 1 year; for men, 1 unit means 1\%; and for positive-disorganized, 1 unit means going from (0) positive symptoms to (1) positive and disorganized symptoms. The corresponding $95 \% \mathrm{CI}$ for the true regression coefficient is given also. Because the r-to-z transformation is nonlinear, one cannot easily back-transform the slope of the regression coefficient into the raw correlation metric.

Third, within each of the cognitive domains, we examined whether the average correlation coefficient differed between the negative and disorganized dimensions, in order to further clarify the controversial issue of differential patterns of cognitive impairment related to both (Liddle, 1987; Nieuwenstein et al., 2001). Because numerous samples/studies contributed correlations between a particular domain and both dimensions, we had to account for the dependencies between multiple correlations from a single sample. We estimated the covariance between two r-to-z transformed correlations from a single sample using Equation 10 given by Steiger (1980) and replaced parameters with their corresponding sample estimates where necessary. In doing so, we had to estimate the correlation between the two dimensions on the basis on the previous literature (none of the studies reported these correlations). For this analysis, the correlation between the negative and the disorganized dimension was estimated at .39 (Peralta, Cuesta, \& de Leon, 1994). Sensitivity analyses were conducted using two other assumed correlation values (i.e., .20 and .60).

Fourth, in order to deal with potential overlap in the samples in the three previous steps of the statistical analyses, we conducted sensitivity analyses that excluded the smallest studies of each pair with an uncertain degree of overlap, as discussed before. 


\section{Results}

Of the 3129 studies produced by the search, 187 were considered eligible for inclusion. Of these, 129 were excluded, because (a) the study examined associations with single symptom items ( $3.1 \%$ of the total number of studies not included); (b) the study split the subject sample into groups on the basis of their score on the different clinical scales $(27.9 \%)$ or on the basis of their cognitive performance (6.2\%) and reported the associations with these groups; (c) the study reported only correlations that were statistically significant (24\%); (d) the study used neuropsychological tasks that were not used in any of the other included studies or that could not be classified under one of the cognitive domains (24\%); (e) the study reported global cognitive or psychopathology scores (2.3\%); (f) the study sample completely overlapped with a larger study that was included $(2.3 \%)$; or $(\mathrm{g})$ the authors did not reply to the request to provide additional data (10.1\%).

The literature search thus yielded 58 studies that evaluated psychosis dimensions and cognitive performance in patients with a lifetime history of nonaffective psychosis, according to standardized neuropsychological instruments. These studies are listed in Table 2, along with the sample sizes and the main sample characteristics. In total, 5,009 individuals with a diagnosis of non-affective psychosis contributed to this meta-analysis. The patients were $72.7 \%$ men (reported by 57 articles). The mean age of the patients ranged from 19.1 to 51.9 years (reported by 55 articles). Mean chronicity of illness ranged from 4.8 to 28.9 years (reported by 37 articles). The mean age of illness onset ranged from 18.6 to 26.23 years (reported by 18 articles). The mean years of education ranged from 9.9 to 13.7 (reported by 39 articles). A total of $58 \%$ of the subjects were inpatients (reported by 44 articles). Of the patients, $94 \%$ were diagnosed with schizophrenia, 3\% with schizoaffective disorder, and 3\% with delusional and other psychotic disorders (reported by 55 articles). Other variables, such as level of symptoms, dosage of antipsychotic medication, percentage of patients on typical versus atypical medication, and number of prior psychotic episodes, may be relevant for the association between symptoms and cognition but were reported by too few studies. A table with all data points is not reported due to space constraints. 


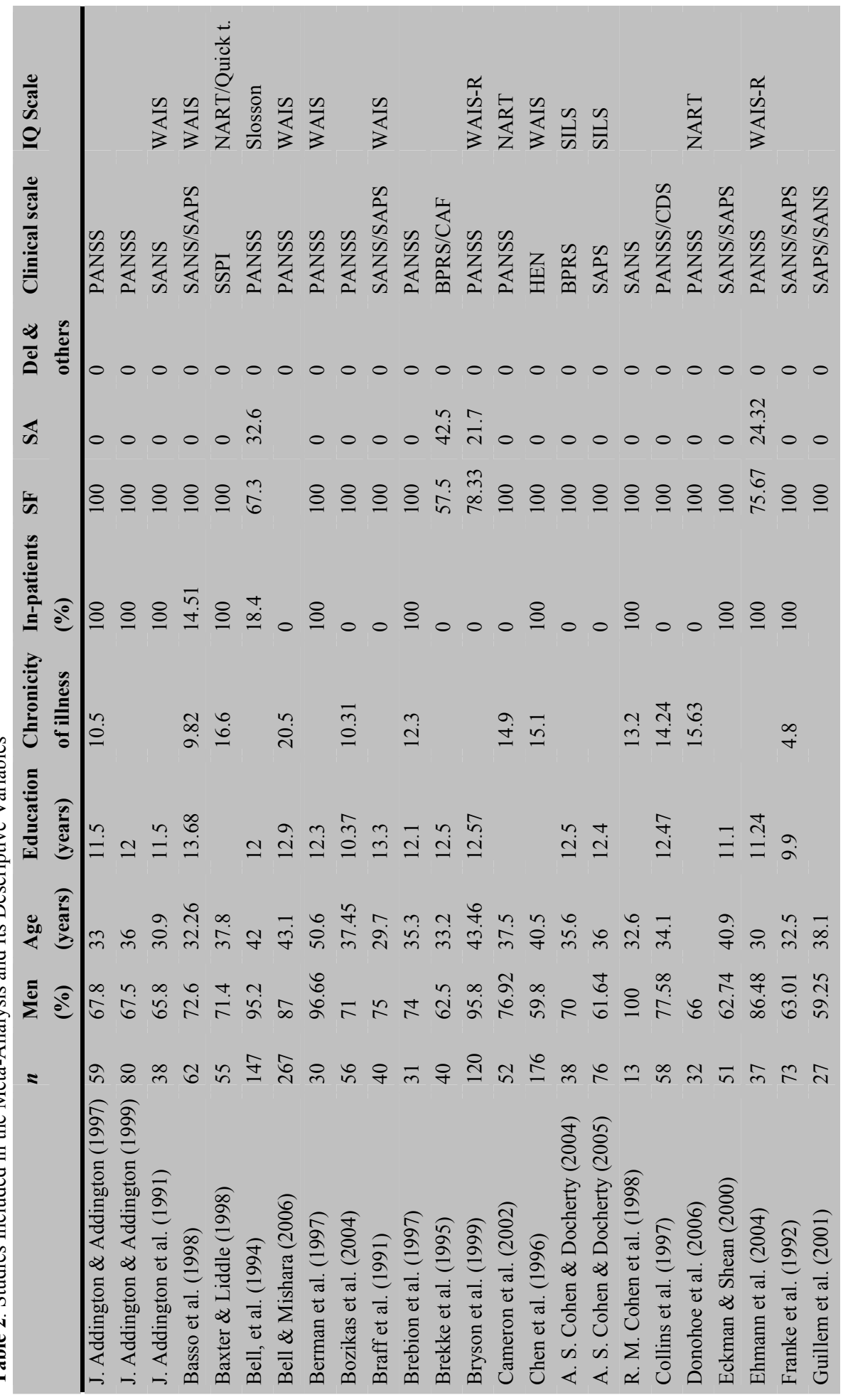




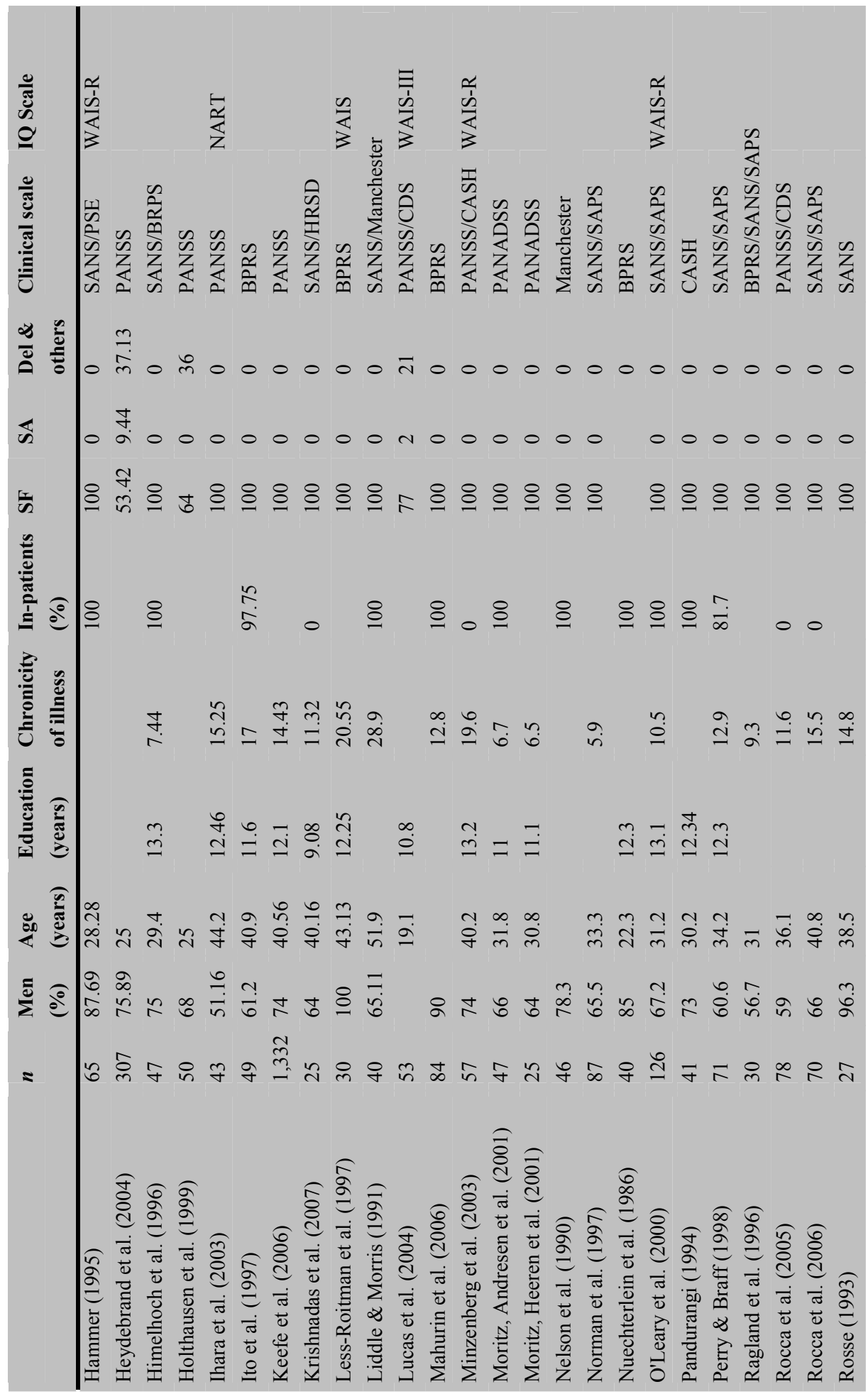




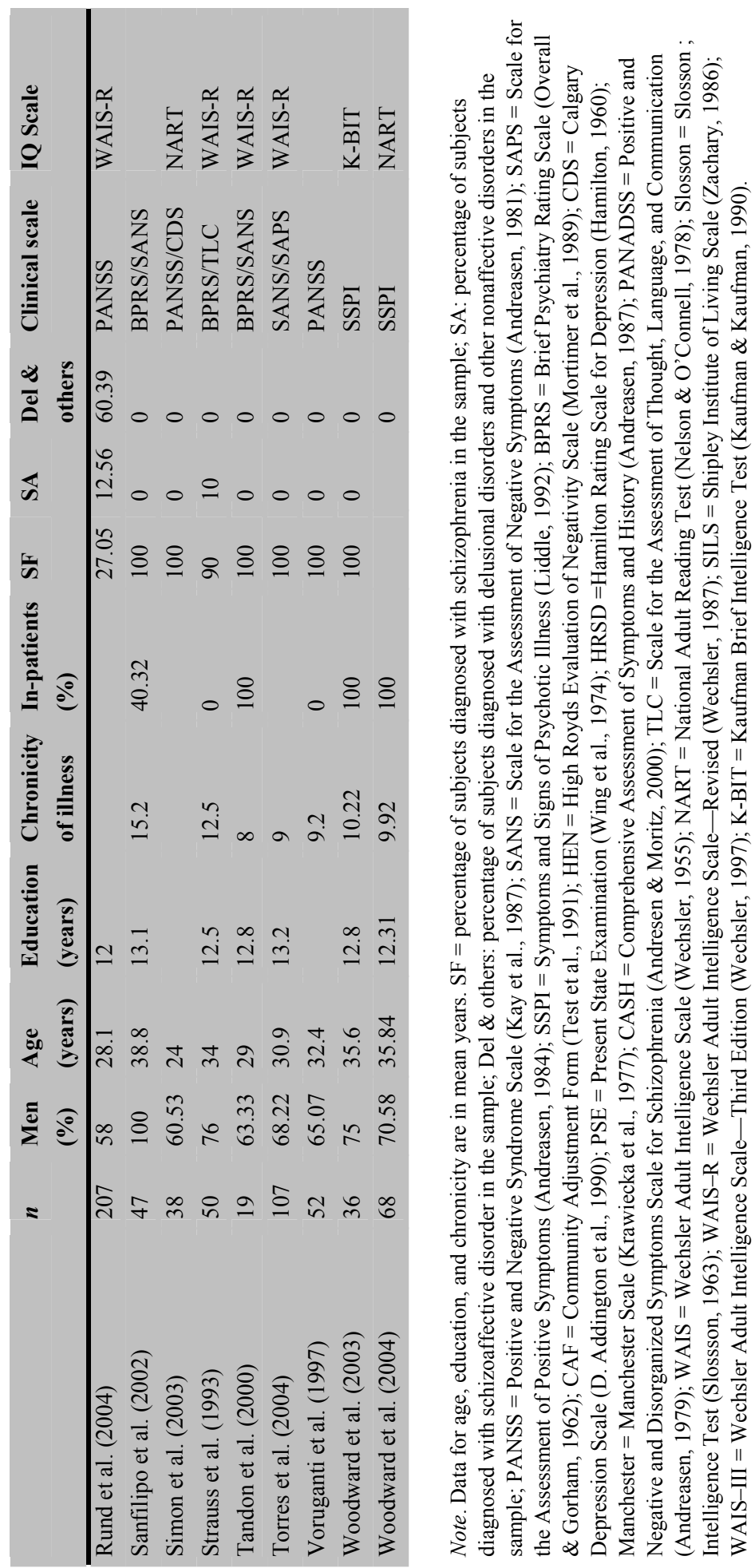




\section{Meta-analyses of Correlations between Cognitive Domains and Psychosis Dimensions}

Results for the meta-analyses are shown in Table 3 (for visual representation, see Figure 1). Both the negative and the disorganized dimensions were significantly and negatively correlated with the majority of the nine cognitive domains, with averaged correlations in the order of -.29 to -.12 . The largest effect sizes (estimated correlation higher than -.2) were for verbal fluency, verbal learning and memory, and IQ in the negative dimension and for attention/vigilance, visual learning and memory, and IQ in the disorganized domain. The correlations with the positive dimension were neither large nor significant, except for a negative correlation with the domain of speed of processing $\left(\hat{\mu}_{p}=-.089 ; 95 \% \mathrm{CI}=-.164,-.012\right)$, which indicated that worse performance was associated with a higher level of symptoms. For the analysis of the depressive dimension, none of the correlations were either large or significant; however, five or more observations were available for only three cognitive domains.

Figure 1. Meta-analyses of Correlation Coefficients between Four Psychosis Dimensions and Nine Neurocognitive Domains in Nonaffective psychosis.



Note: Boldface values indicate associations between psychosis dimensions and neurocognitive domains for which the correlation was statistically significant. CI $=95 \%$ confidence interval. 
Table 3. Four $x$ Nine Meta-analyses of Correlation Coefficients Between Psychosis Dimensions and Neurocognitive Domains

\begin{tabular}{|c|c|c|c|c|c|}
\hline Cognitive domain & & Positive & Negative & Disorganized & Depressive \\
\hline IQ & $\mathrm{k}$ & 10 & 13 & 6 & 1 \\
\hline & $\hat{\mu}_{\rho}$ & 0.024 & -0.244 & -0.205 & \\
\hline & $\mathrm{CI}$ & $-0.063,0.111$ & $-0.333,-0.151$ & $-0.327,-0.076$ & \\
\hline & $\mathrm{p}$ & 0.591 & $\mathbf{0}$ & 0.002 & \\
\hline & $\mathrm{I}^{2}$ & 26 & 52 & 45 & \\
\hline Reasoning and problem solving & $\mathrm{k}$ & 27 & 33 & 15 & 6 \\
\hline & $\hat{\mu}_{\rho}$ & -0.013 & -0.14 & -0.197 & 0.074 \\
\hline & $\mathrm{CI}$ & $-0.066,0.041$ & $-0.197,-0.081$ & $-0.336,-0.048$ & $-0.024,0.171$ \\
\hline & $\mathrm{p}$ & 0.639 & $\mathbf{0}$ & 0.009 & 0.137 \\
\hline & $\mathrm{I}^{2}$ & 37 & 58 & 81 & 0 \\
\hline Executive control & $\mathrm{k}$ & 9 & 10 & 7 & 3 \\
\hline & $\hat{\mu}_{\rho}$ & 0.082 & -0.131 & -0.089 & 0.059 \\
\hline & CI & $-0.017,0.179$ & $-0.265,0.008$ & $-0.202,-0.026$ & $-0.264,0.369$ \\
\hline & $\mathrm{p}$ & 0.103 & 0.063 & 0.130 & 0.725 \\
\hline & $\mathrm{I}^{2}$ & 6 & 56 & 20 & 73 \\
\hline Verbal fluency & $\mathrm{k}$ & 20 & 23 & 13 & 4 \\
\hline & $\hat{\mu}_{\rho}$ & -0.035 & -0.291 & -0.092 & -0.056 \\
\hline & CI & $-0.101,0.031$ & $-0.356,-0.224$ & $-0.208,0.027$ & $-0.199,0.089$ \\
\hline & $\mathrm{p}$ & 0.292 & $\mathbf{0}$ & 0.128 & 0.446 \\
\hline & $\mathrm{I}^{2}$ & 17 & 42 & 61 & 0 \\
\hline Speed of processing & $\mathrm{k}$ & 20 & 23 & 13 & 5 \\
\hline & $\hat{\mu}_{\rho}$ & -0.089 & -0.167 & -0.171 & -0.097 \\
\hline & CI & $-0.164,-0.012$ & $-0.241,-0.09$ & $-0.275,-0.062$ & $-0.256,0.068$ \\
\hline & $\mathrm{p}$ & 0.023 & $\mathbf{0}$ & 0.002 & 0.250 \\
\hline & $\mathrm{I}^{2}$ & 46 & 53 & 56 & 46 \\
\hline Attention/vigilance & $\mathrm{k}$ & 11 & 15 & 6 & 2 \\
\hline & $\hat{\mu}_{\rho}$ & -0.012 & -0.134 & -0.277 & 0.154 \\
\hline & $\mathrm{CI}$ & $-0.054,0.03$ & $-0.191,-0.076$ & $-0.392,-0.154$ & $-0.023,0.257$ \\
\hline & $\mathrm{p}$ & 0.969 & $\mathbf{0}$ & $\mathbf{0}$ & 0.087 \\
\hline & $\mathrm{I}^{2}$ & 0 & 26 & 34 & 0 \\
\hline Verbal working memory & $\mathrm{k}$ & 9 & 10 & 5 & 3 \\
\hline & $\hat{\mu}_{\rho}$ & -0.013 & -0.07 & -0.117 & -0.113 \\
\hline & $\mathrm{CI}$ & $-0.144,0.118$ & $-0.174,0.036$ & $-0.247,0.018$ & $-0.303,0.085$ \\
\hline & $\mathrm{p}$ & 0.843 & 0.194 & 0.09 & 0.263 \\
\hline & $\mathrm{I}^{2}$ & 37 & 19 & 0 & 5 \\
\hline Verbal learning \& memory & $\mathrm{k}$ & 17 & 20 & 13 & 3 \\
\hline & $\hat{\mu}_{\rho}$ & -0.021 & -0.214 & -0.169 & 0.126 \\
\hline & $\mathrm{CI}$ & $-0.096,0.054$ & $-0.279,-0.146$ & $-0.27,-0.064$ & $-0.035,0.281$ \\
\hline & $\mathrm{p}$ & 0.578 & $\mathbf{0}$ & 0.001 & 0.123 \\
\hline & $\mathrm{I}^{2}$ & 47 & 54 & 59 & 0 \\
\hline Visual learning \& memory & $\mathrm{k}$ & 9 & 13 & 6 & 3 \\
\hline & $\hat{\mu}_{\rho}$ & -0.005 & -0.126 & -0.206 & 0.029 \\
\hline & $\mathrm{CI}$ & $-0.089,0.079$ & $-0.202,-0.047$ & $-0.331,-0.074$ & $-0.147,0.203$ \\
\hline & $\mathrm{p}$ & 0.91 & 0.001 & 0.002 & 0.749 \\
\hline & $\mathrm{I}^{2}$ & 0 & 29 & & \\
\hline
\end{tabular}

Note: Boldface values indicate associations between psychosis dimensions and neurocognitive domains for which the correlation was statistically significant. $\mathrm{CI}=95 \%$ confidence interval. 


\section{CHAPTER 2}

\section{Regression Test for Funnel Plot Asymmetry}

None of the regression tests for funnel plot asymmetry were significant for the 36 pairs of psychosis dimensions and cognitive domains, except for the combination of depressive dimension and visual learning and memory $(\mathrm{p}=.014)$. However, only three observations were included in this analysis, so this result should be treated with some reservation. The funnel plots for each symptom dimension combine all cognitive domains are shown in Figure 2. None of the regression tests for asymmetry were significant for these four plots ( $\mathrm{ps}=.145-.927)$.

Figure 2. Regression Test for Funnel Plot Asymmetry

for the Associations of Cognitive Domains within each Psychopathological Dimension.

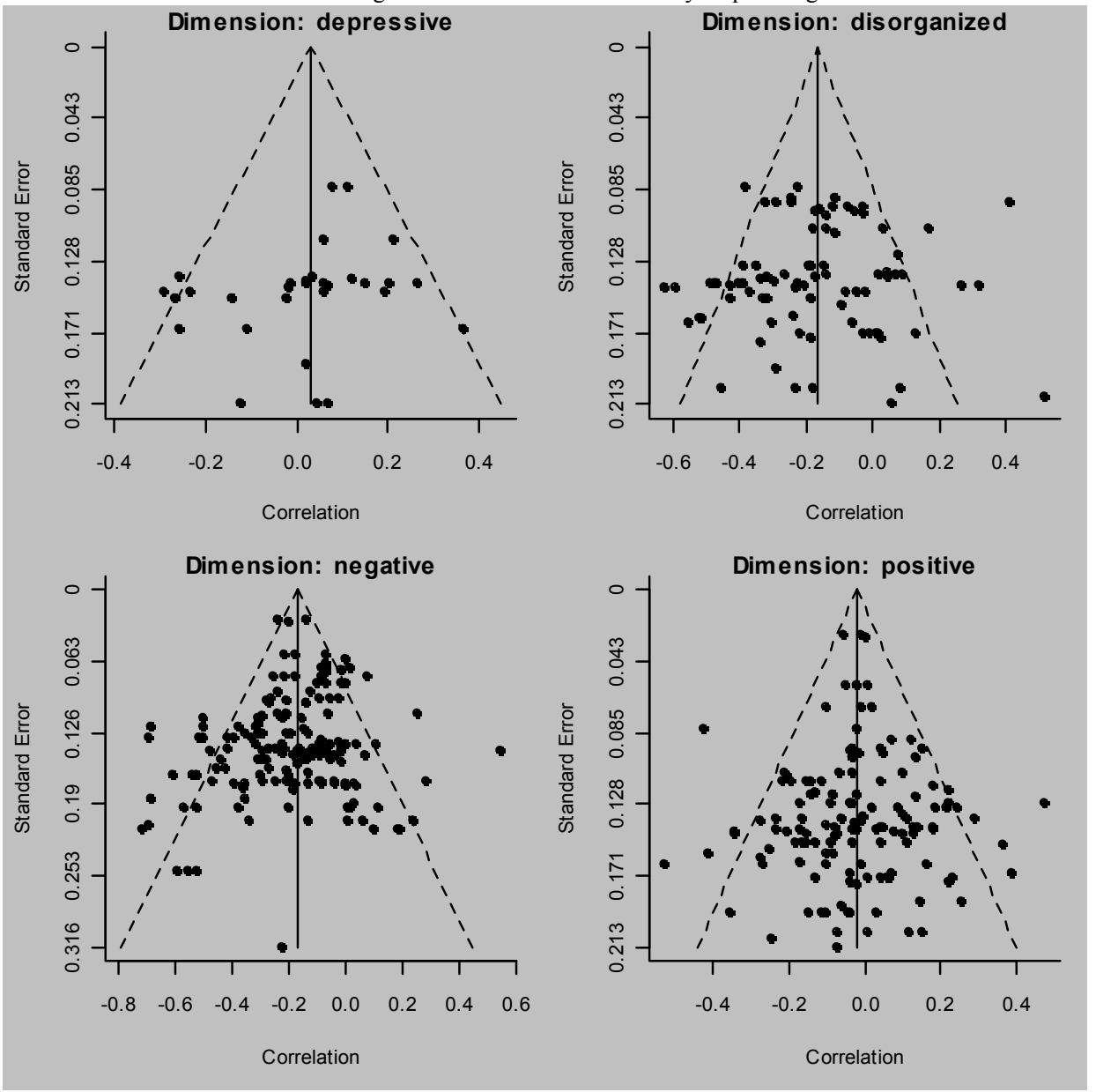




\section{Effect of Moderator Variables}

Substantial heterogeneity was found in the correlations between psychosis dimensions and cognitive domains. However, none of the three variables included as possible moderators could account for the observed heterogeneity. The effect of gender on the average correlation was not significant for any of the meta-analyses (all $\hat{\beta}$ values between -.037 and .017, all ps between .054 and .996). Likewise, age did not influence the strength of the associations between the cognitive domains and symptom dimensions (all $\hat{\beta}$ values between -.288 and .020 , all ps between .127 and .983 ), except for the association of the depressive dimension and speed of processing $(\hat{\beta}=.020$, ps $=.047$ ), the strength of which became weaker with increases in the average age of the sample. Heterogeneity in the correlations between cognitive domains and symptom dimensions could not be attributed to differences in the chronicity of the illness (all $\hat{\beta}$ values between -.049 and .150 , all ps between .055 and .977 ), except for the association of the disorganized dimension and IQ $(\hat{\beta}=.037, \mathrm{ps}=.022)$, the strength of which became weaker with increases in the average duration of the illness. However, these two findings are inconsistent with the general pattern of results and may constitute Type I errors. Finally, inclusion of symptoms of disorganization in the positive subscale led to a stronger correlation only with respect to IQ ( $\hat{\beta}=-.157, \mathrm{ps}=.033$; all other $\hat{\beta}$ values between -.134 and .110 , all other ps between .131 and .849). Again, this isolated result is not consistent with the findings for the other eight cognitive domains.

\section{Differential Correlation Coefficients for Cognitive Impairment: Negative Versus. Disorganized Dimension}

The negative dimension showed a significantly stronger correlation with verbal fluency $(\mathrm{p}=.005)$, whereas the disorganized dimension showed stronger correlations with reasoning and problem solving $(\mathrm{p}=.004)$ and attention/vigilance $(\mathrm{p}=.03)$. The correlation coefficients did not differ significantly between the two dimensions for the other six cognitive domains. The sensitivity analysis (i.e., assuming different values for the strength of the association between negative and disorganized symptoms) corroborated these results.

\section{Sensitivity Analyses to Account for Sample Overlap}

The sensitivity analysis (i.e., those excluding the smaller studies of each pair with an uncertain degree of overlap in the samples) corroborated all of the results in the three previous steps of the analyses. 


\section{Discussion}

\section{Findings}

Psychopathological heterogeneity in nonaffective psychosis was weakly but differentially related to distinct patterns of neurocognitive function (Figure 1): negative and disorganized dimensions were modestly but meaningfully associated with impairment in the majority of neurocognitive domains. Associations were in the expected direction but smaller and nonsignificant for the domains of executive control and verbal working memory for both dimensions and for verbal fluency in relation to the disorganized dimension. Thus, cognitive impairment in psychosis (Aleman, Hijman, de Haan, \& Kahn, 1999; Fioravanti et al., 2005; Heinrichs \& Zakzanis, 1998; Johnson Selfridge \& Zalewski, 2001; Lee \& Park, 2005; Pelletier, Achim, Montoya, \& Lal, \& Lepage, 2005) does not appear to be entirely orthogonal to psychopathology. In addition, significantly higher correlations were found for the negative dimension in relation to verbal fluency and for the disorganized dimension in relation to reasoning and problem solving and to attention/vigilance. In contrast, positive and depressive dimensions were not consistently associated with the neurocognitive measures examined, with the exception of a significant correlation found for the positive dimension in relation to speed of processing.

The patterns of association for the four psychosis dimensions were stable across neurocognitive domains and were independent of age, gender and chronicity of illness. These findings in relation to the negative and positive symptoms agree with a more limited previous meta-analysis (Nieuwenstein et al., 2001), whereas the findings with regard to the disorganized dimension were in agreement with a metaanalysis that focused on formal thought disorder (Kerns \& Berenbaum, 2002). The results further suggest that distinguishing multiple domains of cognitive functioning in schizophrenia is useful, even though the deficit is to a large extent generalized and may even be accounted for statistically by a single-factor model (as suggested by the CATIE trial, Keefe et al, 2006). Differential patterns of cognitive performance among the different psychopathological dimensions were found. These patterns may indicate some meaningful contrasts suggestive of differential latent cerebral mechanisms underlying the cluster of disorganized and negative symptoms on the one hand and that of positive and affective on the other.

\section{Do Patterns of Psychopathology-Neurocognition Associations in Nonaffective Psychosis Match Evidence of Differential Underlying Patterns of Cerebral Dysfunction?}

Functional brain imaging studies have demonstrated that different patterns of altered cerebral activity, possibly reflective of clinical heterogeneity, occur in schizophrenia. Early studies (Ebmeier et al., 1993; Heaton, 1985; Liddle, Friston, 
Frith, \& Frackowiak, 1992; Liddle, Friston, Frith, Hirsch et al., 1992; Nelson, 1976; Weinberger \& Berman, 1988; Weinberger, Berman, \& Illowsky, 1988; Weinberger, Berman, \& Zec, 1986) have tended to concur on findings suggesting that (a) psychomotor poverty is associated with prefrontal cortex and parietal cortex underactivity, especially on the left, and with overactivity of the caudate nuclei bilaterally; (b) the disorganization syndrome is associated with decreased perfusion in the right ventral prefrontal cortex and contiguous insula and with increased perfusion in the right anterior cingulated; and (c) reality distortion is associated with overactivity in the left medial temporal lobe and left lateral frontal lobe and underactivity in the left lateral temporal lobe, adjacent parietal cortex, and posterior cingulated cortex. Later brain imaging studies in schizophrenia (Lawrie \& Abukmeil, 1998; Lieberman et al., 2001) confirmed that negative (Andreasen, Paradiso, \& O'Leary, 1998; Sanfilipo et al., 2000; Schroder et al., 1996) and disorganization symptoms (Crider, 1997; Liddle, Friston, Frith, Hirsch et al., 1992) are associated with alterations in frontal lobe functioning, whereas auditory hallucinations produced normal activation of the left prefrontal cortex but exhibited less activation of the left middle temporal gyrus and supplementary motor area (Lennox, Park, Medley, Morris, \& Jones, 2000; McGuire et al., 1995; Shergill, Brammer, Williams, Murray, \& McGuire, 2000; Shergill, Bullmore, Simmons, Murray, \& McGuire, 2000). Recently, abnormal connectivity has been postulated as the central functional cerebral abnormality in schizophrenia (Josin \& Liddle, 2001; Menon, Anagnoson, Glover, \& Pfefferbaum, 2001). This possibility would indicate that the core abnormality may be a disruption of functional connectivity between frontal cortex and other cerebral sites that gives rise to distributed cortical and subcortical deficits that may be associated with differential behavioural expression, such as variation in symptom dimensions and cognition.

At the level of neurotransmission, different patterns involving several interconnected limbic, cortical and subcortical structures have been implicated in schizophrenia. The dopamine (DA) hypothesis has been extended to include both cortical and subcortical components. An overactivity in neurotransmission from DA cell bodies (D2 receptors), located in the ventral tegmental area of the midbrain, may result in the development of positive symptoms. A hypodopaminergic state in the prefrontal cortical terminal fields of the mesocortical DA neurons (D1 receptors) has been hypothesized to underly cognitive impairment and negative symptoms of schizophrenia and may in turn contribute to the disinhibition of subcortical DA function (Duncan, Sheitman, \& Lieberman, 1999; Kellendonk et al., 2006). Moreover, alterations in prefrontal connectivity involving glutamate transmission at N-methyl-D-aspartate (NMDA) receptors has been proposed. This hypothesis suggests that the DA imbalance in schizophrenia (striatal excess and cortical deficiency) may be secondary to NMDA hypofunction in the prefrontal cortex and its connections (Laruelle, Kegeles, \& Abi-Dargham, 2003). 


\section{Negative and Disorganized Dimensions Versus Positive and Affective Dimensions: Does Clinical Research Suggest Two Pathways in Psychosis?}

There is evidence that intermediary phenotypes associated with genetic risk of psychosis may fall into two broad groups: one associated with cognitive impairment and negative and disorganized symptom dimensions and one associated with altered sensitivity to stress expressed as increased levels of affective and positive psychotic symptoms following exposure to small stressors in the flow of daily life (MyinGermeys \& van Os, 2007). Thus, increased emotional reactivity correlates negatively with cognitive impairment in patients with schizophrenia (Myin Germeys et al., 2002). The two intermediary phenotypes of stress sensitivity and cognitive impairments may therefore constitute separate and even mutually exclusive pathways to psychosis associated with partly different expression of psychopathology.

Another area of research that may help understand the pathways of differential expression of psychopathology is social cognition (Blakemore \& Frith, 2004; Freeman, Garety, \& Kuipers, 2001; Frith, 2004; Kuipers et al., 2006). Positive psychotic symptomatology has been associated with impaired social cognition, such as alterations in self-monitoring (Bentall, 1990; Johns et al., 2001), a probabilistic reasoning bias referred to as jumping-to-conclusions (Garety \& Freeman, 1999; Garety, Kuipers, Fowler, Freeman, \& Bebbington, 2001; van Dael et al., 2006), and mentalizing deficits (Versmissen et al., 2007). Where examined, research suggests that the association between altered social cognition and symptoms is weakest for the negative and disorganization dimensions (van Dael et al., 2006). These observations tend to agree with our finding that positive and affective symptoms were not associated with neurocognition, whereas negative and disorganized dimensions were. Recent analyses support this view and show that, in patient samples, alterations in social cognition are largely independent of measures of neurocognition (van Hooren et al., submitted).

\section{Conclusion: Different Underlying Pathophysiological Processes Associated with Different Intermediary Phenotypes May Account for a Substantial Part of Psychopathological Heterogeneity in Nonaffective Psychosis}

Findings at different levels of neuroscientific and neurocognitive research have suggested some evidence of meaningful contrasts in the underlying patterns of cerebral dysfunction in relation to the psychopathological heterogeneity of psychosis. Neuroimaging, neuropharmacological and neurocognitive findings concur in that weak but systematic patterns of associations are found with dimensions of psychopathology. These associations fit in the simplistic but heuristically useful two-pathway model of psychosis, in which the exophenotypes of negative and disorganized symptoms are associated with the intermediary 
phenotype of neurocognitive impairment and the positive and affective dimensions are not. This reduction may explain at least some of the psychopathological heterogeneity in nonaffective psychosis and may be refined in future studies, to the extent that they become nosologically useful.

\section{Methodological issues}

First, four rather than five psychosis dimensions were analyzed, although evidence suggests that a five-factor solution that includes a manic symptom dimension may yield a better fit (Dikeos et al., 2006; Grube et al., 1998; Lindenmayer et al., 1995; McGorry et al., 1998; Serretti, De Ronchi, Lorenzi, \& Berardi, 2004; Serretti et al., 2001). However, because only four studies reported the manic/excitement dimension, there were too few data for an informative synthesis. Second, only nonaffective psychotic disorder was considered in the inclusion criteria for study selection. The reason for this was that although rather similar patterns of cognitive impairment have been found in the affective domain of psychosis (Fioravanti et al., 2005; Krabbendam, Arts, van Os, \& Aleman, 2005) and rather similar psychopathological dimensions have been identified in affective and nonaffective psychosis (Peralta et al, Biol Psychiatry, 1997), associations between psychopathological dimensions and neurocognition have been examined to only a substantial degree in nonaffective psychotic disorders. Third, some of the included cognitive tests may vary in terms of sensitivity, and this variance may be problematic in view of the generalized cognitive deficit in schizophrenia (Chapman \& Chapman, 1978). That is, the difference between performance of patients with schizophrenia and healthy controls will be greater for tasks with higher reliability and variance, regardless of differences in true ability. Such variation may result in different likelihoods of correlating with other parameters, such as symptom dimensions. This problem can be solved only by using tasks that are matched on the relevant psychometric characteristics. This is a limitation that should be acknowledged by each systematic review that summarizes and combines different cognitive tests. Fourth, because all subjects in the selected studies were younger than 51.9 years of age, no conclusions can be made regarding the impact of aging for each psychopathology dimension-cognition combination. Fifth, many other moderator variables may be of relevance; however, due to underreporting, they could not be examined in more detail (e.g., clinical scales scores, pharmacological treatment, previous history of symptoms, genetic vulnerability, hemisphere correlation, and comorbidity). Sixth, exclusion of a number of studies was necessary because of incomplete information, resulting in sample restriction. 


\section{Author note}

Preparation of this article was supported by grants and financial support: Maria de Gracia Dominguez, Marie Curie Early Stage Research Training Fellowship of the European Community's Sixth Framework Program under contract number "MESTCT-2005-020589"; Jim van Os, research support by Eli Lilly, GSK, Organon, BMS, AstraZeneca, and speaker support by Eli Lilly, BMS, Lundbeck, Organon, JanssenCilag and AstraZeneca; Lydia Krabbendam, VIDI-grant from the Netherlands Organization for Scientific Research (NWO).

\section{References}

References marked with an asterisk indicate studies included in the meta-analysis.

Addington, D., Addington, J., \& Schissel, B. (1990). A depression rating scale for schizophrenics. Schizophrenia Research, 3(4), 247-251.

*Addington, J., \& Addington, D. (1997). Attentional vulnerability indicators in schizophrenia and bipolar disorder. Schizophrenia Research, 23(3), 197-204.

Addington, J., \& Addington, D. (1998). Facial affect recognition and information processing in schizophrenia and bipolar disorder. Schizophrenia Research, 32(3), 171-181.

*Addington, J., \& Addington, D. (1999). Neurocognitive and social functioning in schizophrenia. Schizophrenia Bulletin, 25(1), 173-182.

*Addington, J., Addington, D., \& Maticka Tyndale, E. (1991). Cognitive functioning and positive and negative symptoms in schizophrenia. Schizophrenia Research, 5(2), 123-134.

Aleman, A., Hijman, R., de Haan, E. H., \& Kahn, R. S. (1999). Memory impairment in schizophrenia: A meta-analysis. American Journal of Psychiatry, 156(9), 1358-1366.

American Psychiatric Association. (1980). Diagnostic and statistical manual of mental disorders (DSM-III) (3nd edn.). Washington, DC: American Psychiatric Association.

American Psychiatric Association. (1987). Diagnostic and statistical manual of mental disorders (DSM-IIIR) (3nd edn. revised). Washington, DC: American Psychiatric Association.

American Psychiatric Association. (1994). Diagnostic and statistical manual of mental disorders (DSM-IV) (4nd ed.). Washington, DC: American Psychiatric Association.

Ammons, R. B., \& Ammons, C. H. (1962). The Quick Test (QT): Provisional manual. Psychological Reports, $11,111-161$.

Andreasen, N. C. (1979). Thought, language, and communication disorders. I. Clinical assessment, definition of terms, and evaluation of their reliability. Archives of General Psychiatry, 36(12), 1315-1321.

Andreasen, N. C. (1981). Scale for the assessment of negative symptoms (SANS). The University of Iowa, Iowa City. IA.

Andreasen, N. C. (1984). The scale for the assessment of positive symptoms (SAPS). Iowa City: The University of Iowa.

Andreasen, N. C. (1987). Comprehensive assessment of symptoms and history. Iowa City: University of Iowa, College of Medicine.

Andreasen, N. C., Paradiso, S., \& O'Leary, D. S. (1998). "Cognitive dysmetria" as an integrative theory of schizophrenia: A dysfunction in cortical-subcortical-cerebellar circuitry? Schizophrenia Bulletin, 24(2), 203-218.

Andresen, B., \& Moritz, S. (2000). Positive and negative and disorganized symptoms scale for schizophrenia (PANADSS). Manual. Westerau, Germany: PPV.

*Basso, M. R., Nasrallah, H. A., Olson, S. C., \& Bornstein, R. A. (1998). Neuropsychological correlates of negative, disorganized and psychotic symptoms in schizophrenia. Schizophrenia Research, 31(2-3), 99111.

*Baxter, R. D., \& Liddle, P. F. (1998). Neuropsychological deficits associated with schizophrenic syndromes. Schizophrenia Research, 30(3), 239-249. 
*Bell, M. D., Lysaker, P. H., Milstein, R. M., \& Beam Goulet, J. L. (1994). Concurrent validity of the cognitive component of schizophrenia: Relationship of PANSS scores to neuropsychological assessments. Psychiatry Research, 54(1), 51-58.

*Bell, M. D., \& Mishara, A. L. (2006). Does negative symptom change relate to neurocognitive change in schizophrenia? Implications for targeted treatments. Schizophrenia Research, 81(1), 17-27.

Bentall, R. P. (1990). The illusion of reality: A review and integration of psychological research on hallucinations. Psychological Bulletin, 107(1), 82-95.

Benton, S. A. (1992). Benton visual retention test manual (5th ed.). San Antonio, TX: Harcourt Brace \& Company.

*Berman, I., Viegner, B., Merson, A., Allan, E., Pappas, D., \& Green, A. I. (1997). Differential relationships between positive and negative symptoms and neuropsychological deficits in schizophrenia. Schizophrenia Research, 25(1), 1-10.

Blakemore, S. J., \& Frith, U. (2004). How does the brain deal with the social world? Neuroreport, 15(1), 119128.

*Bozikas, V. P., Kosmidis, M. H., Kioperlidou, K., \& Karavatos, A. (2004). Relationship between psychopathology and cognitive functioning in schizophrenia. Comprehensive Psychiatry, 45(5), 392400.

*Braff, D. L., Heaton, R., Kuck, J., Cullum, M., Moranville, J., Grant, I., et al. (1991). The generalized pattern of neuropsychological deficits in outpatients with chronic schizophrenia with heterogeneous Wisconsin card sorting test results. Archives of General Psychiatry, 48(10), 891-898.

Brandt, J. (1991). The Hopkins verbal learning test: Development of a new memory test with six equivalent forms. Clinical Neuropsychology, 5, 125-142.

*Brebion, G., Smith, M. J., Amador, X., Malaspina, D., \& Gorman, J. M. (1997). Clinical correlates of memory in schizophrenia: Differential links between depression, positive and negative symptoms, and two types of memory impairment. American Journal of Psychiatry, 154(11), 1538-1543.

*Brekke, J. S., Raine, A., \& Thomson, C. (1995). Cognitive and psychophysiological correlates of positive, negative, and disorganized symptoms in the schizophrenia spectrum. Psychiatry Research, 57(3), 241250 .

*Bryson, G., Bell, M., Greig, T., \& Kaplan, E. (1999). Internal consistency, temporal stability and neuropsychological correlates of three cognitive components of the positive and negative syndrome scale (PANSS). Schizophrenia Research, 38(1), 27-35.

Buchanan, R. W., Davis, M., Goff, D., Green, M. F., Keefe, R. S., Leon, A. C., et al. (2005). A summary of the FDA-NIMH-MATRICS workshop on clinical trial design for neurocognitive drugs for schizophrenia. Schizophrenia Bulletin, 31(1), 5-19.

*Cameron, A. M., Oram, J., Geffen, G. M., Kavanagh, D. J., McGrath, J. J., \& Geffen, L. B. (2002). Working memory correlates of three symptom clusters in schizophrenia. Psychiatry Research, 110(1), 49-61.

Chapman, L., \& Chapman, J. (1978). The measurement of differential deficit. Journal of Psychiatric Research, 14, 303-311.

*Chen, E. Y., Lam, L. C., Chen, R. Y., \& Nguyen, D. G. (1996). Negative symptoms, neurological signs and neuropsychological impairments in 204 Hong Kong Chinese patients with schizophrenia. The British Journal of Psychiatry, 168(2), 227-233.

*Cohen, A. S., \& Docherty, N. M. (2004). Deficit versus negative syndrome in schizophrenia: Prediction of attentional impairment. Schizophrenia Bulletin, 30(4), 827-835.

*Cohen, A. S., \& Docherty, N. M. (2005). Symptom-oriented versus syndrome approaches to resolving heterogeneity of neuropsychological functioning in schizophrenia. The Journal of Neuropsychiatry and Clinical Neurosciences, 17(3), 384-390.

*Cohen, R. M., Nordahl, T. E., Semple, W. E., Andreason, P., \& Pickar, D. (1998). Abnormalities in the distributed network of sustained attention predict neuroleptic treatment response in schizophrenia. Neuropsychopharmacology, 19(1), 36-47.

*Collins, A. A., Remington, G. J., Coulter, K., \& Birkett, K. (1997). Insight, neurocognitive function and symptom clusters in chronic schizophrenia. Schizophrenia Research, 27(1), 37-44.

Crider, A. (1997). Perseveration in schizophrenia. Schizophrenia Bulletin, 23(1), 63-74.

Delis, D. C., Kramer, J. H., Kaplan, E., \& Ober, B. A. (1987). California verbal learning test: Adult version. San Antonio, TX: The Psychological Corporation. 
Derrfuss, J., Brass, M., Neumann, J., \& von Cramon, D. Y. (2005). Involvement of the inferior frontal junction in cognitive control: Meta-analyses of switching and Stroop studies. Human Brain Mapping, 25(1), 22-34.

Dikeos, D. G., Wickham, H., McDonald, C., Walshe, M., Sigmundsson, T., Bramon, E., et al. (2006). Distribution of symptom dimensions across Kraepelinian divisions. The British Journal of Psychiatry, $189,346-353$.

Docherty, N. M., \& Gordinier, S. W. (1999). Immediate memory, attention and communication disturbances in schizophrenia patients and their relatives. Psychological Medicine, 29(1), 189-197.

*Donohoe, G., Corvin, A., \& Robertson, I. H. (2006). Evidence that specific executive functions predict symptom variance among schizophrenia patients with a predominantly negative symptom profile. Cognitive Neuropsychiatry 11(1), 13-32.

Duncan, G. E., Sheitman, B. B., \& Lieberman, J. A. (1999). An integrated view of pathophysiological models of schizophrenia. Brain Research. Brain Research Reviews, 29(2-3), 250-264.

Ebmeier, K. P., Blackwood, D. H., Murray, C., Souza, V., Walker, M., Dougall, N., et al. (1993). Singlephoton emission computed tomography with $99 \mathrm{mTc}$-exametazime in unmedicated schizophrenic patients. Biological Psychiatry, 33(7), 487-495.

*Eckman, P. S., \& Shean, G. D. (2000). Impairment in test performance and symptom dimensions of schizophrenia. Journal of Psychiatric Research, 34(2), 147-153.

*Ehmann, T. S., Khanbhai, I., Macewan, G. W., Smith, G. N., Honer, W. G., Flynn, S., et al. (2004). Neuropsychological correlates of the PANSS Cognitive Factor. Psychopathology, 37(5), 253-258.

Endicott, J., \& Spitzer, R. L. (1978). A diagnostic interview: The schedule for affective disorders and schizophrenia. Archives of General Psychiatry, 35(7), 837-844.

Fioravanti, M., Carlone, O., Vitale, B., Cinti, M. E., \& Clare, L. (2005). A meta-analysis of cognitive deficits in adults with a diagnosis of schizophrenia. Neuropsychology Review, 15(2), 73-95.

*Franke, P., Maier, W., Hain, C., \& Klingler, T. (1992). Wisconsin card sorting test: An indicator of vulnerability to schizophrenia? Schizophrenia Research, 6(3), 243-249.

Freeman, D., Garety, P. A., \& Kuipers, E. (2001). Persecutory delusions: developing the understanding of belief maintenance and emotional distress. Psychological Medicine, 31(7), 1293-1306.

Frith, C. D. (2004). Schizophrenia and theory of mind. Psychological Medicine, 34(3), 385-389.

Garety, P. A., \& Freeman, D. (1999). Cognitive approaches to delusions: A critical review of theories and evidence. The British Journal of Clinical Psychology, 38(Pt 2), 113-154.

Garety, P. A., Kuipers, E., Fowler, D., Freeman, D., \& Bebbington, P. E. (2001). A cognitive model of the positive symptoms of psychosis. Psychological Medicine, 31(2), 189-195.

Gold, J. M., Carpenter, C., Randolph, C., Goldberg, T. E., \& Weinberger, D. R. (1997). Auditory working memory and Wisconsin Card Sorting Test performance in schizophrenia. Archives of General Psychiatry, 54, 159-165.

Good, K. P., Rabinowitz, J., Whitehorn, D., Harvey, P. D., DeSmedt, G., \& Kopala, L. C. (2004). The relationship of neuropsychological test performance with the PANSS in antipsychotic naive, firstepisode psychosis patients. Schizophrenia Research, 68(1), 11-19.

Green, M. F. (1996). What are the functional consequences of neurocognitive deficits in schizophrenia? American Journal of Psychiatry, 153(3), 321-330.

Grube, B. S., Bilder, R. M., \& Goldman, R. S. (1998). Meta-analysis of symptom factors in schizophrenia. Schizophrenia Research, 31(2-3), 113-120.

*Guillem, F., Bicu, M., Bloom, D., Wolf, M. A., Desautels, R., Lalinec, M., et al. (2001). Neuropsychological impairments in the syndromes of schizophrenia: A comparison between different dimensional models. Brain and Cognition, 46(1-2), 153-159.

Hamilton, M. (1960). A rating scale for depression. Journal of Neurology, Neurosurgery and Psychiatry, 30 , 56-62.

*Hammer, M. A., Katsanis, J., \& Iacono, W. G. (1995). The relationship between negative symptoms and neuropsychological performance. Biological Psychiatry, 37(11), 828-830.

Heaton, R. K. (1981). A manual for the Wisconsin card sorting test. Odessa, FL: Psychological Assessment Resources.

Heaton, R. K. (1985). Wisconsin card sorting test. Odessa, FL: Psychological Assessment Resources.

Hedges, L. V., \& Vevea, J. L. (1998). Fixed- and random-effects models in meta-analysis. Psychological Methods, 3, 486-504. 
Heinrichs, R. W., \& Zakzanis, K. K. (1998). Neurocognitive deficit in schizophrenia: A quantitative review of the evidence. Neuropsychology, 12(3), 426-445.

*Heydebrand, G., Weiser, M., Rabinowitz, J., Hoff, A. L., DeLisi, L. E., \& Csernansky, J. G. (2004). Correlates of cognitive deficits in first episode schizophrenia. Schizophrenia Research, 68(1), 1-9.

*Himelhoch, S., Taylor, S. F., Goldman, R. S., \& Tandon, R. (1996). Frontal lobe tasks, antipsychotic medication, and schizophrenia syndromes. Biological Psychiatry, 39(3), 227-229.

*Holthausen, E. A., Wiersma, D., Knegtering, R. H., \& van den Bosch, R. J. (1999). Psychopathology and cognition in schizophrenia spectrum disorders: The role of depressive symptoms. Schizophrenia Research, 39(1), 65-71.

*Ihara, H., Berrios, G. E., \& McKenna, P. J. (2003). The association between negative and dysexecutive syndromes in schizophrenia: A cross-cultural study. Behavioural Neurology, 14(3-4), 63-74.

*Ito, M., Kanno, M., Mori, Y., \& Niwa, S. (1997). Attention deficits assessed by continuous performance test and span of apprehension test in japanese schizophrenic patients. Schizophrenia Research, 23(3), 205211.

Johns, L. C., Rossell, S., Frith, C., Ahmad, F., Hemsley, D., Kuipers, E., et al. (2001). Verbal self-monitoring and auditory verbal hallucinations in patients with schizophrenia. Psychological Medicine, 31(4), 705715 .

Johnson Selfridge, M., \& Zalewski, C. (2001). Moderator variables of executive functioning in schizophrenia: Meta-analytic findings. Schizophrenia Bulletin, 27(2), 305-316.

Josin, G. M., \& Liddle, P. F. (2001). Neural network analysis of the pattern of functional connectivity between cerebral areas in schizophrenia. Biological Cybernetics, 84(2), 117-122.

Kaufman, A. S., \& Kaufman, N. L. (1990). Manual for the Kaufman Brief Intelligent Test (K-BIT). Circle Pines, MN: American Guidance Service.

Kay, S. R., Fiszbein, A., \& Opler, L. A. (1987). The positive and negative syndrome scale (PANSS) for schizophrenia. Schizophrenia Bulletin, 13(2), 261-276.

*Keefe, R. S., Bilder, R. M., Harvey, P. D., Davis, S. M., Palmer, B. W., Gold, J. M., et al. (2006). Baseline neurocognitive deficits in the CATIE schizophrenia trial. Neuropsychopharmacology, 31(9), 2033-2046.

Kellendonk, C., Simpson, E. H., Polan, H. J., Malleret, G., Vronskaya, S., Winiger, V., et al. (2006). Transient and selective overexpression of dopamine $\mathrm{D} 2$ receptors in the striatum causes persistent abnormalities in prefrontal cortex functioning. Neuron, 49(4), 603-615.

Kerns, J. G., \& Berenbaum, H. (2002). Cognitive impairments associated with formal thought disorder in people with schizophrenia. Journal of Abnormal Psychology, 111(2), 211-224.

Krabbendam, L., Arts, B., van Os, J., \& Aleman, A. (2005). Cognitive functioning in patients with schizophrenia and bipolar disorder: A quantitative review. Schizophrenia Research, 80(2-3), 137-149.

Krawiecka, M., Goldberg, D., \& Vaughan, M. (1977). A standardized psychiatric assessment scale for rating chronic psychotic patients. Acta Psychiatrica Scandinavica, 55(4), 299-308.

*Krishnadas, R., Moore, B. P., Nayak, A., \& Patel, R. R. (2007). Relationship of cognitive function in patients with schizophrenia in remission to disability: A cross-sectional study in an Indian sample. Annals of General Psychiatry, 6, 19.

Kuipers, E., Garety, P., Fowler, D., Freeman, D., Dunn, G., \& Bebbington, P. (2006). Cognitive, emotional, and social processes in psychosis: Refining cognitive behavioral therapy for persistent positive symptoms. Schizophrenia Bulletin, 32 Suppl 1, S24-31.

Laruelle, M., Kegeles, L. S., \& Abi-Dargham, A. (2003). Glutamate, dopamine, and schizophrenia: From pathophysiology to treatment. Annals of the New York Academy of Sciences, 1003, 138-158.

Lawrie, S. M., \& Abukmeil, S. S. (1998). Brain abnormality in schizophrenia. A systematic and quantitative review of volumetric magnetic resonance imaging studies. The British Journal of Psychiatry, 172, 110120.

Lee, J., \& Park, S. (2005). Working memory impairments in schizophrenia: A meta-analysis. Journal of Abnormal Psychology, 114(4), 599-611.

Lennox, B. R., Park, S. B., Medley, I., Morris, P. G., \& Jones, P. B. (2000). The functional anatomy of auditory hallucinations in schizophrenia. Psychiatry Research, 100(1), 13-20.

*Less-Roitman, S. E., Keefe, R. S., Harvey, P. D., Siever, L. J., \& Mohs, R. C. (1997). Attentional and eye tracking deficits correlate with negative symptoms in schizophrenia. Schizophrenia Research, 26(2-3), $139-146$ 
Liddle, P. F. (1987). The symptoms of chronic schizophrenia. A re-examination of the positive-negative dichotomy. The British Journal of Psychiatry, 151, 145-151.

Liddle, P. F. (1992). Signs and symptoms of psychotic illness. Hammersmith Hospital, London. (Questionnaire available from the author on request.).

Liddle, P. F., Friston, K. J., Frith, C. D., \& Frackowiak, R. S. (1992). Cerebral blood flow and mental processes in schizophrenia. Journal of the Royal Society of Medicine, 85(4), 224-227.

Liddle, P. F., Friston, K. J., Frith, C. D., Hirsch, S. R., Jones, T., \& Frackowiak, R. S. (1992). Patterns of cerebral blood flow in schizophrenia. The British Journal of Psychiatry, 160, 179-186.

*Liddle, P. F., \& Morris, D. L. (1991). Schizophrenic syndromes and frontal lobe performance. The British Journal of Psychiatry, 158, 340-345.

Lieberman, J., Chakos, M., Wu, H., Alvir, J., Hoffman, E., Robinson, D., et al. (2001). Longitudinal study of brain morphology in first episode schizophrenia. Biological Psychiatry, 49(6), 487-499.

Lindenmayer, J. P., Grochowski, S., \& Hyman, R. B. (1995). Five factor model of schizophrenia: Replication across samples. Schizophrenia Research, 14(3), 229-234.

*Lucas, S., Fitzgerald, D., Redoblado Hodge, M. A., Anderson, J., Sanbrook, M., Harris, A., et al. (2004). Neuropsychological correlates of symptom profiles in first episode schizophrenia. Schizophrenia Research, 71(2-3), 323-330.

*Mahurin, R. K., Velligan, D. I., Hazleton, B., Davis, J. M., Eckert, S., \& Miller, A. L. (2006). Trail making test errors and executive function in schizophrenia and depression. The Clinical neuropsychologist 20(2), 271-288.

McGorry, P. D., Bell, R. C., Dudgeon, P. L., \& Jackson, H. J. (1998). The dimensional structure of first episode psychosis: an exploratory factor analysis. Psychological Medicine, 28(4), 935-947.

McGuire, P. K., Silbersweig, D. A., Wright, I., Murray, R. M., David, A. S., Frackowiak, R. S., et al. (1995). Abnormal monitoring of inner speech: A physiological basis for auditory hallucinations. Lancet, 346(8975), 596-600.

Menon, V., Anagnoson, R. T., Glover, G. H., \& Pfefferbaum, A. (2001). Functional magnetic resonance imaging evidence for disrupted basal ganglia function in schizophrenia. American Journal of Psychiatry, 158(4), 646-649.

*Minzenberg, M. J., Pool, J. H., Vinogradov, S., Shenaut, G. K., \& Ober, B. A. (2003). Slowed lexical access is uniquely associated with positive and disorganised symptoms in schizophrenia. Cognitive Neuropsychiatry, 8(2), 107-127.

*Moritz, S., Andresen, B., Jacobsen, D., Mersmann, K., Wilke, U., Lambert, M., et al. (2001). Neuropsychological correlates of schizophrenic syndromes in patients treated with atypical neuroleptics. European Psychiatry 16(6), 354-361.

*Moritz, S., Heeren, D., Andresen, B., \& Krausz, M. (2001). An analysis of the specificity and the syndromal correlates of verbal memory impairments in schizophrenia. Psychiatry Research, 101(1), 23-31.

Mortimer, A. M., McKenna, P. J., Lund, C. E., \& Mannuzza, S. (1989). Rating of negative symptoms using the High Royds evaluation of negativity (HEN) scale. The British Journal of Psychiatry. Supplement(7), $89-92$.

Murray, V., McKee, I., Miller, P. M., Young, D., Muir, W. J., Pelosi, A. J., et al. (2005). Dimensions and classes of psychosis in a population cohort: a four-class, four-dimension model of schizophrenia and affective psychoses. Psychological Medicine, 35(4), 499-510.

Myin-Germeys, I., \& van Os, J. (2007). Stress-reactivity in psychosis: Evidence for an affective pathway to psychosis. Clinical Psychology Review, 27(4), 409-424.

Myin Germeys, I., Krabbendam, L., Jolles, J., Delespaul, P. A., \& van Os, J. (2002). Are cognitive impairments associated with sensitivity to stress in schizophrenia? An experience sampling study. American Journal of Psychiatry, 159(3), 443-449.

Nelson, H. E. (1976). A modified card sorting test sensitive to frontal lobe defects. Cortex, 12(313-324).

Nelson, H. E. \& O'Connell, A. (1978). Dementia: The estimation of premorbid intelligence levels using the National Adult Reading Test. Cortex, 14, 234-244.

*Nelson, H. E., Pantelis, C., Carruthers, K., Speller, J., Baxendale, S., \& Barnes, T. R. (1990). Cognitive functioning and symptomatology in chronic schizophrenia. Psychological Medicine, 20(2), 357-365.

Nieuwenstein, M. R., Aleman, A., \& de Haan, E. H. (2001). Relationship between symptom dimensions and neurocognitive functioning in schizophrenia: A meta-analysis of WCST and CPT studies. Wisconsin Card Sorting Test. Continuous Performance Test. Journal of Psychiatric Research, 35(2), 119-125. 
*Norman, R. M., Malla, A. K., Morrison Stewart, S. L., Helmes, E., Williamson, P. C., Thomas, J., et al. (1997). Neuropsychological correlates of syndromes in schizophrenia. The British Journal of Psychiatry, 170, 134-139.

Nuechterlein, K. H., Barch, D. M., Gold, J. M., Goldberg, T. E., Green, M. F., \& Heaton, R. K. (2004). Identification of separable cognitive factors in schizophrenia. Schizophrenia Research, 72(1), 29-39.

Nuechterlein, K. H., \& Dawson, M. E. (1984). A heuristic vulnerability/stress model of schizophrenic episodes. Schizophrenia Bulletin, 10(2), 300-312.

*Nuechterlein, K. H., Edell, W. S., Norris, M., \& Dawson, M. E. (1986). Attentional vulnerability indicators, thought disorder, and negative symptoms. Schizophrenia Bulletin, 12(3), 408-426.

*O'Leary, D. S., Flaum, M., Kesler, M. L., Flashman, L. A., Arndt, S., \& Andreasen, N. C. (2000). Cognitive correlates of the negative, disorganized, and psychotic symptom dimensions of schizophrenia. The Journal of Neuropsychiatry and Clinical Neurosciences, 12(1), 4-15.

Overall, J. E., \& Gorham, D. R. (1962). The brief psychiatric rating scale. Psychological Reports, 10, 799812.

*Pandurangi, A. K., Sax, K. W., Pelonero, A. L., \& Goldberg, S. C. (1994). Sustained attention and positive formal thought disorder in schizophrenia. Schizophrenia Research, 13(2), 109-116.

Pelletier, M., Achim, A. M., Montoya, A., Lal, S., \& Lepage, M. (2005). Cognitive and clinical moderators of recognition memory in schizophrenia: A meta-analysis. Schizophrenia Research, 74(2-3), 233-252.

Peralta, V., Cuesta, M. J., \& de Leon, J. (1994). An empirical analysis of latent structures underlying schizophrenic symptoms: A four-syndrome model. Biological Psychiatry, 36(11), 726-736.

*Perry, W., \& Braff, D. L. (1998). A multimethod approach to assessing perseverations in schizophrenia patients. Schizophrenia Research, 33(1-2), 69-77.

*Ragland, J. D., Censits, D. M., Gur, R. C., Glahn, D. C., Gallacher, F., \& Gur, R. E. (1996). Assessing declarative memory in schizophrenia using Wisconsin card sorting test stimuli: The paired associate recognition test. Psychiatry Research, 60(2-3), 135-145.

Raudenbush, S. W. (1994). Random effects models. In H. M. Cooper \& L. V. Hedges (Eds.), The handbook of research synthesis (pp. 301-321). New York: Russell Sage.

Reitan, R. M. (1958). Validity of the trail making test as an indication of organic brain damage. Perceptual and Motor Skills, 8, 271-276.

Rey, A. (1941). L'examen psychologique dans les cas d'encephalopathie traumatique: les problèmes (The psychological examination in cases of traumatic encephalopathy: problems). Archives de Psychologie, 28, 215- 285.

Rey, A. (1964). L'examen Clinique en Psychologie (Clinical Assessment in Psychology). Paris: Presses Universitaires de France.

*Rocca, P., Bellino, S., Calvarese, P., Marchiaro, L., Patria, L., Rasetti, R., et al. (2005). Depressive and negative symptoms in schizophrenia: Different effects on clinical features. Comprehensive Psychiatry, 46(4), 304-310.

*Rocca, P., Castagna, F., Marchiaro, L., Rasetti, R., Rivoira, E., \& Bogetto, F. (2006). Neuropsychological correlates of reality distortion in schizophrenic patients. Psychiatry Research, 145(1), 49-60.

*Rosse, R. B., Schwartz, B. L., Kim, S. Y., \& Deutsch, S. I. (1993). Correlation between antisaccade and Wisconsin card sorting test performance in schizophrenia. American Journal of Psychiatry, 150(2), 333335.

*Rund, B. R., Melle, I., Friis, S., Larsen, T. K., Midboe, L. J., Opjordsmoen, S., et al. (2004). Neurocognitive dysfunction in first-episode psychosis: Correlates with symptoms, premorbid adjustment, and duration of untreated psychosis. American Journal of Psychiatry, 161(3), 466-472.

Sanfilipo, M., Lafargue, T., Rusinek, H., Arena, L., Loneragan, C., Lautin, A., et al. (2000). Volumetric measure of the frontal and temporal lobe regions in schizophrenia: Relationship to negative symptoms. Archives of General Psychiatry, 57(5), 471-480.

*Sanfilipo, M., Lafargue, T., Rusinek, H., Arena, L., Loneragan, C., Lautin, A., et al. (2002). Cognitive performance in schizophrenia: Relationship to regional brain volumes and psychiatric symptoms. Psychiatry Research, 116(1-2), 1-23.

Schroder, J., Buchsbaum, M. S., Siegel, B. V., Geider, F. J., Lohr, J., Tang, C., et al. (1996). Cerebral metabolic activity correlates of subsyndromes in chronic schizophrenia. Schizophrenia Research, 19(1), 41-53. 
Schultz, S. K., Miller, D. D., Oliver, S. E., Arndt, S., Flaum, M., \& Andreasen, N. C. (1997). The life course of schizophrenia: Age and symptom dimensions. Schizophrenia Research, 23(1), 15-23.

Serretti, A., De Ronchi, D., Lorenzi, C., \& Berardi, D. (2004). New antipsychotics and schizophrenia: A review on efficacy and side effects. Current Medicinal Chemistry, 11(3), 343-358.

Serretti, A., Rietschel, M., Lattuada, E., Krauss, H., Schulze, T. G., Muller, D. J., et al. (2001). Major psychoses symptomatology: Factor analysis of 2241 psychotic subjects. European Archives of Psychiatry and Clinical Neuroscience, 251(4), 193-198.

Shergill, S. S., Brammer, M. J., Williams, S. C., Murray, R. M., \& McGuire, P. K. (2000). Mapping auditory hallucinations in schizophrenia using functional magnetic resonance imaging. Archives of General Psychiatry, 57(11), 1033-1038.

Shergill, S. S., Bullmore, E., Simmons, A., Murray, R., \& McGuire, P. (2000). Functional anatomy of auditory verbal imagery in schizophrenic patients with auditory hallucinations. American Journal of Psychiatry, 157(10), 1691-1693.

*Simon Md, A. E., Giacomini, V., Ferrero, F., \& Mohr, S. (2003). Is executive function associated with symptom severity in schizophrenia? European Archives of Psychiatry and Clinical Neuroscience, 253(4), 216-218.

Simonsen, E., Friis, S., Haahr, U., Johannessen, J. O., Larsen, T. K., Melle, I., et al. (2007). Clinical epidemiologic first-episode psychosis: 1-year outcome and predictors. Acta Psychiatrica Scandinavica, 116(1), 54-61.

Sitskoorn, M. M., Aleman, A., Ebisch, S. J., Appels, M. C., \& Kahn, R. S. (2004). Cognitive deficits in relatives of patients with schizophrenia: A meta-analysis. Schizophrenia Research, 71(2-3), 285-295.

Slosson, R. L. (1963). Slosson Intelligence Test for Children and Adults. East Aurora, NY: Slosson Education Publications.

Spitzer, R. L., Endicott, J., \& Robins, E. (1978). Research diagnostic criteria: Rationale and reliability. Archives of General Psychiatry, 35(6), 773-782.

Steiger, J. H. (1980). Test for comparing elements of a correlation matrix. Psychological Bulletin, 87, 245251.

Sterne, J., \& Egger, M. (2005). Regression methods to detect publication and other bias in meta-analysis. In A. J. S. H.R. Rothstein and M. Borenstein (Eds.), Publication bias in meta-analysis: Prevention, assessment and adjustments (pp. 99-110). New York: Wiley.

*Strauss, M. E., Buchanan, R. W., \& Hale, J. (1993). Relations between attentional deficits and clinical symptoms in schizophrenic outpatients. Psychiatry Research, 47(3), 205-213.

Stroop, J. R. (1935). Studies of interference in serial verbal reactions. Journal of Experimental Psychology, $18,643-662$.

Szoke, A., Schurhoff, F., Mathieu, F., Meary, A., Ionescu, S., \& Leboyer, M. (2005). Tests of executive functions in first-degree relatives of schizophrenic patients: A meta-analysis. Psychological Medicine, 35(6), 771-782.

*Tandon, R., DeQuardo, J. R., Taylor, S. F., McGrath, M., Jibson, M., Eiser, A., et al. (2000). Phasic and enduring negative symptoms in schizophrenia: Biological markers and relationship to outcome. Schizophrenia Research, 45(3), 191-201.

Test, M. A., Knoeder, W. H., Allness, D. J., Burke, S. S., Brown, R. L., \& Wallisch, L. S. (1991). Long-term community care through an assertive continuous treatment team. In C. Taminga \& S. C. Schulz (Eds.), Advance in neuropsychiatry and psychopharmacology (Vol. I, pp. 239-246). New York: Raven Press.

*Torres, I. J., O'Leary, D. S., \& Andreasen, N. C. (2004). Symptoms and interference from memory in schizophrenia: Evaluation of Frith's model of willed action. Schizophrenia Research, 69(1), 35-43.

van Dael, F., Versmissen, D., Janssen, I., Myin-Germeys, I., van Os, J., \& Krabbendam, L. (2006). Data gathering: Biased in psychosis? Schizophrenia Bulletin, 32(2), 341-351.

van Hooren, S., Versmissen, D., Janssen, I., Myin-Germeys, I., Campo, J. A., Mengelers, R., et al. Social cognition and neurocognition as independent domains in psychosis. Schizophrenia Research, 103, 257265.

Versmissen, D., Janssen, I., Myin-Germeys, I., Mengelers, R., Campo, J. A., van Os, J., et al. (2007). Evidence for a relationship between mentalising deficits and paranoia over the psychosis continuum. Schizophrenia Research, 99(1-3), 103-110.

*Voruganti, L. N., Heslegrave, R. J., \& Awad, A. G. (1997). Neurocognitive correlates of positive and negative syndromes in schizophrenia. Canadian Journal of Psychiatry 42(10), 1066-1071. 
Wechsler, D. (1955). Wechsler adult intelligence scale. New York: Manual Psychological Corporation.

Wechsler, D. (1981). Wechsler adult intelligence scale-Revised. New York: Psychological Corporation.

Wechsler, D. (1987). Wechsler memory scale-Revised manual. New York: The Psychological Corporation.

Wechsler, D. (1997). WAIS-III: Wechsler adult intelligence scale, administration and scoring manual, 3rd ed. New York: Psychological Corporation.

Weinberger, D. R., \& Berman, K. F. (1988). Speculation on the meaning of cerebral metabolic hypofrontality in schizophrenia. Schizophrenia Bulletin, 14(2), 157-168.

Weinberger, D. R., Berman, K. F., \& Illowsky, B. P. (1988). Physiological dysfunction of dorsolateral prefrontal cortex in schizophrenia. III. A new cohort and evidence for a monoaminergic mechanism. Archives of General Psychiatry, 45(7), 609-615.

Weinberger, D. R., Berman, K. F., \& Zec, R. F. (1986). Physiologic dysfunction of dorsolateral prefrontal cortex in schizophrenia. I. Regional cerebral blood flow evidence. Archives of General Psychiatry, 43(2), 114-124.

Wing, J., Cooper, J., \& Sartorius, N. (Eds.). (1974). The measurement and classification of psychiatric symptoms. Cambridge: Cambridge University Press.

*Woodward, T. S., Ruff, C. C., Thornton, A. E., Moritz, S., \& Liddle, P. F. (2003). Methodological considerations regarding the association of Stroop and verbal fluency performance with the symptoms of schizophrenia. Schizophrenia Research, 61(2-3), 207-214.

*Woodward, T. S., Thornton, A. E., Ruff, C. C., Moritz, S., \& Liddle, P. F. (2004). Material-specific episodic memory associates of the psychomotor poverty syndrome in schizophrenia. Cognitive Neuropsychiatry, 9(3), 213-227.

World Health Organization. (1977). International classification of diseases (9th revision). Ginebra: World Health Organization.

World Health Organization. (1990). International classification of diseases (10th revision). Ginebra: World Health Organization. 



\title{
CHAPTER 3
}

\author{
EVIDENCE THAT ONSET OF CLINICAL \\ PSYCHOSIS IS AN OUTCOME OF \\ PROGRESSIVELY MORE PERSISTENT \\ SUBCLINICAL PSYCHOTIC \\ EXPERIENCES: AN 8-YEAR COHORT \\ STUDY
}

Maria de Gracia Dominguez ${ }^{1}$, Marieke Wichers ${ }^{1}$, Roselind Lieb ${ }^{2,5}$, Hans-Ulrich Wittchen ${ }^{2,3}$, Jim van Os ${ }^{1,4}$

${ }^{1}$ Department of Psychiatry and Neuropsychology, South Limburg Mental Health Research and Teaching Network, EURON, Maastricht University Medical Centre, Maastricht, The Netherlands.

${ }^{2}$ Max Planck Institute of Psychiatry, Clinical Psychology and Epidemiology Unit, Munich, Germany.

${ }^{3}$ Institute of Clinical Psychology and Psychotherapy, Technical University Dresden, Dresden, Germany. ${ }^{4}$ Division of Psychological Medicine, Institute of Psychiatry, London, UK.

${ }^{5}$ Epidemiology and Health Psychology, Institute of Psychology, University of Basel, Basel, Switzerland.

Schizophrenia Bulletin doi:10.1093/Schbul/sbp022 


\begin{abstract}
Objective

This study examined the hypothesis that developmental expression of psychometric risk in the form of subclinical psychotic experiences in the general population is usually transitory, but in some instances may become abnormally persistent and progress to a clinical psychotic state.
\end{abstract}

\title{
Method
}

A prospective cohort study was conducted in a general population sample of 845 adolescents, aged 14-17 years, in Munich, Germany (Early Developmental Stages of Psychopathology Study). Expression of psychosis was assessed four times (T0T3) over a period of 8.4 years. Transition from subclinical psychosis at T0-T2 to clinical psychosis in terms of impairment at T3 was examined as a function of the level of prior persistence of subclinical psychosis (present never, once, twice or thrice).

\section{Results}

The more subclinical psychosis persisted over the period T0-T2, the greater the risk of transition to clinical psychosis at $\mathrm{T} 3$ in a dose-response fashion (subclinical psychosis expression once over T0-T2: OR=1.5 (95\%CI: 0.6-3.7), post-test probability $(\mathrm{PP})=5 \%$; twice: $\mathrm{OR}=5.0(95 \% \mathrm{CI}: 1.6-15.9), \mathrm{PP}=16 \%$; at all three measurements: $\mathrm{OR}=9.9(95 \% \mathrm{CI}: 2.5-39.8), \mathrm{PP}=27 \%)$. Of all clinical psychosis at T3, more than a third $(38.3 \%)$ was preceded by subclinical psychotic experiences at least once and a fifth (19.6\%) at least twice.

\section{Conclusions}

Consequently, a significant proportion of psychotic disorder may be conceptualized as the rare poor outcome of a common developmental phenotype characterised by persistence of psychometrically detectable subclinical psychotic experiences. This may be summarized descriptively as a psychosis proneness-persistence-impairment model of psychotic disorder.

\section{Key words}

Epidemiology, General Population, Psychosis Onset, Adolescence, Transition to Clinical Relevance, Subclinical Psychosis. 


\section{Introduction}

Psychosis is commonly considered a rare phenomenon outside the range of normal human experience, giving rise to significant disability. However, the type of distribution expected for disorders of multifactorial interactive aetiologies is continuous (Johns \& van Os, 2001; van Os, Linscott, Myin-Germeys, Delespaul, \& Krabbendam, 2009) and many mental disorders have accordingly been shown to exist along a spectrum including subclinical expressions, evidenced by, eg, relatively high rates of expression of bipolar spectrum symptoms, subthreshold depressive states and social restriction associated with autism (Akiskal \& Vazquez, 2006; Angst \& Cassano, 2005; Constantino \& Todd, 2003; Judd \& Akiskal, 2003).

Similar findings have been reported for psychosis (Johns \& van Os, 2001). The clinical psychosis phenotype has not only been shown to be much less rare than previously thought (Perala et al., 2007) but is also thought to be expressed at levels well below its clinical manifestation (Chapman, Chapman, Kwapil, Eckblad, \& Zinser, 1994; Stefanis et al., 2002; van Os, Hanssen, Bijl, \& Ravelli, 2000; Yung et al., 2003). Apart from strong evidence of phenomenological continuity in form and structure (Johns \& van Os, 2001), continuity between clinical and subclinical phenotypes is further suggested by: (i) similar associations with demographic factors, in particular the negative association with age and the positive association with single marital status and social disadvantage (Johns \& van Os, 2001; Peters, Joseph, \& Garety, 1999; van Os et al., 2000; Verdoux et al., 1998), (ii) transitions over time from the subclinical to the clinical (Chapman et al., 1994; M. Hanssen, Bak, Bijl, Vollebergh, \& van Os, 2005; Poulton et al., 2000), (iii) familial coclustering of the clinical and the subclinical (Kendler \& Diehl, 1993), (iv) strong dose-response effects of the urban environment on both phenotypes (Spauwen, Krabbendam, Lieb, Wittchen, \& van Os, 2006), (v) sharing of cognitive and motor deficits (Krabbendam, Myin-Germeys, Hanssen, \& van Os, 2005; Voglmaier et al., 2000), (vi) sharing of risk genes (Stefanis et al., 2004) and of environmental risk factors such as cannabis (Fergusson, Horwood, \& Swain-Campbell, 2003).

Many questions remain, however, with regard to the hypothesized continuity over time from subclinical to clinical psychotic states. The seminal study by Poulton and colleagues demonstrated that more than $25 \%$ of participants with low grade psychotic experiences at age 11 years developed a clinical psychotic disorder by age 26 years (Poulton et al., 2000). However, an equally important conclusion of the study was that the great majority of children with expression of psychotic experiences would never develop a psychotic disorder-psychosis was only a transitory developmental state for most. Similar conclusions were reached by other, less lengthy follow-up studies in adult population cohorts, with transition rates of around 10\% (Chapman et al., 1994; M. Hanssen et al., 2005). Cougnard et al (2007) 
recently suggested that one of the reasons why most developmental expression of psychosis remains transitory is that exposure to additional environmental risks over development, such as cannabis use, childhood trauma and urban environment (Cougnard et al., 2007), is necessary in order for subclinical psychosis to first become persistent and, second, to deteriorate into a clinical psychotic state (Figure 1). However, to date, no evidence has been presented suggesting that abnormal persistence of a common and normally transitory developmental subclinical psychosis phenotype is the precursor state of clinical deterioration.

In the current paper, it was examined whether subclinical psychotic experiences with a greater tendency to persist over time would have a greater probability to become clinically relevant in terms of dysfunction and need for care (impairment). The hypothesis of greater persistence resulting in greater probability of transition from subclinical to clinical psychosis characterised by impairment was tested using a prospective, observational study in a large, general population sample of adolescents followed over a period of more than 8 years.

Figure 1. Study Design

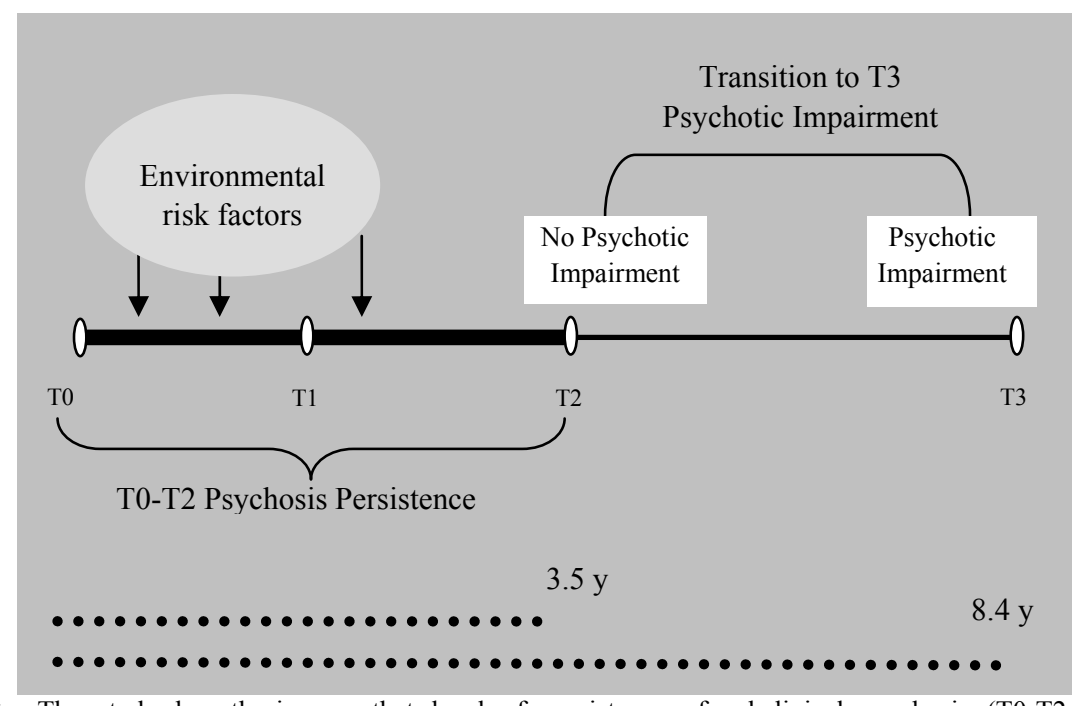

Note. The study hypothesis was that level of persistence of subclinical psychosis (T0-T2 psychosis persistence) over a period of $3.5 \mathrm{y}$, that previous work showed is associated with exposure to environmental risk factors (Cougnard et al., 2007), impacts on the risk of transition to T3 psychotic impairment approximately 5 y later. Psychosis persistence (defined as presence of the $10 \%$ highest score of psychotic experiences of the symptom check list-psychosis subscale): never, sporadic (once), recurrent (twice), persistent (thrice) over the period T0-T2. Psychotic impairment: a variable scored "0" in subjects with psychotic experiences who scored " 0 " on both help seeking and dysfunction associated with the symptom and " 1 " in subjects scoring " 1 " on either or both of help seeking and dysfunction. 


\section{Method}

\section{Sample}

Data came from the Early Developmental Stages of Psychopathology (EDSP) Study, which collected data on the prevalence, incidence, risk factors, comorbidity and course of mental disorders in a random, representative population sample of adolescents and young adults in the general population. The baseline sample, following ethics committee approval, was randomly drawn, in 1994, from the respective population registry offices of Munich and its 29 counties, to mirror the distribution of individuals expected to be 14-24 years of age at the time of the baseline (T0) interview in 1995 . The base population were all those born between June 1, 1970 and May 31, 1981, registered as residents in these localities and having German citizenship. These registers can be regarded as highly accurate because (1) each German is registered by his town, (2) they are regularly updated, (3) in the interest of scientific studies, any number of randomly drawn addresses with a given sex and age-group can be obtained, and (4) strict enforcement of registration by law and the police applies. More details on the sampling, representativeness, instruments, procedures and statistical methods of the EDSP Study sample have previously been presented (Lieb, Isensee, von Sydow, \& Wittchen, 2000; Wittchen, Perkonigg, Lachner, \& Nelson, 1998).

\section{Study Design}

The overall design of this cohort study is longitudinal and prospective, consisting of a baseline (T0) and 3 follow-up surveys, covering a time period of on average 1.6 years $(\mathrm{T} 0-\mathrm{T} 1, \mathrm{SD}=0.2), 3.5$ years $(\mathrm{T} 0-\mathrm{T} 2, \mathrm{SD}=0.3)$ and 8.4 years $(\mathrm{T} 0-\mathrm{T} 3$, range 7.3 10.5 years, $\mathrm{SD}=0.7)$.

Because the primary goal of the study was to examine the incidence and developmental risk factors for psychopathology, the younger group (14-15 y), presumed to have the highest incidence density, were sampled at twice the rate of persons aged 16-21 years, and the oldest group (22-24 y) was sampled at half this rate. For the same reason, subjects aged 14-17 years at baseline were examined at the 4 time points, and subjects aged 18-24 years were assessed only 3 times.

The present study is based on a subset of EDSP Study respondents, namely, the younger cohort (aged 14-17 y at baseline) only (T0, $n=1395$, response rate:75\%), thus ensuring an adolescent population at risk of developing psychotic experiences and subsequent transition to clinically relevant psychotic states over 4 assessment periods. Response rates for the younger cohort were respectively $88 \%$ at $\mathrm{T} 1$ $(\mathrm{n}=1228), 83 \%$ at T2 $(\mathrm{n}=1169)$ and $73 \%$ at T3 $(\mathrm{n}=1022)$. After description of the study to the subjects, written informed consent was obtained. 


\section{Instruments}

\section{The Self-report Symptom Checklist-90-R}

At all time points, participants completed the self-report Symptom Checklist-90-R (SCL-90-R), a comprehensive self-report symptom inventory, multidimensional in nature, and oriented to screen for a broad range of psychological problems and psychopathology in community respondents and respondents with somatic and psychiatric disorders. It contains 90 items, scored on a 5-point severity scale, measuring 9 primary symptom dimensions named 'somatization', 'obsessivecompulsive', 'interpersonal sensitivity', 'depression', 'anxiety', 'hostility', 'phobic anxiety', 'paranoid ideation' and 'psychoticism'. Reliability and validity of the SCL-90-R were established previously (Derogatis \& Cleary, 1977). The time frame is the past 2 weeks.

\section{The Munich-Composite International Diagnostic Interview}

Participants were also assessed using the computerized version of the MunichComposite International Diagnostic Interview (DIA-X/M-CIDI) (Wittchen \& Pfister, 1997), an updated version of the World Health Organization's CIDI version 1.2 (World Health Organization, 1990). The DIA-X/M-CIDI is a comprehensive, fully standardized diagnostic interview and assesses symptoms, syndromes, and diagnoses of various mental disorders in accordance with the definitions and criteria of the International Classification of Diseases, Tenth Revision, and Diagnostic and Statistical Manual of Mental Disorders (Fourth edition), along with information about onset, duration, severity of symptoms, and psychosocial impairment. The CIDI has been primarily designed for use in epidemiological studies of mental disorders and can also be used for clinical purposes. It is divided into 16 sections: 1 sociodemographic section, 12 sections assessing 288 symptoms of groups of mental disorders (including 'somatoform and dissociative', 'phobic and other anxiety', 'depressive and dysthymic', 'manic and bipolar affective', 'cchizophrenia and other psychotic', 'eating', 'dementia and other cognitive', 'post-traumatic stress', as well as 'tobacco', 'alcohol', and 'substance-related' disorders), and 3 final sections containing concluding questions, interviewer observations and interviewer ratings.

The instrument, designed for use by trained interviewers who are not clinicians, has shown high interrater $(k>0.70)$ (Cottler, Helzer, Mager, Spitznagel, \& Compton, 1991; Wittchen et al., 1991) and test-retest reliability (Reed, Gander, \& Pfister, 1998; Wittchen, 1994). However, the assessment of psychosis with CIDI by lay interviewers is not considered reliable (Anthony et al., 1985; Wittchen, 1994). Therefore, in the EDSP Study, trained and experienced clinical interviewers at the level of psychologist, who were allowed to probe with follow-up clinical questions, conducted the interviews in the respondents' homes. 
At baseline, the DIA-X/M-CIDI lifetime version was used. At each of the follow-up assessments, participants applied the interval version, which covers the period of assessment from the last interview until the next. However, data on the Gsection concerning psychosis and its clinical relevance were only collected at time point 2 (lifetime version) and time point 3 (interval version).

\section{Construction of SCL-Psychosis Subscale}

The 2 symptoms dimensions relevant for psychosis of the SCL-90-R, the so-called paranoid ideation and psychoticism subscales, were used to assess persistence of psychotic experiences over the period T0-T3. The 2 scales include self-reports on psychotic experiences which can be regarded, if not as clear-cut psychotic symptoms, as a subclinical expression of psychotic experiences. The 6-item paranoid ideation subscale represents paranoid behavior fundamentally as a disordered mode of thinking, including the cardinal characteristics of projective thought, hostility, suspiciousness, grandiosity, centrality and fear of loss of autonomy (items $8,18,43,68,76$, and 83 ). The 10-item psychoticism subscale was developed in a fashion to represent the construct as a continuous dimension of human experience, including items indicative of a withdrawn, isolated, schizoid lifestyle, and first-rank symptoms of schizophrenia, such as hallucinations and thought broadcasting (items 7, 16, 35, 68, 77, 84, 85, 87, 88, and 90). The severity scale of each symptom was scored from 'not-at-all' (0) to 'extremely' (4). The psychoticism and paranoia scales are strongly correlated (in the current sample, mean correlation over T0-T3 was .7) and the items in the scales have high predictive validity for future deficiency in social achievement and functioning (Rossler et al., 2007).

Therefore, conform previous research in this sample (Henquet et al., 2005; Spauwen et al., 2006), psychoticism and paranoia ideation subscales were combined into one Psychosis scale (hereafter: 'SCL-psychosis subscale') by summing their scores. The resulting SCL-psychosis subscale thus was a continuous score displaying a half-normal distribution at all time points (T0-T3). Previous research has shown that instruments assessing psychotic experiences dimensionally typically display a half-normal distribution (van Os et al., 2009).

\section{Assessment of Persistence of Psychotic Experiences over the Period T0-T2}

In order to examine the hypothesis relating to persistence over time, a discrete variable indicating presence or absence of psychosis across interview waves was, per definition, necessary. For the purpose of the analyses, a dichotomous psychosis variable was created using a cutoff point to define the group of individuals with the highest $10 \%$ of scores of the SCL-psychosis subscale (hereafter: 'psychosis expression'), consistent with previous analyses in this sample (Cougnard et al., 
2007; Henquet et al., 2005) and congruent with the meta-analytic rate for the prevalence of psychotic symptoms in the general population (van Os et al., 2009). Nevertheless, as a $10 \%$ cutoff remains arbitrary, sensitivity analyses were conducted using more and less conservative cutoff point values of the highest $5 \%$ and the highest $15 \%$ of the SCL-psychosis subscale.

A variable was defined indicating the degree of persistence of psychotic experiences over the period T0-T2. This resulted in a summary score variable consisting of 4 levels of psychosis expression: never (0), once (sporadic) (1), twice (recurrent) (2), and thrice (persistent) (3). This variable will hereafter be referred to as 'psychosis persistence'.

\section{Assessment of Clinical Relevance of Psychotic Experiences at T2 and T3}

Clinical relevance related to psychotic experiences was assessed by interview ratings from the DIA-X/M-CIDI in terms of psychotic impairment, representing the first step that needs explaining and predicting in tracking the ontogenesis of psychotic disorder in the general population. In order to define psychotic impairment, secondary dysfunction and help-seeking behavior was only examined in individuals with evidence of DIA-X/M-CIDI-positive psychotic symptoms at T2 (lifetime) and T3 (interval version).

Presence of positive psychotic experiences was broadly defined as any rating of "present" on any of the 20 DIA-X/M-CIDI core psychosis items (G1, G2a, G3-G5, G7-G13, G13b, G14, G17, G18, G20, G20C, G21, and G22a), including 14 delusion items, 5 hallucination items and 1 item on passivity phenomena. Items relate to classic psychotic symptoms involving, eg, persecution, thought interference, auditory hallucinations, and passivity phenomena. Participants were first invited by the psychologist to read a list of all the psychotic experiences and then asked whether they ever experienced such symptoms (list and phrasing available upon request). All these psychosis items were rated in 2 ways: absent (1) and present (5); all the psychosis DIA-X/M-CIDI items used in the present study were coded in a dichotomous manner without intermediate levels.

\section{Help-seeking}

Help-seeking behavior secondary to psychotic experiences was assessed using 3 DIA-X/M-CIDI items. Two psychosis section items were used: G16 (delusions) and G23 (hallucinations), which were phrased as follows: "Did you tell a doctor about ... (the psychosis section beliefs/experiences previously acknowledged by the participant along with a visual representation from the response booklet) you have had?" A third item from the concluding section was added (Q1DG); participants were shown a list on which several types of outpatient or inpatient institutions for 
mental health problems were mentioned, ranging from general practitioner or school psychologist to psychiatric sheltered housing, and asked whether they had ever sought help at any of these institutions because of psychotic symptoms as elicited in the DIA-X/M-CIDI G section. The 3 items were rated in a dichotomous manner: no (1) and yes (5). Using these 3 help-seeking behavior items, a dichotomous variable 'help-seeking' was constructed, indicating a positive answer on any of the 3 questions (value label: 1) vs negative answers on all 3 questions (value label: 0).

\section{Dysfunction}

The dysfunction score of the psychosis DIA-X/M-CIDI section assessed the effect of the psychotic experiences on (1) feeling upset, unable to work, go to places, or enjoy oneself, at the time of having these experiences (item G28), (2) being less able to work since these experiences began (item G29), (3) being less able to make friends or enjoy social relationships since these experiences began (item G29a), and (4) how much their life and everyday activities were impaired when these experiences were at their worst (item G36). These 4 items were rated in a dichotomous manner: no (1) and yes (5). A dichotomous variable 'dysfunction' was dichotomously constructed, representing a positive answer on any of the 4 questions (value label: 1) vs negative answers on all 4 questions (value label: 0 ).

\section{The Outcome Measure: Psychotic Impairment}

Clinically relevant psychosis was finally defined in terms of 'psychotic impairment', a variable created as a combined outcome of help-seeking and dysfunction. This was rated as " 0 " in subjects with psychotic experiences who scored " 0 " on both help-seeking and dysfunction, and "1" in subjects scoring " 1 " on either or both of help-seeking and dysfunction.

\section{Caseness}

In order to validate the outcome measure Psychotic Impairment in terms of clinical relevance, the X16 DIA-X/M-CIDI item was used. This item, rating the interviewer's opinion regarding clinical evidence of psychological ill health, consists of 4 levels: essentially not noticeable (0), not very noticeable (1), clearly ill (2), and very ill (3). The dichotomous variable 'caseness' was defined as any score above 1, thus indicating individuals with a noticeable level of psychiatric caseness.

\section{Risk set}

All the analyses were conducted in the younger cohort who (1) had been examined in the 4 waves of the study, (2) had completed the psychoticism and paranoia SCL90-R subscales at T0-T3, and (3) had completed the DIA-X/M-CIDI psychosis G 
section at T2 and T3 $(n=845)$. Of the 1395 individuals in the younger cohort at baseline, 845 fulfilled the risk set criteria.

Of the 1022 participants belonging to the younger cohort that completed the 4 waves, 94 individuals did not complete the self-report SCL-90-R at T1, 65 at T2 and 13 at T3. In addition, the G section of the DIA-X/M-CIDI was not available for 5 participants at $\mathrm{T} 2$. This resulted in a risk set of 845 participants who were included in the analysis.

Thus, a risk set of 845 participants remained from the total number of 1395 participants that composed the younger cohort at baseline. Of the 550 individuals not included in the risk set (hereafter: 'non-included group'), the reason was loss-tofollow up over the 8-year period in 373 and partial non-response in 177.

\section{Statistical Analyses}

All analyses were conducted using the software package STATA, version 9.2 (Stata Corporation, College Station: TX, USA. 2006).

In order to examine representativeness, the risk set $(n=845)$ was compared to the non-included group $(n=550)$ in terms of sociodemographic characteristics such as age, gender, level of education, social status, and urban residence.

The focus of the study was on psychosis persistence in adolescents over a period of approximately 3.5 years (T0-T2), integrating the scores of 3 waves (T0, $\mathrm{T} 1$ and T2), and analyzing the influence of the degree of persistence on the onset of psychotic impairment at T3 (Figure 1). To this end, logistic regression analyses were performed to examine the associations between T0-T2 psychosis persistence on the one hand and T3 psychotic impairment on the other. Associations were expressed as odds ratios (ORs) and their 95\% confidence intervals (CIs). In order to ensure that the analyses focussed on true new onsets of psychotic impairment at T3, any individual with psychotic impairment assessed lifetime at T2 was excluded.

In order to calculate posttest probabilities (PP) for T0-T2 psychosis persistence in relation to the $\mathrm{T} 3$ outcome of psychotic impairment, excluding any individual with lifetime T2 psychotic impairment, the STATA DIAGTEST procedure was used.

For both the logistic regression analyses and the calculation of PPs, a further check was introduced by additionally excluding individuals reporting at T2 that they had received any professional help for any psychological problem (DIA-X/MCIDI Q1 item). 
In addition, sensitivity analyses were conducted using more and less conservative cut-off point values of the highest $5 \%$ and the highest $15 \%$ of the SCL-psychosis subscale. Logistic regression was used to validate the T3 outcome measure psychosis impairment against the $\mathrm{T} 3$ caseness variable.

\section{Results}

\section{Subject Characteristics}

The average age of the risk set at T0 was 15.08 years (range: $14-17 ; \mathrm{SD}=1.1$ ). Of all participants, 444 were boys (52.5\%) and 401 girls (47.5\%). Other demographic characteristics are depicted in Table 1.

In comparison with the risk set $(\mathrm{n}=845)$, individuals from the nonincluded group $(\mathrm{n}=550)$ were of similar age (mean age $=15.18$ years), gender distribution (boys: $49.1 \%)$, or urban residence $(69.1 \%$ vs $72 \%$ for included vs nonincluded, respectively). The nonincluded group had a lower level of education (included lower level: $9.7 \%$ vs nonincluded lower level: $\left.28.6 \% ; \chi^{2}=100.41, p=.000\right)$ and lower social status (included lower status: $3.4 \%$ vs nonincluded lower status: $6.6 \%$; $\chi 2=12.36, \mathrm{p}=.006$ ).

\section{Occurrence of Psychotic Experiences}

The mean score of the continuous SCL-psychosis subscale was $21.5(\mathrm{SD}=5.6)$ at $\mathrm{T} 0,19.5(\mathrm{SD}=4.9)$ at $\mathrm{T} 1,19.5(\mathrm{SD}=4.7)$ at $\mathrm{T} 2$, and $18.7(\mathrm{SD}=4.7)$ at $\mathrm{T} 3$. With the interview ratings of the DIA-X/M-CIDI, the lifetime prevalence of psychotic symptoms at $\mathrm{T} 2$ was $21.8 \%(\mathrm{n}=184)$, and the interval cumulative incidence of psychotic symptoms at $\mathrm{T} 3$ was $12.7 \%(\mathrm{n}=107)$.

The distribution of the SCL-90-R psychosis persistence variable over T0-T2 was: never: $78.9 \%(n=666)$, once (sporadic): $15.6 \%(n=132)$, twice (recurrent): $3.9 \%(n=33)$, and thrice (persistent): $1.7 \%(n=14)$. Of the 83 individuals with psychosis expression at T0, $32(38.6 \%)$ again had psychosis expression at either T1 or T2. Of the 72 individuals with psychosis expression at T1, 29 (40.3\%) had psychosis expression at T2. Based on the DIA-X/M-CIDI interview ratings, of the $184(21.8 \%)$ individuals with any DIA-X/M-CIDI psychotic symptom at T2, 51 (27.7\%) again had evidence of psychotic symptoms at DIA-X/M-CIDI interview at T3. 
Table 1. Characteristics of Subjects at $\mathrm{T} 0$

\begin{tabular}{|c|c|c|}
\hline T0 $(n=845)$ & $n$ & $\%$ \\
\hline \multicolumn{3}{|l|}{ Gender } \\
\hline Male & 444 & 52.5 \\
\hline Female & 401 & 47.5 \\
\hline \multicolumn{3}{|c|}{ Level of education } \\
\hline Low & 82 & 9.7 \\
\hline Medium & 223 & 26.4 \\
\hline High & 540 & 63.9 \\
\hline \multicolumn{3}{|l|}{ Social status } \\
\hline Lower & 29 & 3.4 \\
\hline Middle & 516 & 61.1 \\
\hline Upper & 282 & 33.4 \\
\hline Other & 18 & 2.1 \\
\hline \multicolumn{3}{|l|}{ Residence } \\
\hline Rural & 261 & 30.9 \\
\hline Urban & 584 & 69.1 \\
\hline
\end{tabular}

Note: Level of education: low (mandatory basic school or learning a profession), medium (high school) and high (high school preparing for university and university). The participants were asked which level of education they were attending or, in the case of discontinuation, which was the highest level they had attended (demographic section, item A3 of the Munich-Composite International Diagnostic Interview [DIA-X/MCIDI]). Social status: lower (lower class, lower middle class), middle (middle middle class), upper (higher middle class, upper class), other (none of the above and missing values). The participants were asked to choose from specified options, which class he or she believed to be in (demographic section, item A16 of the DIA-X/M-CIDI). Residence: urban (the German city of Munich) and rural (the surrounding areas of Munich), obtained through population registries. The population density of the Munich surroundings areas was 553 persons per square mile and that of the city 4061 persons per square mile.

\section{Occurrence of Psychotic Impairment}

Of the 184 individuals with lifetime presence of DIA-X/M-CIDI psychotic symptoms at T2, 64 (34.8\%) had evidence of psychotic impairment. Of the 107 individuals with interval presence of DIA-X/M-CIDI psychotic symptoms at T3, 45 (42.1\%) had evidence of psychotic impairment.

Of the 64 individuals with psychotic impairment at T2, 11 (17.2\%) had again evidence of psychotic impairment at $\mathrm{T} 3$.

\section{Proportion of T3 Psychotic Impairment with Prior T0-T2 Psychosis Expression}

In terms of attributable risk, of the 47 individuals who displayed T3 psychotic impairment, 18 (38.3\%) had evidence of psychosis expression at any time point between T0-T2, of which $9(19.6 \%)$ more than once. 


\section{Transition to T3 Psychotic Impairment}

There was a strong association between $\mathrm{T} 3$ psychotic impairment and the T3 clinical caseness variable $(\mathrm{OR}=9.4,95 \% \mathrm{CI}: 3.1-28.6)$.

The absolute rate and relative risk of onset of $\mathrm{T} 3$ psychotic impairment as a function of the degree of T0-T2 psychosis persistence are depicted in Table 2. Excluding those with lifetime evidence of psychotic impairment at T2, the rates of T3 transition to psychotic impairment at the 4 levels of psychosis persistence (from 0 to 3$)$ were $3.7 \%(\mathrm{n}=23), 5.3 \%(\mathrm{n}=6), 16.0 \%(\mathrm{n}=4)$, and $27.3 \%(\mathrm{n}=3)$, yielding ORs (levels $1-3$ relative to 0 ) of 1.5 (95\%CI: $0.6-3.7 ; \mathrm{p}=.414), 5.0$ (95\%CI: $1.6-$ 15.9; $\mathrm{p}=.006)$ and $9.9(95 \% \mathrm{CI}: 2.5-39.8 ; \mathrm{p}=.001)$, respectively. Additional exclusion of those with $\mathrm{T} 2$ receipt of professional help for mental ill health similarly did not change these results (ORs of 1.1 [95\%CI: 0.4-3.2; $\mathrm{p}=.893$ ], 3.1 [95\%CI: 0.7-14.0; $\mathrm{p}=.151$ ] and 16.5 [95\%CI: 3.7-73.6; $\mathrm{p}=.000$ ], respectively).

Sensitivity analyses, using more and less conservative cutoff values for the SCL-psychosis subscale, showed the same pattern of increase in the rates of T3 transition to psychotic impairment. Thus, for the more conservative 5\% cutoff, risk of transition increased from the sporadic $(\mathrm{OR}=2.8 ; 95 \% \mathrm{CI}: 1.1-7.1)$ to the persistence level $(\mathrm{OR}=12.6 ; 95 \% \mathrm{CI}: 1.1-143.2)$. For the less conservative $15 \%$ cutoff, risk for transition increased similarly from the sporadic $(\mathrm{OR}=1.6 ; 95 \% \mathrm{CI}$ : 0.7-3.7) to the persistence level (OR=7.5; 95\%CI: 2.3-24.8).

The PPs for T0-T2 psychosis persistence in relation to T3 psychotic impairment, excluding individuals with lifetime evidence of $\mathrm{T} 2$ psychotic impairment, increased progressively from the sporadic (PP:5.3\%; CI: 3.7\%-6.9\%) to the persistence level (PP:27.3\%; CI: 23.8\%-30.7\%) (Table 2). Predictive values were even higher when the risk set was additionally restricted to individuals with no $\mathrm{T} 2$ receipt of professional help for mental health problems (Table 2). 







\section{Discussion}

\section{Findings}

This is the first study examining the influence of persistence of subclinical psychotic experiences in a sample of adolescents and young adults in the general population in relation to the transition to clinically relevant psychosis. The results showed, first, that subclinical psychosis was confirmed to be common (psychosis expression rates of $22 \%$ ) and mostly transitory, recurrence or persistence occurring in $30-40 \%$ of cases. Second, the findings demonstrated that persistent positive symptoms at a subclinical level pose a significant risk and can be considered a marker of emerging clinical states. A dose-response relationship was found: the longer the period over which subclinical psychosis remained abnormally nontransitory, the greater the risk of developing associated impairment. Third, although most subclinical psychosis was transitory, these results showed that the impact of the relationship between the clinical and the subclinical was not negligible: Around two-fifths of new onset, clinically relevant psychosis in young adults could be traced to the subclinical psychosis phenotype expressed up to 8 years earlier, and one-fifth had displayed evidence of a recurrent or persistent subclinical phenotype. For some individuals, psychosis is thus the denouement of a long process of subclinical experiences and functional deficits. Consequently, these findings provide evidence for the continuity over time from subclinical persistent psychotic experiences to clinical psychotic states, yielding insight into the dynamics preceding the clinical onset of psychotic disorder from a population perspective.

\section{Persistence of Subclinical Psychosis Expression}

The recurrence-persistence rate of subclinical psychosis in this study was higher than reported in previous population-based studies. In a 2-year follow-up of a general population sample aged 18-64 years, Hanssen et al (M. Hanssen et al., 2005 ) found a 2 -year $8 \%$ persistence rate of baseline subclinical psychosis, and an $8 \%$ transition rate from subclinical to clinical psychosis. In the present study, the recurrence-persistence rate was $30-40 \%$ over time, considerably higher. The most likely factor to explain this difference is the much younger age of the current sample. Given the rapid decline of psychosis expression with age (Peters et al., 1999; van Os et al., 2000; Verdoux et al., 1998), continuity over time will become less likely with advancing age. Thus, in a previous follow-up study of adolescents, a 3 -year persistence rate was found of $40 \%$, similar to that of the current study (Escher, Romme, Buiks, Delespaul, \& Van Os, 2002). 


\section{The Persistent Subclinical Psychosis Pathway into Clinical Psychosis}

Although the development of subclinical psychotic symptoms is an initial step to the emergence of psychotic disorder in a significant proportion of cases, not more than two-fifths of all psychotic impairment could be attributed to this pathway. This likely represents an underestimate, however, caused in part by the uneven sampling intervals from $\mathrm{T} 0$ to $\mathrm{T} 3$. Thus, the relatively wide sampling interval between $\mathrm{T} 2$ and T3 (4.9 y on average) may have resulted in participants underreporting experiences and instances of help-seeking. Similarly, the SCL-90-R assesses symptoms over the last two weeks, thus creating the possibility of underreporting psychotic experiences over the period T0-T2. In addition, other domains of psychopathology, such as depression and anxiety, may also contribute to prediction and attributable risk and need to be investigated further (van Os \& Delespaul, 2005). Furthermore, more work is needed on the role of cognitive alterations in the ontogenesis of psychotic states, given the fact that meta-analytic work indicates that this domain is orthogonal to the positive and affective symptoms of psychosis (Dominguez, Viechtbauer, Simons, van Os, \& Krabbendam, 2009), yet is likely to impact on the domain of functional impairment in the onset of clinical psychotic states.

Even if it represents a likely underestimation, the fact that $40 \%$ of new onset, clinically relevant psychosis can be traced to the early subclinical psychosis phenotype in the general population sheds light on the ontogenesis of psychotic states in the general population. However, much more work is needed in order to understand the process resulting in clinical need in individuals at risk, and the current findings need to be replicated before firm conclusions can be drawn.

\section{From Persistent Subclinical Psychosis to Impairment: Possible Mechanisms}

The positive psychosis pathway may be understood as the phenomenological correlate of the classical dopamine (DA) hypothesis which proposes that hyperactivity of DA transmission from DA cell bodies located in the ventral tegmental area of the midbrain to their terminal fields in the nucleus accumbens and limbic cortex results in the development of positive psychotic symptoms (Laruelle, 2000; Lieberman, Sheitman, \& Kinon, 1997). DAergic hyperactivity may be associated with what has been called a state of endogenous sensitization (Laruelle, 2000). The liability for psychosis may involve deficits in neural regulation resulting in a pathological condition of neurochemical sensitization of the mesolimbic DAergic system analogous to the preclinical model of behavioral sensitization induced by administration of DA-releasing drugs (Lieberman et al., 1997). Behavioral sensitization is a progressive, enduring enhancement of behaviors that develops following repeated stimulant administration. It is mediated in part by DAergic pathways that are also thought to modulate the development of psychosis (Wolf, White, Nassar, Brooderson, \& Khansa, 1993). 
Endogenous neurochemical sensitization may arise in part as a result of exposure to environmental conditions (Laruelle, Kegeles, \& Abi-Dargham, 2003). For example, environmental stress can induce sensitization and has been demonstrated to be cross-reactive with many types of pharmacologically induced sensitization, including DA and opiate agonists (Lieberman et al., 1997). It has been suggested that several environmental risk factors, eg, perinatal injury, childhood trauma, repeated life events and drug abuse, may contribute to DA sensitization during development (Murray, McDonald, \& Bramon, 2002). It is attractive to speculate that a genetic tendency to abnormal DA sensitization is associated with the tendency to express low-grade psychotic experiences that in most individuals will only be expressed transitorily over development. However, in the case of developmental exposure to environmental risk factors, individuals may develop a sensitized DA system resulting in abnormally persistent and, eventually, clinically relevant psychotic states (Cougnard et al., 2007).

\section{Methodological Issues}

First, the dynamic hypothesis of psychosis onset was examined in the context of a large-scale epidemiological study not specifically designed for this purpose. It was thus constrained by the specific psychometric tools and sampling frame.

Second, the group comparison between the risk set $(n=845)$ and the nonincluded group $(n=550)$ showed that the latter had a lower level of education and social status. Because these are risk factors for psychotic experiences (Johns \& van Os, 2001; van Os et al., 2000), attrition of these individuals may have led to an underestimation of psychosis outcomes in our sample. Bias of reported associations, however, would have occurred only in the unlikely event that high levels of psychosis persistence would be associated with lower rates of transition to clinical psychosis in this group.

Third, in order to measure persistence of psychotic experiences, the SCL-90-R was used. The fact that this instrument only covers the past 2 weeks may have led to an underestimation of the T0-T2 psychosis persistence measure. As it is difficult to see how this would result in a spurious association with interviewer-assessed psychotic symptoms and impairment 5 years later, any error resulting from underestimation would have likely been random. Similarly, to the degree that the SCL-90-R overestimates the rate of psychotic symptoms (because of the misunderstandings arising from self-report), any resulting error would likely also be random. Furthermore, the way the measure of psychosis persistence in the present study was constructed, only considering those individuals with the highest $10 \%$ of scores of the continuous SCL-psychosis subscale, prevented misclassification due 
to overestimation of such experiences on the basis of incidental misunderstanding of items.

Four, the DIA-X/M-CIDI was used to measure clinical relevance of psychotic experiences in terms of psychotic impairment, evaluating secondary dysfunction and help-seeking behavior. On the one hand, help-seeking may have led to an underestimation of true positives in the case of individuals who did not seek for help in spite of suffering from true clinical psychotic symptoms. On the other hand, dysfunction may have led to an overestimation of true positives in the case that individuals reported secondary dysfunction only based on subjective level of distress. Nonetheless, the validation of the outcome variable against the clinical judgment of illness strongly supports the validity of psychotic impairment as a proxy measure of clinical relevance.

Five, the number of cases with recurrence-persistence of psychosis expression that developed psychotic impairment was relatively small, conform the expected low rate in the general population, resulting in wide CIs.

Six, the main instruments to assess psychotic experiences and symptoms in the current study (SCL-90-R and DIA-X/M-CIDI) are essentially self-report. Although this is likely to have generated false-positive psychotic experiences, likely increasing random error as discussed earlier, there is nevertheless a substantial literature on the (predictive or other forms of) validity of self-reported psychotic symptoms, assessed with DIA-X/M-CIDI, SCL-90-R and other instruments (van Os et al., 2009). For example, one study validated self-reported psychotic symptoms against a clinical interview with the Brief Psychiatric Rating Scale (Overall \& Gorham, 1962) in a sample of psychotic patients, concluding that self-reported scores reflect clinical experience of psychosis (van Os et al., 1999). Poulton et al (Poulton et al., 2000) presented strong evidence for predictive validity of CIDI selfreported psychotic symptoms. Similarly, Hanssen et al (M. S. Hanssen, Bijl, Vollebergh, \& van Os, 2003) confirmed that the probability of having a psychotic disorder increased in a dose-response fashion with the level of self-reported psychotic experiences in the general population. In 2006, Konings et al (Konings, Bak, Hanssen, van Os, \& Krabbendam, 2006) confirmed the validity and reliability for the measurement of psychotic experiences with a self-reported instrument in the general population. A recent, 20-year longitudinal cohort study on psychotic symptoms using the SCL-90-R also showed high predictive validity of the SCL-90$\mathrm{R}$ psychosis items used in the current sample in terms of deficiencies in social achievement and functioning (Rossler et al., 2007).

Finally, the current study had a longitudinal, population-based epidemiological perspective, contrasting with the shorter term, selective, sample-enriching approach 
focusing on help-seekers in the ultrahigh-risk literature (van Os \& Delespaul, 2005). The two perspectives and their respective populations, instruments and outcomes are too different to make findings readily comparable.

\section{Funding}

This work is further part of the EDSP Study and is supported by the German Federal Ministry of Education and Research (BMBF) project no. 01EB9405/6, 01EB9910/6, EB10106200, 01EB0140, and 01EB0440. Part of the field work and analyses were also additionally supported by grants of the Deutsche Forschungsgemeinschaft (DFG) LA1148/1-1, WI2246/7-1, and WI709/8-1; European Community's Sixth Framework Programme (Marie Curie Early Stage Research Training Fellowship under contract number MEST-CT-2005-020589 to M.d.G.D.); Dutch Medical Council (VENI grant nr 916.76.147 to M.W).

\section{Acknowledgements}

Declarations of interest: None. Data Access and responsibility: Principal investigators of the EDSP Study are Dr Wittchen and Dr Lieb. Both take responsibility for the integrity of the study data. All authors and coauthors had full access to all study data. Data analysis and manuscript preparation were completed by the authors and coauthors of this article, who take responsibility for its accuracy and content. Core staff members of the EDSP group are Dr. Kirsten von Sydow, Dr. Gabriele Lachner, Dr. Axel Perkonigg and Dr. Peter Schuster -Dipl Stat; Michael Höfler, Holger Sonntag, Tanja Brückl, Elzbieta Garczynski, Dr. Barbara Isensee, Barbara Spiegel, Andrea Schreier, Dr. Ursula wunderlich, Dr. Petra Zimmermann, Datja Beesdo, and Antje Bitner -Dipl Psych; Agnes Nocon and Dr. Chris Nelson Dipl Inf; Hildegard Pfister and Dr. Victoria Reed -Dipl Soz. Scientific advisors are Dr. Jules Angst (Zurich), Dr. Jürgen Margraf (Basel), Dr. Günther Esser (Potsdam), Dr. Kathleen Merikangas (National Institute of Mental Health, Bethesda), Dr. Ron Kessler (Harvard, Boston) and Dr. van Os (Maastricht). Financial Disclosure: Dr. Lieb-speaker honoraria: Wyeth; Dr. Wittchen-research support: Eli Lilly and Company, Novartis, Pfizer, and Schering-Plough; consulting: Eli Lilly, GlaxoSmithKline Pharmaceuticals, Hoffmann-La Roche Pharmaceuticals, Novartis, Pfizer and Wyeth; speaker honoraria: Novartis, Schering-Plough, Pfizer and Wyeth. Dr. van Os-past/present unrestricted research support: Eli Lilly, GSK, Organon, BMS and AstraZeneca; independent speaker honoraria: Eli Lilly, BMS, Lundbeck, Organon, Janssen-Cilag and AstraZeneca. 


\section{References}

Akiskal, H. S., \& Vazquez, G. H. (2006). [Widening the borders of the bipolar disorder: validation of the concept of bipolar spectrum]. Vertex, 17(69), 340-346.

Angst, J., \& Cassano, G. (2005). The mood spectrum: improving the diagnosis of bipolar disorder. Bipolar Disord, 7 Suppl 4, 4-12.

Anthony, J. C., Folstein, M., Romanoski, A. J., Von Korff, M. R., Nestadt, G. R., Chahal, R., et al. (1985). Comparison of the lay Diagnostic Interview Schedule and a standardized psychiatric diagnosis. Experience in eastern Baltimore. Arch Gen Psychiatry, 42(7), 667-675.

Chapman, L. J., Chapman, J. P., Kwapil, T. R., Eckblad, M., \& Zinser, M. C. (1994). Putatively psychosisprone subjects 10 years later. J Abnorm Psychol, 103(2), 171-183.

Constantino, J. N., \& Todd, R. D. (2003). Autistic traits in the general population: a twin study. Arch Gen Psychiatry, 60(5), 524-530.

Cottler, L. B., Helzer, J. E., Mager, D., Spitznagel, E. L., \& Compton, W. M. (1991). Agreement between DSM-III and III-R substance use disorders. Drug Alcohol Depend, 29(1), 17-25.

Cougnard, A., Marcelis, M., Myin-Germeys, I., R, D. E. G., Vollebergh, W., Krabbendam, L., et al. (2007). Does normal developmental expression of psychosis combine with environmental risk to cause persistence of psychosis? A psychosis proneness-persistence model. Psychol Med, 37(4), 513-527.

Derogatis, L. R., \& Cleary, P. A. (1977). Confirmation of the dimensional structure of the SCL-90: a study in construct validation. Journal of Clinical Psychology, 33, 981-989.

Dominguez, M. D. G., Viechtbauer, W., Simons, C. J. P., van Os, J., \& Krabbendam, L. (2009). Are Psychotic Psychopathology and Neurocognition Orthogonal? A systematic Review of their Associations. Psychol Bull, 135(1), 157-171.

Escher, S., Romme, M., Buiks, A., Delespaul, P., \& van Os, J. (2002). Independent course of childhood auditory hallucinations: a sequential 3-year follow-up study. Br J Psychiatry Suppl, 43, s10-18.

Fergusson, D. M., Horwood, L. J., \& Swain-Campbell, N. R. (2003). Cannabis dependence and psychotic symptoms in young people. Psychol Med, 33(1), 15-21.

Hanssen, M., Bak, M., Bijl, R., Vollebergh, W., \& van Os, J. (2005). The incidence and outcome of subclinical psychotic experiences in the general population. Br J Clin Psychol, 44(Pt 2), 181-191.

Hanssen, M. S., Bijl, R. V., Vollebergh, W., \& van Os, J. (2003). Self-reported psychotic experiences in the general population: a valid screening tool for DSM-III-R psychotic disorders? Acta Psychiatr Scand, 107(5), 369-377.

Henquet, C., Krabbendam, L., Spauwen, J., Kaplan, C., Lieb, R., Wittchen, H. U., et al. (2005). Prospective cohort study of cannabis use, predisposition for psychosis, and psychotic symptoms in young people. Bmj, 330(7481), 11.

Johns, L. C., \& van Os, J. (2001). The continuity of psychotic experiences in the general population. Clin Psychol Rev, 21(8), 1125-1141.

Judd, L. L., \& Akiskal, H. S. (2003). The prevalence and disability of bipolar spectrum disorders in the US population: re-analysis of the ECA database taking into account subthreshold cases. J Affect Disord, 73(1-2), 123-131.

Kendler, K. S., \& Diehl, S. R. (1993). The genetics of schizophrenia: a current, genetic-epidemiologic perspective. Schizophr Bull, 19(2), 261-285.

Konings, M., Bak, M., Hanssen, M., van Os, J., \& Krabbendam, L. (2006). Validity and reliability of the CAPE: a self-report instrument for the measurement of psychotic experiences in the general population. Acta Psychiatr Scand, 114(1), 55-61.

Krabbendam, L., Myin-Germeys, I., Hanssen, M., \& van Os, J. (2005). Familial covariation of the subclinical psychosis phenotype and verbal fluency in the general population. Schizophr Res, 74(1), 37-41.

Laruelle, M. (2000). The role of endogenous sensitization in the pathophysiology of schizophrenia: implications from recent brain imaging studies. Brain Res Brain Res Rev, 31(2-3), 371-384.

Laruelle, M., Kegeles, L. S., \& Abi-Dargham, A. (2003). Glutamate, dopamine, and schizophrenia: from pathophysiology to treatment. Ann N Y Acad Sci, 1003, 138-158.

Lieb, R., Isensee, B., von Sydow, K., \& Wittchen, H. U. (2000). The Early Developmental Stages of Psychopathology Study (EDSP): a methodological update. Eur Addict Res, 6(4), 170-182. 
Lieberman, J. A., Sheitman, B. B., \& Kinon, B. J. (1997). Neurochemical sensitization in the pathophysiology of schizophrenia: deficits and dysfunction in neuronal regulation and plasticity. Neuropsychopharmacology, 17(4), 205-229.

Murray, R. M., McDonald, C., \& Bramon, E. (2002). Neurodevelopmental impairment, dopamine sensitisation, and social adversity in schizophrenia. World Psychiatry, 1(3), 137-145.

Overall, J. E., \& Gorham, D. R. (1962). The brief psychiatric rating scale. Psychol Rep, 10, 799-812.

Perala, J., Suvisaari, J., Saarni, S. I., Kuoppasalmi, K., Isometsa, E., Pirkola, S., et al. (2007). Lifetime prevalence of psychotic and bipolar I disorders in a general population. Arch Gen Psychiatry, 64(1), 1928.

Peters, E. R., Joseph, S. A., \& Garety, P. A. (1999). Measurement of delusional ideation in the normal population: introducing the PDI (Peters et al. Delusions Inventory). Schizophr Bull, 25(3), 553-576.

Poulton, R., Caspi, A., Moffitt, T. E., Cannon, M., Murray, R., \& Harrington, H. (2000). Children's selfreported psychotic symptoms and adult schizophreniform disorder: a 15-year longitudinal study. Arch Gen Psychiatry, 57(11), 1053-1058.

Reed, V., Gander, F., \& Pfister, H. (1998). To what degree does the Composite International Diagnostic Interview (CID) correctly identify DSM-IV disorders? Testing validity issues in a clinical sample. . International Journal of Methods in Psychiatric Research, 7, 142-155.

Rossler, W., Riecher-Rossler, A., Angst, J., Murray, R., Gamma, A., Eich, D., et al. (2007). Psychotic experiences in the general population: a twenty-year prospective community study. Schizophr Res, 92(13), 1-14.

Spauwen, J., Krabbendam, L., Lieb, R., Wittchen, H. U., \& van Os, J. (2006). Evidence that the outcome of developmental expression of psychosis is worse for adolescents growing up in an urban environment. Psychol Med, 36(3), 407-415.

Stefanis, N. C., Hanssen, M., Smirnis, N. K., Avramopoulos, D. A., Evdokimidis, I. K., Stefanis, C. N., et al. (2002). Evidence that three dimensions of psychosis have a distribution in the general population. Psychol Med, 32(2), 347-358.

Stefanis, N. C., van Os, J., Avramopoulos, D., Smyrnis, N., Evdokimidis, I., Hantoumi, I., et al. (2004). Variation in catechol-o-methyltransferase val158 met genotype associated with schizotypy but not cognition: a population study in 543 young men. Biol Psychiatry, 56(7), 510-515.

van Os, J., \& Delespaul, P. (2005). Toward a world consensus on prevention of schizophrenia. Dialogues Clin Neurosci, 7(1), 53-67.

van Os, J., Hanssen, M., Bijl, R. V., \& Ravelli, A. (2000). Strauss (1969) revisited: a psychosis continuum in the general population? Schizophr Res, 45(1-2), 11-20.

van Os, J., Linscott, R. J., Myin-Germeys, I., Delespaul, P., \& Krabbendam, L. (2009). A systematic review and meta-analysis of the psychosis continuum: evidence for a psychosis proneness-persistenceimpairment model of psychotic disorder. Psychol Med, 39(2), 179-195.

van Os, J., Verdoux, H., Maurice-Tison, S., Gay, B., Liraud, F., Salamon, R., et al. (1999). Self-reported psychosis-like symptoms and the continuum of psychosis. Soc Psychiatry Psychiatr Epidemiol, 34(9), $459-463$.

Verdoux, H., van Os, J., Maurice-Tison, S., Gay, B., Salamon, R., \& Bourgeois, M. (1998). Is early adulthood a critical developmental stage for psychosis proneness? A survey of delusional ideation in normal subjects. Schizophr Res, 29(3), 247-254.

Voglmaier, M. M., Seidman, L. J., Niznikiewicz, M. A., Dickey, C. C., Shenton, M. E., \& McCarley, R. W. (2000). Verbal and nonverbal neuropsychological test performance in subjects with schizotypal personality disorder. Am J Psychiatry, 157(5), 787-793.

Wittchen, H. U. (1994). Reliability and validity studies of the WHO--Composite International Diagnostic Interview (CIDI): a critical review. J Psychiatr Res, 28(1), 57-84.

Wittchen, H. U., Perkonigg, A., Lachner, G., \& Nelson, C. B. (1998). Early developmental stages of psychopathology study (EDSP): objectives and design. Eur Addict Res, 4(1-2), 18-27.

Wittchen, H. U., \& Pfister, H. (1997). DIS-X-Interviews: Manual für Screening-Verfahren und Interview; Interviewheft Längsschnittuntersuchung (DIA-X-Lifetime); Ergänzungsheft (DIA-X-Lifetime); Interviewheft Querschnittsuntersuchung (DIA-X-12 Monats-Version); Ergänzungsheft (DIA-X-12 Monats-Version); PC-Programm zur Durchführung der Interviews (Längsund Querschnittsuntersuchung). Auswertungsprogramm. Frankfurt: Swets \& Zeitlinger. . 
Wittchen, H. U., Robins, L. N., Cottler, L. B., Sartorius, N., Burke, J. D., \& Regier, D. (1991). Cross-cultural feasibility, reliability and sources of variance of the Composite International Diagnostic Interview (CIDI). The Multicentre WHO/ADAMHA Field Trials. Br J Psychiatry, 159, 645-653, 658.

Wolf, M. E., White, F. J., Nassar, R., Brooderson, R. J., \& Khansa, M. R. (1993). Differential development of autoreceptor subsensitivity and enhanced dopamine release during amphetamine sensitization. J Pharmacol Exp Ther, 264(1), 249-255.

World Health Organization. (1990). Composite International Diagnostic Interview (CIDI), Version 1.0. Geneva: WHO.

Yung, A. R., Phillips, L. J., Yuen, H. P., Francey, S. M., McFarlane, C. A., Hallgren, M., et al. (2003). Psychosis prediction: 12-month follow up of a high-risk ("prodromal") group. Schizophr Res, 60(1), 2132. 


\title{
CHAPTER 4
}

\author{
AFFECTIVE DYSREGULATION AND \\ REALITY DISTORTION:A 10-YEAR \\ PROSPECTIVE STUDY OF THEIR \\ ASSOCIATION AND CLINICAL \\ RELEVANCE
}

Inge van Rossum ${ }^{1}$, Maria-de-Gracia Dominguez ${ }^{1}$, Roselind Lieb ${ }^{2,3}$, Hans-Ulrich Wittchen ${ }^{2,4}$, Jim van Os ${ }^{1,5}$

\footnotetext{
${ }^{1}$ Department of Psychiatry and Neuropsychology, South Limburg Mental Health Research and Teaching Network, EURON, Maastricht University Medical Centre, Maastricht, The Netherlands.

${ }^{2}$ Max Planck Institute of Psychiatry, Clinical Psychology and Epidemiology Unit, Munich, Germany. ${ }^{3}$ Epidemiology and Health Psychology, Institute of Psychology, University of Basel, Basel, Switzerland. ${ }^{4}$ Institute of Clinical Psychology and Psychotherapy, Technical University Dresden, Dresden, Germany. ${ }^{5}$ Division of Psychological Medicine, Institute of Psychiatry, London, UK.
}

Schizophrenia Bulletin, doi:10.1093/schbul/sbp101 


\begin{abstract}
Objective

Evidence from clinical patient populations indicates that affective dysregulation is strongly associated with reality distortion, suggesting that a process of misassignment of emotional salience may underlie this connection.
\end{abstract}

\title{
Method
}

To examine this in more detail without clinical confounds, affective regulationreality distortion relationships, and their clinical relevance, were examined in a German prospective cohort community study. A cohort of 2524 adolescents and young adults aged 14-24 years at baseline was examined by experienced psychologists. Presence of psychotic experiences and (hypo)manic and depressive symptoms was assessed at two time points (3.5 and up to 10 years after baseline) using the Munich-Composite International Diagnostic Interview. Associations were tested between level of affective dysregulation on the one hand and incidence of psychotic experiences, persistence of these experiences and psychotic impairment on the other.

\section{Results}

Most psychotic experiences occurred in a context of affective dysregulation, and bidirectional dose-response was apparent with greater level of both affective dysregulation and psychotic experiences. Persistence of psychotic experiences was progressively more likely with greater level of (hypo)manic symptoms (OR trend=1.51, $\mathrm{p}<0.001$ ) and depressive symptoms (OR trend=1.15, $\mathrm{p}=0.012$ ). Similarly, psychotic experiences of clinical relevance were progressively more likely to occur with greater level of affective dysregulation (depressive symptoms: OR trend=1.28, $\mathrm{p}=0.002$; (hypo)manic symptoms: OR trend=1.37, $\mathrm{p}=0.036$ ).

\section{Conclusions}

Correlated genetic liabilities underlying affective and non-affective psychotic syndromes may be expressed as correlated dimensions in the general population. Also, affective dysregulation may contribute causally to the persistence and clinical relevance of reality distortion, possibly by facilitating a mechanism of aberrant salience attribution.

\section{Key words}

Epidemiology, Adolescent, Psychosis, Affective Symptoms 


\section{Introduction}

Evidence from multiple domains indicates that affective dysregulation is strongly associated with reality distortion (Garety, Bebbington, Fowler, D, \& Kuipers, 2007; Myin-Germeys \& van Os, 2007). Genetic epidemiological studies have demonstrated that the liabilities for bipolar disorder and schizophrenia are correlated (Cardno, Rijsdijk, Sham, Murray, \& McGuffin, 2002; Craddock \& Owen, 2007; Lichtenstein, Yip, \& Bjork, 2009). Psychopathological studies have demonstrated that psychotic experiences are reported within the context of a range of affective clinical disorders (Dunayevich \& Keck, 2000; Sareen, Cox, Goodwin, $\&$ Asmundson, 2005) and, conversely, high rates of affective symptoms have been demonstrated in patients diagnosed with DSM-IV non-affective psychotic disorders (Peralta \& Cuesta, 2009; Zisook et al., 1999) and schizotypy (Debbane, van der Linden, Gex-Fabry, \& Eliez, 2009). Specific aspects of delusional content and severity of psychotic experiences are associated with dysfunctional emotional processes (Bentall, Rowse, \& Shryane, 2009; Green, Garety, \& Freeman, 2006; Smith, Fowler, \& Freeman, 2006). Although most of the work in this area is crosssectional and conducted in clinical samples, making it difficult to disentangle the direction of effects between affective and cognitive processes, and to distinguish between illness cause and illness consequence, the strong and consistent associations between affective states and reality distortion may imply causality.

Experimental work by Holt and colleagues (Holt et al., 2006) indicates that patients with a diagnosis of schizophrenia who had delusions were more likely to assign (negative) affective meanings to neutral stimuli compared to those without delusional ideation. The magnitude of this response bias correlated with the severity of delusions. The findings were interpreted as consistent with an inappropriate activation of a stimulus-independent internal 'salience detector', leading to misassignment of emotional salience to neutral, ambiguous stimuli and ultimately to the formation of delusions (Holt et al., 2006). Thus, affective dysregulation may result in maladaptive appraisal patterns of events, triggering a search for an explanation of their meaning that in turn increases the risk for positive psychotic experiences in vulnerable individuals (Garety, Kuipers, Fowler, Freeman, \& Bebbington, 2001). Dysregulation in dopamine transmission, facilitating stimulusindependent release of dopamine, causing aberrant assignment of salience and motivational significance to external objects (leading to delusions) and internal representations of percepts and memories (leading to hallucinations) may represent the underlying neurobiological vulnerability mediating the process of reality distortion (Kapur, 2003).

Research on associations between variable clinical characteristics such as affective symptoms and psychotic experiences in clinical samples cannot examine 
to what degree such associations may arise as a result of the illness itself, and how interacting affective and cognitive processes, from a perspective of risk, may contribute to the onset of need for care and patient status. Finally, to the degree that the affective and cognitive processes in patients with psychotic disorder are universal, that is, are quantitative variations of normal human mentation (van Os \& Kapur, 2009), more fundamental knowledge is needed on their association in the general population, as otherwise a correct interpretation of their role in pathological states such as psychotic disorder is not possible.

It has long been recognized that schizophrenia-related pathology is also expressed, at attenuated levels, in individuals with 'schizotypal' or 'schizoid' personality traits. Systematic review of general population surveys indicates that the experiences associated with schizophrenia and related categories, such as paranoid delusional thinking and auditory hallucinations, are observed, in an attenuated form, in 5-8\% of healthy people (van Os, Linscott, Myin-Germeys, Delespaul, \& Krabbendam, 2009). These attenuated expressions may be conceived as the behavioral expression of the underlying distributed liability for schizophrenia and related disorders, just as higher levels of blood pressure express higher liability for cardiovascular disease in a dose-response fashion. This interpretation is validated by longitudinal research showing a link between psychotic experiences in the general population and later outcomes of psychotic disorder. Since the seminal study by Chapman and colleagues (Chapman, Chapman, Kwapil, \& Zinser, 1994), there is replicatory evidence from 2 birth cohorts (Poulton et al., 2000; Welham, Scott, \& Williams, 2008) and 3 representative general population cohorts (Dominguez, Wichers, Lieb, Wittchen, \& van Os, 2009; Hanssen, Bak, Bijl, Vollebergh, \& van Os, 2005; Werbeloff, Drukker, \& Dohrenwend, 2009) that low grade psychotic experiences such as delusional thinking and mild hallucinatory experiences may precede the diagnosis of psychotic disorder, including clinical diagnosis of schizophrenia requiring hospital admission by many years. It has been shown that particularly persistence of subclinical psychotic experiences over time is associated with increased risk of later transition to clinically relevant psychosis (Cougnard, Marcelis, \& Myin-Germeys, 2007; Dominguez et al., 2009). Additional evidence is provided by a body of work on help-seeking individuals with low-grade psychotic experiences who, when followed over time, display high conversion rates to clinical psychotic disorder (Cannon, Cornblatt, \& McGorry, 2007).

Therefore, epidemiological research in the general population can be useful in complementing clinical research on the link between affective dysregulation, emotional salience misattribution and psychosis, particularly when a longitudinal perspective can be added in order to clarify the direction of effects and to study the relationship with onset of impairment. Earlier general population surveys in Greece and the Netherlands have shown a high degree of overlap between psychotic 
experiences and affective symptoms below the threshold for clinical disorder (Kaymaz et al., 2007; Krabbendam, Myin-Germeys, Bak, \& van Os, 2005; Krabbendam, Myin-Germeys, Hanssen et al., 2005; Krabbendam \& van Os, 2005; Stefanis et al., 2002). For the current study, it was hypothesized that (i) level of affective dysregulation, in the form of depression or (hypo)mania symptoms (regardless of the presence of formal mood disorder) in the general population, would be strongly and linearly associated with experience of reality distortion, expressed as psychotic experiences (ii) presence of affective dysregulation would be associated with persistence of reality distortion over time and (iii) affective dysregulation would be associated with psychotic impairment in the context of reality distortion.

\section{Methods}

\section{Study Design and Population}

The Early Developmental Stages of Psychopathology (EDSP) study is a prospective-longitudinal cohort community study which collected data on the prevalence, incidence, risk factors, comorbidity and course of mental disorders. Following ethics committee approval, a representative population sample was randomly drawn from the 1994 German government population registers. The sample consisted of adolescents and young adults living in the Munich area aged 14-24 years at baseline. Because the primary goal of the study was to examine the incidence and developmental risk factors for psychopathology, stratification of the sample was performed by sampling 14 and 15 -year olds, presumed to have the highest incidence density, at twice the rate of 16 to 21 -year olds, and by sampling 22 to 24 -year olds at half this rate. The 4,809 sampled individuals were approached through letter and phone. Of these, 4263 were located and determined to be eligible for the study. Most interviews took place at the participant's home.The study consisted of a baseline survey (T0, $\mathrm{n}=3021)$ and 3 follow-up investigations (T1, T2 and T3), covering a time period of approximately 1.6 years $(\mathrm{T} 0-\mathrm{T} 1, \mathrm{SD}=0.2), 3.5$ years $(\mathrm{T} 0-\mathrm{T} 2, \mathrm{SD}=0.3)$ and 8.6 years $(\mathrm{T} 0-\mathrm{T} 3$, range $7.4-10.6$ years, $\mathrm{SD}=0.7$ ) respectively. Written informed consent was obtained from all participants. The study design and sample has been described in detail in previous reports (Lieb, Isensee, Von Sydow, \& Wittchen, 2000; Wittchen, Perkonigg, Lachner, \& Nelson, 1998).

\section{Instruments}

Interviews were conducted using the Computer-Assisted Personal Interview (CAPI) version of the Munich-Composite International Diagnostic Interview (DIA-X/MCIDI (Wittchen \& Pfister, 1997)), an updated version of the World Health 
Organization's CIDI version 1.2.The DIA-X/M-CIDI is a comprehensive, fully standardized diagnostic interview and assesses symptoms, syndromes and diagnoses of various mental disorders in accordance with the definitions and criteria of DSMIV, along with information about onset, duration, severity and psychosocial impairment. The CIDI is divided into 16 sections: a socio-demographic section, 12 sections consisting of 288 symptom questions regarding groups of mental disorders and three final sections containing concluding questions, interviewer observations and interviewer ratings. High validity (Reed et al., 1998) as well as high interrater and test-retest reliability of the CIDI have been established (Wittchen, 1994; Wittchen et al., 1991). In order to ensure reliability of the assessments, fully trained and experienced clinical psychologists who were allowed to probe with clinical follow-up questions conducted the interviews. At baseline, the lifetime version of the DIA-X/M-CIDI was used; for the follow-up interviews, the DIA-X/M-CIDI interval version was used, covering the respective time periods between interviews. As data on the DIA-X/M-CIDI G-section concerning psychosis and its clinical relevance were only collected at T2 (lifetime version, assessing lifetime cumulative incidence up to T2) and T3 (interval version, assessing onset of new, incident, symptoms or interval rate of any symptom between $\mathrm{T} 2$ and $\mathrm{T} 3$ ), the current analyses are limited to T2 and T3. The response rate was $84 \%$ at T2 $(n=2548)$ and $73 \%$ at $\mathrm{T} 3(\mathrm{n}=2210)$, covering an interval period of 4.9 years on average $(\mathrm{SD}=0.6)$.

\section{Assessment of Reality Distortion}

Information from the CIDI psychosis section and the clinical interview rating section with its embedded Brief Psychiatric Rating Scale (Overall \& Gorham, 1962) were used to derive measures of the psychosis dimensions. In order to calculate measures of frequency of psychopathological experiences, such as lifetime cumulative incidence and interval incidence rates, as well as persistence estimates, discrete variables indicating their presence or absence across interview waves were, per definition, necessary.

Reality distortion, expressed as psychotic experiences, was the focus of the current analyses. All expressions of psychotic experiences, regardless of the presence of a formal psychotic disorder, were included. Psychotic experiences were assessed at T2 and T3 using the DIA-X/M-CIDI core psychosis section on delusions (14 items), hallucinations (5 items) and passivity (1 item). Specifically, items used were G1, G2a, G3-5, G7-G13, G13b, G14, G17, G18, G20, G20c, G21 and G22a. These items concern classic psychotic symptoms including but not limited to persecution, thought interference and passivity phenomena. Participants were first asked to read a list of all the psychotic experiences and then asked whether they ever experienced such symptoms by the psychologist (list and phrasing available upon request). All of these psychosis items can be rated in two 
ways: 1 (absent) and 5 (present), without intermediate levels. The presence of positive psychotic experiences was defined as any rating of " 5 " on any of the 20 DIA-X/M-CIDI core psychosis items (Dominguez et al., 2009). In order to examine dose-response as a function of level of psychotic experiences, an additional variable was constructed reflecting the presence of $0,1,2$ or 3 or more psychotic experiences (hereafter: Psychosis Load).

\section{Assessment of Psychotic Impairment}

In order to assess functional impact of psychotic experiences, secondary dysfunction and help-seeking behavior were examined in individuals with evidence of DIA-X/M-CIDI psychotic experiences at T2 and T3; the following procedures were followed as described in an earlier report (Dominguez et al., 2009). First, two DIA-X/M-CIDI psychosis section items for help-seeking were used: G16 (delusions) and G23 (hallucinations). These items were phrased as follows: "Did you tell a doctor about ... (insert the psychosis section beliefs/experiences previously acknowledged by the participant along with a visual representation from the response booklet) you have had?" These items were rated in a dichotomous manner $(0=$ no, $1=$ yes $)$. In addition, participants were shown a list on which several types of outpatient or inpatient institutions for mental health problems were mentioned, ranging from general practitioner or school psychologist to psychiatric sheltered housing, and asked whether they had ever sought help at any of these institutions because of psychotic symptoms as elicited in the DIA-X/M-CIDI Gsection. This item was rated in a dichotomous manner $(0=$ no, $1=$ yes $)$. Using these three help-seeking items, a dichotomous variable "Help-seeking" was constructed, indicating whether help-seeking behavior had been present (1) or absent (0).

Second, the level of dysfunction related to psychotic experiences was assessed using the DIA-X/M-CIDI items G28, G29, G29a and G36. The dysfunction score assessed the effect of the psychotic experiences on: (i) feeling upset, unable to work, go places, or enjoy oneself, at the time of having these experiences (item G28); (ii) being less able to work since these experiences began (item G29); (iii) being less able to make friends or enjoy social relationships since these experiences began (item G29a); (iv) how much their life and everyday activities were impaired when these experiences were at their worst (item G36). These four psychosis section items were rated in a dichotomous manner $(0=$ no, $1=$ yes $)$. A dichotomous variable 'Dysfunction' was constructed, representing a positive answer on any of the four questions (value label 1) versus negative answers on all four questions (value label 0).

Based on these two assessments, a combined outcome was created (hereafter 'Psychotic impairment'). Psychotic impairment was absent and scored as ' 0 ' for 
subjects scoring ' 0 ' on both help seeking behavior and dysfunction. Subjects scoring ' 1 ' on either or both help seeking behavior and dysfunction scored ' 1 ' on Psychotic Impairment.

Validation of this variable using third variables was presented previously (Dominguez et al., 2009), using two variables: Caseness and Antipsychotic Treatment. Briefly, The X16 DIA-X/M-CIDI item rated the interviewer's opinion regarding clinical evidence of psychological ill-health in 4 levels: essentially not noticeable (0), not very noticeable (1), clearly ill (2), and very ill (3). The dichotomous variable 'Caseness' indicated individuals with a noticeable level of psychiatric caseness (any score above "1"). As part of the CIDI treatment module, participants were shown a list of different types of medication, rating their use because of any psychopathological or psychosomatic problem. The acknowledgement of any antipsychotic medication (Q1EA4) reported at T2 and T3 was used to derive treatment ('Antipsychotic Treatment': $0=$ no, $1=$ yes). Validation analyses revealed that Psychotic Impairment was strongly associated with both the Caseness $(\mathrm{OR}=10.3,95 \% \mathrm{CI}=7.0-15.2)$ and the Antipsychotic Treatment $(\mathrm{OR}=15.3$, $95 \% \mathrm{CI}=6.1-38.4)$ variable.

\section{Assessment of Affective Dysregulation}

Affective dysregulation was assessed at T2 and T3 using the 28 symptom items (DSM-IV and ICD-10) of the DIA-X/M-CIDI depression and dysthymia section (items regarding feeling depressed, loss of interest, loss of energy, hopelessness, decreased concentration, loss of appetite, weight loss, sleep disturbances, feelings of worthlessness or guilt, decreased self-esteem and suicidal ideation/attempt) and the 11 symptom items of the DIA-X/M-CIDI mania section (items regarding increase in goal directed-activity, psychomotor agitation, spending sprees, sexual indiscretions, increased talkativeness, flight of ideas, increased self-esteem or grandiosity, decreased need for sleep and distractibility). Symptom items were rated either yes or no. Depression symptoms were only rated if present for at least two weeks; (hypo)mania symptoms if present for at least 4 successive days. In case the participant endorsed the presence of a particular symptom, additional probes ascertained whether the symptom was the direct result of alcohol or drug use or of physical diseases or conditions. If this were the case, the CIDI codes for substance use or somatically induced symptoms were used, and the item was not counted towards the diagnosis of a primary mood disorder. Furthermore, symptoms were only assessed and rated if at least one of the DIA-X/M-CIDI core depressive (depressed mood or loss of interest / pleasure) or core (hypo)mania symptoms (unusual happiness or excitement or unusual irritability) was present. Only participants having core (hypo)mania symptoms that were either noticed by others or because of which participants experienced problems were included. 


\section{Binary and Continuous Affective Variables}

Both binary and continuous affective variables were constructed as described previously (Krabbendam et al., 2004; Regeer et al., 2006) (Tijssen, van Os, \& Wittchen, 2009): 'Binary (hypo)mania' and 'binary depression': An a priori binary (hypo)mania variable was defined as at least two DIA-X/M-CIDI mania symptoms and a binary depression variable as at least three DIA-X/M-CIDI depression symptoms.

In addition, guided by previous work (Krabbendam et al., 2004; Regeer et al., 2006; Tijssen et al., 2009) a continuous '(hypo)mania score' and a continuous 'depression score' variable were constructed. These variables represented two continuous sum scores of symptom ratings: a sum score of (hypo)mania symptoms with a minimum of 0 , and a maximum score of 11 endorsements (hereafter: "(Hypo)mania Score"), and a sum score of depression symptoms with a minimum of 0 , and a maximum score of 28 endorsements (hereafter: "Depression Score"). Subsequent, we created, for both symptom groups, progressively stricter and overlapping subcategories of these sum scores, indicating the degree of symptom loading. For depression, 6 categories were created of no symptoms (0), at least 1 symptom (1), at least 3 symptoms (2) at least 5 symptoms (3), at least 7 symptoms (4) and at least 9 symptoms (5). For (hypo)mania, the categories were similar except that the highest category was at least 5 symptoms due to the very small number of subjects with 6 or more symptoms.

\section{Statistical Analysis}

Analyses were conducted in STATA, version 10 (STATACORP, 2008). Associations were expressed as odds ratios (OR) and 95\% confidence intervals $(95 \% \mathrm{CI})$ derived from logistic regression.

\section{Associations between Affective Dysregulation and Psychotic Experiences}

Cumulative incidence measures of affective dysregulation and psychotic experiences up until $\mathrm{T} 2$ were tested for association in order to establish their lifetime comorbidity (risk set $\mathrm{n}=2524$ ). Similarly, interval incidence of psychotic experiences at T3 (i.e. individuals with psychotic experiences at T3 free from psychotic experiences at T2) were modeled as a function of measures of level of affective dysregulation at $\mathrm{T} 3$ (risk set $\mathrm{n}=1564$ ). This latter analysis provided a temporally more precise test of the hypothesis that affective dysregulation accompanies the onset of psychotic experiences. Finally, T2 and T3 associations between individual psychotic experiences on the one hand and the level of depressive and (hypo)mania symptoms on the other were tested in order to gain insight in possible patterns of association between specific psychotic experiences and affective dysregulation. 


\section{Psychosis Persistence}

In order to assess the association between level of affective dysregulation and persistence of psychotic experiences over the period T2-T3, associations between T3 level of affective dysregulation and T3 psychotic experiences were assessed in individuals with evidence for psychotic experiences at T2 (risk set $n=464$ ).

\section{Psychotic Impairment}

In order to assess the relationship between level of affective dysregulation and incident Psychotic Impairment, associations between T3 Psychotic Impairment and T3 level of affective dysregulation were assessed in individuals with evidence of psychotic experiences at T3 but free from Psychotic Impairment at T2 (risk set $\mathrm{n}=191)$.

\section{Results}

At T2, over half (51\%) of the sample was male and the mean age was 21.7 years (SD 3.4). At T2, 574 (23\%) of all subjects presented with one or more lifetime psychotic experiences, with a stronger representation of delusions (21\%) compared to hallucinations $(5 \%)$. At $\mathrm{T} 3$, the rates were lower, representing only the occurrence of psychotic experiences over the interval from T2 to T3 (Table 1). At T2, 978 subjects (32\%) had experienced two or more (hypo)mania or three or more depression symptom; at T3, this was the case for 925 subjects $(31 \%)$. Within the subgroup of subjects with two or more (hypo)mania or three or more depression symptoms at T2 $(\mathrm{n}=978), 57 \%$ did not report similarly defined (hypo)mania or depression symptoms at T3. Conversely, within the subgroup of subjects that did not experience two more (hypo)mania or three or more depression symptoms at T2, $25 \%$ did present similarly defined (hypo)mania or depression symptoms at T3. The lifetime cumulative incidence up until T2 and the T2-T3 interval rates, assessed at T3, of hallucinations, delusions, (hypo)mania and depression symptoms are provided in Table 1.

\section{Affective Dysregulation and Reality Distortion Co-Occurrence}

Of the 574 subjects with psychotic experiences, 35\% also presented with at least 2 (hypo)mania symptoms at T2, compared to $15 \%$ of subjects without lifetime psychotic experiences at T2. At T3, these figures were $27 \%$ and $11 \%$, respectively. Similarly, $43 \%$ of the 574 subjects with psychotic experiences presented with at least 3 depression symptoms at T2, compared to $23 \%$ of subjects without psychotic experiences. At T3, these figures were $46 \%$ and $31 \%$, respectively. At both T2 and T3, the majority of psychotic experiences occurred in the context of affective dysregulation (at least 2 (hypo)mania symptoms or at least 3 depression symptoms; Table 2). 
Table 1. Lifetime Cumulative Incidence (T2) and Interval Rates (T3) of various Symptoms.

\begin{tabular}{llll} 
& T2 (n, total n; \%) & T3 (n, total n; \%) & T3 (n, total n; \%) \\
\hline $\begin{array}{l}\text { Ever experienced hallucinations } \\
\text { (at least 1) }\end{array}$ & $117(2524 ; 4.6 \%)$ & $69(2210 ; 3.1 \%)$ & $51(1937 ; 2.6 \%)$ \\
$\begin{array}{l}\text { Ever experienced delusions } \\
\text { (at least 1) }\end{array}$ & $529(2524 ; 21.0 \%)$ & $248(2210 ; 11.2 \%)$ & $121(1598 ; 7.6 \%)$ \\
$\begin{array}{l}\text { Ever experienced hallucinations or } \\
\text { delusions } \\
\text { (at least 1 symptom) }\end{array}$ & $574(2524 ; 22.7 \%)$ & $274(2210 ; 12.4 \%)$ & $128(1564 ; 8.2 \%)$ \\
$\begin{array}{l}\text { (Hypo)mania symptoms } \\
\text { (at least 2 symptoms) }\end{array}$ & $495(2525 ; 19.6 \%)$ & $285(2210 ; 12.9 \%)$ & $121(1598 ; 7.6 \%)$ \\
$\begin{array}{l}\text { Depressive symptoms } \\
\text { (at least } 3 \text { symptoms) }\end{array}$ & $695(2529 ; 27.5 \%)$ & $780(2210 ; 35.3 \%)$ & $392(1391 ; 28.2 \%)$ \\
\hline
\end{tabular}

Note. For each clinical characteristic, the size of the sample varies due to missing data on individual variables. ${ }^{a}$ Analyses were performed in the complete sample at T3, regardless of presence of psychotic experiences at T2. ${ }^{\mathrm{b}}$ Analyses were performed in the T3 sample free of the specific type of symptom at T2, thus representing interval incidence of symptoms (e.g. the interval incidence of (hypo)mania symptoms at T3 in subjects without (hypo)mania symptoms at T2 was $7.6 \%$ ).

Table 2. Frequency of Affective Dysregulation as a Function of Presence of Psychotic Experiences at T2 and T3

\begin{tabular}{|c|c|c|c|c|}
\hline & & $\begin{array}{l}\text { At least } 2 \\
\text { (hypo)mania } \\
\text { symptoms present }\end{array}$ & $\begin{array}{l}\text { At least } 3 \\
\text { depression } \\
\text { symptoms } \\
\text { present }\end{array}$ & $\begin{array}{l}\text { At least } 2 \\
\text { (hypo)mania } \\
\text { symptoms and/or } \\
\text { at least } 3 \\
\text { depression } \\
\text { symptoms present }\end{array}$ \\
\hline \multirow[t]{2}{*}{$\mathrm{T} 2$} & $\begin{array}{l}\text { Psychotic experience absent } \\
(\mathrm{n}, \mathrm{n} \text { total; \%) }\end{array}$ & $295(1950 ; 15.1 \%)$ & $446(1950 ; 22.8 \%)$ & $630(1950 ; 32.3 \%)$ \\
\hline & $\begin{array}{l}\text { Psychotic experience present } \\
(\mathrm{n}, \mathrm{n} \text { total; \%) }\end{array}$ & $200(574 ; 34.8 \%)$ & $247(574 ; 43.0 \%)$ & $346(574 ; 60.3 \%)$ \\
\hline \multirow[t]{2}{*}{$\mathrm{T} 3^{\mathrm{a}}$} & $\begin{array}{l}\text { Psychotic experience absent } \\
(\mathrm{n}, \mathrm{n} \text { total; \%) }\end{array}$ & $212(1936 ; 11.0 \%)$ & $636(1936 ; 32.9 \%)$ & $756(1936 ; 39.1 \%)$ \\
\hline & $\begin{array}{l}\text { Psychotic experience present } \\
(\mathrm{n}, \mathrm{n} \text { total; \%) }\end{array}$ & $73(274 ; 26.6 \%)$ & $144(274 ; 52.6 \%)$ & $169(274 ; 61.7 \%)$ \\
\hline \multirow[t]{2}{*}{$\mathrm{T} 3^{\mathrm{b}}$} & $\begin{array}{l}\text { Psychotic experience absent } \\
(\mathrm{n}, \mathrm{n} \text { total; \%) }\end{array}$ & $147(1436 ; 10.2 \%)$ & $420(1436 ; 29.3 \%)$ & $506(1436 ; 35.2 \%)$ \\
\hline & $\begin{array}{l}\text { Psychotic experience present } \\
(\mathrm{n}, \mathrm{n} \text { total; \%) }\end{array}$ & $33(128 ; 25.8 \%)$ & $60(128 ; 46.9 \%)$ & $70(128 ; 54.7 \%)$ \\
\hline
\end{tabular}

Note. For each clinical characteristic, the size of the sample varies due to missing data on individual variables.

${ }^{\mathrm{a}}$ Analyses were performed in the complete sample, regardless of presence of psychotic experiences at T2.

${ }^{b}$ Analyses were performed in the sample free of the specific type of symptom at T2. For example, first column, last row: the proportion of people with at least 2 (hypo)mania symptoms present in those with psychotic experiences at T3 and no hypomania symptoms at T2 was $25.8 \%$. 


\section{Bi-directional Dose-response Associations between Affective Symptoms and Reality Distortion}

The probability of lifetime psychotic experiences at $\mathrm{T} 2$ was progressively higher with greater level of co-occurrent affective dysregulation in a dose-response fashion ((Hypo)mania Score OR linear trend over 4 levels=1.70, 95\%CI: 1.55, 1.87; $\mathrm{p}<.001$; Depression Score OR linear trend over 6 levels: OR=1.31, 95\%CI: 1.24, $1.38 ; \mathrm{p}<.001$; Table 3). Incident psychotic experiences at T3 (i.e. psychotic experiences at $\mathrm{T} 3$ in subjects free from psychotic experiences at $\mathrm{T} 2$ ) were similarly associated with (Hypo)mania Score and Depression Score in a dose-response fashion (Table 3).

Similarly, the above OR linear trends expressing the association between psychotic experiences and affective dysregulation became progressively greater at higher level of Psychosis Load, both for mania and depression, and both at T2 and at T3 (Table 4).

\section{Psychosis Persistence}

(Hypo)mania Score and Depression Score were associated with persistence of psychotic experiences over the period T2-T3. Thus, within the sample of subjects with psychotic experiences at $\mathrm{T} 2$ and interviewed again at $\mathrm{T} 3$ (risk set $\mathrm{n}=464$ ), the probability of persistence of psychotic experiences was progressively greater with higher (Hypo)mania Score (OR linear trend 4 categories $=1.51$; 95\%CI: 1.22, 1.88; $\mathrm{p}<0.001$ ) and higher Depression Score (OR linear trend 6 categories=1.15; $95 \% \mathrm{CI}=1.03,1.28 ; \mathrm{p}=0.012)$.

\section{Psychotic Impairment}

Two-hundred and twenty-eight subjects (9\%) presented at T2 with Psychotic Impairment. At T3, the number with Psychotic Impairment was 118 (5\%). Of these 118, 70 subjects had not presented previously with Psychotic Impairment at T2, representing incident Psychotic Impairment. Affective dysregulation was associated with incident Psychotic Impairment: within the sample of subjects without Psychotic Impairment at T2 and presence of at least one psychotic experience at T3 (risk set $\mathrm{n}=191$ ), the probability of Psychotic Impairment at T3 was progressively higher with higher (Hypo)mania Score at T3 (OR linear trend 4 categories=1.37, 95\%CI: 1.02, 1.83; $\mathrm{p}=.036$ ) and higher Depression Score at T3 (OR linear trend 6 categories $=1.28,95 \% \mathrm{CI}: 1.10,1.49 ; \mathrm{p}=.002)$. 


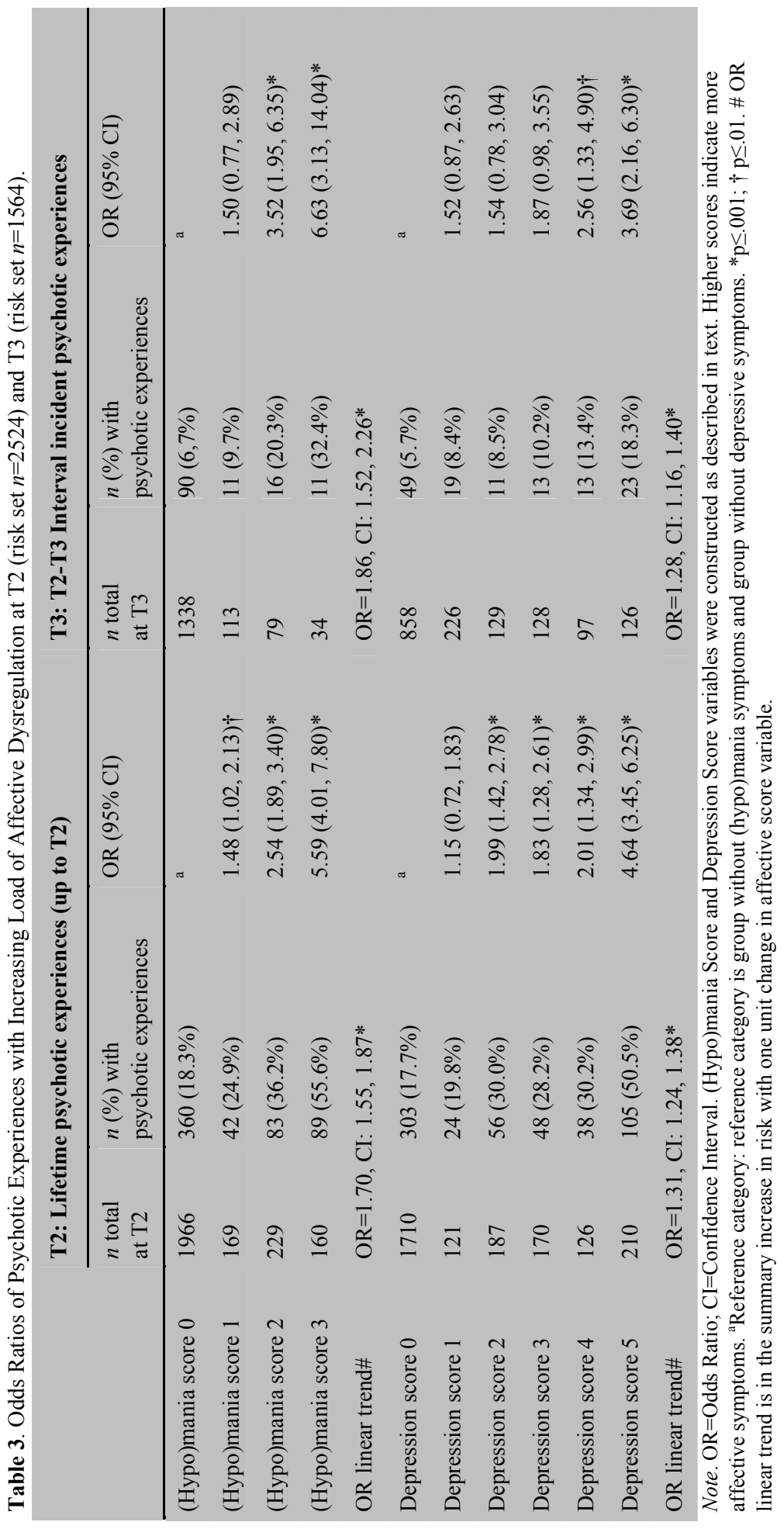




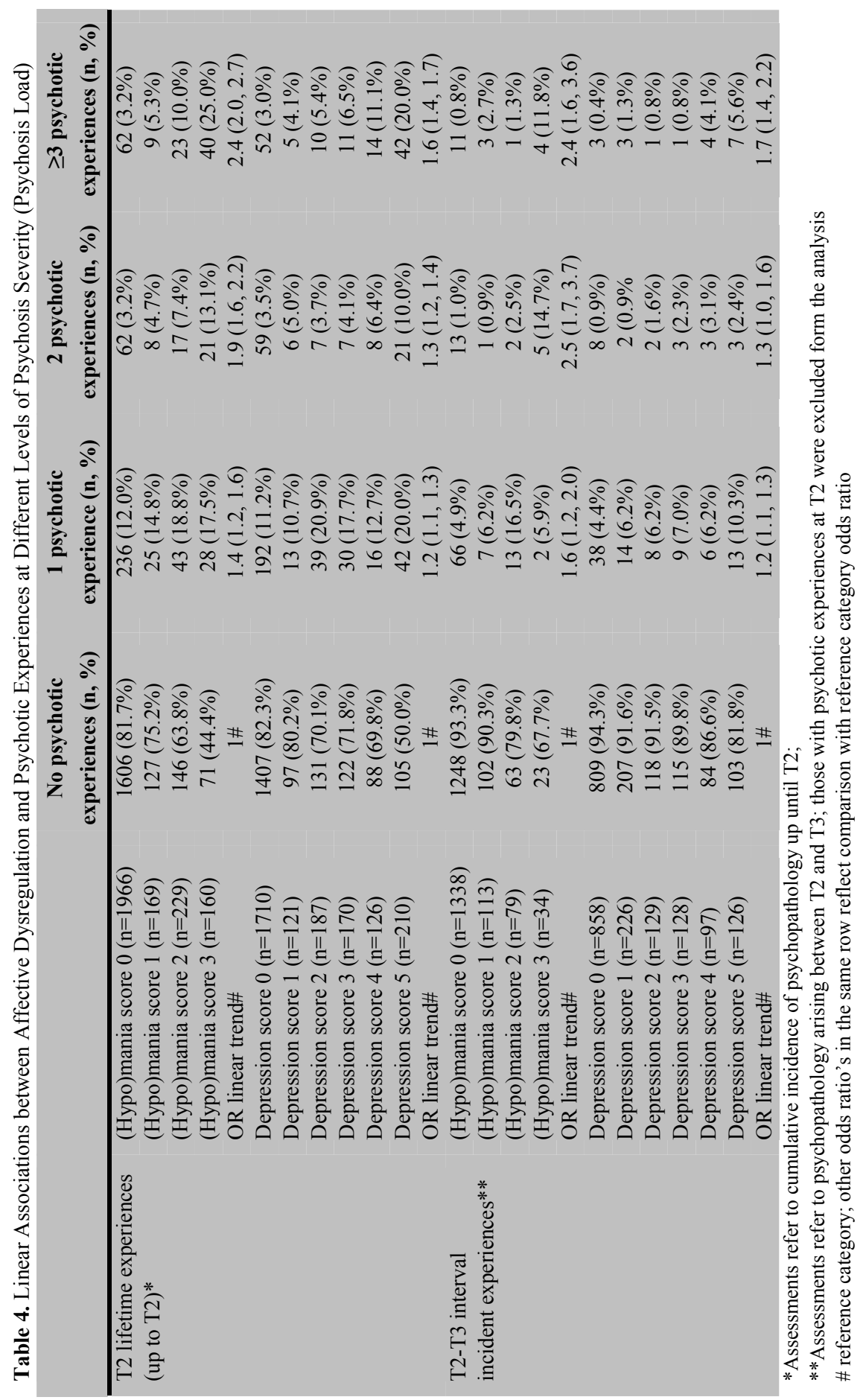




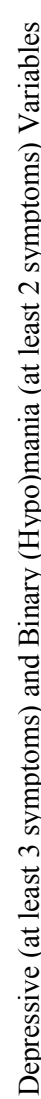

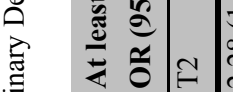

(1)

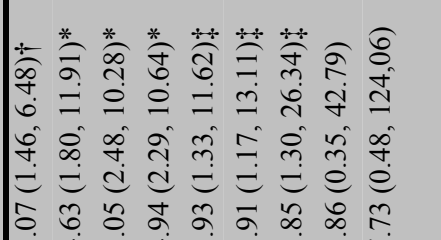

$8 \stackrel{+}{\stackrel{5}{\rightarrow}}$

竎

ते กิ

巳.

m.

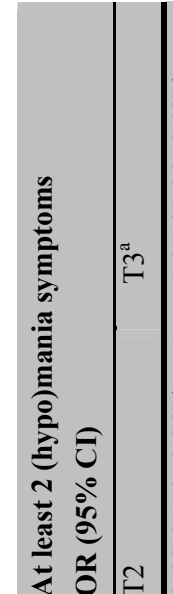

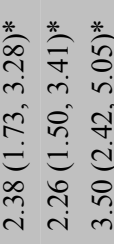

$\overbrace{0}^{*} \stackrel{*}{\infty}$

* $\frac{*}{\hat{\sigma}} \stackrel{*}{*} \frac{*}{6}+$

+.

t)

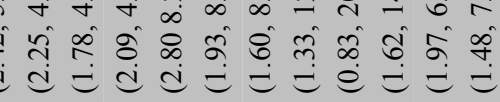

$\underset{0}{\cos } \underset{\infty}{2}=$

ง

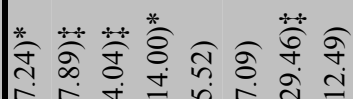

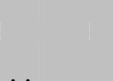

+4.

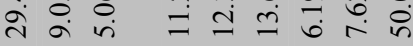

ำ

¿e é $\dot{e}=\dot{e} e$

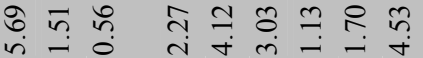

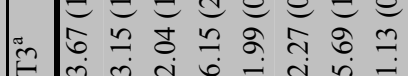

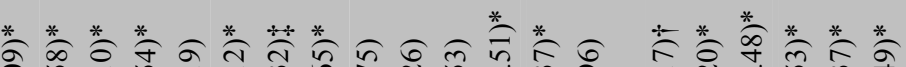

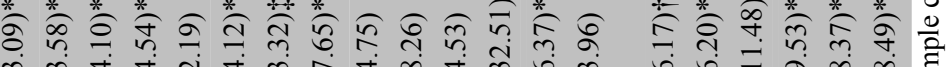



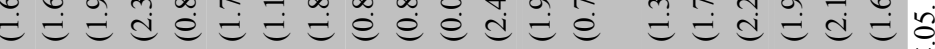

ขै ๆ

焉

气

๓ สิ สิ



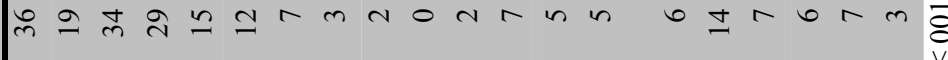

ठ‥

f 응

娄 II

s:

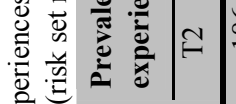

齐品

?.

芯志

에

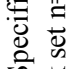

की

들

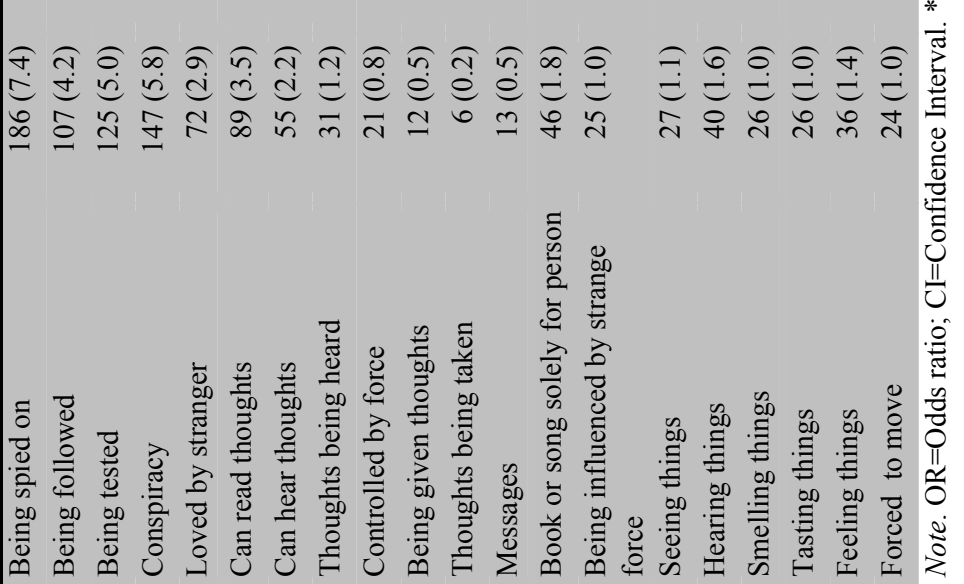




\section{Associations between Affective Dysregulation and Specific Psychotic Experiences}

An overview of specific psychotic experiences and associations with binary depression (at least three symptoms) and (hypo)mania (at least two symptoms) variables is provided in Table 5. For both depression and (hypo)mania symptoms, no specific trend or pattern was apparent among the different psychotic experiences.

\section{Discussion}

Clinical studies in help-seeking samples suggest that early intervention in the prodromal phase of psychotic disorder may be feasible (Cannon, Cadenhead, Cornblatt, \& 2008). However, knowledge on the developmental mechanisms underlying the epidemiology of prodromal states in the general population remains very limited. The current study connects reality distortion and affective dysregulation in a general population setting from a perspective of risk and a perspective of understanding links with normal mentation. The results suggest that psychotic experiences in the general population are non-specifically associated with affective dysregulation, in a bi-directional dose-response fashion. Second, persistence of psychotic experiences and the onset of impairment associated with reality distortion occur more often if there is a context of affective dysregulation.

\section{Co-expression as a Reflection of Overlapping Genetic Liabilities}

Although the delusions and hallucinations of psychosis can be readily recognized, classifying psychotic states remains a major challenge. Psychosis is not exclusive to schizophrenia and occurs across a range of diagnostic categories of psychotic disorder, and even amongst the category of non-psychotic mood disorders (Hanssen et al., 2003). The criteria used to distinguish between the different categories of psychotic disorder are based on duration, dysfunction, associated substance use, "bizarreness" of delusions and presence of depression or (hypo)mania. However, the resulting diagnostic categories of psychotic disorder show overlap in genetic liability amongst themselves. For example, Kendler and colleagues (Kendler et al., 1993) demonstrated a significant familial relationship between non-schizophrenic psychotic disorders with both schizophrenia and schizotypal personality disorder. Family and twin studies similarly demonstrate a degree of overlap in genetic liability between non-affective psychotic disorder and bipolar disorder (Cardno et al., 2002; Craddock \& Owen, 2007; Lichtenstein et al., 2009).

Co-expression of (subclinical) affective dysregulation and psychotic experiences in general cohort studies may thus in part reflect overlapping distributed genetic vulnerabilities. The majority of general cohort studies on transition rates from subclinical symptoms to clinical syndromes did not examine 
co-expression of reality distortion and affective dysregulation. Multiple studies suggest that subclinical psychotic experiences increase the risk for non-affective psychotic disorder (Chapman et al., 1994; Dominguez et al., 2009; Hanssen et al., 2005; Poulton et al., 2000; Welham et al., 2008; Werbeloff et al., 2009) and similarly subthreshold depression and/or (hypo)mania symptoms have been shown to increase the risk for bipolar disorder (Regeer et al., 2006; Tijssen et al., 2009). Overall, affective dysregulation and reality distortion have been shown to represent the behavioral expression of risk for more severe psychotic states including schizophrenia and bipolar disorder in the general population. In light of the accumulating evidence on overlap in genetic liability (Cardno et al., 2002; Craddock \& Owen, 2007; Lichtenstein et al., 2009), suggesting a broad underlying vulnerability that expresses across the different categories, our findings of associations between affective dysregulation and psychotic experiences in the general population may therefore simply reflect passive clustering of the behavioral expression of overlapping genetic risks.

\section{Co-expression as a Reflection of Causal Influence}

The fact that co-expression of affective dysregulation predicted persistence of reality distortion and impairment associated with psychotic experiences suggest that, in addition to correlated genetic liabilities, a direct impact of affective dysregulation on the onset of psychotic experiences may be hypothesized. Existent psychological models of symptom formation suggest that the emotional context, and associated beliefs or appraisals, may induce bias in logical reasoning processes and therefore provoke reality distortion in healthy individuals (Garety et al., 2001; Myin-Germeys \& van Os, 2007). In the psychological model described by Garety and colleagues (Garety et al., 2007), the experience of a stressful event is thought to potentially give rise to altered pre-existing beliefs and ongoing appraisals of experiences, due to a certain emotional change or a cognitive processing bias. These distorted processes may lead to aberrant experiences that may seem personally significant to the individual and are likely to trigger a search for an explanation as to their meaning and cause that is consistent with affect-associated beliefs. Biased appraisal processes may contribute to a judgment that the experience is in fact externally caused. It is the interpretation that causes the associated distress and disability, rather than the experience itself. Thus, maladaptive appraisal patterns, induced by emotional processes, are suggested to increase the risk for positive symptom formation in vulnerable individuals (Garety et al., 2007). Our findings provide support for a cognitive model of symptom formation, by demonstrating involvement of affective processes in the onset and persistence of reality distortion outside the context of disorder. 


\section{Linking Genetic and Cognitive Mechanisms}

Evidence on the role of cognitive processes suggests that cognitive biases and appraisals can help explain onset of psychosis (Garety et al., 2007). Attempts have been made to integrate cognitive and neurobiological theories into a single model of psychotic states (Garety et al., 2007; Kapur, 2003; van Os, 2009). Thus, evidence indicates that genetic risk for psychotic disorder is associated with underlying alterations in the dopamine system, including increased dopamine synaptic availability (Hirvonen, van Erp, \& Huttunen, 2005), increased striatal dopamine synthesis (Huttunen, Heinimaa, \& Svirskis, 2008; Meyer-Lindenberg, Miletich, \& Kohn, 2002) and increased dopamine reactivity to stress (Brunelin et al., 2008; Myin-Germeys, Marcelis, Krabbendam, Delespaul, \& van Os, 2005). Under normal circumstances, it is the context-driven activity of the dopamine system that mediates the experience of novelty and the acquisition of appropriate motivational salience, detecting new rewards in the environment that facilitate learning and goaldirected behavior. Certain cerebral vulnerabilities, occasioned by interplay between genetic and environmental risks, could trigger context-independent or contextinappropriate release of dopamine. A dysregulated dopamine system may cause aberrant assignment of salience and motivational significance to external objects (leading to delusions) and internal representations of percepts and memories (leading to hallucinations) (Kapur, Mizrahi, \& Li, 2005).Thus, theory derived from existent psychological models of psychotic symptom formation predicts that affective dysregulation may impact directly on risk for reality distortion. An integrated model would additionally suggest that the risk to develop a clinical disorder is particularly high in those who additionally have a genetic liability for dopaminergic dysregulation, facilitating aberrant salience attribution.

\section{Limitations}

Several limitations should be taken into account when interpreting these results. First, the study was epidemiological, and no direct measurements of affective and cognitive processes such as aberrant salience attribution were available, limiting the explanatory power of the findings on these mechanisms. Second, although longitudinal, measurements were too far apart for dynamic models of the onset of impairment as a function of affective dysregulation. Third, assessment of psychotic experiences, while better than lay-interviewer assessed self-reports, will likely contain false positive answers even when interviewers are clinical psychologists. However, it is unlikely that false positive assessments would produce spurious associations with affective dysregulation - the opposite, more conservative alternative is more likely. Furthermore, the substantial literature on self-reported psychotic experiences, including those assessed with the DIA-X/M-CIDI, indicates substantial predictive and other forms of validity of these phenomena (van Os et al., 2009). Fourth, lifetime rates in excess of $22 \%$ may seem high, given an estimate of 
5\%-8\% in a recent systematic review (van Os et al., 2009). However, in another systematic review we are preparing, it is apparent that rates of psychotic experiences are critically dependent on the number of items assessing different psychotic experiences. Previous work using the CIDI also detected rates close to $20 \%$ (van Os, Hanssen, Bijl, \& Ravelli, 2000). Finally, it could be argued that the measure of psychotic impairment used was broad, resulting in a lifetime rate of $9 \%$, which may be considered very high for psychosis. The high rate is in part inherent to the population-based research paradigm of EDSP, which will always detect many more cases compared to the much lower administrative rates reflecting treatment at the level of services. Furthermore, even the rate of narrowly defined clinical psychotic disorder, when assessed completely, may be as high as 3.5\% (Perala et al., 2007). In addition, to the degree that our definition of impairment was broad, it can be argued that in this context, sensitivity is more important than specificity, given the fact that the main clinical application of research on extended phenotypes ultimately is situated in the area of early detection.

\section{Funding}

This work is part of the Early Developmental Stages of Psychopathology (EDSP) Study. The EDSP-Study is funded by the German Federal Ministry of Education and Research [BMBF; project no. 01EB9405/6, 01EB 9901/6, EB01016200, 01EB0140, and 01EB0440]. Part of the field work and analyses were also additionally supported by grants of the Deutsche Forschungsgemeinschaft [DFG; project no. LA1148/1-1, WI2246/1-1, WI 709/7-1, and WI 709/8-1].

\section{Acknowledgements}

Data Access and Responsibility: Principal investigators of the EDSP-Study are Dr. Hans-Ulrich Wittchen and Dr. Roselind Lieb. Both take responsibility for the integrity of the study data. All authors and co-authors had full access to all study data. Data analysis and manuscript preparation were completed by the authors and co-authors of this article, who take responsibility for its accuracy and content.

Core staff members of the EDSP group are: Dr Katja Beesdo, Dr. Petra Zimmermann, Dr. Axel Perkonigg, PhD Dipl.-Stat. Michael Höfler, , Dipl.-Psych. Tanja Brückl, Dipl.-Psych. Agnes Nocon, Dipl.-Inf. Hildegard Pfister, Dipl.-Soz. Barbara Spiegel, Dipl.-Psych. Andrea Schreier. Scientific advisors are Dr. Jules Angst (Zurich), Dr. Kathleen Merikangas (NIMH, Bethesda), Dr. Ron Kessler (Harvard, Boston) and Dr. Jim van Os (Maastricht).The EDSP project and its family genetic supplement has been approved by the Ethics Committee of the Faculty of the Technische Universität Dresden (No: EK-13811). All participants provided informed consent. 
Financial Disclosure: I. van Rossum: also employee of Eli Lilly; M.D.G. Dominguez: Nothing to report; Dr. Lieb: Speaker Honoraria: Wyeth; Dr. Wittchen: Research Support: Novartis, Pfizer; Schering-Plough. Consulting: Novartis, Pfizer, Wyeth, Organon, Lundbek. Speaker Honoraria: Novartis, Schering-Plough, Pfizer, Wyeth, Servier.; Dr. van Os is/has been an unrestricted research grant holder with, or has received financial compensation as an independent symposium speaker from Eli Lilly, BMS, Lundbeck, Organon, Janssen-Cilag, GSK, AstraZeneca, Pfizer and Servier - companies that have an interest in the treatment of psychosis.

\section{References}

Bentall, R., Rowse, G., \& Shryane, N. (2009). The cognitive and affective structure of paranoid delusions: a transdiagnostic investigation of patients with schizophrenia spectrum disorders and depression. Archives of General Psychiatry, 66(3), 236-247.

Brunelin, J., d'Amato, T., van Os, J., Cochet, A., Suaud-Chagny, M., \& Saoud, M. (2008). Effects of acute metabolic stress on the dopaminergic and pituitary-adrenal axis activity in patients with schizophrenia, their unaffected siblings and controls. Schizophrenia Research, 100(1-3), 206-211.

Cannon, T., Cadenhead, K., Cornblatt, B., \& (2008). Prediction of psychosis in youth at high clinical risk: a multisite longitudinal study in North America. Archives of General Psychiatry, 65(1), 28-37.

Cannon, T., Cornblatt, B., \& McGorry, P. (2007). The empirical status of the ultra high-risk (prodromal) research paradigm. Schizophrenia Bulletin, 33(3), 661-664.

Cardno, A., Rijsdijk, F., Sham, P., Murray, R., \& McGuffin, P. (2002). A twin study of genetic relationships between psychotic symptoms. American Journal of Psychiatry, 159(4), 539-545.

Chapman, L., Chapman, J., Kwapil, T., Eckblad, M, \& Zinser, M. (1994). Putatively psychosis-prone subjects 10 years later. Journal of Abnormal Psychology, 103(2), 171-183.

Cougnard, A., Marcelis, M., \& Myin-Germeys, I. (2007). Does normal developmental expression of psychosis combine with environmental risk to cause persistence of psychosis? A psychosis proneness-persistence model. Psychological Medicine, 37(4), 513-527.

Craddock, N., \& Owen, M. (2007). Rethinking psychosis: the disadvantages of a dichotomous classification now outweigh the advantages. World Psychiatry, 6(2), 84-91.

Debbane, M., van der Linden, M., Gex-Fabry, M., \& Eliez, S. (2009). Cognitive and emotional associations to positive schizotypy during adolescence. Journal of Child Psychology and Psychiatry, 50(3), 326-334.

Dominguez, M., Wichers, M., Lieb, R., Wittchen, H., \& van Os, J. (2009). Evidence that onset of clinical psychosis is the outcome of progressively more persistent subclinical psychotic experiences: an 8-year cohort study. Schizophrenia Bulletin, PUBMED publication:doi:10.1093/schbul/sbp022.

Dunayevich, E., \& Keck, P. (2000). Prevalence and description of psychotic features in bipolar mania. Current Psychiatry Reports, 2(4), 286-290.

Garety, P., Bebbington, P., Fowler, D., D, F., \& Kuipers, E. (2007). Implications for neurobiological research of cognitive models of psychosis: a theoretical paper. Psychological Medicine, 37, 1377-1391.

Garety, P., Kuipers, E., Fowler, D., Freeman, D., \& Bebbington, P. (2001). A cognitive model of the positive symptoms of psychosis. Psychological Medicine, 31(2), 189-195.

Green, C., Garety, P., \& Freeman, D. (2006). Content and affect in persecutory delusions. British Journal of Clinical Psychology, 45(4), 561-577.

Hanssen, M., Bak, M., Bijl, R., Vollebergh, W., \& van Os, J. (2005). The incidence and outcome of subclinical psychotic experiences in the general population. British Journal of Clinical Psychology, 44(2), 181-191.

Hanssen, M., Peeters, F., Krabbendam, L., Radstake, S., Verdoux, H., \& van Os, J. (2003). How psychotic are individuals with non-psychotic disorders? Social Psychiatry and Psychiatric Epidemiology, 38(3), 149154.

Hirvonen, J., van Erp, T., \& Huttunen, J. (2005). Increased caudate dopamine D2 receptor availability as a genetic marker for schizophrenia. Archives of General Psychiatry, 62(4), 371-378. 
Holt, D., Tinone, D., Long, S., Goff, D., Cather, C., Raugh, S., et al. (2006). The misattribution of salience in delusional patients with schizophrenia. Schizophrenia Research, 83, 247-256.

Huttunen, J., Heinimaa, M., \& Svirskis, T. (2008). Striatal dopamine synthesis in first-degree relatives of patients with schizophrenia. Biological Psychiatry, 63(1), 114-117.

Kapur, S. (2003). Psychosis as a state of aberrant salience: a framework linking biology, phenomenology and pharmacology in schizophrenia. American Journal of Psychiatry, 160, 13-23.

Kapur, S., Mizrahi, R., \& Li, M. (2005). From dopamine to salience to psychosis: linking biology, pharmacology and phenomenology of psychosis. Schizophrenia Research, 79, 59-68.

Kaymaz, N., van Os, J., De Graaf, R., Ten Have, M., Nolen, W., \& Krabbendam, L. (2007). The impact of subclinical psychosis on the transition fro subclinical mania to bipolar disorder. Journal of Affective Disorders, 98, 55-64.

Kendler, K., McGuire, M., Gruenberg, A., Spellman, M., O'Hare, A., \& Walsh, D. (1993). The Roscommon family study II. The risk of nonschizophrenic nonaffective psychoses in relatives. Archives of General Psychiatry, 50(8), 645-652.

Krabbendam, L., Myin-Germeys, I., Bak, M., \& van Os, J. (2005). Explaining transitions over the hypothesized psychosis continuum. . Australian New-Zealand Journal of Psychiatry, 39(3), 180-196.

Krabbendam, L., Myin-Germeys, I., De Graaf, R., Vollebergh, W., Nolen, W., \& van Os, J. (2004). Dimensions of depression, mania and psychosis in the general population. Psychological Medicine, 34, 1177-1186.

Krabbendam, L., Myin-Germeys, I., Hanssen, M., De Graaf, R., Vollebergh, W., Bak, M., et al. (2005). Development of depressed mood predicts onset of psychotic disorder in individuals who report hallucinatory experiences. British Journal of Clinical Psychology, 44, 113-125.

Krabbendam, L., \& van Os, J. (2005). Affective processes in the onset and persistence of psychosis. European Archives on Psychiatry and Clinical Neuroscience, 255, 185-189.

Lichtenstein, P., Yip, B., \& Bjork, C. (2009). Common genetic determinants of schizophrenia and bipolar disorder in Swedish families: a population-based study. Lancet, 373(9659), 234-239.

Lieb, R., Isensee, B., Von Sydow, K., \& Wittchen, H. (2000). The early developmental stages of psychopathology study (EDSP): a methodological update. European Addiction Research, 6(4), 170-182.

Meyer-Lindenberg, A., Miletich, R., \& Kohn, P. (2002). Reduced prefrontal activity predicts exaggerated sriatal dopaminergic function in schizophrenia. Nature Neuroscience, 5(3), 267-271.

Myin-Germeys, I., Marcelis, M., Krabbendam, L., Delespaul, P., \& van Os, J. (2005). Subtle fluctuations in psychotic phenomena as functional states of abnormal dopamine reactivity in individuals at risk. Biological Psychiatry, 58(2), 105-110.

Myin-Germeys, I., \& van Os, J. (2007). Stress-reactivity in psychosis: evidence for an affective pathway to psychosis. Clinical Psychology Review, 27(4), 409-424.

Overall, J., \& Gorham, D. (1962). The brief psychiatric rating scale. Psycholology Reports, 10, 799-812.

Perala, J., Suvisaari, J., Saarni, S. I., Kuoppasalmi, K., Isometsa, E., Pirkola, S., et al. (2007). Lifetime prevalence of psychotic and bipolar I disorders in a general population. Arch Gen Psychiatry, 64(1), 1928.

Peralta, V., \& Cuesta, M. (2009). Characterization of affective domains within the nonaffective psychotic disorders. Schizophrenia Research, 111(1-3), 61-69.

Poulton, R., Caspi, A., Moffitt, T., Cannon, M., Murray, R., \& Harrington, H. (2000). Children's self-reported psychotic symptoms and adult schizophrenicform disorder: a 15-year longitudinal study. Archives of General Psychiatry, 57(11), 1053-1058.

Reed, V., Gander, F., Pfister, H., Steiger, A., Sontagg, H., Trenkwalder, C., et al. (1998). To what degree does the Composite International Diagnostic Interview (CIDI) correctly identify DSM-IV disorders? Testing validity issues in a clinical sample. International Journal of Methods in Psychiatry Research, 7, 619-627.

Regeer, E., Krabbendam, L., De Graaf, R., Ten Have, M., Nolen, W., \& van Os, J. (2006). A prospective study of the transition rates of subthreshold (hypo)mania and depression in the general population. Psychological Medicine, 36, 619-627.

Sareen, J., Cox, B., Goodwin, R., \& Asmundson, G. (2005). Co-occurrence of post-traumatic stress disorder with positive psychotic symptoms in a nationally representative sample. Journal of traumatic stress, 18(4), 313-322.

Smith, B., Fowler, D., \& Freeman, D. (2006). Emotion and psychosis: links between depression, self-esteem, negative schematic beliefs and delusions and hallucinations. Schizophrenia Research, 86(1-3), 181-188. 
STATACORP. (2008). Statistical software: release 10.0: TXS Stata corporation, college station TX.

Stefanis, N., Hanssen, M., Smirnis, N., Avramopoulos, D., Evdokimidis, I., Stefanis, C., et al. (2002). Evidence that three dimensions of psychosis have a distribution in the general population. Psychological Medicine, 32, 347-358.

Tijssen, J., van Os, J., \& Wittchen, H. (2009). Evidence that bipolar disorder is the poor outcome fraction of a common developmental phenotype: an 8-year cohort study in young people. Psychological Medicine, In press.

van Os, J. (2009). A salience dysregulation syndrome. British Journal of Psychiatry, 194, 101-103.

van Os, J., Hanssen, M., Bijl, R. V., \& Ravelli, A. (2000). Strauss (1969) revisited: A psychosis continuum in the general population? Schizophr Res, 45(1-2), 11-20.

van Os, J., \& Kapur, S. (2009). Schizophrenia. Lancet, 374(9690), 635-645.

van Os, J., Linscott, R., Myin-Germeys, I., Delespaul, P., \& Krabbendam, L. (2009). A systematic review and meta-analysis of the psychosis continuum: evidence for a psychosis proneness-persistence-impairment model of psychotic disorder. Psychological Medicine, 39(2), 179-195.

Welham, J., Scott, J., \& Williams, G. (2008). Emotional and behavioural antecedents of young adults who screen positive for non-affective psychosis: a 21-year birth cohort study. Psychological Medicine, 39(4), 625-634.

Werbeloff, N., Drukker, M., \& Dohrenwend, B. (2009). Self-reported psychotic symptoms in the community are associated with increased risk of later hospitalisation for non-affective psychotic disorders. Schizophrenia Bulletin, 35(supplement 1), 74.

Wittchen, H. (1994). Reliability and validity studies of the WHO-Composite International Diagnostic Interview (CIDI): a critial review. Journal of Psychiatric Research, 28(1), 57-84.

Wittchen, H., Perkonigg, A., Lachner, G., \& Nelson, C. (1998). Early developmental stages of psychopathology study (EDSP): objectives and design. European Addiction Research, 4(1-2), 18-27.

Wittchen, H., \& Pfister, H. (1997). DIA-X-Interviews: Manual fur screening-verfahren und interview; interviewheft langsschnittuntersuchung (DIA-X-Lifetime); enganzungsheft (DIA-X-Lifetime); interviewheft Querschnittsuntersuchung (DIA-X-Monatsversion); erganzungsheft (DIA-X-12 Montasversion); PC-programm zur durchfuhrung der interviews (Langsund Querschnittsuntersuchung). Ausertungsprogramm. . Zeitlinger SA, ed. Frankfurt, Germany.

Wittchen, H., Robins, L., Cottler, L., Sartorius, N., Burke, J., \& Regier, D. (1991). Cross-cultural feasability, reliability and sources of variance of the Composite International Diagnostic Interview (CIDI). The multicenter WHO/ADAMHA Fields trials. British Journal of Psychiatry, 159, 645-653.

Zisook, S., McAdams, L., Kuck, J., Harris, M., Bailey, A., Patterson, T., et al. (1999). Depression symptoms in schizophrenia. American Journal of Psychiatry, 156, 1736-1743. 


\title{
CHAPTER 5
}

\author{
ONSET OF CLINICAL PSYCHOSIS: \\ NEGATIVE/DISORGANIZED SYMPTOMS \\ INCREASING RISK FOR POSITIVE \\ SYMPTOMS INCREASING RISK FOR \\ IMPAIRMENT?A 10-YEAR STUDY
}

Maria-de-Gracia Dominguez ${ }^{1}$, Meram Can Saka², Roselind Lieb ${ }^{3,6}$, Hans-Ulrich Wittchen ${ }^{3,4}$, Jim van Os $^{1,5}$

\footnotetext{
${ }^{1}$ Department of Psychiatry and Neuropsychology, School of Mental Health and Neuroscience, Maastricht University Medical Centre, Maastricht, The Netherlands.

${ }^{2}$ Department of Psychiatry, School of Medicine, Ankara University, Dikimevi, Mamak Caddesi, Ankara, Turkey.

${ }^{3}$ Max Planck Institute of Psychiatry, Clinical Psychology and Epidemiology Unit, Munich, Germany. ${ }^{4}$ Institute of Clinical Psychology and Psychotherapy, Technical University Dresden, Dresden, Germany. ${ }^{5}$ Division of Psychological Medicine, Institute of Psychiatry, London, UK.

${ }^{6}$ Epidemiology and Health Psychology, Institute of Psychology, University of Basel, Basel, Switzerland.
} 


\begin{abstract}
Objective

Cognitive and negative symptoms associated with schizophrenia may represent alterations in brain development associated with distributed genetic risk, influencing a final common pathway of neurotransmitter dysregulation resulting in the onset of positive psychotic symptoms, particularly when combined with environmental exposures such as cannabis, developmental trauma and urbanicity. The epidemiological predictions of this model were tested.
\end{abstract}

\title{
Method
}

A prospective cohort study was conducted in a sample of originally $n=3021$ adolescents and young adults, aged 14-24 years at outset, over a period of 8.4 years, in Munich, Germany (Early Developmental Stages of Psychopathology Study). Measures of psychopathology and clinical relevance were assessed by clinical psychologists using the Composite International Diagnostic Interview.

\section{Results}

Both negative and positive psychotic symptoms were frequent (5-year cumulative prevalence rates of around 12\%) and occurred in combination more often than predicted by chance. Negative symptoms revealed a pattern of socio-demographic associations indicative of developmental impairment, whereas the positive symptoms were associated with environmental risk factors such as trauma, cannabis use and urbanicity. Negative symptoms predicted positive symptoms over time, and co-occurrence of positive and negative symptoms was predictive of clinical relevance in terms of secondary functional impairment and help-seeking behaviour.

\section{Conclusions}

Results suggest that the liabilities underlying negative features of psychotic illness are distributed at a population level and drive the ontogenesis of positive psychotic experiences after exposure to environmental risks, increasing the likelihood of impairment and need for care.

\section{Key words}

Epidemiology, Community, Positive Symptoms, Negative Symptoms, Psychotic disorders, Psychopathology, Environment, Risk Factors. 


\section{Introduction}

Delusions and hallucinations are not exclusive to schizophrenia and psychotic disorders and occur across a range of diagnostic categories. Differential diagnosis is based on duration, symptom patterns, dysfunction, substance use, "bizarreness" of delusions and presence of depression or mania. However, the resulting diagnostic categories show overlap in genetic liability amongst themselves (Craddock \& Owen, 2007; Kendler et al., 1993). Psychotic disorders consist of different symptom dimensions that, while clustering together in the same syndrome, nevertheless also display relative contrasts. Thus, the positive and the negative symptom domains show a pattern of co-clustering in the diagnostic category of schizophrenia, but there is also evidence for a relative contrast: the negative dimension is associated with neurocognitive impairment whilst the positive is not (M. D. G. Dominguez, Viechtbauer, Simons, van Os, \& Krabbendam, 2009), and positive and negative symptoms show a differential pattern of socio-demographic and environmental risk associations (Leung \& Chue, 2000; Schultz et al., 1997), alterations in cerebral activity (Schroder et al., 1996) and alterations in neurotransmission (Laruelle, Kegeles, \& Abi-Dargham, 2003). Both dimensions also follow differential longitudinal courses (Eaton, Thara, Federman, Melton, \& Liang, 1995) and display differential associations with antipsychotic response (Erhart, Marder, \& Carpenter, 2006), social functioning (Macdonald, Hayes, \& Baglioni, 2000) and prognosis (McGlashan \& Fenton, 1992).

Research in clinical samples cannot examine to what degree associations between variable clinical characteristics and other variables may arise as a result of the illness and/or the associated treatments. This is particularly relevant with regard to negative symptom confounds such as dopamine-blocking medications, extrapyramidal symptoms, depression, social exclusion due to stigma and positive psychotic symptoms causing secondary negative symptoms (Kapur, 2004; Malla et al., 2002). Similarly, clinical research cannot determine the degree to which the processes underlying negative and positive symptoms in psychotic patients may be universal, representing quantitative variations of normal human mentation (van Os \& Kapur, 2009) or determine if and how interacting processes underlying both symptoms, from a perspective of risk, may contribute to the onset of need for care.

Systematic review of general population surveys indicates that the experiences associated with psychotic categories, such as paranoid delusional thinking and auditory hallucinations, are observed, in an attenuated form, in 5-8\% of respondents that have never been diagnosed as having any mental disorder with psychotic features (van Os, Linscott, Myin-Germeys, Delespaul, \& Krabbendam, 2009). These attenuated expressions may be conceived as the behavioural expression of underlying distributed liability for psychosis (Lataster, Myin-Germeys, Derom, 
Thiery, \& van Os, 2009). This interpretation is validated by longitudinal research showing a link between psychotic experiences in the general population and increased probability of having a psychotic disorder later in life. There is evidence from two birth cohorts (Poulton et al., 2000; Welham et al., 2009), three representative population cohorts (M. D. Dominguez, Wichers, Lieb, Wittchen, \& van Os, 2009; Hanssen, Bak, Bijl, Vollebergh, \& van Os, 2005; Werbeloff, Drukker, \& Dohrenwend, 2009) and the seminal study by Chapman (Chapman, Chapman, Kwapil, Eckblad, \& Zinser, 1994) that low grade psychotic experiences may precede the diagnosis of psychotic disorder, including hospital admission, by many years.

The above population-based work has focused on experiences resembling positive psychotic symptoms and it is not known if and how attenuated negative symptoms might play a role. It has been suggested that experiences resembling negative symptoms are also expressed in attenuated form in otherwise "healthy" individuals, and that the combination of attenuated positive and negative symptoms are "toxic" in terms of increasing the risk for need for care (Maric et al., 2004). Therefore, epidemiological research on such psychopathological phenomena may be productive, particularly when a longitudinal perspective allows for the clarification of the direction of effects and the relationship with illness onset.

In the current paper, a longitudinal, 10-year study in young people is presented. Hypotheses were derived from the suggestion that cognitive/negative symptoms associated with schizophrenia may represent alterations in brain development associated with distributed genetic risk, influencing a hypothesized final common pathway of neurotransmitter dysregulation resulting in the onset of positive psychotic symptoms, particularly when combined with environmental exposures (van Os \& Kapur, 2009). We therefore hypothesized that: (i) subclinical expressions of positive and negative symptoms are distributed in the general population and occur more often together than predicted by chance, (ii) positive symptoms are associated with environmental risks and negative features with indices of developmental impairment, (iii) negative symptoms predict psychotic experiences over time but not the other way round and, finally, (iv) "co-morbid" expression of positive and negative experiences are associated with impairment and risk for patient status.

\section{Method}

\section{Sample and Study Design}

The Early Developmental Stages of Psychopathology (EDSP) study collected data on the prevalence, incidence, risk factors and course of mental disorders in a 
representative random population sample of 14-24-years-old adolescents and young adults in Munich, Germany.

This longitudinal prospective design consisted of a baseline and 3 follow-up surveys, covering a time period of on average 1.6 years (T0-T1), 3.5 years (T0-T2) and 8.6 years (T0-T3; range $=7.3-10.5$ ). Because the primary goal of the study was to examine the incidence of and the developmental risk factors for psychopathology, the younger group (14- to 15-years) was sampled at twice the rate of persons aged 16-21 years, and the oldest group (22-24 years) was sampled at half this rate. Subjects aged 14-17 years at baseline were examined at all time-points whereas the entire baseline sample was assessed three times (T0, T2 and T3). Information on the sampling, representativeness, instruments, procedures and statistical methods of the EDSP study was previously described (Lieb, Isensee, von Sydow, \& Wittchen, 2000).

The present study is based on the 3,021 individuals aged 14-24 years at baseline and their T2 $(n=2,548$; response rate $=84 \%)$ and T3 follow-ups $(n=2,210 ; 73 \%)$. Written informed consent was obtained from all participants.

\section{Instruments}

Participants were interviewed with the Munich-Composite International Diagnostic Interview (DIA-X/M-CIDI) (Wittchen \& Pfister, 1997), an updated version of the World Health Organization's CIDI (WHO, 1990). The CIDI is a comprehensive, fully standardized computer-assisted diagnostic interview for the assessment of symptoms, syndromes, and diagnoses of various mental disorders in accordance with the DSM-IV criteria. Acceptable to high validity (Reed, Gander, \& Pfister, 1998), interrater (Wittchen et al., 1991) and test-retest reliability (Wittchen, 1994) has been established. Trained and experienced clinical psychologists conducted the interviews at baseline (lifetime version) and follow-up assessments (interval version: covering the assessment period from the last interview until the next).

\section{Assessment of Symptom Clusters}

Information from the CIDI psychosis section and the clinical interview rating section with its embedded Brief Psychiatric Rating scale (BPRS; (Overall \& Gorham, 1962)) were used to derive measures of psychopathological clusters. In order to calculate measures of frequency (prevalence, incidence and persistence estimates), discrete variables indicating presence or absence of clusters across interview waves were, per definition, necessary. 


\section{The Negative-Disorganized Cluster}

The 'Negative-Disorganized Cluster' (NDC) was designed in accordance with psychopathological findings in the early course of psychosis, showing that the "Bleulerian blend" of negative symptoms, catatonic/motor and disorganization symptoms loaded on a single factor while positive symptoms loaded on another (McGorry, Bell, Dudgeon, \& Jackson, 1998). At T0 and T3, two items concerning classic negative and disorganized symptoms from the DIA-X/M-CIDI interview ratings X-section were used: (i) indifference (X11), and (ii) thought incoherence and/or illogicality (X12). These 2 items were rated as: absent (1), very little (2), a little (3), moderately (4), moderately strong (5), a lot (6), very much (7). Each item was dichotomized: absent (0) versus present (1) indicating any score above " 1 ". For the purpose of the analyses, the T0- and T3-NDC were defined as any rating of "present" on any of the 2 items.

At T2, not only the $2 \mathrm{X}$-section items but also 5 items from the DIA-X/M-CIDI interview observational P-section were available: flat affect (P3), slow speech (P5), slow movement (P6), poverty of speech (P7) and avolition (P8). These 5 items were dichotomously rated: absent (0) versus present (1). The T2-NDC was defined as any rating of 'present' on any of the 7 items.

\section{The Positive Cluster}

Interview ratings from the DIA-X/M-CIDI psychosis G-section (G1, G2a, G3-G5, G7-G13, G13b, G14, G17, G18, G20, G20C, G21 and G22a) on delusions (14 items), hallucinations ( 5 items) and passivity ( 1 item) were collected at T2 (lifetime) and T3 (interval version). These 20 items concern classic psychotic symptoms including the cluster of Schneiderian First Rank Symptoms: persecution, conspiracy, thought interference, auditory hallucinations and passivity phenomena. Participants were first invited to read a list of all the psychotic experiences and asked whether they had ever experienced such symptoms. Each psychosis item was rated in 2 ways: absent (1) versus present (5). The 'Positive Cluster (PC) was defined as any rating of "present" on any of the 20 psychosis items (M. D. Dominguez et al., 2009).

\section{Depressive Symptoms}

At T2 and T3, the 28 symptom items of the DIA-X/M-CIDI depression E-section were used. If present for at least 2 weeks, items were rated either yes (1) or no (5). A sum score of 'Depressive Symptoms' (DS) was formed, with a minimum of 0 , and a maximum score of 28 endorsements (Krabbendam et al., 2004). 


\section{Assessment of Persistence of Symptoms Clusters}

The T2 (lifetime) and T3 (interval) ratings for PC were used to create a summary 'PC-Persistence' variable of 3 levels of persistence: never (0); once (1); and twice (2). In order to have a comparable longitudinal measure of 'NDC-Persistence', the T0 (lifetime) and T2 measures were combined into a single T0-T2 (lifetime) NDC variable. Together with the information at T3 (interval), this resulted in a summary score variable representing 3 levels of persistence: never (0); once (1); and twice (2).

\section{Assessment of Sociodemographic Characteristics and Environmental Risk Factors}

Age, gender, social status, marital status, level of education, urbanicity, cannabis exposure and self-reported trauma were considered (see Tables 1 and 2).

\section{Assessment of Antipsychotic Treatment}

As part of the CIDI treatment module, participants were shown a list of different types of medication, rating their use because of any psychopathological or psychosomatic problem. The acknowledgement of any antipsychotic medication (Q1EA4) reported at T2 and T3 was used to derive treatment ('Antipsychotic Treatment': no [0] versus yes [1]).

\section{Assessment of Clinical Relevance of Psychotic Experiences}

Clinical relevance of positive psychotic symptoms was assessed with DIA-X/MCIDI interview ratings in order to create a variable 'Psychotic Impairment' (M. D. Dominguez et al., 2009).

Three help-seeking items assessed whether participants had ever sought help because of psychotic symptoms: seeking doctors' help because of delusions (G16) or hallucinations (G23), seeking help from other mental health professionals, ranging from general practitioner or school psychologist to psychiatric sheltered housing (Q1DG). The 3 help-seeking items were rated: no (0) and yes (1). A dichotomous 'Help-seeking' variable was created indicating a positive answer to any of the 3 questions (1) versus negative answers on all (0).

The DIA-X/M-CIDI interview assesses the effect of psychotic experiences on: feeling upset, unable to work, go places or enjoy oneself, at the time of having these experiences (G28), being less able to work (G29) or less able to make friends or enjoy social relationships (G29a) since these experiences began; and how much their life and everyday activities were impaired when these experiences were at their worst (G36). These items were rated: no (0) and yes (1). A dichotomous 
'Dysfunction' variable was created representing a positive answer on any of the 4 questions (1) versus negative answers on all (0).

Finally, a variable 'Psychotic Impairment' was created, rated " 0 " in subjects with psychotic experiences who scored " 0 " on both Help-seeking and Dysfunction, and " 1 " in subjects scoring " 1 " on either or both.

\section{Caseness variable}

The X16 DIA-X/M-CIDI item rated the interviewer's opinion regarding clinical evidence of psychological ill-health in 4 levels: essentially not noticeable (0), not very noticeable (1), clearly ill (2), and very ill (3). The dichotomous variable 'Caseness' indicated individuals with a noticeable level of psychiatric caseness (any score above "1").

\section{Statistical Analyses}

All analyses were conducted using the software package STATA 10.1 (StataCorp, 2008).

First, in order to investigate the reliability and the rate of the variables representing the positive and the negative-disorganized psychopathological domains: (i) The Cronbach's alpha coefficient was used as a measure of the internal consistency for each cluster: the NDC including the 7 items (McGorry et al., 1998) and the PC including the 20 items (M. D. Dominguez et al., 2009) at T2. Alpha values of 0.7 are regarded as satisfactory; (ii) Lifetime cumulative incidence and interval prevalence estimates of both clusters were calculated. Estimates of the NDC were additionally calculated excluding participants exposed to Antipsychotic Treatment; (iii) The distribution of both cluster indicators was examined at T2 and T3 distinguishing individuals with only negative-disorganized symptoms, only positive symptoms or a combination of the two.

Second, associations between psychopathology and demographic and risk factors were examined. It was hypothesized that the NDC would be associated with younger age (Schultz et al., 1997), male sex (Leung \& Chue, 2000), single marital status and low educational level (van Os, Hanssen, Bijl, \& Ravelli, 2000), whereas female sex (Leung \& Chue, 2000), cannabis exposure (Henquet et al., 2005), urbanicity (Spauwen, Krabbendam, Lieb, Wittchen, \& van Os, 2006a), self-reported trauma (Spauwen, Krabbendam, Lieb, Wittchen, \& van Os, 2006b) and low level of education (van Os et al., 2000) would show significant associations with the PC. Logistic regression analyses were conducted with the data in the "long" format, i.e. each individual contributing two "time"-point observations (T2 and T3). Clustering within subjects was controlled for by including the variable "time" in the model. 
Associations were expressed as odds ratios (OR) and their 95\% confidence intervals (95\%CI).

Third, in order to examine the natural association between the positive and negative-disorganized clusters (McGorry et al., 1998), cross-sectional associations between the two were examined, controlling for DS. Logistic regression analyses were carried out with the data in the "long" format. Given that cross-sectional associations between symptoms may arise as a function of shared interview variance or represent chronicity effects, associations between clusters were also carried out in longitudinal predictive models across measurement occasions. Logistic regression was used to calculate: (i) the association between the T2-NDC and the T3-PC controlling for DS, and excluding individuals with presence of the $\mathrm{T} 2-\mathrm{PC}$, and (ii) the association between the T2-PC and the T3-NDC controlling for DS and excluding individuals with presence of lifetime T0-T2 NC.

Fourth, the null hypothesis of independence of the natural courses of the two psychopathological domains (Eaton et al., 1995) was examined analyzing the influence of the degree of persistence (from the interview assessing lifetime presence to T3) of one psychosis cluster (i.e. PC or NDC) on T3 onset of the other cluster and vice versa. To this end, logistic regression analyses were performed to examine the associations between: (i) the degree of NDC-Persistence on the one hand and onset of T3-PC on the other in individuals without lifetime T2-PC, (ii) the degree of NDC-Persistence on the one hand and T3-PC on the other in individuals with lifetime T2-PC; and vice versa, controlling for T3-DS.

Fifth, the clinical relevance of the combination of positive and negative experiences was calculated by examining whether the association between the PC and its associated Psychotic Impairment rating was moderated by the NDC. The interaction between the PC and the NDC was calculated on the additive scale (Henquet et al., 2005; Spauwen et al., 2006a, 2006b) using the STATA BINREG command, controlling for depression, with the data in the "long" format. An additive risk model rather than a multiplicative risk model is more likely to yield information on the degree of synergism between causes which can be interpreted biologically in a meaningful way -that is, the extent to which both causes depend on each other or co-participate in disease causation (Darroch, 1997). In order to validate Psychosis Impairment, logistic regression analyses were conducted against the Caseness and Antipsychotic Treatment variables. 


\section{Results}

\section{Subject Characteristics}

The average age of the participants at baseline was 18.3 years (range=14-24). Demographic characteristics are depicted in Table 1.

Table 1. Characteristics of Subjects at Baseline

\begin{tabular}{|c|c|c|c|}
\hline $\begin{array}{l}\text { At baseline (T0) } \\
\mathbf{n}=\mathbf{3 , 0 2 1}\end{array}$ & Variables description & $n$ & $\%$ \\
\hline \multicolumn{4}{|l|}{ Gender } \\
\hline Male & & 1,488 & 49.26 \\
\hline Female & & 1,533 & 50.74 \\
\hline $\begin{array}{l}\text { Level of } \\
\text { education }\end{array}$ & $\begin{array}{l}\text { The participants were asked which level of education } \\
\text { they were attending or, in the case of discontinuation, } \\
\text { which was the highest level they had attended (A3 } \\
\text { DIA-X/M-CIDI item). }\end{array}$ & & \\
\hline Low & - mandatory basic school or learning a profession & 475 & 15.72 \\
\hline Medium & - high school & 915 & 30.29 \\
\hline High & - high school preparing for university, and university & 1,631 & 53.99 \\
\hline Social status & $\begin{array}{l}\text { The participants were asked to choose from specified } \\
\text { options, which class he or she believed to be in (A16 } \\
\text { DIA-X/M-CIDI item). }\end{array}$ & & \\
\hline Lower & - lower class, lower middle class & 207 & 6.85 \\
\hline Middle & - middle middle class & 1,804 & 59.72 \\
\hline Upper & - higher middle class, upper class & 950 & 31.45 \\
\hline Other & - none of the above and missing values & 60 & 1.99 \\
\hline Urbanicity & $\begin{array}{l}\text { Obtained through German government population } \\
\text { registries. The population density of the Munich } \\
\text { surroundings areas was } 553 \text { persons per square mile } \\
\text { and that of the city } 4061 \text { persons per square mile }\end{array}$ & & \\
\hline Rural & - the surrounding areas of Munich & 859 & 28.43 \\
\hline Urban & - the German city of Munich & 2,162 & 71.57 \\
\hline
\end{tabular}

\section{Natural Occurrence}

Items in each psychopathological cluster were strongly correlated yielding satisfactory internal consistency (NDC items: Cronbach's $\alpha=0.7,6$ items; PC items Cronbach's $\alpha=0.7,20$ items).

The baseline cumulative lifetime incidence of NDC was $11.4 \%(n=345)$ while the interval prevalence was $12.8 \%(\mathrm{n}=325)$ at $\mathrm{T} 2$ and $12.2 \%(\mathrm{n}=270)$ at $\mathrm{T} 3$. Excluding individuals exposed to antipsychotic treatment did not impact on these rates $(12.7 \%(\mathrm{n}=322)$ at $\mathrm{T} 2$ and $12.2 \%(\mathrm{n}=269)$ at $\mathrm{T} 3)$. The $\mathrm{PC}$ cumulative lifetime incidence was $22.7 \%(\mathrm{n}=574)$ at T2 while the interval prevalence was $12.4 \%(\mathrm{n}=274)$ at T3. 
In terms of population distribution (Table 2), 15.7\% of the 2548 participants reported only negative-disorganized symptoms at T2 (lifetime cumulative incidence), $17 \%$ reported only positive symptoms whereas $5.5 \%$ reported both, more than twice what would be expected by chance alone (2.6\%). At T3 (interval), prevalence rates were, respectively, $9.9 \%, 10.1 \%$ and $2.3 \%$ of a total of 2210 participants, again more than twice the expected rate $(1 \%)$.

\section{Associations with Sociodemographic and Environmental Risk Factors}

The NDC was significantly associated with younger age, male sex, single marital status and low educational level, whereas the PC was significantly associated with low educational level, cannabis exposure, urbanicity and self-reported trauma. The combination of both clusters was significantly associated with all variables (Table 3 ).

\section{Associations between Psychopathological Clusters}

Cross-sectionally, the NDC was significantly associated with the PC, controlling for $\mathrm{DS}(\mathrm{OR}=1.36,95 \% \mathrm{CI}=1.10-1.69)$. The prospective association between the $\mathrm{T} 2$ NDC and the T3-PC, excluding individuals with presence of lifetime T2-PC and controlling for $\mathrm{DS}$, was significant $(\mathrm{OR}=4.65,95 \% \mathrm{CI}=1.09-19.96)$. In contrast, the prospective association between the T2-PC and the T3-NDC, excluding individuals with presence of lifetime T0-T2 NC and controlling for DS, was neither large nor significant $(\mathrm{OR}=0.82,95 \% \mathrm{CI}=0.55-1.24)$.

\section{Independence of Natural Course}

Controlling for depressive symptoms, NDC-Persistence not only predicted PCPersistence but also T3 incident psychotic experiences in a dose-response fashion. In contrast, PC-Persistence predicted neither NDC-Persistence nor T3 incident negative-disorganized symptoms (Table 4).

\section{Psychopathological Clusters and Clinical Relevance}

Psychotic Impairment was strongly associated with the Caseness $(\mathrm{OR}=10.3$, $95 \% \mathrm{CI}=7.0-15.2)$ and the Antipsychotic Treatment $(\mathrm{OR}=15.3,95 \% \mathrm{CI}=6.1-38.4)$ variables.

Proportion of T2 and T3 Psychotic Impairment with expression of psychosis clusters are depicted in Table 2. Differences in risk for Psychotic impairment for each psychopathological cluster were statistically significant in the combined T2 and T3 data analysis, as evidenced by the estimated additive interaction between the two clusters in the model of impairment $(\mathrm{RD}=16.2 \%, 95 \% \mathrm{CI}=7.3 \%-25.2 \%)$. Analysing T2 and T3 separately revealed replication at both time points (Table 2). 


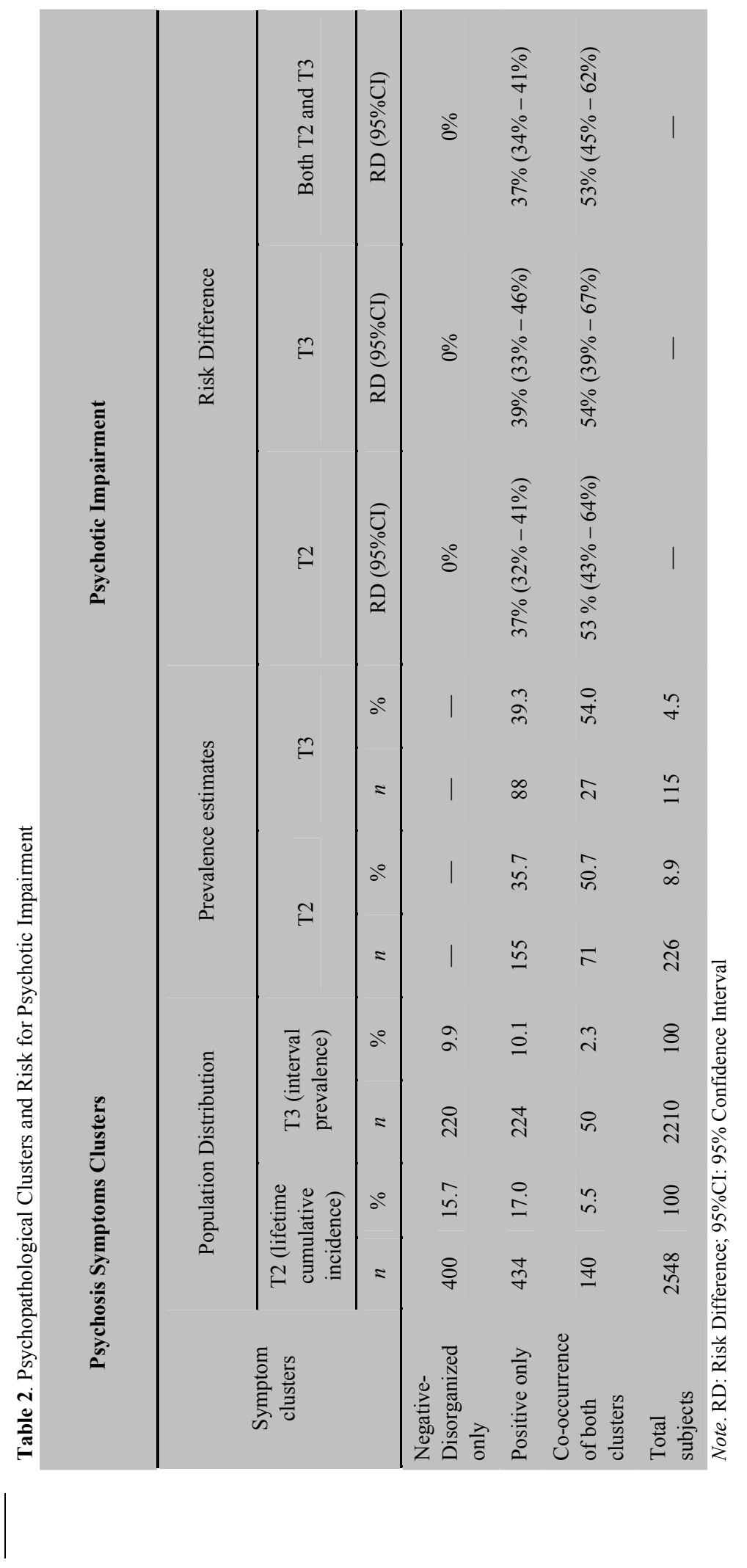




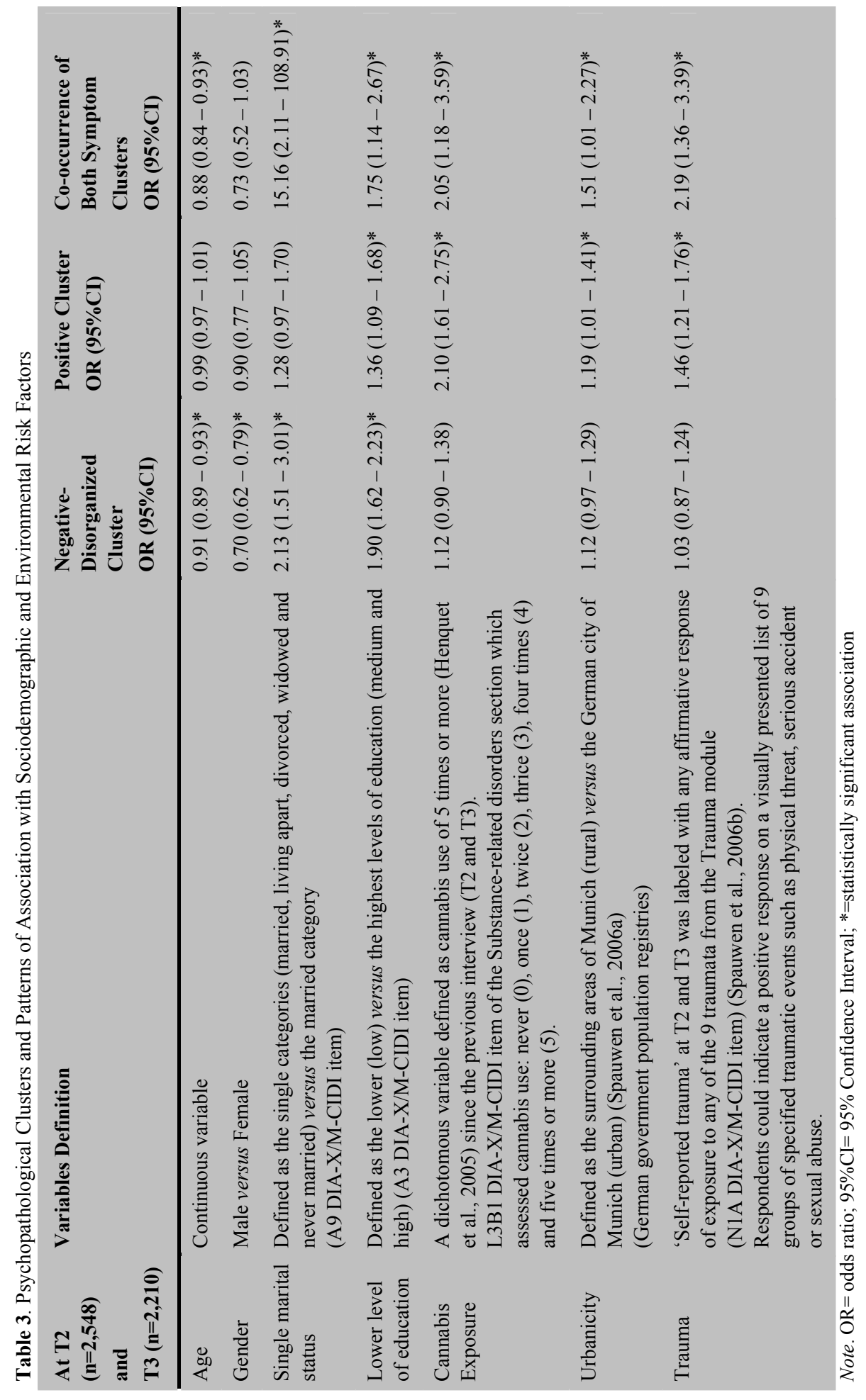


Table 4. Longitudinal Association of Natural Courses

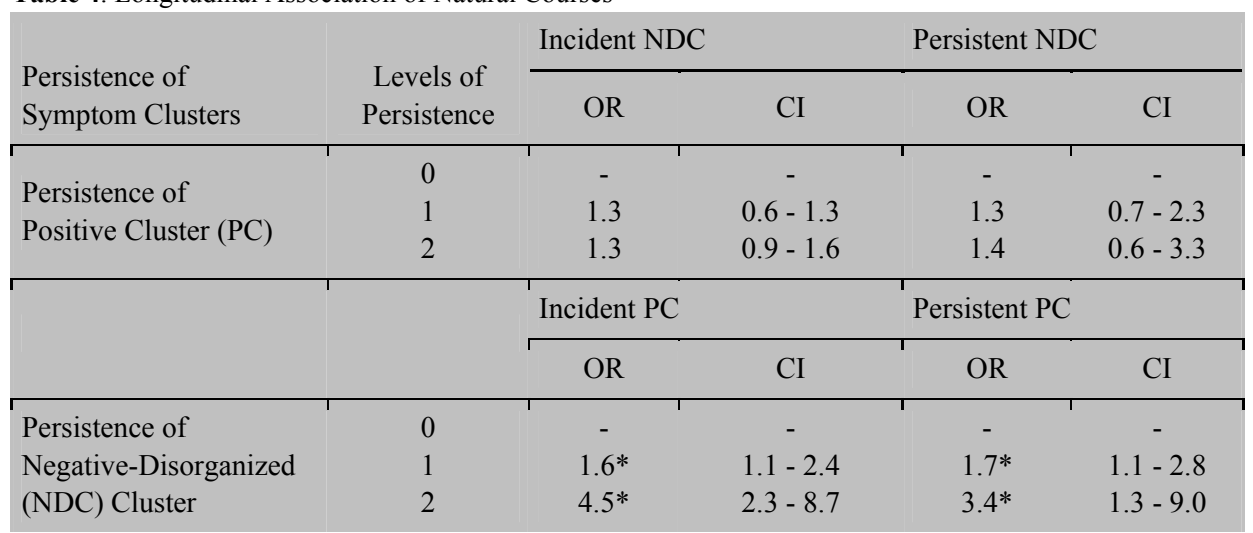

Note. $\mathrm{OR}=$ odds ratio $\mathrm{CI}=95 \%$ Confidence Interval; $*=$ statistically significant association

\section{Discussion}

\section{Findings}

Experiences indicative of the positive (van Os et al., 2009) and negative symptoms seen in psychotic disorders occurred in the general population (5-year interval rates of around 12\%), independent of antipsychotic treatment. Positive and negative dimension scores clustered together more often than predicted by chance, independent of depression, yet they displayed a differential pattern of sociodemographic and environmental risk factors associations: the NDC was associated with a profile indicative of developmental impairment and the PC with environmental risks. Their pattern over time revealed that persistent expression of the NDC predicted onset of psychotic experiences; in contrast, persistent expression of psychotic experiences did not predict onset of negative-disorganized features. Finally, "comorbid" expression of the two clusters increased the risk for impairment and, by implication, need for care.

\section{Quantitative Variations of Normal Human Mentation}

Self-reported negative-disorganized and positive psychotic features were distributed in the general population suggesting they represent quantitative variation of processes underlying normal human mentation. While dopamine-blocking medications dampen motivational drives causing secondary negative symptoms (Kapur, 2004) in both clinical (Carpenter, Heinrichs, \& Alphs, 1985) and healthy samples (Artaloytia et al., 2006), this study highlighted the natural occurrence of attenuated negative symptoms in otherwise healthy and unmedicated individuals. The hypothesis of an exclusively drug-induced nature of non-motor negative symptoms (Artaloytia et al., 2006) therefore is unlikely. 


\section{Negative Features Predict Positive Features and Onset of Clinical Psychosis}

It has been suggested that cognitive/negative symptoms associated with schizophrenia may represent alterations in brain development associated with distributed genetic risk, influencing a final common pathway of neurotransmitter dysregulation resulting in the onset of positive psychotic symptoms, particularly when combined with environmental exposures (van Os \& Kapur, 2009). Several elements in the current epidemiological analysis support this model.

First, the PC and NDC clustered together more often than could be expected by chance alone. Thus, psychotic disorders such as schizophrenia are not the result of the chance cross-section of the separate liabilities of developmental impairment on the one hand and reality distortion on the other; they are linked together in a way that suggests fundamental overlap or interaction of underlying mechanisms.

Second, the pattern of associations for the NDC with young age, male sex, single marital status and lower educational level resembles the pattern seen in clinical samples (Leung \& Chue, 2000; Schultz et al., 1997). The psychotic NDC, in contrast to the PC, is associated with neurocognitive impairment (M. D. G. Dominguez et al., 2009) resulting in poorer social functioning (Macdonald et al., 2000), poorer response to antipsychotic treatment (Erhart et al., 2006) and a worse prognosis (McGlashan \& Fenton, 1992).

Third, the socio-demographic associations of the PC are in agreement with previous reports linking positive psychotic experiences to environmental risk factors such as cannabis exposure, urbanicity, self-reported trauma and lower educational level (Henquet et al., 2005; Spauwen et al., 2006a, 2006b; van Os et al., 2000). The link between these environmental risk factors and psychotic experiences may be explained in terms of stress-dopamine (DA) interactions and sensitization (Collip, Myin-Germeys, \& Van Os, 2008). There is evidence that mesocortical DAergic innervation of the prefrontal cortex may regulate the activity of mesolimbic subcortical DA innervations and that the impact of environmental risk factors may result in taking the prefrontal cortical (PFC) "off-line", resulting in altered responsiveness of subcortical DAergic innervations (Deutch, Clark, \& Roth, 1990). Thus, when DA transmission is increased by exposure to stress or agonist drugs, there may be a shift in the balance of the system away from PFC control and toward limbic predominance, facilitating a DA hyperactivity in the ventral tegmental area of the midbrain, resulting in the development of positive symptoms (Laruelle et al., 2003).

Fourth, importantly, the longitudinal analysis suggested that in this community sample negative features preceded positive features rather than the other way round. 
This, together with the associations between positive features and environmental risks, suggests that the NDC reflects an underlying vulnerability that, when combined with environmental exposures, results in positive symptoms increasing the risk of need for care (Figure 1). This latter suggestion is supported by the fact that the combination of positive and negative features predicted impairment and onset of clinical psychosis.

Figure 1. Negative/disorganized Features Predict Positive Features and Onset of Clinical Psychosis

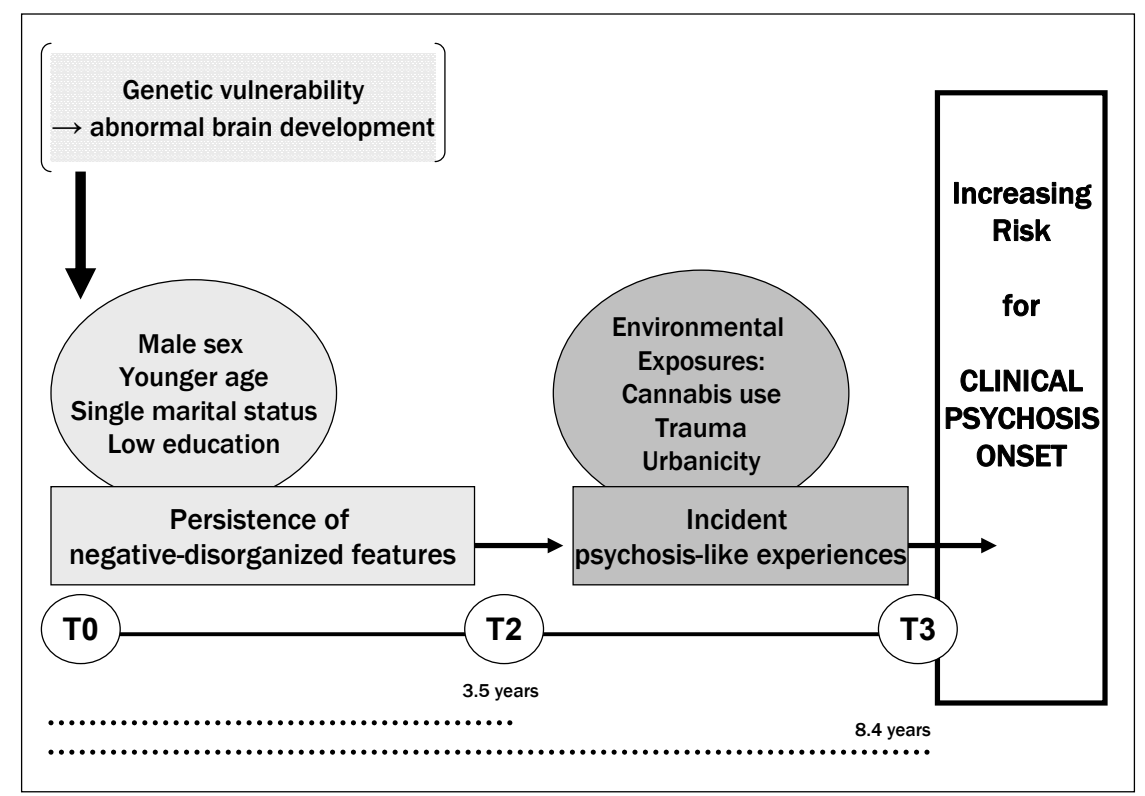

Note. The longitudinal analysis suggested that the negative-disorganized cluster associated with a sociodemographic indicative of developmental impairment reflects an underlying vulnerability that, when combined with environmental exposures such as cannabis use, trauma and urbanicity, results in positive psychotic symptoms increasing the risk of impairment and clinical relevance.

\section{Methodological Issues}

First, assessment of psychotic experiences was based on self-report information by the respondents in diagnostic interviews, complemented by clinical ratings of core features of positive and negative symptoms derived from the clinical rating section and selected BPRS items. False-positive psychotic experiences are likely to have occurred, increasing random error; nevertheless, there is a substantial literature on the (predictive and other forms of) validity of self-reported psychotic experiences (van Os et al., 2009). 
Second, despite the hazard of under/overestimation of true positive, clinically relevant psychotic experiences (M. D. Dominguez et al., 2009), the validation of the outcome variable against Caseness and Antipsychotic Treatment supports the validity of Psychotic Impairment as a proxy measure of clinical relevance.

Third, the definition of clinically relevant psychosis was contingent on the presence of positive psychotic experiences. This, however, is in line with the diagnostic criteria for schizophrenia, which require positive psychotic experiences for making the diagnosis.

Fourth, the measures of negative symptoms were varying between measurement occasions. This is likely to have generated more random error resulting in a more conservative estimate of associations (i.e., the overlap between PC and NDC may be stronger than reported and the predictive value for positive psychotic experiences may be higher).

Finally, our measure of negative symptoms included features of disorganization in accordance with first-episode studies (McGorry et al., 1998) while studies using more chronic samples reported a separate disorganized dimension (Liddle, Carpenter, \& Crow, 1994). Given that the current sample was well before the earliest possible phase of psychotic illness onset, combining negative and disorganized features in one domain appeared the most parsimonious solution. Also, a recent meta-analysis of studies examining associations between psychotic psychopathology and neurocognition reported associations between neurocognition and both the negative and disorganized dimensions, but not the psychotic and affective dimensions (M. D. G. Dominguez et al., 2009).

\section{Acknowledgments}

Funding/Support: This work is further part of the Early Developmental Stages of Psychopathology (EDSP) Study and is supported by the German Federal Ministry of Education and Research (BMBF) project no. 01EB9405/6, 01EB9910/6, EB10106200, 01EB0140, and 01EB0440. Part of the field work and analyses were also additionally supported by grants of the Deutsche Forschungsgemeinschaft (DFG) LA1148/1-1, WI2246/7-1, and WI709/8-1.

Data Access and Responsibility: Principal investigators of the EDSP-Study are Dr. Hans-Ulrich Wittchen and Dr. Roselind Lieb. Both take responsibility for the integrity of the study data. All authors and co-authors had full access to all study data. Data analysis and manuscript preparation were completed by the authors and co-authors of this article, who take responsibility for its accuracy and content. 
Acknowledgements: Core staff members of the EDSP group are: Dr Katja Beesdo, Dr. Petra Zimmermann, Dr. Axel Perkonigg, PhD Dipl.-Stat. Michael Höfler, , Dipl.-Psych. Tanja Brückl, Dipl.-Psych. Agnes Nocon, Dipl.-Inf. Hildegard Pfister, Dipl.-Soz. Barbara Spiegel, Dipl.-Psych. Andrea Schreier. Scientific advisors are Dr. Jules Angst (Zurich), Dr. Kathleen Merikangas (NIMH, Bethesda), Dr. Ron Kessler (Harvard, Boston) and Dr. Jim van Os (Maastricht).

\section{References}

Artaloytia, J. F., Arango, C., Lahti, A., Sanz, J., Pascual, A., Cubero, P., et al. (2006). Negative signs and symptoms secondary to antipsychotics: a double-blind, randomized trial of a single dose of placebo, haloperidol, and risperidone in healthy volunteers. Am J Psychiatry, 163(3), 488-493.

Carpenter, W. T., Jr., Heinrichs, D. W., \& Alphs, L. D. (1985). Treatment of negative symptoms. Schizophr Bull, 11(3), 440-452.

Chapman, L. J., Chapman, J. P., Kwapil, T. R., Eckblad, M., \& Zinser, M. C. (1994). Putatively psychosisprone subjects 10 years later. J Abnorm Psychol, 103(2), 171-183.

Collip, D., Myin-Germeys, I., \& van Os, J. (2008). Does the concept of "sensitization" provide a plausible mechanism for the putative link between the environment and schizophrenia? Schizophr Bull, 34(2), 220-225

Craddock, N., \& Owen, M. J. (2007). Rethinking psychosis: the disadvantages of a dichotomous classification now outweigh the advantages. World Psychiatry, 6(2), 84-91.

Darroch, J. (1997). Biologic synergism and parallelism. Am J Epidemiol, 145(7), 661-668.

Deutch, A. Y., Clark, W. A., \& Roth, R. H. (1990). Prefrontal cortical dopamine depletion enhances the responsiveness of mesolimbic dopamine neurons to stress. Brain Res, 521(1-2), 311-315.

Dominguez, M. D., Wichers, M., Lieb, R., Wittchen, H. U., \& van Os, J. (2009). Evidence That Onset of Clinical Psychosis Is an Outcome of Progressively More Persistent Subclinical Psychotic Experiences: An 8-Year Cohort Study. Schizophr Bull.

Dominguez, M. D. G., Viechtbauer, W., Simons, C. J. P., van Os, J., \& Krabbendam, L. (2009). Are Psychotic Psychopathology and Neurocognition Orthogonal? A systematic Review of their Associations. Psychological Bulletin, 135(1), 157-171.

Eaton, W. W., Thara, R., Federman, B., Melton, B., \& Liang, K. Y. (1995). Structure and course of positive and negative symptoms in schizophrenia. Arch Gen Psychiatry, 52(2), 127-134.

Erhart, S. M., Marder, S. R., \& Carpenter, W. T. (2006). Treatment of schizophrenia negative symptoms: future prospects. Schizophr Bull, 32(2), 234-237.

Hanssen, M., Bak, M., Bijl, R., Vollebergh, W., \& van Os, J. (2005). The incidence and outcome of subclinical psychotic experiences in the general population. Br J Clin Psychol, 44(Pt 2), 181-191.

Henquet, C., Krabbendam, L., Spauwen, J., Kaplan, C., Lieb, R., Wittchen, H. U., et al. (2005). Prospective cohort study of cannabis use, predisposition for psychosis, and psychotic symptoms in young people. Bmj, 330(7481), 11.

Kapur, S. (2004). How antipsychotics become anti-"psychotic"--from dopamine to salience to psychosis. Trends Pharmacol Sci, 25(8), 402-406.

Kendler, K. S., McGuire, M., Gruenberg, A. M., Spellman, M., O’Hare, A., \& Walsh, D. (1993). The Roscommon Family Study. II. The risk of nonschizophrenic nonaffective psychoses in relatives. Arch Gen Psychiatry, 50(8), 645-652.

Krabbendam, L., Myin-Germeys, I., De Graaf, R., Vollebergh, W., Nolen, W. A., Iedema, J., et al. (2004). Dimensions of depression, mania and psychosis in the general population. Psychol Med, 34(7), 11771186.

Laruelle, M., Kegeles, L. S., \& Abi-Dargham, A. (2003). Glutamate, dopamine, and schizophrenia: from pathophysiology to treatment. Ann N Y Acad Sci, 1003, 138-158.

Lataster, T., Myin-Germeys, I., Derom, C., Thiery, E., \& van Os, J. (2009). Evidence that self-reported psychotic experiences represent the transitory developmental expression of genetic liability to psychosis in the general population. Am J Med Genet B Neuropsychiatr Genet. 
Leung, A., \& Chue, P. (2000). Sex differences in schizophrenia, a review of the literature. Acta Psychiatr Scand Suppl, 401, 3-38.

Liddle, P., Carpenter, W. T., \& Crow, T. (1994). Syndromes of schizophrenia. Classic literature. $\mathrm{Br} J$ Psychiatry, 165(6), 721-727.

Lieb, R., Isensee, B., von Sydow, K., \& Wittchen, H. U. (2000). The Early Developmental Stages of Psychopathology Study (EDSP): a methodological update. Eur Addict Res, 6(4), 170-182.

Macdonald, E. M., Hayes, R. L., \& Baglioni, A. J., Jr. (2000). The quantity and quality of the social networks of young people with early psychosis compared with closely matched controls. Schizophr Res, 46(1), 2530.

Malla, A. K., Takhar, J. J., Norman, R. M., Manchanda, R., Cortese, L., Haricharan, R., et al. (2002). Negative symptoms in first episode non-affective psychosis. Acta Psychiatr Scand, 105(6), 431-439.

Maric, N., Myin-Germeys, I., Delespaul, P., de Graaf, R., Vollebergh, W., \& van Os, J. (2004). Is our concept of schizophrenia influenced by Berkson's bias? Soc Psychiatry Psychiatr Epidemiol, 39(8), 600-605.

McGlashan, T. H., \& Fenton, W. S. (1992). The positive-negative distinction in schizophrenia. Review of natural history validators. Arch Gen Psychiatry, 49(1), 63-72.

McGorry, P. D., Bell, R. C., Dudgeon, P. L., \& Jackson, H. J. (1998). The dimensional structure of first episode psychosis: an exploratory factor analysis. Psychological Medicine, 28(4), 935-947.

Overall, J. E., \& Gorham, D. R. (1962). The brief psychiatric rating scale. Psychol Rep, 10, 799-812.

Poulton, R., Caspi, A., Moffitt, T. E., Cannon, M., Murray, R., \& Harrington, H. (2000). Children's selfreported psychotic symptoms and adult schizophreniform disorder: a 15-year longitudinal study. Arch Gen Psychiatry, 57(11), 1053-1058.

Reed, V., Gander, F., \& Pfister, H. (1998). To what degree does the Composite International Diagnostic Interview (CID) correctly identify DSM-IV disorders? Testing validity issues in a clinical sample. International Journal of Methods in Psychiatric Research, 7, 142-155.

Schroder, J., Buchsbaum, M. S., Siegel, B. V., Geider, F. J., Lohr, J., Tang, C., et al. (1996). Cerebral metabolic activity correlates of subsyndromes in chronic schizophrenia. Schizophrenia Research, 19(1), 41-53.

Schultz, S. K., Miller, D. D., Oliver, S. E., Arndt, S., Flaum, M., \& Andreasen, N. C. (1997). The life course of schizophrenia: Age and symptom dimensions. Schizophrenia Research, 23(1), 15-23.

Spauwen, J., Krabbendam, L., Lieb, R., Wittchen, H. U., \& van Os, J. (2006a). Evidence that the outcome of developmental expression of psychosis is worse for adolescents growing up in an urban environment. Psychol Med, 36(3), 407-415.

Spauwen, J., Krabbendam, L., Lieb, R., Wittchen, H. U., \& van Os, J. (2006b). Impact of psychological trauma on the development of psychotic symptoms: relationship with psychosis proneness. $\mathrm{Br} J$ Psychiatry, 188, 527-533.

van Os, J., Hanssen, M., Bijl, R. V., \& Ravelli, A. (2000). Strauss (1969) revisited: a psychosis continuum in the general population? Schizophr Res, 45(1-2), 11-20.

van Os, J., \& Kapur, S. (2009). Schizophrenia. Lancet, 374(9690), 635-645.

van Os, J., Linscott, R. J., Myin-Germeys, I., Delespaul, P., \& Krabbendam, L. (2009). A systematic review and meta-analysis of the psychosis continuum: evidence for a psychosis proneness-persistenceimpairment model of psychotic disorder. Psychol Med, 39(2), 179-195.

Welham, J., Scott, J., Williams, G., Najman, J., Bor, W., O’Callaghan, M., et al. (2009). Emotional and behavioural antecedents of young adults who screen positive for non-affective psychosis: a 21-year birth cohort study. Psychol Med, 39(4), 625-634.

Werbeloff, N., Drukker, M., \& Dohrenwend, B. (2009). Self-Reported Psychotic Symptoms in the Community are Associated with Increased Risk of Later Hospitalization for Non-Affective Psychotic Disorders. In C. Abstract (Ed.) (Vol. 35, pp. 74): Schizophrenia Bulletin.

Wittchen, H. U. (1994). Reliability and validity studies of the WHO--Composite International Diagnostic Interview (CIDI): a critical review. J Psychiatr Res, 28(1), 57-84.

Wittchen, H. U., \& Pfister, H. (1997). DIS-X-Interviews: Manual für Screening-Verfahren und Interview; Interviewheft Längsschnittuntersuchung (DIA-X-Lifetime); Ergänzungsheft (DIA-X-Lifetime); Interviewheft Querschnittsuntersuchung (DIA-X-12 Monats-Version); Ergänzungsheft (DIA-X-12 Monats-Version); PC-Programm zur Durchführung der Interviews (Längsund Querschnittsuntersuchung). Auswertungsprogramm. Frankfurt: Swets \& Zeitlinger. . 
Wittchen, H. U., Robins, L. N., Cottler, L. B., Sartorius, N., Burke, J. D., \& Regier, D. (1991). Cross-cultural feasibility, reliability and sources of variance of the Composite International Diagnostic Interview (CIDI). The Multicentre WHO/ADAMHA Field Trials. Br J Psychiatry, 159, 645-653, 658. 


\title{
CHAPTER 6
}

\author{
DISCUSSION
}




\section{DISCUSSION : CONTENTS}

\section{Quantitative Variations of Normal Human Mentation}

\section{The Psychosis Proneness-Persistence-Impairment Model}

From Persistent Subclinical Psychosis to Impairment: Possible Behavioural, Environmental and Neurobiological Interactions

\section{Understanding How Interacting Processes Underlying Psychopathological}

\section{Dimensions Contribute to the Onset of Clinical Psychosis}

The Cluster of Disorganized and Negative Symptoms versus Positive and Affective Ones in Psychotic Disorders

Different Underlying Pathophysiological Processes Associated with Different Intermediary Phenotypes May Account for a Substantial Part of Psychopathological Heterogeneity

Integrating Affective Dysregulation and Reality Distortion from a Perspective of Risk

Negative and Disorganized Features Increasing Risk for Positive Symptoms Increasing Risk for Impairment

A Dynamic Model of the Onset of Clinical Psychosis from an Epidemiological Perspective

Nosological Implications

Clinical Implications

Directions for Future Research 
Delusions and hallucinations are not exclusive to schizophrenia and psychotic symptoms occur across a wide range of diagnostic categories with their differential diagnosis being based on duration, symptoms patterns, level of dysfunction, substance use, "bizarreness" of delusions and presence of depression or mania. However, diagnostic categories show overlap in their genetic liability (Kendler \& Diehl, 1993), suggesting a shared genetic liability across non affective psychosis and bipolar disorder (Craddock \& Owen, 2007). A consilience model of clinical psychosis (van Os \& Kapur, 2009) postulates that observed phenomenological heterogeneity of psychosis is associated with different genetic liabilities: (i) positive psychotic symptoms, shared by all psychotic diagnostic categories, occupy a central position in the model, and plausibly result from gene-environment interactions; (ii) social-interpersonal style and negative symptoms, associated with neurocognitive impairment, may be the phenomenological expression of altered brain development; and (iii) affective genetic risk may lead to the expression of positive psychotic symptoms or reality distortion through the mechanism of aberrance salience attribution.

Psychotic experiences have been shown to be expressed at levels well below its clinical manifestation. A systematic review of general population surveys suggests that phenomena indicative of schizophrenia and related disorders -e.g. persecutory delusions and auditory hallucinations- are observed it in an attenuated form, in around $5-8 \%$ of healthy people (van Os, Linscott, Myin-Germeys, Delespaul, \& Krabbendam, 2009). These attenuated expressions may be the behavioural expression of an underlying continuous psychosis liability (Lataster, MyinGermeys, Derom, Thiery, \& van Os, 2009). This is further supported by longitudinal studies demonstrating a link between psychotic experiences in the general population and increased probability of having a psychotic disorder later in life (Chapman, Chapman, Kwapil, Eckblad, \& Zinser, 1994; Hanssen, Bak, Bijl, Vollebergh, \& van Os, 2005; Poulton et al., 2000; Welham et al., 2009; Werbeloff, Drukker, \& Dohrenwend, 2009). To date, no published study has clearly demonstrated abnormal persistence of (normally transitory) developmental subclinical psychosis like symptoms to be the precursor state of clinical presentation. Furthermore, the population-surveys have focused on positive psychotic-like experiences only, ignoring if and how another attenuated symptom domains may play a role driving subclinical psychosis expression to clinical states. Therefore, population-based research on such psychopathological phenomena may be productive, particularly when a longitudinal perspective allows for the epidemiological prediction of the model of clinical psychosis.

The studies described in this thesis aim to investigate the evolution of psychosis from a starting position of mental health to the onset of clinical psychosis within a large representative sample of adolescents in the general population (the Early 
Developmental Stages of Psychopathology (EDSP) study; (Lieb, Isensee, von Sydow, \& Wittchen, 2000; Wittchen, Perkonigg, Lachner, \& Nelson, 1998)) who were followed up for maximum of 10 years, by examining (i) the quantitative variation of psychometric expressions of the psychosis phenotype (chapters 3, 4 and 5), (ii) the hypothesis of continuity over time from abnormal persistence of commonly transitory psychotic-like experiences to clinically relevant psychosis (chapter 3), and (iii) if and how interacting processes underlying psychopathological dimensions (the associations of which were obtained by a metaanalysis of associations between psychosis dimensions and the intermediary phenotype of neurocognition (chapter 2)), from a perspective of risk, may contribute to the onset of clinical psychosis (chapter 4 and 5).

\section{Quantitative Variation of Normal Human Mentation}

Self-reported psychopathological experiences resembling the positive psychotic, negative-disorganized, depressive and manic symptoms are also expressed in attenuated form in otherwise "healthy" individuals (Chapman et al., 1994; Hanssen et al., 2005; Krabbendam et al., 2004; Krabbendam, Myin-Germeys, Hanssen et al., 2005; Maric et al., 2004; Poulton et al., 2000; Reed, Gander, \& Pfister, 1998; van Os, Hanssen, Bijl, \& Ravelli, 2000) (chapters 3, 4 y 5). Attenuated expressions of psychosis symptom domains therefore seem to be distributed in the general population to the degree that they represent quantitative variations of normal human mentation.

In the EDSP study, subclinical psychosis was confirmed to be relatively common (Psychosis Expression rates of 22\%) and mostly transitory, recurrence or persistence occurring in $30-40 \%$ of cases (chapter 3). The recurrence-persistence rate of subclinical psychosis in our study was higher than reported in previous population-based studies. In a two-year follow-up of a general population sample aged 18-64 years, Hanssen and colleagues found a two-year 8\% persistence rate of baseline subclinical psychosis, and an $8 \%$ transition rate from subclinical to clinical psychosis (Hanssen et al., 2005). In our study, the recurrence-persistence rate was $30-40 \%$ over time, considerably higher. The most likely factor to explain this difference is the much younger age of the EDSP sample. Given the rapid decline of psychosis expression with age (Peters, Joseph, \& Garety, 1999; van Os et al., 1999; Verdoux et al., 1998), continuity over time will become less likely with advancing age. Thus, in a previous follow-up study of adolescents, a 3-year persistence rate was found of $40 \%$, similar to that of the current study (Escher, Romme, Buiks, Delespaul, \& van Os, 2002).

Affective symptoms in our study have also been shown to be expressed at levels well below its clinical manifestation (chapter 4) existing along a spectrum including 
subclinical expressions, evidenced by relatively high rates of expression of bipolar spectrum symptoms and subthreshold depressive states (Akiskal \& Vazquez, 2006; Angst \& Cassano, 2005).

Experiences indicative of negative symptoms seen in psychotic disorders also occurred in the EDSP population sample (5-year cumulative prevalence rates of around $12 \%$ ), independent of antipsychotic treatment (chapter 5). While dopamineblocking medications dampen motivational drives causing secondary negative symptoms (Kapur, 2004) in both clinical (Carpenter, Heinrichs, \& Alphs, 1985) and healthy samples (Artaloytia et al., 2006), the present work highlighted the natural occurrence of attenuated negative symptoms in otherwise healthy and unmedicated individuals. The hypothesis of an exclusively drug-induced nature of non-motor negative symptoms (Artaloytia et al., 2006) therefore is unlikely.

\section{The Psychosis Proneness-Persistence-Impairment Model}

Longitudinal cohort studies have consistently shown an association between subclinical psychotic experiences and increased likelihood of developing a psychotic disorder later in life (Chapman et al., 1994; Poulton et al., 2000; Welham et al., 2009)(Hanssen et al., 2005; Werbeloff et al., 2009). Population-based studies demonstrate that subclinical delusional-like thinking and mild hallucinatory experiences in the general population are usually transitory (Chapman et al., 1994; Hanssen et al., 2005; Poulton et al., 2000), but when combined with exposure to additional environmental risks during critical developmental periods, e.g cannabis use, childhood trauma and urban environment (Cougnard et al., 2007) the usually transitory phenomena become abnormally persistent and are more likely to progress to a clinical psychotic state.

Chapter 3 (M.D.G. Dominguez, Wichers, Lieb, Wittchen, \& van Os, 2009) presents the first study to epidemiologically describe the evolution of transitory psychosis-like symptoms to the development of abnormal persistence increasing the probability of clinical deterioration. In the study described in this thesis, subclinical psychosis was confirmed to be common (Psychosis Expression rates of 22\%) and mostly transitory. Persistent positive symptoms at a subclinical level posed a significant risk for clinically relevant psychosis over time and thus can be considered a marker of emerging clinical states. An exposure gradient was found with longer duration of (more persistent) subclinical psychosis carrying a greater risk of impairment. Although most subclinical psychosis was transitory, this study demonstrates the impact of the relationship between the clinical and the subclinical is not negligible: around $40 \%$ of new onset, clinically relevant psychosis in young adults was associated with subclinical psychosis ten years earlier, and furthermore one fifth displayed recurrent or persistent subclinical psychosis. Therefore for some 
individuals, psychosis is the result of a long process manifest by subclinical experiences and functional deficits. Two consequences arise from these findings: first, positive psychotic experiences can be regarded as the behavioural expression of underlying distributed vulnerability for psychosis and, second, a significant proportion of psychotic disorder may be conceptualised as the rare poor outcome of a more common developmental phenotype characterised by persistence of psychometrically detectable subclinical psychotic experiences. Consequently, these findings provide evidence for the continuity over time from subclinical persistent psychotic experiences to clinical psychotic states, yielding insight into the dynamics preceding the clinical onset of psychotic disorder from a population perspective; this has been summarised descriptively as a Psychosis Proneness-PersistenceImpairment model of psychotic disorder (M.D.G. Dominguez et al., 2009; van Os et al., 2009).

\section{From Persistent Subclinical Psychosis to Impairment: Behavioural, Environmental and Neurobiological Interactions}

The positive psychosis pathway may be understood as the phenomenological correlate of the classical dopamine hypothesis which proposes that hyperactivity of dopamine (DA) transmission from DA cell bodies located in the ventral tegmental area of the midbrain to their terminal fields in the nucleus accumbens and limbic cortex results in the development of positive psychotic symptoms (Laruelle, 2000; Lieberman, Sheitman, \& Kinon, 1997). Dopaminergic hyperactivity may be associated with what has been called a state of endogenous sensitization (Laruelle, 2000). The liability for psychosis may involve deficits in neural regulation resulting in a pathological condition of neurochemical sensitization of the mesolimbic dopaminergic system analogous to the preclinical model of behavioral sensitization induced by administration of dopamine releasing drugs (Lieberman et al., 1997). Behavioral sensitization is a progressive, enduring enhancement of behavior that develops following repeated stimulant administration. It is mediated in part by dopaminergic pathways that are also thought to modulate the development of psychosis (Wolf, White, Nassar, Brooderson, \& Khansa, 1993).

Endogenous neurochemical sensitization may arise in part as a result of exposure to environmental conditions (Laruelle, Kegeles, \& Abi-Dargham, 2003). For example, environmental stress can induce sensitization and has been demonstrated to be cross-reactive with many types of pharmacologically induced sensitization, including dopamine and opiate agonists (Lieberman et al., 1997). It has been suggested that several environmental risk factors, e.g., perinatal injury, childhood trauma, repeated life events and drug abuse, may contribute to dopamine sensitization (Collip, Myin-Germeys, \& van Os, 2008). It is attractive to speculate that a genetic tendency to abnormal dopamine sensitization is associated with the 
tendency to express low grade psychotic experiences that in most individuals will only be expressed transitorily over development. However, in the case of developmental exposure to environmental risk factors, individuals may develop a sensitized dopamine system resulting in abnormally persistent (Cougnard et al., 2007) and, eventually, clinically relevant psychotic states (M.D.G. Dominguez et al., 2009).

\section{Understanding How Interacting Processes Underlying Psychopathological Dimensions Contribute to the Onset of Clinical Psychosis}

Epidemiological research on psychosis at the level of the general population has mainly focused on positive psychotic-like experiences. Although the pool of symptom dimensions from the psychosis phenotype has also been observed at a subclinical level (Krabbendam et al., 2004; Krabbendam, Myin-Germeys, Hanssen et al., 2005; Maric et al., 2004; Stefanis et al., 2002), how development of the various underlying symptom domains contribute to the risk of clinical psychosis has not been investigated.

\section{The Cluster of Disorganized and Negative Symptoms versus Positive and Affective Ones in Psychotic Disorders}

Previous published work has not been able to define the pattern of intercorrelations of psychosis dimensions either at a population-level or in clinical samples (chapter 1). Hundreds of studies have examined the association between psychotic psychopathology and neurocognitive intermediary phenotypes but their findings have been inconsistent .Therefore, chapter 2 (M. D. G. Dominguez, Viechtbauer, Simons, van Os, \& Krabbendam, 2009) describes a meta-analysis of psychosis dimensions (including affective and non-affective measures of psychopathology) and the intermediary phenotype of neurocognition (considering all neurocognitive measures) in subjects with a lifetime history of non-affective psychosis. Furthermore the patterns of inter dimensional correlation were examined.

The systematic review (58 studies, 5009 individuals) concluded that psychopathological heterogeneity was weakly but differentially related to distinct patterns of neurocognitive function: negative and disorganized dimensions were modestly but meaningfully associated with neurocognitive impairment in the majority of neurocognitive domains whereas positive and depressive dimensions were not Associations were in the expected direction but small to moderate in magnitude (correlations ranging from -0.29 to -0.12 ). In addition, significantly higher correlations were found for the negative dimension in relation to verbal fluency (p-value: 0.005), and for the disorganized dimension in relation to reasoning and problem solving (p-value: 0.004 ), and attention/vigilance ( $\mathrm{p}$-value: 
0.03). These results further suggest that distinguishing multiple domains of cognitive functioning in schizophrenia is useful, even though the deficit is to a large extent generalized and may even be accounted for statistically by a single-factor model (as suggested by the CATIE trial, (Keefe et al., 2006)). Furthermore, the patterns of association for the four psychosis dimensions were stable across neurocognitive domains and independent of age, gender and chronicity of illness. Thus, cognitive impairment in psychosis (Aleman, Hijman, de Haan, \& Kahn, 1999; Fioravanti, Carlone, Vitale, Cinti, \& Clare, 2005; Heinrichs \& Zakzanis, 1998; Johnson Selfridge \& Zalewski, 2001; Lee \& Park, 2005; Pelletier, Achim, Montoya, Lal, \& Lepage, 2005) does not appear to be entirely orthogonal to psychopathology, as heterogeneity in non-affective psychosis is weakly but meaningfully associated with measures of neurocognition. Differential patterns of cognitive performance among the different psychopathological indicated some meaningful contrasts, suggesting differential latent cerebral mechanisms underlying the cluster of disorganized and negative symptoms on the one hand, and positive and affective on the other.

\section{Different Underlying Pathophysiological Processes Associated with Different Intermediary Phenotypes May Account for a Substantial Part of Psychopathological Heterogeneity}

Findings at different levels of neuroscientific and neurocognitive research have suggested some evidence of meaningful contrasts in the underlying patterns of cerebral dysfunction in relation to the psychopathological heterogeneity of psychosis. Functional brain imaging studies have demonstrated different patterns of altered cerebral activity in schizophrenia. The negative (Andreasen, Paradiso, \& O'Leary, 1998; Sanfilipo et al., 2000; Schroder et al., 1996) and disorganization (Crider, 1997; Liddle et al., 1992) symptoms were associated with alterations in frontal lobe functioning, whereas auditory hallucinations produced normal activation of the left prefrontal cortex, but exhibited less activation of the left middle temporal gyrus and supplementary motor area (Lennox, Park, Medley, Morris, \& Jones, 2000; McGuire et al., 1995; Shergill, Brammer, Williams, Murray, \& McGuire, 2000; Shergill, Bullmore, Simmons, Murray, \& McGuire, 2000). At the level of neurotransmission, different patterns involving several interconnected limbic, cortical and subcortical structures have been implicated in the different symptom dimensions in psychosis. An overactivity in neurotransmission from DA cell bodies (DA-D2 receptors), located in the ventral tegmental area of the midbrain, may result in the development of positive symptoms. A hypodopaminergic state in the prefrontal cortical terminal fields of the mesocortical DA neurons (DA-D1 receptors) has been hypothesized to underlie cognitive impairment and negative symptoms of schizophrenia, and may in turn contribute to 
the disinhibition of subcortical DA function (Duncan, Sheitman, \& Lieberman, 1999; Kellendonk et al., 2006).

The putative intermediary phenotype of an altered sensitivity to stress, associated with genetic risk of psychosis, was associated with increased levels of affective and positive psychotic symptoms following exposure to small stressors in the flow of daily life (Myin-Germeys \& van Os, 2007), whilst it correlated negatively with cognitive impairment in patients with schizophrenia (Myin Germeys, Krabbendam, Jolles, Delespaul, \& van Os, 2002). Furthermore, positive psychotic symptomatology has been associated with impaired social cognition, such as alterations in self-monitoring (Bentall, 1990; Johns et al., 2001), a probabilistic reasoning bias referred to as jumping-to-conclusions (Garety \& Freeman, 1999; Garety, Kuipers, Fowler, Freeman, \& Bebbington, 2001; van Dael et al., 2006), and mentalizing deficits (Versmissen et al., 2007). Where examined, research suggests that the association between altered social cognition and symptoms is weakest for the negative and disorganization dimensions (van Dael et al., 2006).

In sum, neuroimaging, neuropharmacological and neurocognitive findings concur showing weak but systematic patterns of associations with symptom dimensions. This finding may explain at least some of the psychopathological heterogeneity in non-affective psychosis and may be refined in future studies to the extent that they become nosologically useful.

\section{Integrating Affective Dysregulation and Reality Distortion from a Perspective of Risk}

Psychopathological studies have shown psychotic experiences to co-occur in the range of affective disorders (Dunayevich \& Keck, 2000; Sareen, Cox, Goodwin, \& $\mathrm{G}, 2005$ ) and, conversely, high rates of affective symptoms have been demonstrated in patients diagnosed with DSM-IV non-affective psychotic disorders (Peralta \& Cuesta, 2009) and schizotypy (Debbane, van der Linden, Gex-Fabry, \& Eliez, 2009). Family studies demonstrate a degree of overlap in genetic liability between non-affective psychotic disorder and bipolar disorder (Cardno, Rijsdijk, Sham, Murray, \& McGuffin, 2002; Craddock \& Owen, 2007; Lichtenstein et al., 2009). Turning to epidemiological settings, subthreshold depressive and/or hypomanic symptoms have been shown to represent the behavioural expression of risk for more severe psychotic states such as bipolar disorder (Regeer et al., 2006; Tijssen et al., 2009). Furthermore, earlier general population surveys in Greece and the Netherlands have shown a high degree of overlap between psychotic experiences and affective symptoms below the threshold for clinical disorder (Kaymaz et al., 2007; Krabbendam, Myin-Germeys, Bak, \& van Os, 2005; Krabbendam et al., 2004; Krabbendam, Myin-Germeys, Hanssen et al., 2005; Stefanis et al., 2002). 
Nonetheless, the majority of general cohort studies on transition rates from subclinical symptoms to clinical syndromes did not examine co-expression of reality distortion and affective dysregulation. Chapter 4 connects reality distortion and affective dysregulation from a perspective of risk to the onset of clinical psychosis at a population-based level. The results suggest that psychotic experiences in the general population are non-specifically associated with affective dysregulation, in a dose-response fashion. In addition, persistence of psychotic experiences and the onset of impairment occur more often if there is a context of affective dysregulation - that is, the higher the affect dysregulation in terms of subclinical depressive or hypomanic symptoms, the higher the incidence and persistence of psychotic experiences, and the higher the incidence of clinical psychosis. In light of the accumulating evidence on overlap in genetic liability, suggesting a broad underlying vulnerability that expresses across the different psychosis categories, our findings of associations between affective dysregulation and psychotic experiences in the general population may thus in part reflect passive clustering of the behavioral expression of overlapping distributed genetic vulnerabilities.

The fact that co-expression of affective dysregulation predicted persistence of reality distortion and secondary impairment (chapter 4) suggests that, in addition to correlated genetic liabilities, a direct impact of affective regulation on clinical relevance of psychotic experiences may be hypothesized. Existent psychological models of symptom formation suggest that the emotional context, and associated beliefs or appraisals, may induce bias in logical reasoning processes and therefore provoke reality distortion in healthy individuals (Garety et al., 2001; Myin-Germeys $\&$ van Os, 2007). In the psychological model described by Garety and colleagues (2007), the experience of a stressful event is thought to potentially give rise to altered pre-existing beliefs and ongoing appraisals of experiences, due to a certain emotional change or a cognitive processing bias. These distorted processes may lead to aberrant experiences that may seem personally significant to the individual and are likely to trigger a search for an explanation as to their meaning and cause that is consistent with affect-associated beliefs. Biased appraisal processes may contribute to a judgment that the experience is in fact externally caused. It is the interpretation that causes the associated distress and disability, rather than the experience itself. Thus, maladaptive appraisal patterns, induced by emotional processes, are suggested to increase the risk for positive symptom formation in vulnerable individuals (Garety, Bebbington, Fowler, Freeman, \& Kuipers, 2007). Our findings provide support for a cognitive model of symptom formation, by demonstrating involvement of affective processes in the onset and persistence of reality distortion outside the context of disorder. 
Attempts have been made to integrate cognitive and neurobiological theories on psychosis (Garety et al., 2007; Kapur, 2003; van Os \& Kapur, 2009). There is evidence that psychotic symptoms are associated with underlying alterations in the dopamine system, including increased dopamine synaptic availability (Hirvonen et al., 2005), increased striatal dopamine synthesis (Huttunen et al., 2008; MeyerLindenberg et al., 2002) and increased dopamine reactivity to stress (Brunelin et al., 2008; Myin-Germeys \& van Os, 2007). Under normal circumstances, it is the context-driven activity of the dopamine system that mediates the experience of novelty and the acquisition of appropriate motivational salience, detecting new rewards in the environment that facilitate learning and goal-directed behavior. Certain cerebral vulnerabilities, occasioned by interplay between genetic and environmental risks, could trigger context-independent or context-inappropriate release of dopamine. Such a dysregulated dopamine system may cause aberrant assignment of salience and motivational significance to external objects (leading to delusions) and internal representations of percepts and memories (leading to hallucinations (Kapur, Mizrahi, \& Li, 2005).Thus, theory derived from existent psychological models of psychotic symptom formation predicts that affective dysregulation may impact directly on risk for reality distortion; additionally, the risk for clinically relevant psychosis may be particularly high in those who have a genetically liability for dopaminergic dysregulation facilitating aberrant salience attribution.

\section{Negative and Disorganized Features Increasing Risk for Positive Symptoms Increasing Risk for Impairment}

Several elements in the analysis of chapter 5 support the epidemiological prediction of the hypothesis that cognitive alterations and negative features associated with schizophrenia may represent alterations in brain development associated with distributed genetic risk, influencing a final common pathway of neurotransmitter dysregulation resulting in the onset of positive psychotic symptoms, particularly when combined with environmental exposures, such as cannabis, developmental trauma and urbanicity (van Os \& Kapur, 2009).

First, a pattern of socio-demographic associations for the negative-disorganized cluster with young age, male sex, single marital status and lower educational level was found, reflecting a profile indicative of developmental impairment which resembles the pattern seen in clinical samples (Leung \& Chue, 2000; Schultz et al., 1997). This developmental profile is in accordance with the association found in chapter 2 between neurocognitive impairment and the psychotic negative disorganized dimensions which are know to result in poorer social functioning (Macdonald, Hayes, \& Baglioni, 2000), poorer response to antipsychotic treatment 
(Erhart, Marder, \& Carpenter, 2006) and a worse prognosis (McGlashan \& Fenton, 1992).

Second, the socio-demographic associations of the positive dimension are in agreement with previous reports linking positive psychotic experiences to environmental risk factors such as cannabis exposure, urbanicity, self-reported trauma and lower educational level (Henquet et al., 2005; Spauwen, Krabbendam, Lieb, Wittchen, \& van Os, 2006a, 2006b; van Os et al., 2000). The link between these environmental risk factors and psychotic experiences has been described in terms of stress-dopamine (DA) interactions and sensitization (Collip et al., 2008). Linking cortical and subcortical dopaminergic processes, there is evidence that mesocortical DAergic innervation of the prefrontal cortex may regulate the activity of mesolimbic subcortical DA innervations and that the impact of environmental risk factors may result in taking the prefrontal cortical (PFC) "off-line", resulting in altered responsiveness of subcortical DAergic innervations (Deutch, Clark, \& Roth, 1990). Thus, when DA transmission is increased by exposure to stress or agonist drugs, there may be a shift in the balance of the system away from PFC control and toward limbic predominance, facilitating a DA hyperactivity in the ventral tegmental area of the midbrain, resulting in the development of positive symptoms (Laruelle et al., 2003).

Third, in the EDSP study, experiences indicative of the negative/disorganized and positive symptoms in the general population clustered together more often than predicted by chance, independent of depression. Thus, psychotic disorders such as schizophrenia are not the result of the chance cross-section of the separate liabilities of developmental impairment on the one hand and reality distortion on the other; they are linked together in a way that suggests fundamental overlap or interaction of underlying mechanisms.

Fourthly, the longitudinal analysis suggested that in this community sample negative-disorganized features preceded positive features rather than the other way round. Persistent expression of the negative-disorganized features predicted onset of psychotic experiences; in contrast, persistent expression of psychotic experiences did not predict onset of the negative-disorganized ones. This, together with the associations between positive features and environmental risks, suggests that the negative-disorganized symptoms cluster reflects an underlying vulnerability that, when combined with environmental exposures, results in positive symptoms increasing the risk of need for care. This latter suggestion is supported by the fact that the "comorbid" expression of positive and negative features increased the risk for impairment and, by implication, onset of clinical psychosis. 


\section{A Dynamic Model of the Onset of Clinical Psychosis from an Epidemiological Perspective}

The aforementioned population-based studies details the dynamics of the onset of clinically relevant psychosis (Figure 1) in a large sample of 3021 adolescents and young adults from the general population (EDSP study). Self-reported psychopathological experiences resembling the positive, negative-disorganized, depressive and hypomanic symptom clusters are shown to be expressed in attenuated form in otherwise "healthy" individuals (chapters 3, 4 and 5). Their population distribution suggests that they represent quantitative variations of normal human mentation. Based on the present studies, psychometric manifestations of the psychosis phenotype represent the behavioural expression of differential underlying liabilities to psychosis that, in interaction with environmental factors, contribute to the onset of clinical psychosis.

With regard to the positive psychotic dimension, evidence for continuity over time from subclinical persistent psychotic experiences to clinical psychotic states was tested using the longitudinal prospective design of the EDSP study (chapter 3; (M.D.G. Dominguez et al., 2009)). The dynamics preceding the clinical onset of psychotic disorder from a population perspective may be summarised descriptively as a Psychosis Proneness-Persistence-Impairment model of psychotic disorder, mechanisms of which were explained by behavioural, environmental and neurobiological interactions. A significant proportion of psychotic disorder may be conceptualised as the rare poor outcome of a common developmental phenotype. Positive psychotic experiences can thus be regarded as the behavioural expression of underlying distributed liability for psychosis.

With the processes underlying the various dimensions of psychotic psychopathology may be derived from the associations between psychosis dimensions and the intermediary phenotype of neurocognition in non-affective psychosis obtained from our meta-analytic work (chapter 2; (M. D. G. Dominguez et al., 2009)). Differential associations for the cluster of disorganized and negative symptoms on the one hand, and that of positive and affective on the other were in accordance with the formulation of the consilience model of psychosis (Van Os \& Kapur, 2009): the phenomenological expression of the altered brain development in the form of interpersonal style and negative features was associated with the intermediary phenotype of neurocognitive impairment, which may represent the pole of 'Development Expression' within the psychosis syndrome. In contrast, the opposite pole of 'Affective Dysregulation' and psychotic experiences was not associated with that of neurocognition. 
The epidemiological prediction of this model was tested in the EDSP study by examining:

(i) Associations between affective dysregulation and reality distortion in the general population resulted in higher risk for clinical psychosis in part reflecting overlapping distributed genetic vulnerabilities. The interaction between the two psychopathological domains was interpreted through the role of neurobiological activity and cognitive processes facilitated by the mechanism of aberrant salience attribution (chapter 4 ).

(ii) The association of negative and disorganized features corroborated that these psychopathological expressions, in co-existence with neurocognitive impairment, may represent alterations in brain development associated with distributed developmental genetic risk. When combined with environmental exposures, such as cannabis, developmental trauma and urbanicity, a final common pathway of neurotransmitter dysregulation may result in the onset of positive psychotic symptoms, increasing the risk for onset of clinical psychosis (chapter 5).

The present epidemiological work may contribute to tracing a dynamic model for the development of psychosis expression from mental health to pathological states; quantitative variations of normal human mentation in the context of interactions between individuals and their environments act in such a way that their interacting underlying processes do pose a risk for the onset of clinical psychosis.

Figure 1. A Dynamic Model of the Onset of Clinical Psychosis from an Epidemiological Perspective

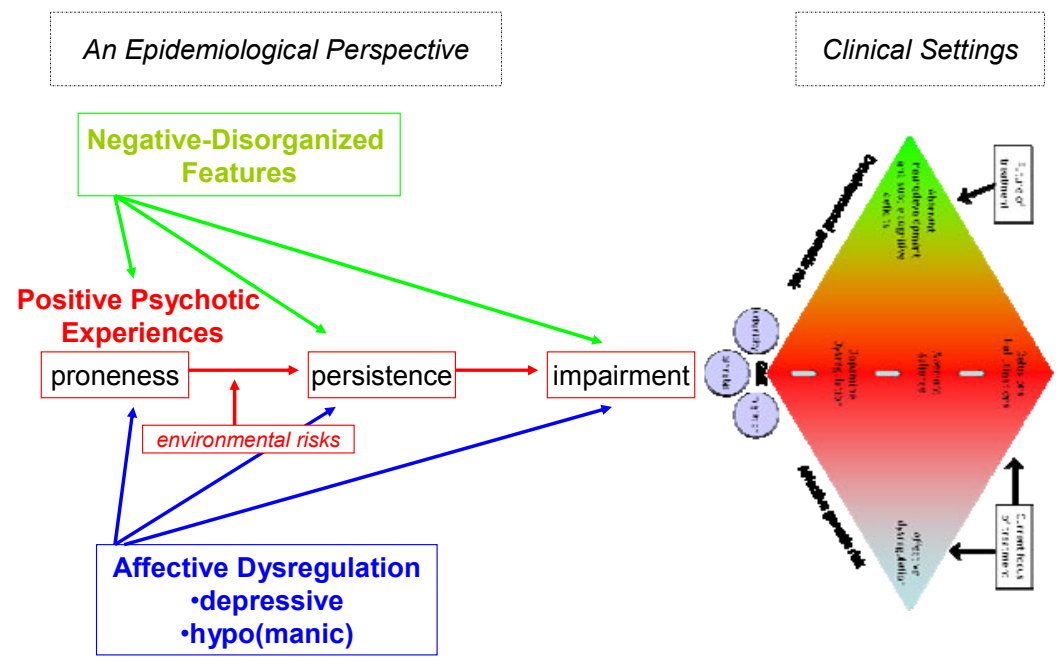




\section{Nosological Implications}

The findings of the present thesis raise questions about our conceptualization and nosological classification of psychotic disorders, with obvious repercussions to clinical practice in the diagnostic process, clinical management and intervention.

Given that there is quantitative variation in symptom dimension scores across current diagnostic categories rather than qualitative differences, it is unlikely that the current psychotic categories represent discrete nosological entities (Dikeos et al., 2006). Additionally, evidence has been given for shared genetic causes underlying diagnoses of psychotic disorders (Kendler \& Diehl, 1993), including bipolar disorder (Craddock \& Owen, 2007). Therefore, a flexible scoring of dimensions across all psychotic and major affective disorders may be potentially more informative than a system where categorical diagnoses are kept artificially dimension-specific. Models using both categorical and dimensional representations have shown better discriminative validity than either model independently, suggesting that the most powerful approach to classification is the complementary use of both categorical diagnoses and dimensional scores (Allardyce, McCreadie, Morrison, \& van Os, 2007). In this context, a major likely change in DSM-V and ICD-11 is expected to be the addition of dimensional indicators that can be applied across diagnostic categories of affective and non-affective psychotic disorder (van Os \& Kapur, 2009).

Our findings add evidence for continuity with normal mentation enlightening differential pathways of interaction across symptoms domains to the onset of clinical psychosis. Firstly, this shows that the underlying latent structure of psychopathology is continuous. Secondly, these differential pathways can be traced longitudinally: (i) the one characterized by affective dysregulation, closely linked to stress-related environmental factors, and (ii) that of developmental expression, portrayed by negative and disorganized features associated with cognitive alterations, which strongly resembles the epidemiological prediction of the purest clinical concept of non-affective psychosis.

\section{Clinical Implications}

Recent work suggests that it is possible to intervene early in the course of prodromal psychotic states in selected samples at the level of mental health services (Cannon et al., 2008; McGorry, 2002). Systematic reviews suggest that longer duration of untreated psychosis before treatment is initiated predicts poorer outcome (Farooq, Large, Nielssen, \& Waheed, 2009; Marshall et al., 2005; Perkins, $\mathrm{Gu}$, Boteva, \& Lieberman, 2005). At the level of the population, however, there is very little knowledge about how such prodromal states develop and which factors 
are important in the course of the very earliest expressions of psychosis. Given the possibilities of early intervention, more knowledge is urgently required on the population distribution of psychometric risk states for psychosis, and how these may develop into prodromes and clinical psychotic states (McGorry, Yung, Bechdolf, \& Amminger, 2008).

The results from our work can have important impact on early intervention. Our findings suggest that many clinical psychotic states can be traced to earlier attenuate phenotypic expressions years before the onset of the illness. The findings therefore support the notion of early intervention in psychosis although not all onsets of clinical psychotic states may benefit from these. In addition, the findings suggest that isolated subclinical positive psychotic experiences themselves cannot be considered as "prodromal" symptoms in spite of their severity; however, psychometric expression of psychosis risk may be particularly informative if assessed (i) repeatedly, (ii) in interaction with psychopathological dimensions rather than isolated psychotic symptoms and (iii) in the context of environmental risks (Cougnard et al., 2007). Multiple assessments will also help reduce the risk of false positive findings (McGorry et al., 1995; Van Os \& Delespaul, 2005).

\section{Directions for Future Research}

The present thesis offers a longitudinal epidemiological approach of the dynamic process to the onset of clinical psychosis, based on psychopathological, functional, socio-demographic and environmental data. Its conceptual implications question our understanding of psychosis; the repercussions of which need to be tested within the recent advances of genetics, imaging, cognitive sciences and psychopharmacology.

In the present work, the associations between psychosis dimensions and the intermediary phenotype of neurocognition in clinical psychosis were investigated (chapter 2). Establishing differential patterns of the intermediary phenotypes in the field of cognitive sciences, combining neurocognition (M. D. G. Dominguez et al., 2009) and the emerging field of social cognition (Garety et al., 2007), among the different psychopathological dimensions of clinical psychosis, and investigating their differential functional outcomes (Ventura, Hellemann, Thames, Koellner, \& Nuechterlein, 2009), may yield further insight in the underlying mechanisms of psychosis heterogeneity.

Turning to the population-based research, psychosis epidemiology can provide information to guide future research. However, while epidemiology is useful for generating candidate risk factors and tracing the longitudinal course of phenomenological features, it can not always deliver studies that enlighten 
pathophysiology and prove causality (McGrath \& Richards, 2009). Genetic epidemiological studies suggest that gene-environment interaction in schizophrenia and related diagnostic categories is common (van Os, Rutten, \& Poulton, 2008) and recent studies are suggestive for interaction between environmental exposures and specific molecular genetic variation (Caspi et al., 2005; Henquet et al., 2006). Likewise, imaging studies combining structural brain volume measures with functional activity enlighten the mechanisms how neurochemical abnormalities may converge to cause psychosis (Howes \& Kapur, 2009). Therefore, the challenge in the years to come is to bring together the various disciplines that are necessary to examine models of psychosis pathophysiology and disease causation.

\section{References}

Akiskal, H. S., \& Vazquez, G. H. (2006). [Widening the borders of the bipolar disorder: validation of the concept of bipolar spectrum]. Vertex, 17(69), 340-346.

Aleman, A., Hijman, R., de Haan, E. H., \& Kahn, R. S. (1999). Memory impairment in schizophrenia: A meta-analysis. American Journal of Psychiatry, 156(9), 1358-1366.

Allardyce, J., McCreadie, R. G., Morrison, G., \& van Os, J. (2007). Do symptom dimensions or categorical diagnoses best discriminate between known risk factors for psychosis? Soc Psychiatry Psychiatr Epidemiol, 42(6), 429-437.

Allardyce, J., Suppes, T., \& Van Os, J. (2007). Dimensions and the psychosis phenotype. Int J Methods Psychiatr Res, 16 Suppl 1 2007, S34-40.

Andreasen, N. C., Paradiso, S., \& O’Leary, D. S. (1998). "Cognitive dysmetria" as an integrative theory of schizophrenia: A dysfunction in cortical-subcortical-cerebellar circuitry? Schizophrenia Bulletin, 24(2), 203-218.

Angst, J., \& Cassano, G. (2005). The mood spectrum: improving the diagnosis of bipolar disorder. Bipolar Disord, 7 Suppl 4, 4-12.

Artaloytia, J. F., Arango, C., Lahti, A., Sanz, J., Pascual, A., Cubero, P., et al. (2006). Negative signs and symptoms secondary to antipsychotics: a double-blind, randomized trial of a single dose of placebo, haloperidol, and risperidone in healthy volunteers. Am J Psychiatry, 163(3), 488-493.

Brunelin, J., d'Amato, T., van Os, J., Cochet, A., Suaud-Chagny, M. F., \& Saoud, M. (2008). Effects of acute metabolic stress on the dopaminergic and pituitary-adrenal axis activity in patients with schizophrenia, their unaffected siblings and controls. Schizophr Res, 100(1-3), 206-211.

Cannon, T. D., Cadenhead, K., Cornblatt, B., Woods, S. W., Addington, J., Walker, E., et al. (2008). Prediction of psychosis in youth at high clinical risk: a multisite longitudinal study in North America. Arch Gen Psychiatry, 65(1), 28-37.

Cannon, T. D., Cornblatt, B., \& McGorry, P. (2007). The empirical status of the ultra high-risk (prodromal) research paradigm. Schizophr Bull, 33(3), 661-664.

Cardno, A. G., Rijsdijk, F. V., Sham, P. C., Murray, R. M., \& McGuffin, P. (2002). A twin study of genetic relationships between psychotic symptoms. Am J Psychiatry, 159(4), 539-545.

Carpenter, W. T., Jr., Heinrichs, D. W., \& Alphs, L. D. (1985). Treatment of negative symptoms. Schizophr Bull, 11(3), 440-452.

Caspi, A., Moffitt, T. E., Cannon, M., McClay, J., Murray, R., Harrington, H., et al. (2005). Moderation of the effect of adolescent-onset cannabis use on adult psychosis by a functional polymorphism in the catecholO-methyltransferase gene: longitudinal evidence of a gene X environment interaction. Biol Psychiatry, 57(10), 1117-1127.

Chapman, L. J., Chapman, J. P., Kwapil, T. R., Eckblad, M., \& Zinser, M. C. (1994). Putatively psychosisprone subjects 10 years later. J Abnorm Psychol, 103(2), 171-183.

Collip, D., Myin-Germeys, I., \& Van Os, J. (2008). Does the concept of "sensitization" provide a plausible mechanism for the putative link between the environment and schizophrenia? Schizophr Bull, 34(2), 220-225. 
Cougnard, A., Marcelis, M., Myin-Germeys, I., R, D. E. G., Vollebergh, W., Krabbendam, L., et al. (2007). Does normal developmental expression of psychosis combine with environmental risk to cause persistence of psychosis? A psychosis proneness-persistence model. Psychol Med, 37(4), 513-527.

Cougnard, A., Salmi, L. R., Salamon, R., \& Verdoux, H. (2005). A decision analysis model to assess the feasibility of the early detection of psychosis in the general population. Schizophr Res, 74(1), 27-36.

Craddock, N., \& Owen, M. J. (2007). Rethinking psychosis: the disadvantages of a dichotomous classification now outweigh the advantages. World Psychiatry, 6(2), 84-91.

Crider, A. (1997). Perseveration in schizophrenia. Schizophrenia Bulletin, 23(1), 63-74.

Debbane, M., Van der Linden, M., Gex-Fabry, M., \& Eliez, S. (2009). Cognitive and emotional associations to positive schizotypy during adolescence. J Child Psychol Psychiatry, 50(3), 326-334.

Deutch, A. Y., Clark, W. A., \& Roth, R. H. (1990). Prefrontal cortical dopamine depletion enhances the responsiveness of mesolimbic dopamine neurons to stress. Brain Res, 521(1-2), 311-315.

Dikeos, D. G., Wickham, H., McDonald, C., Walshe, M., Sigmundsson, T., Bramon, E., et al. (2006). Distribution of symptom dimensions across Kraepelinian divisions. The British Journal of Psychiatry 189, 346-353.

Dominguez, M. D. G., Viechtbauer, W., Simons, C. J., van Os, J., \& Krabbendam, L. (2009). Are psychotic psychopathology and neurocognition orthogonal? A systematic review of their associations. Psychol Bull, 135(1), 157-171.

Dominguez, M. D. G., Wichers, M., Lieb, R., Wittchen, H. U., \& van Os, J. (2009). Evidence That Onset of Clinical Psychosis Is an Outcome of Progressively More Persistent Subclinical Psychotic Experiences: An 8-Year Cohort Study. Schizophrenia Bulletin.

Dunayevich, E., \& Keck, P. E., Jr. (2000). Prevalence and description of psychotic features in bipolar mania. Curr Psychiatry Rep, 2(4), 286-290.

Duncan, G. E., Sheitman, B. B., \& Lieberman, J. A. (1999). An integrated view of pathophysiological models of schizophrenia. Brain Research. Brain Research Reviews, 29(2-3), 250-264.

Erhart, S. M., Marder, S. R., \& Carpenter, W. T. (2006). Treatment of schizophrenia negative symptoms: future prospects. Schizophr Bull, 32(2), 234-237.

Escher, S., Romme, M., Buiks, A., Delespaul, P., \& Van Os, J. (2002). Independent course of childhood auditory hallucinations: a sequential 3-year follow-up study. Br J Psychiatry Suppl, 43, s10-18.

Farooq, S., Large, M., Nielssen, O., \& Waheed, W. (2009). The relationship between the duration of untreated psychosis and outcome in low-and-middle income countries: a systematic review and meta analysis. Schizophr Res, 109(1-3), 15-23.

Fioravanti, M., Carlone, O., Vitale, B., Cinti, M. E., \& Clare, L. (2005). A meta-analysis of cognitive deficits in adults with a diagnosis of schizophrenia. Neuropsychology Review, 15(2), 73-95.

Garety, P. A., Bebbington, P., Fowler, D., Freeman, D., \& Kuipers, E. (2007). Implications for neurobiological research of cognitive models of psychosis: a theoretical paper. Psychol Med, 37(10), 1377-1391.

Garety, P. A., Kuipers, E., Fowler, D., Freeman, D., \& Bebbington, P. E. (2001). A cognitive model of the positive symptoms of psychosis. Psychological Medicine, 31(2), 189-195.

Hanssen, M., Bak, M., Bijl, R., Vollebergh, W., \& van Os, J. (2005). The incidence and outcome of subclinical psychotic experiences in the general population. Br J Clin Psychol, 44(Pt 2), 181-191.

Heinrichs, R. W., \& Zakzanis, K. K. (1998). Neurocognitive deficit in schizophrenia: A quantitative review of the evidence. Neuropsychology, 12(3), 426-445.

Henquet, C., Krabbendam, L., Spauwen, J., Kaplan, C., Lieb, R., Wittchen, H. U., et al. (2005). Prospective cohort study of cannabis use, predisposition for psychosis, and psychotic symptoms in young people. Bmj, 330(7481), 11.

Henquet, C., Rosa, A., Krabbendam, L., Papiol, S., Fananas, L., Drukker, M., et al. (2006). An experimental study of catechol-o-methyltransferase Val158Met moderation of delta-9-tetrahydrocannabinol-induced effects on psychosis and cognition. Neuropsychopharmacology, 31(12), 2748-2757.

Hirvonen, J., van Erp, T. G., Huttunen, J., Aalto, S., Nagren, K., Huttunen, M., et al. (2005). Increased caudate dopamine D2 receptor availability as a genetic marker for schizophrenia. Arch Gen Psychiatry, 62(4), 371-378.

Howes, O. D., \& Kapur, S. (2009). The dopamine hypothesis of schizophrenia: version III--the final common pathway. Schizophr Bull, 35(3), 549-562. 
Huttunen, J., Heinimaa, M., Svirskis, T., Nyman, M., Kajander, J., Forsback, S., et al. (2008). Striatal dopamine synthesis in first-degree relatives of patients with schizophrenia. Biol Psychiatry, 63(1), 114117.

Johnson Selfridge, M., \& Zalewski, C. (2001). Moderator variables of executive functioning in schizophrenia: Meta-analytic findings. Schizophrenia Bulletin, 27(2), 305-316.

Kapur, S. (2003). Psychosis as a state of aberrant salience: a framework linking biology, phenomenology, and pharmacology in schizophrenia. Am J Psychiatry, 160(1), 13-23.

Kapur, S. (2004). How antipsychotics become anti-"psychotic"--from dopamine to salience to psychosis. Trends Pharmacol Sci, 25(8), 402-406.

Kapur, S., Mizrahi, R., \& Li, M. (2005). From dopamine to salience to psychosis--linking biology, pharmacology and phenomenology of psychosis. Schizophr Res, 79(1), 59-68.

Kaymaz, N., van Os, J., de Graaf, R., Ten Have, M., Nolen, W., \& Krabbendam, L. (2007). The impact of subclinical psychosis on the transition from subclinicial mania to bipolar disorder. $J$ Affect Disord, 98(12), 55-64.

Keefe, R. S., Bilder, R. M., Harvey, P. D., Davis, S. M., Palmer, B. W., Gold, J. M., et al. (2006). Baseline neurocognitive deficits in the CATIE schizophrenia trial. Neuropsychopharmacology, 31(9), 2033-2046.

Kellendonk, C., Simpson, E. H., Polan, H. J., Malleret, G., Vronskaya, S., Winiger, V., et al. (2006). Transient and selective overexpression of dopamine D2 receptors in the striatum causes persistent abnormalities in prefrontal cortex functioning. Neuron, 49(4), 603-615.

Kendler, K. S., \& Diehl, S. R. (1993). The genetics of schizophrenia: a current, genetic-epidemiologic perspective. Schizophr Bull, 19(2), 261-285.

Krabbendam, L., Myin-Germeys, I., Bak, M., \& van Os, J. (2005). Explaining transitions over the hypothesized psychosis continuum. Aust N Z J Psychiatry, 39(3), 180-186.

Krabbendam, L., Myin-Germeys, I., De Graaf, R., Vollebergh, W., Nolen, W. A., Iedema, J., et al. (2004). Dimensions of depression, mania and psychosis in the general population. Psychol Med, 34(7), 11771186.

Krabbendam, L., Myin-Germeys, I., Hanssen, M., de Graaf, R., Vollebergh, W., Bak, M., et al. (2005). Development of depressed mood predicts onset of psychotic disorder in individuals who report hallucinatory experiences. Br J Clin Psychol, 44(Pt 1), 113-125.

Laruelle, M. (2000). The role of endogenous sensitization in the pathophysiology of schizophrenia: implications from recent brain imaging studies. Brain Res Brain Res Rev, 31(2-3), 371-384.

Laruelle, M., Kegeles, L. S., \& Abi-Dargham, A. (2003). Glutamate, dopamine, and schizophrenia: from pathophysiology to treatment. Ann N Y Acad Sci, 1003, 138-158.

Lataster, T., Myin-Germeys, I., Derom, C., Thiery, E., \& van Os, J. (2009). Evidence that self-reported psychotic experiences represent the transitory developmental expression of genetic liability to psychosis in the general population. Am J Med Genet B Neuropsychiatr Genet.

Lee, J., \& Park, S. (2005). Working memory impairments in schizophrenia: A meta-analysis. Journal of Abnormal Psychology, 114(4), 599-611.

Lennox, B. R., Park, S. B., Medley, I., Morris, P. G., \& Jones, P. B. (2000). The functional anatomy of auditory hallucinations in schizophrenia. Psychiatry Research, 100(1), 13-20.

Leung, A., \& Chue, P. (2000). Sex differences in schizophrenia, a review of the literature. Acta Psychiatr Scand Suppl, 401, 3-38.

Lichtenstein, P., Yip, B. H., Bjork, C., Pawitan, Y., Cannon, T. D., Sullivan, P. F., et al. (2009). Common genetic determinants of schizophrenia and bipolar disorder in Swedish families: a population-based study. Lancet, 373(9659), 234-239.

Liddle, P. F., Friston, K. J., Frith, C. D., Hirsch, S. R., Jones, T., \& Frackowiak, R. S. (1992). Patterns of cerebral blood flow in schizophrenia. The British Journal of Psychiatry, 160, 179-186.

Lieb, R., Isensee, B., von Sydow, K., \& Wittchen, H. U. (2000). The Early Developmental Stages of Psychopathology Study (EDSP): a methodological update. Eur Addict Res, 6(4), 170-182.

Lieberman, J. A., Sheitman, B. B., \& Kinon, B. J. (1997). Neurochemical sensitization in the pathophysiology of schizophrenia: deficits and dysfunction in neuronal regulation and plasticity. Neuropsychopharmacology, 17(4), 205-229.

Macdonald, E. M., Hayes, R. L., \& Baglioni, A. J., Jr. (2000). The quantity and quality of the social networks of young people with early psychosis compared with closely matched controls. Schizophr Res, 46(1), 2530. 
Maric, N., Myin-Germeys, I., Delespaul, P., de Graaf, R., Vollebergh, W., \& Van Os, J. (2004). Is our concept of schizophrenia influenced by Berkson's bias? Soc Psychiatry Psychiatr Epidemiol, 39(8), 600-605.

Marshall, M., Lewis, S., Lockwood, A., Drake, R., Jones, P., \& Croudace, T. (2005). Association between duration of untreated psychosis and outcome in cohorts of first-episode patients: a systematic review. Arch Gen Psychiatry, 62(9), 975-983.

McGlashan, T. H., \& Fenton, W. S. (1992). The positive-negative distinction in schizophrenia. Review of natural history validators. Arch Gen Psychiatry, 49(1), 63-72.

McGorry, P. D. (2002). The recognition and optimal management of early psychosis: an evidence-based reform. World Psychiatry, 1(2), 76-83.

McGorry, P. D., McFarlane, C., Patton, G. C., Bell, R., Hibbert, M. E., Jackson, H. J., et al. (1995). The prevalence of prodromal features of schizophrenia in adolescence: a preliminary survey. Acta Psychiatr Scand, 92(4), 241-249.

McGorry, P. D., Yung, A. R., Bechdolf, A., \& Amminger, P. (2008). Back to the future: predicting and reshaping the course of psychotic disorder. Arch Gen Psychiatry, 65(1), 25-27.

McGrath, J. J., \& Richards, L. J. (2009). Why schizophrenia epidemiology needs neurobiology--and vice versa. Schizophr Bull, 35(3), 577-581.

McGuire, P. K., Silbersweig, D. A., Wright, I., Murray, R. M., David, A. S., Frackowiak, R. S., et al. (1995). Abnormal monitoring of inner speech: A physiological basis for auditory hallucinations. Lancet, 346(8975), 596-600.

Meyer-Lindenberg, A., Miletich, R. S., Kohn, P. D., Esposito, G., Carson, R. E., Quarantelli, M., et al. (2002). Reduced prefrontal activity predicts exaggerated striatal dopaminergic function in schizophrenia. Nat Neurosci, 5(3), 267-271.

Myin-Germeys, I., \& van Os, J. (2007). Stress-reactivity in psychosis: evidence for an affective pathway to psychosis. Clin Psychol Rev, 27(4), 409-424.

Myin Germeys, I., Krabbendam, L., Jolles, J., Delespaul, P. A., \& van Os, J. (2002). Are cognitive impairments associated with sensitivity to stress in schizophrenia? An experience sampling study. American Journal of Psychiatry, 159(3), 443-449.

Pelletier, M., Achim, A. M., Montoya, A., Lal, S., \& Lepage, M. (2005). Cognitive and clinical moderators of recognition memory in schizophrenia: A meta-analysis. Schizophrenia Research, 74(2-3), 233-252.

Peralta, V., \& Cuesta, M. J. (2009). Characterization of affective domains within the nonaffective psychotic disorders. Schizophr Res, 111(1-3), 61-69.

Perkins, D. O., Gu, H., Boteva, K., \& Lieberman, J. A. (2005). Relationship between duration of untreated psychosis and outcome in first-episode schizophrenia: a critical review and meta-analysis. Am J Psychiatry, 162(10), 1785-1804.

Peters, E. R., Joseph, S. A., \& Garety, P. A. (1999). Measurement of delusional ideation in the normal population: introducing the PDI (Peters et al. Delusions Inventory). Schizophr Bull, 25(3), 553-576.

Poulton, R., Caspi, A., Moffitt, T. E., Cannon, M., Murray, R., \& Harrington, H. (2000). Children's selfreported psychotic symptoms and adult schizophreniform disorder: a 15-year longitudinal study. Arch Gen Psychiatry, 57(11), 1053-1058.

Reed, V., Gander, F., \& Pfister, H. (1998). To what degree does the Composite International Diagnostic Interview (CID) correctly identify DSM-IV disorders? Testing validity issues in a clinical sample. . International Journal of Methods in Psychiatric Research, 7, 142-155.

Regeer, E. J., Krabbendam, L., de Graaf, R., ten Have, M., Nolen, W. A., \& van Os, J. (2006). A prospective study of the transition rates of subthreshold (hypo)mania and depression in the general population. Psychol Med, 36(5), 619-627.

Sanfilipo, M., Lafargue, T., Rusinek, H., Arena, L., Loneragan, C., Lautin, A., et al. (2000). Volumetric measure of the frontal and temporal lobe regions in schizophrenia: Relationship to negative symptoms. Archives of General Psychiatry, 57(5), 471-480.

Sareen, J., Cox, B. J., Goodwin, R. D., \& G, J. G. A. (2005). Co-occurrence of posttraumatic stress disorder with positive psychotic symptoms in a nationally representative sample. J Trauma Stress, 18(4), 313322.

Schroder, J., Buchsbaum, M. S., Siegel, B. V., Geider, F. J., Lohr, J., Tang, C., et al. (1996). Cerebral metabolic activity correlates of subsyndromes in chronic schizophrenia. Schizophrenia Research, 19(1), 41-53. 
Schultz, S. K., Miller, D. D., Oliver, S. E., Arndt, S., Flaum, M., \& Andreasen, N. C. (1997). The life course of schizophrenia: Age and symptom dimensions. Schizophrenia Research, 23(1), 15-23.

Shergill, S. S., Brammer, M. J., Williams, S. C., Murray, R. M., \& McGuire, P. K. (2000). Mapping auditory hallucinations in schizophrenia using functional magnetic resonance imaging. Archives of General Psychiatry, 57(11), 1033-1038.

Shergill, S. S., Bullmore, E., Simmons, A., Murray, R., \& McGuire, P. (2000). Functional anatomy of auditory verbal imagery in schizophrenic patients with auditory hallucinations. American Journal of Psychiatry, 157(10), 1691-1693.

Spauwen, J., Krabbendam, L., Lieb, R., Wittchen, H. U., \& van Os, J. (2006a). Evidence that the outcome of developmental expression of psychosis is worse for adolescents growing up in an urban environment. Psychol Med, 36(3), 407-415.

Spauwen, J., Krabbendam, L., Lieb, R., Wittchen, H. U., \& van Os, J. (2006b). Impact of psychological trauma on the development of psychotic symptoms: relationship with psychosis proneness. $\mathrm{Br} J$ Psychiatry, 188, 527-533.

Stefanis, N. C., Hanssen, M., Smirnis, N. K., Avramopoulos, D. A., Evdokimidis, I. K., Stefanis, C. N., et al. (2002). Evidence that three dimensions of psychosis have a distribution in the general population. Psychol Med, 32(2), 347-358.

Tijssen, M. J., van Os, J., Wittchen, H. U., Lieb, R., Beesdo, K., Mengelers, R., et al. (2009). Evidence that bipolar disorder is the poor outcome fraction of a common developmental phenotype: an 8-year cohort study in young people. Psychol Med, 1-11.

van Os, J., \& Delespaul, P. (2005). Toward a world consensus on prevention of schizophrenia. Dialogues Clin Neurosci, 7(1), 53-67.

van Os, J., Hanssen, M., Bijl, R. V., \& Ravelli, A. (2000). Strauss (1969) revisited: a psychosis continuum in the general population? Schizophr Res, 45(1-2), 11-20.

van Os, J., \& Kapur, S. (2009). Schizophrenia. Lancet, 374(9690), 635-645.

van Os, J., Linscott, R. J., Myin-Germeys, I., Delespaul, P., \& Krabbendam, L. (2009). A systematic review and meta-analysis of the psychosis continuum: evidence for a psychosis proneness-persistenceimpairment model of psychotic disorder. Psychol Med, 39(2), 179-195.

van Os, J., Rutten, B. P., \& Poulton, R. (2008). Gene-environment interactions in schizophrenia: review of epidemiological findings and future directions. Schizophr Bull, 34(6), 1066-1082.

van Os, J., Verdoux, H., Maurice-Tison, S., Gay, B., Liraud, F., Salamon, R., et al. (1999). Self-reported psychosis-like symptoms and the continuum of psychosis. Soc Psychiatry Psychiatr Epidemiol, 34(9), 459-463.

Ventura, J., Hellemann, G. S., Thames, A. D., Koellner, V., \& Nuechterlein, K. H. (2009). Symptoms as mediators of the relationship between neurocognition and functional outcome in schizophrenia: A metaanalysis. Schizophr Res.

Verdoux, H., van Os, J., Maurice-Tison, S., Gay, B., Salamon, R., \& Bourgeois, M. (1998). Is early adulthood a critical developmental stage for psychosis proneness? A survey of delusional ideation in normal subjects. Schizophr Res, 29(3), 247-254.

Welham, J., Scott, J., Williams, G., Najman, J., Bor, W., O’Callaghan, M., et al. (2009). Emotional and behavioural antecedents of young adults who screen positive for non-affective psychosis: a 21-year birth cohort study. Psychol Med, 39(4), 625-634.

Werbeloff, N., Drukker, M., \& Dohrenwend, B. (2009). Self-Reported Psychotic Symptoms in the Community are Associated with Increased Risk of Later Hospitalization for Non-Affective Psychotic Disorders. In C. Abstract (Ed.) (Vol. 35, pp. 74): Schizophrenia Bulletin.

Wittchen, H. U., Perkonigg, A., Lachner, G., \& Nelson, C. B. (1998). Early developmental stages of psychopathology study (EDSP): objectives and design. Eur Addict Res, 4(1-2), 18-27.

Wolf, M. E., White, F. J., Nassar, R., Brooderson, R. J., \& Khansa, M. R. (1993). Differential development of autoreceptor subsensitivity and enhanced dopamine release during amphetamine sensitization. $J$ Pharmacol Exp Ther, 264(1), 249-255. 



\section{SUMMARY}

This thesis, A Dynamic Model of the Onset of Clinical Psychosis from an Epidemiological Perspective, examines the psychosis phenotype as it exists in nature and the dynamic process of psychosis evolution from mental wellbeing to the onset of clinical psychosis, from an epidemiological perspective.

Chapter 1 describes epidemiological methods examining person-environment interactions and how they influence the development of major psychiatric disorders. It focuses on the occurrence of the psychosis phenotype in nature and the potentially predictive value of conceptualizing the evolution of psychosis as a dynamic process. Psychosis is firstly considered within the clinical setting, where psychosis was initially described, investigated and conceptualized. Clinical heterogeneity is not fully explained by the categorical diagnoses found in the current classification systems. One way to more fully define the phenomenological variation is to search for patterns of overlap across symptom dimensions. A narrative review of inter-dimensional correlations is presented, as well as associations between psychosis dimensions and the intermediary phenotype of neurocognition. In this context, a consilience model of clinical psychosis, which integrates cognitive and neurobiological theories of psychosis and, in particular, establishes links among phenomenological heterogeneity, intermediary phenotypes and pathophysiological processes, is presented.

Secondly, the population-based studies of psychotic-like experiences and the continuity between clinical and subclinical psychosis phenotypes are presented together with their conceptual implications for the consilience model of psychosis. The crucial issues of psychometric assessment with self-report instruments and that of clinical relevance are discussed in order to introduce the aims of the present thesis: (i) to assess how universal psychotic symptoms are and to introduce the possibility that they represent quantitative variations of normal human mentation, and (ii) to test the epidemiological prediction of a dynamic model to the onset of clinical psychosis. For that purpose, the prospective, observational Early Developmental Stages of Psychopathology (EDSP) study consisting of a large, general population sample of 3021 adolescents and young adults from Munich, Germany, followed over a period of up to ten years is presented. 
Chapter 2 investigates the association between psychotic psychopathology and the intermediary phenotype of neurocognition in non-affective psychosis since demonstrating differential patterns of cognitive performance among the different psychopathological dimensions could yield insight into the underlying mechanisms which result in the ubiquitous heterogeneity inherent in the psychosis phenotype. A systematic review of published studies examining the correlations between symptom dimensions and neuropsychological performance was performed- the abstracts of 3129 articles were examined for possible inclusion in the analysis. Our meta-analysis (58 studies, 5009 individuals) showed negative and disorganized dimensions to be significantly but modestly associated with cognitive deficits, whereas affective and positive dimensions were not. The patterns of association for the four psychosis dimensions were stable across neurocognitive domains and independent of age, gender and chronicity of illness. We therefore concluded that psychotic symptoms and neurocognition are not entirely orthogonal, suggesting that differential cerebral mechanisms may underlie the disorganized and negative symptoms dimensions versus positive and affective ones.

Chapter 3 describes data from the large EDSP sample of adolescents and young adults in the general population. To our knowledge, this is the first study examining the influence of persistence of subclinical psychotic experiences in relation to the transition to clinically relevant psychosis. The hypothesis was investigated that developmental expression of psychometric risk, in the form of subclinical psychotic experiences in the general population, is usually transitory but in some instances may become abnormally persistent and progress to a clinical psychotic state. The findings are i), subclinical psychosis was confirmed to be common and mostly transitory, ii) persistent subclinical positive psychotic-like symptoms are a marker of emerging clinical relevant psychopathology. A doseresponse gradient was demonstrated: the longer the duration of subclinical psychosis, the greater the risk of developing clinical impairment, iii) although most subclinical psychosis was transitory, relationship between the clinical and the subclinical manifestations was not negligible: a significant proportion of psychotic disorders were best conceptualised as the poor outcome of a relatively common developmental phenotype characterised by persistence of psychometrically detectable subclinical psychotic experiences. For some individuals, psychosis is thus the result of a long developmental process of subclinical experiences and functional deficits. This may be summarised descriptively as a psychosis pronenesspersistence-impairment model of psychotic disorder. Possible mechanisms explaining the transition from persistence of subclinical psychosis to impairment are likely based on behavioural, environmental and neurobiological interactions.

Chapter 4 describes data from the EDSP study connecting reality distortion and affective dysregulation from a risk perspective, at a population-based level. It 
examines the relationship between attenuated positive and affective symptoms and the onset of clinical psychosis. The results suggest that psychotic experiences in the general population are associated with affective dysregulation, and provide evidence of a dose response gradient. Furthermore, persistence of psychotic-like experiences and the onset of impairment are higher when affective dysregulation is present. In light of the accumulating evidence suggesting an overlap in genetic liability, across the different psychosis categories, our findings suggest genetic liabilities underlying affective and non-affective psychotic syndromes may be expressed as correlated dimensions in the general population. The longitudinal prediction of reality distortion in affective dysregulation is discussed in the context of existent psychological models of symptom formation and neurobiological activity, suggesting a process of misassignment of emotional salience underlying this connection.

Chapter 5 describes data from the EDSP study examining the natural occurrence of negative symptoms in the general population and if and how attenuated positive and negative measures of psychopathology, from a risk perspective, may contribute to the onset of clinical psychosis. A priori hypotheses suggest that cognitive/negative symptoms associated with schizophrenia may represent alterations in brain development associated with distributed genetic risk. When combined with stress-related environmental exposures, a final common pathway of neurotransmitter dysregulation may result in the onset of positive psychotic symptoms. This study highlighted the natural occurrence of attenuated negative symptoms in otherwise healthy individuals, independent of antipsychotic treatment. Negative symptoms revealed a pattern of socio-demographic associations indicative of developmental impairment, whereas the positive symptoms were associated with environmental risk factors such as trauma, cannabis use and urbanicity. Both psychosis dimensions were frequent and occurred in combination more often than predicted by chance. Negative symptoms predicted positive symptoms over time, and co-occurrence of positive and negative symptoms was predictive of clinical relevance in terms of secondary functional impairment and help-seeking behaviour. Results suggested that the liabilities underlying negative features of psychotic illness are distributed at a population level and drive the ontogenesis of positive psychotic experiences after exposure to environmental risks, increasing the likelihood of impairment and need for care.

In Chapter 6, the results of this thesis including the systematic review of clinical studies and the population-based studies are discussed and integrated into $A$ Dynamic Model of the Onset of Clinical Psychosis from an Epidemiological Perspective. Nosological and clinical implications of the findings of this thesis are provided as well as directions for future research. 



\section{SAMENVATTING}

Deze thesis, Een dynamisch model van het begin van klinische psychose vanuit een epidemiologisch perspectief, onderzoekt het psychosefenotype zoals het bestaat in de natuur en het dynamische proces van psychose-evolutie van mentaal welzijn naar het begin van klinische psychose, vanuit een epidemiologisch perspectief.

Hoofdstuk 1 beschrijft epidemiologische methodes die interacties onderzoeken tussen persoon en omgeving en de invloed daarvan op het ontwikkelen van belangrijke psychiatrische stoornissen. Het hoofdstuk focust op het voorkomen van het psychosefenotype in de natuur en de potentiële voorspellende waarde voor het conceptualiseren van de evolutie van een psychose als een dynamisch proces.

Psychose wordt in de eerste plaats gezien binnen het klinische kader, waarin men psychose voor het eerst beschreef, onderzocht en conceptualiseerde. Klinische heterogeniteit wordt niet volledig verklaard door de categorische diagnoses in de huidige classificatiesystemen. Een manier om de fenomenologische variatie volledig te definiëren is om te zoeken naar overlappingspatronen in de symptoomdimensies. Een narratief overzicht van inter-dimensionale correlaties wordt voorgesteld, alsook verbanden tussen psychosedimensies en het intermediaire fenotype van neurocognitie. In deze context wordt een verzoeningsmodel van klinische psychose voorgesteld dat cognitieve en neurobiologische theorieën over psychose integreert en, in het bijzonder, verbanden legt tussen fenomenologische heterogeniteit, intermediaire fenotypen en pathofysiologische processen.

In de tweede plaats worden de op populatie gebaseerde studies van op psychose gelijkende ervaringen en de continuiteit tussen klinische en subklinische psychosefenotypen voorgesteld samen met hun conceptuele implicaties voor het verzoeningsmodel van psychose. De cruciale kwesties van psychometrische beoordeling met tools voor zelfrapportage en van klinische relevantie worden besproken ter inleiding van de doelstellingen van deze thesis: (i) beoordelen hoe universeel psychotische symptomen zijn en het suggereren dat ze kwantitatieve variaties vertonen van de normale menselijke geestestoestand, en (ii) het testen van de epidemiologische voorspelling van een dynamisch model tot het begin van klinische psychose. Hiervoor wordt de toekomstgerichte, observationele studie 
Early Developmental Stages of Psychopathology (EDSP) voorgesteld die een grote, algemene steekproef van 3021 adolescenten en jonge volwassenen van München, Duitsland, bevat, die gedurende 10 jaar werden gevolgd.

Hoofdstuk 2 onderzoekt het verband tussen psychotische psychopathologie en de het intermediaire fenotype van de neurocognitie in niet-affectieve psychose, aangezien het aantonen van differentiële patronen van cognitieve prestatie onder de verschillende psychopathologische dimensies inzicht zou kunnen bieden in de onderliggende mechanismen die resulteren in de alomtegenwoordige heterogeniteit inherent aan het psychosefenotype. Een systematische studie van de gepubliceerde studies die de correlaties tussen symptoomdimensies en de neuronpsychologische prestatie onderzoeken, werd uitgevoerd - de samenvattingen van 3129 artikels werden onderzocht om mogelijk in de analyse te worden opgenomen. Onze metaanalyse (58 studies, 5009 individuen) toonde aan dat negatieve en verstoorde dimensies duidelijk maar in bescheiden mate geassocieerd zijn met cognitieve gebreken, terwijl dit bij affectieve en positieve dimensies niet het geval was. De associatiepatronen van de vier psychosedimensies waren stabiel over de neurocognitieve domeinen heen en stonden los van leeftijd, geslacht en chroniciteit van de ziekte. Daarom besloten we dat psychotische symptomen en neurocognitie niet volledig orthogonaal zijn, wat suggereert dat de differentiële hersenmechanismen de oorzaak kunnen zijn van verstoorde en negatieve versus positieve en affectieve symptoomdimensies.

Hoofdstuk 3 beschrijft gegevens van de grote EDSP-steekproef van adolescenten en jonge volwassenen in de algemene populatie. Voor zover wij weten is dit de eerste studie die de invloed van de persistentie van subklinische psychotische ervaringen onderzoekt in relatie tot de transitie naar klinisch relevante psychose. De hypothese werd onderzocht dat ontwikkelende expressie van psychometrisch risico, in de vorm van subklinische psychotische ervaringen in de algemene populatie gewoonlijk van voorbij gaande aard is maar in sommige gevallen abnormaal kan aanhouden en kan evolueren tot een klinische, psychotische status. De bevindingen zijn i), subklinische psychose werd bevestigd als zijnde algemeen voorkomend en meestal tijdelijk, ii) aanhoudende subklinische positieve op psychose lijkende symptomen zijn een aanduiding van een groeiende klinisch relevante psychopathologie. Er werd een dosisreactiegradiënt aangetoond: hoe langer de subklinische psychose duurt, hoe groter het risico op ontwikkeling van een klinische stoornis, iii) hoewel in de meeste gevallen de subklinische psychose tijdelijk was, kon men het verband tussen de klinische en subklinische manifestaties niet verwaarlozen: een duidelijke proportie van de psychotische stoornissen werden het best geformuleerd als het magere resultaat van een relatief gewoon ontwikkelingsfenotype gekenmerkt door het aanhouden van psychometrisch opspoorbare subklinische psychotische ervaringen. Voor sommige individuen is de 
psychose bijgevolg het resultaat van een lang ontwikkelingsproces van subklinische ervaringen en functionele gebreken. Dit kunnen we samenvattend beschrijven als een model van psychotische stoornis op basis van psychosevatbaarheid en aanhoudende beschadiging. Mogelijke mechanismen die de overgang van aanhoudende subklinische psychose naar beschadiging verklaren zijn wellicht gebaseerd op gedrags-, omgevings- en neurobiologische interacties.

Hoofdstuk 4 beschrijft gegevens van de EDSP-studie die realiteitsvertekening en affectieve dysregulatie verbindt vanuit een risicoperspectief, op een op populatie gebaseerd niveau. Het onderzoekt het verband tussen afgezwakte positieve en affectieve symptomen en het begin van een klinische psychose. De resultaten suggereren dat psychotische ervaringen in de algemene populatie verband houden met affectieve dysregulatie en leveren een bewijs van het bestaan van een dosisreactiegradiënt. Bovendien zijn het aanhouden van op psychose gelijkende ervaringen en het begin van de beschadiging sterker wanneer een affectieve dysregulatie aanwezig is. In het licht van het groeiende bewijs dat een overlapping suggereert in genetische vatbaarheid over de verschillende psychosecategorieën heen, suggereren onze bevindingen dat genetische vatbaarheden die onderliggend zijn aan affectieve en niet-affectieve psychotische syndromen kunnen worden uitgedrukt als gecorreleerde dimensies in de algemene populatie. De langetermijnvoorspelling van realiteitsvertekening in affectieve dysregulatie wordt besproken in de context van bestaande psychologische modellen van symptoomvorming en neurobiologische activiteit, wat suggereert dat een proces van foutieve toekenning van emotionele saliëntie aan de bron ligt van dit verband.

Hoofdstuk 5 beschrijft gegevens van de EDSP-studie die het natuurlijke voorkomen van negatieve symptomen in de algemene populatie onderzoekt en of en hoe verzwakte positieve en negatieve maten van psychopathologie, vanuit een risicoperspectief, kunnen bijdragen tot het ontstaan van klinische psychose. A priori hypotheses suggereren dat cognitieve / negatieve symptomen die zijn geassocieerd met schizofrenie veranderingen in de hersenontwikkeling kunnen vertonen die verband houden met gedistribueerd genetisch risico. Wanneer het wordt gecombineerd met stressgerelateerde blootstellingen aan de omgeving kan een "final common pathway" (een uiteindelijk in dezelfde richting wijzend pad) van de neurotransmitterdysregulatie leiden tot het ontstaan van positieve psychotische symptomen. Deze studie benadrukte het natuurlijke voorkomen van verzwakte negatieve symptomen bij verder gezonde individuen, los van antipsychotische behandeling. Negatieve symptomen onthulden een patroon van sociodemografische verbanden die indicatief zijn voor ontwikkelingsbeschadiging, terwijl de positieve symptomen in verband werden gebracht met omgevingsrisicofactoren zoals trauma, cannabisgebruik en verstedelijking. Beide psychosedimensies kwamen frequent voor en kwamen vaker in combinatie voor dan bij willekeur kon worden voorspeld. 
Negatieve symptomen voorspelden mettertijd positieve symptomen, en het tegelijk voorkomen van positieve en negatieve symptomen was een aanwijzing voor klinische relevantie voor wat betreft secundaire functionele beschadiging en hulpzoekend gedrag. Resultaten suggereerden dat de vatbaarheden die onderliggend zijn aan negatieve kenmerken van psychotische ziekte worden verspreid op het niveau van de populatie en de ontogenese van positieve psychotische ervaringen voeden na blootstelling aan omgevingsrisico's, wat de waarschijnlijkheid op beschadiging en de zorgbehoefte vergroot.

In Hoofdstuk 6 worden de resultaten van deze thesis, met inbegrip van de systematische studie van klinische op de populatie gebaseerde studies, besproken en geïntegreerd in Een dynamisch model van het begin van klinische psychose vanuit epidemiologisch perspectief. Hier worden ook de nosologische en klinische implicaties van de bevindingen van deze thesis vermeld, alsook richtingen voor toekomstig onderzoek. 


\section{ACKNOWLEDGEMENTS}

To everyone who contributed towards the realization of this thesis, I thank you for sharing this exhilarating and enriching experience.

Many thanks to my promotor, Jim van Os, for guiding me along this research path with such geniality. Thank you for sharing your creativity, analytical understanding and broad knowledge-base of human mentation and psychosis. To my copromotors, Marieke Wichers, for your care during my time in Maastricht, and your support during my first STATA statistical analyses -thanks. Dear Lydia Krabbendam, the quality of your research is an inspiration. For your support and encouragement during my $\mathrm{PhD}$, I am truly grateful.

To all the collaborators I have met during my time here in Maastricht a big thank you. Wolfgang Viechtbauer, for your overwhelming meta-analytical knowledge. Claudia Simons, for your contribution in the neurocognitive data extraction task. Meram Can Saka, what an opportunity to have met in this country, far away from home which has resulted in this research collaboration. Inge van Rossum, for disentangling the affective dysregulation in the psychotic world. Deniz Arik, for sharing my introduction to social interaction studies, through our joint clinical perspective. Miriam Dietvorst and David, for all the Dutch translation and sharing your office space during my very first year. Nicole Geschwind, for the two years in which we have kindly shared every single day of research. Anne Fett, for the agreeable times working together. Mari Aguilera, my Spanish namesake, for our friendship kindled during our "dummiest" first research steps in this Dutch-land. Judith Allardyce, for your thoughtful psychiatric discussions, your friendship and, last but not least, thanks for having gone through all the writing of the current thesis!

To the research group of the Early Developmental Stages of Psychopathology Study at the Max Planck Institute of Psychiatry, Munich, Germany; Prof. dr. Uli-Hans Wittchen and Prof. dr. Roselind Lieb, Thank you for this special opportunity to collaborate and analyse this unique dataset. Thank-you for your useful comments and unhesitatingly support during the publication process. 
Finally, I would like to thank to all the colleagues of the Department of Psychiatry and Neuropsychology at Maastricht University.

My journey started in Spain, I need to thank Ana González Pinto for introducing me to psychiatric research, in Vitoria, Spain, whose fruitful research collaborations allowed me to work with Jim van Os in Maastricht. I would like to thank all my clinical psychiatrist colleagues and mentors at the Santiago Apostol Hospital, in Vitoria. Especially: Maite Ramírez, for introducing me in the interesting world of the psychopathological evaluation; Ana Blanca Yoller, for your encouraging and keen psychiatric rehabilitation to the people suffering from severe mental disorder; Sofía Álvarez de Eulate, for initiating me in the psychotherapeutic approach; Juan Medrano, for being as a psychiatric opened book and thank you for your truthful mentorship; and undoubtedly Ainara Jiménez, for who the appealing world of psychiatry provided the base for our psychiatric and 'real life' friendship.

To my nearest and dearest, whose support, love and friendship have been immeasurable. Ana and Pilar, my heartfelt thanks, for sharing our lives in this intense lived experience. Berta for our deep and never-ending friendship and your uncountable flights! Mia and Jamil, thanks for your proximity, and sharing your culture so warmly here in Maastricht. Claudia Vaz, Maastricht will always be the starting point of our friendship. My family, papa, mama, Javier, Nati, Pablo y Javier, Segundo y Raquel, thanks for giving me not only life, but also for giving me a enriching perspective on life. It is with deep gratitude that I thank all those people who see, trust and decide to walk through the treasures of life, with the truth in their hearts, and the depth and freedom of their love. Desde lo más profundo de mi ser, mi corazón agradecido. 


\section{CURRICULUM VITAE}

María de Gracia Domínguez was born on November, 1974 in Pamplona, Spain. She attended medical school at the University of Navarra, Spain. She completed her specialist clinical training in psychiatry at the Santiago Apostol Hospital in Vitoria, Spain (2002-2006). Her interest in academic psychiatry developed when she was a clinical trainee and began her academic career in the Department of Neuroscience, Santiago Apóstol Hospital, University of the Basque Country, UPV-EHU, Spain. She completed the doctorate courses in neuroscience (2003-2004), obtaining the certificate of Advance Studies in Neuroscience (2005). In 2006 she received a Marie Curie Early Stage Research Training Fellowship, and moved to the Research Institute for Brain and Behaviour, Department of Psychiatry and Neuropsychology, Maastricht University, The Netherlands, headed by Prof. Dr. Jim van Os, where she has developed her interest and expertise in psychiatric epidemiology. She attended epidemiology courses at Harvard School of Public Health, Harvard University, Boston MA, USA, while studying towards a $\mathrm{PhD}$ and continues to work in the Department of Psychiatry \& Neuropsychology, School of Mental Health and Neuroscience, Maastricht University. 



\section{PUBLICATIONS}

\section{Publications}

Van Rossum, Inge; Dominguez, Maria de Gracia; Lieb, Roselind; Wittchen, Hans-Uli; Van Os, Jim. Affective Dysregulation and Reality Distortion: A Longitudinal Investigation of their Association and Clinical Relevance. Schizophrenia Bulletin, doi:10.1093/schbul/sbp101

Dominguez, Maria de Gracia; Wichers, Marieke; Lieb, Roselind; Wittchen, HansUli; Van Os, Jim. Evidence that Onset of Clinical Psychosis is the Outcome of Progressively more Persistent Subclinical Psychotic Experiences: an 8-year Cohort Study. Schizophrenia Bulletin doi:10.1093/schbul/sbp022

Dominguez, Maria de Gracia; Viechtbauer, Wolfgang; Simons, Claudia J.P.; Van Os, Jim; Krabbendam, Lydia. Are Psychotic Psychopathology and Neurocognition Orthogonal? A Systematic Review of their Associations. Psychological Bulletin, 2009.Vol.135, Iss.1. pages 157-171

\section{Submitted papers}

Dominguez, Maria de Gracia; Can Saka, Meram; Lieb, Roselind; Wittchen, HansUli; Van Os, Jim. Onset of Psychotic Illness: Negative Symptoms Increasing Risk for Positive Symptoms Increasing Risk for Impairment? A 10-year Cohort Study. Submitted, under second revision Am J Psychiatry

Van der Werf, Margriet; Thewissen, Viviane; Dominguez, Maria de Gracia; Lieb, Roselind; Wittchen, Hans-Uli; Van Os, Jim. Adolescent Development of Social Reasoning Skills and Psychosis: The Case of Hearing Impairment. Submitted

Fett, Anne; Dominguez, Maria de Gracia; van Os, Jim; Penn, David; Krabbemdam, Lydia. Social and Neurocognition and their Association with Functional Outcome: A Systematic Review. Submitted

Dominguez, Maria de Gracia; Arik-Binbay, Deniz; Krabbemdam, Lydia; van Os, Jim. Psychotic Psychopathology and Social Cognition: A Meta-analysis of their Associations. Submitted 


\section{Book chapters}

Rutten Bart P.F., van Os Jim, Dominguez Maria de Gracia, Krabbendam, Lydia. Epidemiology and Social Factors: Findings from the Netherlands Mental Health Survey and Incidence study (NEMESIS). In: Persecutory delusions. Assessment, theory and treatment. Freeman, D., Bentall R., Garety P. Oxford University Press, 2008

Dominguez Barrera, Maria de Gracia; Ruiz Ibáñez, Iván; Valcárcel Huerta, César. Differential diagnosis in Bipolar Disorder. In: Educational Plan on Bipolar Disorder. M-29038-2006 Ed. Elsevier Farma, October, 2006.

Jiménez Ainara, Domínguez Maria de Gracia, Enjuto Sonsoles, Elorza Juan, Micó Jose Antonio, González-Pinto Ana. Neurocognition in Bipolar Disorder: Psychopharmacology. In: Investigation and Clinical Practice in Psychiatry. Pichot P, Ezcurra J, González Pinto A, Gutiérrez M (eds) Ed. Aula Médica Madrid, 2004.

\section{Oral communications}

Dominguez, Maria de Gracia; Viechtbauer, Wolfgang; Simons, Claudia J.P.; Van Os, Jim; Krabbendam, Lydia. Are Psychotic Psychopathology and Neurocognition Orthogonal? A Ssystematic Review of their Associations. International Congress on Schizophrenia Research, March 28 - April 01, 2009, San Diego, USA

Van Os, Jim; Dominguez, Maria de Gracia; Wichers, Marieke; Lieb, Roselind; Wittchen, Hans-Uli. "Evidence that Onset of Clinical Psychosis is the Outcome of Progressively more Persistent Subclinical Psychotic Experiences: Implications for Early Intervention”. International Congress on Schizophrenia Research, March 28 - April 01, 2009, San Diego, USA

Dominguez, Maria de Gracia. Psychosis in Community Mental Health. Opening conference of the Expert Course for Professional Development on Community Mental Health. Invited Conference. 11 November, 2008. Vitoria, Spain.

Dominguez, Maria de Gracia; Wichers, Marieke; Lieb, Roselind; Wittchen, HansUli; Van Os, Jim. Evidence that Onset of Clinical Psychosis is the Outcome of Progressively more Persistent Subclinical Psychotic Experiences: an 8-year Cohort. Study. 1st Schizophrenia International Research Society Conference. 21-24 June, 2008. Venice, Italy.

Dominguez, Maria de Gracia; Wichers, Marieke; Lieb, Roselind; Wittchen, HansUli; Van Os, Jim. Evidence that Onset of Clinical Psychosis is the Outcome of Progressively more Persistent Subclinical Psychotic Experiences: an 8-year Cohort Study. 14th Association of European Psychiatrist (AEP) Section for Epidemiology and Social Psychiatry, 11-14 June, 2008. Duvrovnik, Croatia. 
Dominguez, Maria de Gracia. The Genetic and Environmental Determinants of Persistence of Developmental Expression of Psychosis: Associations among Psychopathology and Neurocognition in Psychosis. Marie-Curie Fellow Meeting, February $22^{\text {th }}, 2008$. Maastricht, The Netherlands.

Dominguez, Maria de Gracia. The Genetic and Environmental Determinants of Persistence of Developmental Expression of Psychosis: Psychotic Disorder, a Rare Phenomenon within a Common Phenotype? Marie-Curie Fellow Meeting, February $28^{\text {th }}, 2007$. Maastricht, The Netherlands.

Jiménez Ainara; Dominguez, Maria de Gracia. Neuropsychological Impairment in First Onset of Bipolar Disorder. II National Symposium: The Earliest Phases of Mental Disorders: The Bipolar Spectrum. November $25^{\text {th }}-27^{\text {th }}, 2004$. Santander, Spain.

Dominguez, Maria de Gracia. Psychiatric Diagnosis and Imputation. XIII National Congress of Legal Psychiatry. October $6^{\text {th }}-8^{\text {th }}, 2004$. Málaga. Spain.

Dominguez, Maria de Gracia; Jimenez, Ainara. Neurocognition in Bipolar Disorder -Euthimic Patients- and Psychopharmacology. Symposium: Neuroscience, Psychopathology and Bipolar Disorder. July 2th, 2004. Vitoria. Spain.

Dominguez, Maria de Gracia; Jimenez, Ainara. Neurocognition in psychosis. Symposium: Cognition and Mental Disorders. May $18^{\text {th }}, 2004$. Fuenterrabia. Spain.

\section{Posters}

Dominguez, Maria de Gracia; Viechtbauer, Wolfgang; Simons, Claudia J.P.; Van Os, Jim; Krabbemdam, Lydia. Associations among Psychopathology and Neurocognition in non-Affective Psychosis. Abstract book. Research Day's School of Mental Health and Neuroscience. Maastricht. March, 2009.

Dominguez, Maria de Gracia; Viechtbauer, Wolfgang; Simons, Claudia J.P.; Van Os, Jim; Krabbemdam, Lydia. Are psychotic psychopathology and neurocognition orthogonal? A systematic review of their associations. Schizophrenia Research. Abstracts. Vol.102/1-3, Supplement 2, June 2008. pg. 118

Dominguez, Maria de Gracia; Viechtbauer, Wolfgang; Simons, Claudia J.P.; Van Os, Jim; Krabbemdam, Lydia. Are psychotic psychopathology and neurocognition orthogonal? A meta-analysis. Abstract book. $14^{\text {th }}$ AEP Symposium, 11-14 June 2008 / Duvrovnik - Croatia. P-26 section. Pg 96.

Barbeito, Sara; Álvarez de Eulate, Sofía; Ron, Santiago; Ramírez, Fernando; Mosquera, Fernando; Villamor, Ana; Domínguez, Maria de Gracia; García, Manuel; Aspiazu, Sofia; González-Pinto, Ana. Suicide prevention in early psychosis”. IX National Psychiatric Congress. Pamplona. Spain, October 2005. 
Aldama, Ana; González, Cristina; González-Pinto, Ana; Mosquera, Fernando; Ramírez Fernando; Domínguez, Maria de Gracia. Searching for Affective Symptoms as Risk Factors for Suicide in Early Psychosis. 18th ECNP Congress. Amsterdam. Netherlands. October 2005.

Martínez, Pilar; Jiménez, Ainara; Domínguez, Maria de Gracia; Figuerido, Juan Luis; Tapia, Eider; González-Pinto, Ana. Antipsychotic Treatment in Bipolar Patients whit Manifest Sggressiveness. VII National Psychiatric Congress. Bilbao. Spain. October 2004.

Jiménez, Ainara; Tapia, Eider; Domínguez, Maria de Gracia. Working Conditions and Legal Involvement of Internal Physician Residents in Psychiatry. XIII National Congress of Legal Psychiatry. Málaga. Spain. October 2004.

Imaz, Harkaiz; López, Purificación; Ramírez, Maite; Jiménez, Ainara; Domínguez, Maria de Gracia; Micó, Juan Antonio; González-Pinto, Ana. Treatment with Olanzapine in Severe Bipolar Patients I with Quickly Cycles during Dysphoric Mania. VII National Psychiatric Congress. Palma de Mallorca. Spain. October 2003.

Alonso, Marta; Ramírez, Fernando; Domínguez, Maria de Gracia; Imaz, Harkaitz; González-Oliveros, Ruben; Figuerido, Juan Luis; González-Pinto, Maria Asunción; González, Cristina; Montalbán, Raul; González-Pinto, Ana. Depression Treatment in Severe Bipolar Patients: Decrease in Switching. VII National Psychiatric Congress. Palma de Mallorca. Spain. October 2003.

\section{Research grants}

Marie Curie Early Stage Research Training Fellowship of the European Community's Sixth Framework Program under contract number "MEST-CT2005-020589" (July 2006 - June 2008). 